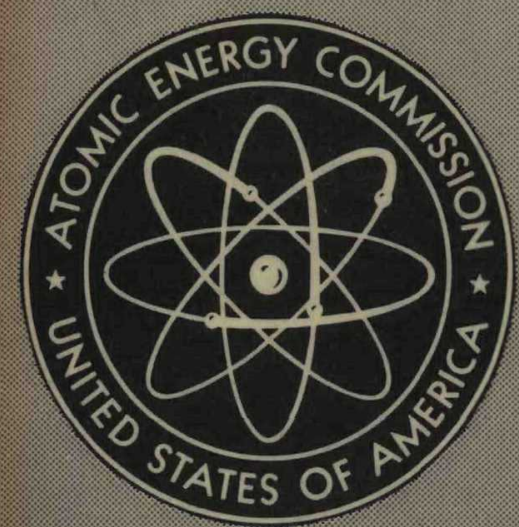

\title{
GA-1038
}

CHEMISTRY-GENERAL

\section{THERMODYNAMIC AND TRANSPORT PROPERTIES OF GASEOUS CARBON DIOXIDE}

By

L. H. Chen

August 10, 1959

Electric Boat Division

General Dynamics Corporation

Groton, Connecticut 


\section{DISCLAIMER}

Portions of this document may be illegible in electronic image products. Images are produced from the best available original document. 


\section{LEGAL NOTICE}

This report was prepared as an account of Government sponsored work. Neither the United States, nor the Commission, nor any person acting on behalf of the Commission:

A. Makes any warranty or representation, expressed or implied, with respect to the accuracy, completeness, or usefulness of the information contained in this report, or that the use of any information, apparatus, method, or process disclosed in this report may not infringe privately owned rights; or

B. Assumes any liabilities with respect to the use of, or for damages resulting from the use of any information, apparatus, method, or process disclosed in this report.

As used in the above, "person acting on behalf of the Commission" includes any employee or contractor of the Commission, or employee of such contractor, to the extent that such employee or contractor of the Commission, or employee of such contractor prepares, disseminates, or provides access to, any information pursuant to his employment or contract with the Commission, or his employment with such contractor.

\section{This report has been reproduced directly from the best available copy.}

Printed in USA. Price \$2.75. Available from the Office of Technical Services, Department of Commerce, Washington 25, D. C. 
GA- 1038

THERMODYNAMIC AND TRANSPORT PROPERTIES OF GASEOUS CARBON DIOXIDE

by

L. H. Chen

August 10, 1959

\section{GENERAL DYNAMICS \\ $\begin{array}{lllllllllll}C & O & R & P & O & R & A & T & I & 0 & N\end{array}$}

ELECTRIC BOAT DIVISION

Contract AT(04-3)-187 with the

U. S. Atomic Energy Commission

and the Maritime Administration 


\section{Table of Contents}

Page

Acknowledgements 1

I Introduction 3

II Symbols and Units 7

III Survey of Existing Data 9

A. Pressure-Volume-Temperature Relations 9

B. Specific Heats 13

C. The Joule-Thomson Expansion 15

D. Viscosity 15

E. Heat Conductivity 17

F. Velocity of Sound 18

IV Calculation of Thermodynamic Functions and Construction 21 of the Diegrams

A. The Beattie-Bridgeman Equation of State 21

B. Computation of Entropy, Internal Energy Function, 22 Enthalpy, and Compressibility Factor

C. Construction of the Diagrams 25

D. Independent Checks by Joule-Thomson Data and 26 Michels' Isotherm

V Calculation of Constant-Volume Specific Heat, ConstantPressure Specific Heat, Specific-Heat Ratio, and the Velocity of Sound

A. The Basic Equations 27

B. Discussion $\quad 32$

VI Calculation of Absolute Viscosity, Heat Conductivity, 35 and Prandtl Number

A. The Basic Equations 35

B. Discussion $\quad 37$

VII Construction of the T-s and the h-s Diagrams in the 41 Saturated Region 
IX Figures 49

Fig. I Comparison of Compressibility Data

Fig. 2 Comparison of $\mathrm{Cp}$ at 1 atm

Fig. 3 Comparison of $\mathrm{Cp}$ at $10 \mathrm{~atm}$

Fig. 4 Comparison of $\mathrm{Cp}$ at $40 \mathrm{~atm}$

Fig. 5 Comparison of $\mathrm{cp}$ at $100 \mathrm{~atm}$

Fig. 6 Comparison of $\mathrm{cp}$ at $150 \mathrm{~atm}$

Fig. 7 Comparison of $\mathrm{Cp}$ at 200 atm

Fig. 8 The Variation of $\mathrm{Cv}_{\infty}$ with Temperature

Fig. 9 Reduced-Scale Temperature-Entropy Diagram

$3 \mathrm{~J}$ ig. Il Reduced-Scale Enthalpy-Entropy Diagram

6 Fig. 12 Reduced-Scale Chart for Compressibility Factor

7 Fig. 13 Reduced-Scale Chart for Specific Heat at Constant Pressure

4.Fig. 14 Reduced-Scale Chart for Specific Heat at Constant Volume

TFig. 15 Reduced-scale Chart for Specific Heat Ratio

1) Fig. 16 Reduced-Scale Chart for Velocity of Sound

12 Fig. 17 Reduced-Scale Chart for Viscosity

Fig. 18 Reduced-Scale Chart for Heat Conductivity

13Fig. 19 Reduced-Scale Chart for Prandtl Number

4 Fig. 20 Reduced-Scale Enthalpy-Entropy Diagram with Constant Compressibility Lines

$5 \sqrt{ }$ Fig. 21 Reduced-Scale Enthalpy-Entropy Diagram in the Saturated Region

2 Fig. 22 Reduced-Scale Temperature-Entropy Diagram in the Saturated Region

Table 1 Viscosity Data Taken for Correlation

Table 2 Comparison of Viscosity Data

Table 3 Comparison of Heat Conductivity Data

Table 4 Thermodynamic Properties of Gaseous 


\section{ACKNOWLEDGEMENTS}

The work described in this report was orlginally part of a subcontract to the Atomic Energy Commission through the General Atomic Division of General Dynamics Corporation. A preliminary report was issued in July 1957. The work of putting all the pertinent information in this final form was accomplished under the Marine Gas Cooled Reactor Program (MCCR). The cooperation of Messrs. A. C. McClure, D. F. Putnam, and J. M. Hinchey of the MGCR project is acknowledged.

The successful completion, in a relatively short time, of the voluminous computational work required was made possible by using an IBM 704 digital computer. The work of Dr. A. J. van Woerkom, Assistant Head of the Digital Computer Division, and Messrs. R. Spong and R. Elder is also acknowledged.

The work was accomplished under the direction of Dr. L. H. Chen, head of the Applied Mechanics Section. He was ably assisted by Mrs. V. D. Christian, Mr. M. P. Wilson, Jr., and others of the Applied Mechanics Section. The work was carried out under the overall direction of Mr. M.G.J. Boissevain, Chief Technical Services Engineer, and Dr. H.E. Sheets, Chief Research and Development Engineer.

The investigation and subsequent use of the Beattie-Bridgeman equation of state was originally suggested by Mr. E. S. Dennison, MGCR project director: His technical advice and constant interest in this work are gratefully acknowledged. Valuable suggestions regarding the 
correlation and calculation of transport properties were made by Professor F. G. Keyes of the Massachusetts Institute of Technology. They are acknowledged elsewhere in the report. 
INTRODUCTION

The purpose of this report is to present, under one cover, the thermodynamic and transport properties of gaseous carbon dioxide. These properties have been computed from the most recent data, and are presented here for ready reference. This information was urgently needed in connection with the application of carbon dioxide as a possible working medium for the closed-cycle gas turbine nuclear powerplant.

The most extensive work to date on the compilation of the thermodynamic data of carbon dioxide, together with a large scale temperature-entropy diagram in the range of interest to power cycle applications, is that of Sweigert, Weber, and Allen (Ref. 1). However, more recent data have indicated considerable discrepancies, particularly in the region of high pressure and low temperature. Therefore, it has become necessary to investigate these discrepancies in order to obtain a more reliable and consistent source. As for the transport properties, dependable values of viscosity and heat conductivity available in the published literature cover an entirely inadequate range. In particular, experimental data in the high temperature and high pressure range are lacking. However, most recent data and the method of density correlation for the pressure effect are available through the courtesy of Professor F.G. Keyes and his co-workers at M.I.T (Ref. 2 and 3 ). An extensive check with other available data has been made, and the 
results in this report are believed to be the most reliable and accurate as of this writing.

The computations in this report were performed by an IBM high speed digital computer. The results are presented in graphical form with pressure and temperature as independent variables, which are convenient for engineering calculations. A total of thirteen charts were constructed, namely:

1) Temperature-Entropy Diagram

2) Enthalpy-Entropy Diagram

3) Compressibility Factor

4) Specific heat at Constant Pressure

5) Specific Heat at Constant Volume

6) Specific Heat Ratio

7) Velocity of Sound

8) Absolute Viscosity

9) Heat Conductivity

10) Prandt I Number

11) Enthalpy-Entropy Diagram with Constant Compressibility Lines

12) Enthalpy-Entropy Diagram in the Saturated Region

13) Temperature-Entropy Diagram in the Saturated Region

These charts, with the exceptions of (12) and (13), cover a range of temperatures from $0 \mathrm{~F}$ to $1500 \mathrm{~F}$ and of pressures up to 4000 psia. The lower limit of specific volumes is down to $0.05 \mathrm{ft}^{3} / 1 \mathrm{~b}$. This range practically encompasses all possible conditions of interest in power cycle operations. The forms of these charts are shown in reduced scale graphs, which are included at the end of this report for 
illustration purposes (Fig. 10-22). They indicate a few of the lines actually drawn on the full scale charts and are not recommended for use in design calculations. Full scale charts are available upon request. sue 315 ia bach of mester. gou

Charts (12) and (13) cover a range of temperatures from $400 \mathrm{~F}$ down to $-50 \mathrm{~F}$. The portion of the charts from $400 \mathrm{~F}$ to the saturation curve is a duplication of charts (1) and (2) and is included for convenience in application. The saturation region is of possible interest in compressor design, since operating conditions of the compressor in a $\mathrm{CO}_{2}$ gas turbine plant could be close to the saturation region.

Tabulated values of specific volume, enthalpy, and entropy are included at the end of this report, Table 4. These values were printed directly from an IBM digital computer in intervals which permit an accuracy within $1 \%$ for linear interpolations. 
- 


\section{II}

SYMBOLS AND UNITS

Unless indicated otherwise in the text, the standard symbols and units used in this report are as follows.

\begin{tabular}{|c|c|}
\hline$p$ & pressure (psia) \\
\hline$t$ & temperature $\left({ }^{\circ} \mathrm{F}\right)$ \\
\hline $\mathbf{T}$ & absolute temperature $\left({ }^{\circ} R\right)$ \\
\hline $\mathbf{v}$ & specific volume $\left(\mathrm{ft}^{3} / \mathrm{lb}\right)$ \\
\hline s & specific entropy $\left(B t u / I b{ }^{\circ} R\right)$ \\
\hline $\mathrm{u}$ & internal energy function (Btu/lb) \\
\hline $\mathrm{h}$ & enthalpy (Btu/lb) \\
\hline $\mathbf{z}$ & compressibility factor \\
\hline $\mathrm{Cp}$ & constant-pressure specific heat $\left(B t u / I b{ }^{\circ} F\right)$ \\
\hline $\mathrm{Cv}$ & constant-volume specific heat $\left(\mathrm{Btu} / \mathrm{Ib} \mathrm{O}_{\mathrm{F}}\right)$ \\
\hline $\mathrm{Cv}_{\infty}$ & $\begin{array}{l}\text { constant-volume specific heat at zero pressure or } \\
\text { infinite volume }\left(\mathrm{Btu} / \mathrm{lb}^{\circ} \mathrm{F}\right)\end{array}$ \\
\hline$\gamma$ & ratio of $\mathrm{Cp}$ to $\mathrm{Cv}$ \\
\hline M & molecular weight $=44.011$ \\
\hline g & gravitational acceleration $=32.17 \mathrm{ft} / \mathrm{sec}^{2}$ \\
\hline$\rho$ & density $\left(1 \mathrm{~b} / \mathrm{ft}^{3}\right)$ \\
\hline Vs & velocity of sound (fps) \\
\hline K & heat conductivity $\left(\mathrm{Btu} / \mathrm{hr}{ }^{\mathrm{O}_{\mathrm{F}} \mathrm{ft}} \mathrm{f}\right)$ \\
\hline$\mu$ & absolute viscosity (lb/hr ft) \\
\hline $\operatorname{Pr}$ & Prandtl number $\left(\frac{\mathrm{C}_{\mathrm{P}} \mu}{\mathrm{K}}\right)$ \\
\hline
\end{tabular}


$\mid$

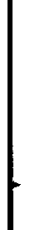

-

$-$

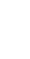




\section{A. Pressure - Volume - Temperature Relations}

In principle, all. the thermodynamic functions of a fluid can be evaluated from pressure, volume and temperature relations ( p-v-t data), and some supplementary data. These supplenentary data may be the variation of $\mathrm{C}_{\mathrm{p}}$ with temperature along any one line of constant pressure; the variation of $C_{V}$ with temperature along any one line of constant volune; or any measurenents that give values of $u, h, s$, or any of their derivatives, such as the Joule-Thomson coefficients. The development of spectroscopic methods of determining the specific heats at zero pressure has reduced the degree of uncertainty of this source of supplementary data for $\mathrm{CO}_{2}$ to such an extent that there is now little room for disagreement. Therefore, the degree of uncertainty in the calculation of thermodynamic functions of $\mathrm{CO}_{2}$ depends mainly upon the state of knowledge of $p-v-t$ relations or compressibility data.

Prior to 1935, the work of Michels and his co-workers in the Van der Waals Laboratory (Ref. 4 and 5) yielded the most accurate data, ranging from $32 \mathrm{~F}$ to $302 \mathrm{~F}$ and from about 300 psia up to 44,000 psia. A special technique was devised for measuring the isotherms near the critical region, and the accuracy of the data is of a high order. In 1950 MacCormack and Schneider (Ref. 6) reported accurate pv data up to $1112 \mathrm{~F}$ and 735 psia. Kennedy of Harvard obtained the most recent and extensive data (Ref, 7 and 8 ). These cover the range from $212 \mathrm{~F}$ 
to $1832 \mathrm{~F}$ and 735 psia to 20,600 psia. Kennedy's data agree well with Michels', MacCornack's, and Schneider's. It is therefore obvious that a combination of these data covers a sufficient temperature range for power cycle applications. The pressure range is indeed more than adequate.

Various equations of state and methods of data-fitting have been employed to correlate the experimental data. Plank and Kuprianoff (Ref. 9), and Sweigert, Weber, and Allen (Ref. 1) used a simple twoconstant equation of state to calculate the thermodynamic properties in the superheated vapor region. The work of Plank and Kuprianoff was the most extensive, in the period of 1929 to 1948, on the compilation of thernodynamic data of $\mathrm{CO}_{2}$ together with a temperatureentropy diagram. Their diagram, however, was prepared primarily for use in the refrigeration industry, and its temperature and pressure range for superheated vapor does not go beyond $300 \mathrm{~F}$ and 1700 psia. The work of Sweigert et al. was based on available experimental data prior to 1948 within the range up to $500 \mathrm{~F}$ and 3000 psia. It includes the accurate data of Michels and his co-workers. Sweigert's computed enthalpy values agree well with more recent data in the low pressure region. However, considerable discrepancies have been found in the high pressure and low temperature region. The Sweigert values are believed to be in error, probably because his two-constant equation of state is inadequate to cover a range in which the specific volume of the fluid is down to the neighborhood of 0.3 to $0.1 \mathrm{ft}^{3} / 1 \mathrm{~b}$. For closedcycle gas turbine applications, Sweigert's temperature-entropy diagram is inadequate for compressor performance calculations. 
Among the more important and most recent reports on the correlation and compilation of thermodynamic data are those of Hilsenrath et al. of the National Bureau of Standards (Ref. 10), Newitt et al. of the British Imperial College (Ref. 11), and Price of the Naval Ordnance Laboratory (Ref. 12). Hilsenrath et al. have correlated experimental data up to 1950, namely MacCormack's and Schneider's. The range covered was up to $2440 \mathrm{~F}$ and 1470 psia. Newitt's data, although published in 1956, was essentially based on Michels' data (1935) in the superheated vapor region. A temperature-entropy diagram was constructed which includes the liquid and solid phases. The temperature range is only up to $302 \mathrm{~F}$. Serious discrepancies of as much as $20 \%$ have been found in the enthalpy values of Hilsenrath and of Newitt, in the high pressure range ( $1470 \mathrm{psia}$, which is the maximum range covered by Hilsenrath). Since Michels' data have been used either directly or indirectly by both authors, it is obvious that the sources of discrepancy are the computation methods rather than the basic experimental data used.

Price's work has included the most recent $p-v-t$ data measured by Kennedy. The data were smoothed by a graphical method in which the compressibility factor was plotted against density to form a mesh of isobars and isotherms. Based on these smoothed data, numerical differentiation and integration were used for the computations of all the thermodynamic functions. Price made no attempt to find an equation of state to fit the smoothed data. The numerical method is justified in view of the fact that no single equation of state could possibly represent Kennedy's data, which cover a wide pressure range, such as from 735 psia to 20,600 psia. 
However, in closed-cycle gas turbine applications, it is adequate to limit the range of temperatures from $0 \mathrm{~F}$ to $1500 \mathrm{~F}$ and pressure up to 4000 psia with specific volume down to $0.05 \mathrm{ft}^{3} / 1 \mathrm{~b}$. Within this range it is found that the Beattie-Bridgeman equation (Ref. 13), published in 1927, is well confirmed by the most recent data. Michels (Ref. 14) compared the Beattie-Bridgeman equation with his experimental pv data and found that the equation fits the experiments within an accuracy of $0.7 \%$ up to 112 Amagat density (about $0.0718 \mathrm{ft}^{3} / 1 \mathrm{~b}$ in specific volume). Figure 1 compares Michels' and Kennedy's smoothed data with the Beattie-Bridgeman equation at a few isobars. Within the range of primary interest in power cycle applications, the BeattieBridgeman equation together with the spectroscopic data of $c_{p}$ at low pressure will provide a most accurate and consistent source of data from which the computation of all the thermodynamic functions of $\mathrm{CO}_{2}$ may be performed.

In a recent paper, Davy and Bell (Ref. 15) have evaluated a total of ten equations of state, including the Beattie-Bridgeman equation, for the representation of $\mathrm{CO}_{2}$ data (Ref. 4, 5, 6) at four 1sotherms. They have found that the Su-Chang equation of state (Ref. 16) gives in general a best fit of the available data for pressures up to a reduced pressure of 10 , including the region close to the liquid boundary. However, for the range of interest of our work (specific volumes greater than $.05 \mathrm{ft}^{3} / 1 \mathrm{~b}$ and reduced pressures less than 4), it may be seen from Davy's comparison that both the Su-Chang and the BeattieBridgeman equations give essentially identical results. It would be 
interesting for any future work to compute the thermodynamic properties of carbon dioxide from the Su-Chang equation of state for its wider range of validity.

\section{B. Specific Heats}

The specific heats of $\mathrm{CO}_{2}$ at constant pressure and constant volume have been the subjects of numerous investigations. Leduc (Ref. 17) made a critical survey of the available data prior to 1929. Quinn and Jones (Ref. 18) discussed these data and later work published through 1935. Hilsenrath (Ref. 10) and Newitt (Ref. 11) made brief surveys of the more recent data.

The specific heat of $\mathrm{CO}_{2}$ at low pressures rests on an assured basis. Experimental data measured by the calorimeter method and by the velocity-of-sound method agree well with the spectroscopic data, which are determined by the application of quantum theory. On the other hand, only fragmentary data on experimental specific heats are available for $\mathrm{CO}_{2}$ at pressures above 1 atmosphere.

An extensive comparison of $c_{p}$ values available in the more recent literature was made in our work. Some pertinent results are included in this report (Fig. 2-7). Among the more important work taken for comparison is that of Ellenwood et al. (Ref. 19), Hilsenrath et al. (Ref. 10), Newitt et al. (Ref. 11), and Price (Ref. 12). Ellenwood's work was based on the Beattie-Bridgeman equation of state together with the spectroscopic data of $C_{p}$ at zero pressure. Newitt's values were obtained by a strictly numerical method in accordance with the difference equation 


$$
c_{p}=\left(\frac{\Delta H}{\Delta T}\right)_{p}
$$

The enthalpy differences along the isobars were divided by $10 \mathrm{C}$ temperature intervals to give the $c_{p}$ values at the mean temperature of the intervals. It is obvious that these numerical procedures could lead to serious errors in the area close to the saturation curve. As is well known, the greatest dependence of $C_{p}$ on temperature is in this region. A small change in temperature will cause a very rapid change of $C_{p}$ as the value of $C_{p}$ becomes infinite at the critical point. The comparison of $\mathrm{C}_{\mathrm{p}}$ data indicates that all data agree well except those of Newitt. Significantly, at high pressures (for example, 200 atm in Fig. 7) Ellenwood's values agree with Price's most recent data on extrapolation to lower temperatures. This indicates that a combination of Ellenwood's and Price's data will provide a set of consistent $C_{p}$ values to cover a wide range of temperature and pressure conditions, namely $O \mathrm{~F}$ to $4000 \mathrm{~F}$ and 0 psia to 20,600 psia. For the purpose of our work, the use of the Beattie-Bridgeman equation of state within its range of validity is sufficient to obtain the desired $C_{p}$ values. Therefore, the computation of $C_{p}$ here is essentially a duplication of Ellenwood's effort. Since the $c_{p}$ values are needed to compute the velocity of sound as well as the Prandtl number, little extra effort is required to punch out its values from the IBM machine and to present them in a more accurate and complete graphical form than that given in page 13 of Ellenwood's paper (Ref. 19). 


\section{The Joule-Thomson Expansion}

The Joule-Thomson coefficient of $\mathrm{CO}_{2}$ was measured by several investigators prior to 1923. More recent measurements were made at the University of Wisconsin by Roebuck and his co-workers (Ref. 20) in their standard apparatus for measuring the integral Joule-Thonson effect. The Wisconsin measurements essentially supersede all the earlier ones since they are more precise and cover a large range (-58 F to $572 \mathrm{~F}$ and 14.7 psia to 2940 psia).

The experimental Joule-Thomson data are useful as an independent check of the computed thermodynamic functions. Roebuck's data were used in our report for checking purposes. The results will be discussed later.

\section{Viscosity}

Most of the published data on the viscosity and heat conductivity of $\mathrm{CO}_{2}$ are limited to low pressures. In a paper by Hawkins (Ref. 21), some 133 references prior to 1948 were examined for twelve gases; only one reference was given for viscosity and one for heat conductivity of $\mathrm{CO}_{2}$. The most extensive work published to date on the compilation of viscosity data of $\mathrm{CO}_{2}$ is Nuttall's (Ref. 22). The values of viscosity are tabulated for pressures of $1,10,40,70$, and 100 atmospheres with temperatures up to $2600 \mathrm{~F}$. Nuttall's viscosity values at $1 \mathrm{~atm}$ pressure were based mainly on the theoretical work of Hirschfelder et al. (Ref. 23 and 24 ). The values at higher pressures were computed from an empirical equation based on the experimental data of Phillips (Ref. 25); Stakelbeck (Ref. 26); and Comings, Mayland, and Egly (Ref. 27). 
Theoretical calculations of the viscosity of gases from kinetic theory have so far met with only limited success. The calculation of viscosity must make use of a molecular model for the gas, increased refinements being possible. Maxwell's classical theory is based on the simplest assumption of infinitely small, perfectly elastic molecules with zero fields of force. It predicts that the absolute viscosity of a gas is independent of pressure and that it increases in proportion to the square root of the absolute temperature. In 1948 Hirschfelder et al. published the tables of collision integrals based on the refined kinetic theory of Chapman and Cowling (Ref. 28), which permits accurate calculation of viscosity for nonpolar smooth spherical molecules. Eromley and Wilke (Ref. 29) have presented Hirschfelder's results in a convenient form for practical use. However, it may be noted that Hirschfelder's work is limited to the calculation of transport properties at low pressure only. In the case of carbon dioxide, the main difficulty lies in the complexity of the molecule, which has a permanent quadrupole and is not spherical. The fact that the viscosity of carbon dioxide fails to obey the Lennard-Jones 12:6 potential is noted, among others, by Whalley and Schneider (Ref. 30).

Experimental data on the effect of pressure upon viscosity of $\mathrm{CO}_{2}$, available in the published literature, cover an entirely inadequate range for closed-cycle gas turbine applications. The results of Phillips, Stakelbeck, Comings et al., and many others prior to 1950 were limited to low temperature conditions (up to about $300 \mathrm{~F}$ ). In 1952, Kiyama and Makita (Ref. 31) reported measurements covering a 
temperature range from $122 \mathrm{~F}$ to $572 \mathrm{~F}$ with pressure up to about 1400 psia. In the past decade, Keyes of M.I.T. has been active in correlating experimental data on the transport properties of 13 gases including $\mathrm{CO}_{2}$. Keyes has published his results in a series of ASME papers. His latest published results may be found in a technical report of Project Squid (Ref. 2). The method of using density instead of pressure for correlating pressure effect was made available through private communication with Professor Keyes. The correlation and computation of viscosity in our report was based on Keyes' method.

\section{E. Heat Conductivity}

The scarcity of heat conductivity data of $\mathrm{CO}_{2}$ is more serious than the lack of data for viscosity. Experimental data at high pressures are practically non-existent. Even for the data at 1 atm pressure, there is serious disagreement among the various investigators, and discrepancies in the order of 10 to $20 \%$ are not uncommon. However, this is not at all surprising in view of the experimental difficulties in eliminating heat transmission by convection ana radiation. Nuttall (Fef. 32,10 ) has complled heat conductivity data of $\mathrm{CO}_{2}$ at 1 atm pressure for the temperature range from $-136 \mathrm{~F}$ to $620 \mathrm{~F}$. A total of 13 references were listed in Nuttall's report. Among the more recent ones are Archer's (Ref. 33); Sherratt's and Griffiths' (Ref. 34); and Johnston's and Grilly's (Ref. 35).

At temperatures between $400 \mathrm{~F}$ to $600 \mathrm{~F}$, Archer's data disagree with Nuttall's tabulated values by as much as $11.8 \%$. 
Because of the lack of experimental data on the effect of pressure on heat conductivity, Gamson (Ref. 36) has employed a semi-theoretical approach to obtain a generalized correlation for heat conductivities of all gases. His results are presented in the familiar graphical form in terms of reduced quantities. Gamson's generalized attempt on heat conductivity is similar to Uyehara's and Watson's (Ref. 37) on viscosity. Gamson's method of correlation was essentially based on the considerations of Enskog's theoretical equations (Fef. 38), some experimental viscosity data, and the work of Uyehara and Watson. Gamson has estimated an accuracy of $15 \%$ for his results.

The computation of heat conductivity for $\mathrm{CO}_{2}$ in our work was based on Keyes' equations. Discussion of Keyes' equations and comparison with some Russian data are given in Section VI.

\section{F. Velocity of Sound}

Measurements of the velocity of sound in $\mathrm{CO}_{2}$ have been made by several investigators. The early work, prior to 1924, has been reviewed by Partington and Shilling (Ref. 39). King and Partington (Ref. 40), and Sherratt and Griffiths (Ref. 41) made measurements at 1 atm with temperature up to $1832 \mathrm{~F}$. Sherratt's and Griffiths' data were obtained at three frequencies $(7911.2,13828$, and $19365 \mathrm{cycles} / \mathrm{sec}$ ). In the case of $\mathrm{CO}_{2}$, it is well known that the velocity of sound is not independent of frequency. Sherratt and Griffiths made a correlation to reduce the observed velocities to correspond to zero frequency on the basis of a theory proposed by Kneser (Ref. 42). Kneser explained the variation of sound velocity with frequency in terms of the 
time lag in the transfer of energy between the vibrational and the other energy states of a molecule. However, between the two extreme frequency values used by Sherratt and Griffiths, the variation of sound velocity is less than $1 \%$, which is negligibly small from the standpoint of engineering application. The main purpose of Sherratt's and Griffiths' work was to obtain experimental data on sound velocity in $\mathrm{CO}_{2}$ so that the specific heats could be evaluated using the BeattieBridgeman equation of state. The specific heats thus obtained by Sherratt and Griffiths are in excellent agreement with those deduced from the spectroscopic data.

The effect of pressure on the velocity of sound in $\mathrm{CO}_{2}$ was investigated by Hubbard and Hodge (Ref. 43). They measured the sound velocity at $80.6 \mathrm{~F}$ with pressure up to about $880 \mathrm{psia}$. Anderson and Delsasso (Ref. 44), and Richardson (Fef. 45) have obtained experimental data in the region near the critical point $(87.8 \mathrm{~F}$ and 1070 psia). Above the critical temperature it was found that, as the pressure increases, the velocity decreases steadily, goes through a sharp minimum, and rises abruptly. Below the critical temperature a discontinuity in velocity was observed between liquid and vapor.

In theory, the velocity of sound at low frequencies may be computed from the equation of state. Hilsenrath et al. (Ref. 10) have compiled a tabulation of velocity values up to $2240 \mathrm{~F}$ and $1470 \mathrm{psia}$. In our work the Beattie-Bridgeman equation was used for computation. It was found that the computed results indicated the correct trend, but failed to agree in values with the experimental data of Anderson and 
Delsasso, and Hichardson in the region near the critical point. In this region the experimental data were therefore used instead of the computed values from the equation of state. A more detailed discussion is given in section $V$. It may be noted that the tabulated values of Hilsenrath et al. fail to represent even the correct trend as indicated by experimental evidences. 
CALCULATION OF THERMODYNAMIC FUNCTIONS AND

CONSTRUCTION OF THE DI AGRAMS

\section{A. The Beattie-Bridgeman Equation of State}

The Beattie-Bridgeman equation is given in the following form.

$$
P=\frac{R T(l-\epsilon)}{V^{2}}(V+B)-\frac{A}{V^{2}}
$$

where: $\quad A=A_{0}\left(I-\frac{a}{\nabla}\right)$

$$
\begin{aligned}
& B=B_{0}\left(1-\frac{b}{V}\right) \\
& \epsilon=\frac{c}{V^{3}}
\end{aligned}
$$

$P=$ absolute pressure $1 b / f t^{2}$

$T=$ absolute temperature ORankine

$\mathrm{V}=$ molal volume $\mathrm{ft} 3 / \mathrm{lb}$-mole

$R=1544 \mathrm{ft}-\mathrm{lb} /(\mathrm{lb}-\mathrm{mole})\left({ }^{\circ} \mathrm{R}\right)$

For carbon dioxide, the five characteristic constants consistent with the units given above are:

$$
\begin{aligned}
& A_{0}=2.71 \times 10^{6} \\
& a=1.140 \\
& B_{0}=1.676 \\
& b=1.158 \\
& c=6.16 \times 10^{7}
\end{aligned}
$$

Note that the Beattie-Bridgeman equation cannot be solved explicitly for $v$ as a function of $p$ and $T$. It is therefore necessary to choose $v$ and $T$ as independent variables in the evaluation and computation of 
thermodynamic properties. Cross-plottings are required in order to present the results, using $\mathrm{p}$ and $\mathrm{T}$ as independent variables which are convenient for engineering applications. The tabulated values of thermodynamic properties given in Table 4 of this report were obtained by Newton-Raphson's method of iteration.

The iteration process was performed with an IBM 704 digital computer, thus arriving at a correct value of $\mathrm{V}$ corresponding to a predetermined set of $p$ and $T$ values. The accuracy of the process was carried out to $0.01 \%$.

B. Computation of Entropy, Internal Energy Function, Enthalpy, and Compressibility Factor

One of the basic relations of thermodynamics, giving the change of entropy with $\mathrm{V}$ and $\mathrm{T}$ as independent variables, is

$$
T d s=C_{v} d T+T\left(\frac{\partial p}{\partial T}\right)_{v} \quad d v
$$

Integrating eq. (2) gives the difference in entropy between two states,

$$
\Delta S=\left[\int_{v_{1}}^{v_{2}}\left(\frac{\partial p}{\partial T}\right)_{v} d v\right]_{T}+\left[\int_{T_{1}}^{T_{2}} \frac{C_{v}}{T} d T\right]_{v}
$$

The integrand in the first term on the right-hand side of eq. (3) may be evaluated from the Beattie-Bridgeman equation (eq. 1).

$$
\left(\frac{\partial p}{\partial T}\right)_{v}=\frac{R}{v}+\frac{R B_{0}}{v^{2}}-\frac{R B_{o} b}{v^{3}}+\frac{2 c R}{v^{2} T^{3}}+\frac{2 c R B_{0}}{v^{3} T^{3}}-\frac{2 c R B_{0} b}{v^{4} T^{3}}-\cdot-\cdot-\cdot-\cdot--(4)
$$

The values of $\mathrm{C}_{\mathrm{v}}$ at infinite volume or zero pressure, $\mathrm{C}_{\mathrm{v}_{\infty}}$, may be found in reference 19. They were based on the spectroscopic data obtained by Johnston and his co-workers at Ohio State University. Figure 8 shows the variation of $C_{v_{\infty}}$ with temperature. A check of these 
values with the values computed from the equation given by Sweigert (Ref. 1) for $C_{\text {po }}$ through the perfect gas relation shows excellent agreement. Sweigert's equation was therefore adopted for evaluating the second term on the right-hand side of eq. (3).

Conforming to the commonly used engineering units as listed in Section II, the expression for entropy values as evaluated from eq. (3) and programmed in the digital computer was

$$
\begin{aligned}
s= & .04486\left\{\ln (44 \mathrm{v})-\frac{\mathrm{B}_{o}}{44 \mathrm{v}}\left(1-\frac{\mathrm{b}}{88 \mathrm{v})}\right) \frac{2 \mathrm{c}}{\left.\left.(44 \mathrm{v}) \mathrm{T}^{3}\left[1+\frac{\mathrm{B}_{o}}{88 \mathrm{v}}-\frac{\mathrm{B}_{\mathrm{ob}}}{3(44 \mathrm{v})}\right)^{2}\right]\right\}}\right. \\
& +\frac{1}{44}\left(14.2161 \ln \mathrm{T}+\frac{6.53 \times 10^{3}}{\mathrm{~T}}-\frac{0.705 \times 10^{6}}{\mathrm{~T}^{2}}\right)+s^{\prime \prime}-\ldots-(5)
\end{aligned}
$$

where $\mathbf{s}^{\prime \prime}$ is a constant of integration and is arbitrarily chosen as -1.099082 , so that the entropy value at $10 \mathrm{psia}$ and $1600 \mathrm{~F}$ is the same as Sweigert's (Ref. 1). The values of $B o, b$ and $c$ are the same as in eq. (Ia).

From the first and second laws of thermodynamics we may write

$$
d u=T d s-p d v
$$

Substituting in eq. (2)

$$
\begin{aligned}
d u & =C_{v} d T+T\left(\frac{\partial P}{\partial T}\right)_{v} d V-p d v \\
& =C_{v} d T+\left[T\left(\frac{\partial p}{\partial T}\right)_{v}-p\right] d v \\
& =C_{v} d T-\left[\frac{\partial(p \tau)}{\partial \tau}\right]_{v}
\end{aligned}
$$

in which $\tau=\frac{1}{T}$ 
Integrating eq. (7) gives the difference in internal energy function between two states

$$
\Delta u=\left[-\int_{v_{1}}^{v_{z}}\left(\frac{\partial p T}{\partial \tau}\right)_{v} d v\right]_{T}+\left[\int_{T_{1}}^{T_{2}} C_{v} d T\right]_{v}
$$

The Beattie-Bridgeman equation may be rewritten in the following form:

$$
p=\frac{R\left(1-\frac{c \tau^{3}}{v}\right)}{\tau v^{2}}(v+B)-\frac{A}{v^{2}}
$$

From eq. (9), we have:

$$
-\left[\frac{\partial(p \tau)}{\partial \tau}\right]_{v}=\frac{3 R c \tau^{2}}{v^{2}}+\frac{3 R c B_{0} \tau^{2}}{v^{3}}-\frac{3 R c B_{0} b \tau^{2}}{v^{4}}+\frac{A_{0}}{v^{2}}-\frac{A_{0} a}{v^{3}}
$$

Substituting eq. (10) in eq. (8) and making use of $\mathrm{C}_{\mathrm{v}_{\infty}}$ from spectroscopic data, we have

$$
\begin{aligned}
u & =\frac{1}{778 \times 44}\left\{\frac{A_{0}}{44 v}\left(\frac{a}{88 v}-1\right)-\frac{3(1544) c}{44 \mathrm{vT}^{2}}\left[1+\frac{B_{0}}{88 v}-\frac{B_{0} b}{3(44 v)^{2}}\right]\right\} \\
& +\frac{1}{44}\left(14.2161 \mathrm{~T}-6.53 \times 10^{3} \text { In } \mathrm{T}-\frac{1.41 \times 10^{6}}{\mathrm{~T}}\right)+\mathrm{u}^{\prime \prime}
\end{aligned}
$$

where: $u=$ internal energy $(B t u / l b)$

$$
v=\text { specific volume }\left(\mathrm{ft}^{3} / \mathrm{lb}\right)
$$

$\mathrm{T}=$ absolute temperature $\left({ }^{\circ} \mathrm{R}\right)$

$A_{0}, a, B_{0}, b$ and $c$ as given in eq. (la)

$u^{\prime \prime}=1108.42726$ is arbitrarily chosen to agree with Sweigert

(Ref.I) at 10 psia and $1600 \mathrm{~F}$. 
The enthalpy may be coraputed according to its definition:

$$
h=u+p v
$$

in which: $\mathrm{h}=\mathrm{Btu} / \mathrm{lb}$

$$
\begin{aligned}
& u=B t u / 1 b \text { from eq. } \\
& p=p s i a \\
& v=f t^{3} / 1 b
\end{aligned}
$$

Similarly, the compressibility factor is readily obtained from its definition:

$$
\mathrm{Z}=\frac{\mathrm{pv}}{\mathrm{RT}}
$$

or

$$
\mathrm{Z}=4.126 \frac{\mathrm{pv}}{\mathrm{T}}
$$

in which: $p=$ psia

$$
\begin{aligned}
& v=f t^{3} / 1 b \\
& T=o_{R}
\end{aligned}
$$

\section{Const ruction of the Diagrams}

In the computation of thermodynamic functions, a total of about 7200 combined values of $T$ and $v$ were used as input data. The temperature was in 20 degree intervals from $0 \mathrm{~F}$ to $1600 \mathrm{~F}$. About 80 volumes in convenient intervals were used in the range from .05 to $50 \mathrm{ft}^{3} / 1 \mathrm{~b}$. The constant-volume lines on the T-s diagrams and the constanttemperature lines on the h-s diagram were plotted directly from the computed data. In determining the constant=pressure and the constant= enthalpy lines, cross-plottings on large scale graphs were necessary in order to keep the accuracy within that of chart reading. Because of the large amount of computed data available, no interpolation or 
extrapolation was needed to complete the entire range of the diagrams.

\section{Independent Checks by Joule-Thomson Data and Michels' Isotherm}

Two sources of reliable experimental data are available as independent checks of the accuracy of the completed diagrams. The Joule-Thomson expansion data measured by Roebuck and his co-workers (Ref. 20) were used to check the constant-enthalpy lines in the T-s diagram. Michels' isotherm data (Ref. 4) provided a check of the p-v-t relations. Neither set of data covers the entire range of the diagrams. However, they both provide a useful check in the most critical region, namely, the low temperature and high pressure region in the vicinity of the saturation curve. The checks were made on the large scale diagrams and confirmed the accuracy of the diagrams within 2\%. Figure 9 shows some of the experimental data plotted on a reduced scale T-s diagram for illustration purposes.

Figure 9 also shows a constant-volume line of $0.0718 \mathrm{ft}^{3} / 1 \mathrm{~b}$, which corresponds to an Amagat density of 112. In reference 14 Michels has reported that the Beattie-Bridgeman equation fitted his experiments up to this density with an accuracy of $0.7 \%$. 
CALCULATION OF CONSTANT-VOLUME SPECIFIC HEAT, CONSTANT-PRESSURE SPECIFIC HEAT, SPECIFIC HEAT RATIO, AND THE VELOCITY OF SOUND

\section{A. The Basic Equations}

In the computation of specific heats and their ratios, two basic relations from thermodynamic theory were used, namely,

$$
\left(\frac{\partial^{C} v}{\partial v_{T}}\right)=T\left(\frac{\partial^{2} p}{\partial T^{2}}\right)
$$

and

$$
c_{p}-c_{v}=T\left(\frac{\partial p}{\partial T}\right)_{v}
$$

Detailed derivations of these two relations may be found in reference 19 or in other texts on thermodynamics. With the equation of state avallable, the values of $C_{v}$ and $C_{p}$ may be evaluated from these relations. Thus, by differentiation of the Beattie-Bridgeman equation twice with respect to temperature at constant volume, we have:

$$
\left(\frac{\partial^{2} p}{\partial T^{2}}\right)_{v}=-\frac{6 R c}{v^{2} T^{4}}-\frac{6 R c B_{o}}{v^{3} T^{4}}+\frac{6 B_{o} b R c}{v^{4} T^{4}}
$$

It follows from eq. (14):

$$
\begin{aligned}
& \Delta C_{v}=\left[\int T\left(\frac{\partial^{2} p}{\partial T^{2}}\right) d v\right] \\
& \mathbf{v} \\
& T=\text { const. }
\end{aligned}
$$


The integration in eq. (17) can be carried out by the substitution of eq. (16) in the integrand. Consequently, the constant volume specific heat at a finite volume $v$ can be written as:

$$
C_{v}=\frac{6 R c}{T^{3}}\left(\frac{1}{v}+\frac{B_{0}}{2 v^{2}}-\frac{B_{o} b}{3 v^{3}}\right)+\left(c_{v}\right)_{\infty}
$$

or, in consistent units we have:

$$
C_{v}=\frac{6 R c}{44 \times 778 T^{3}}\left(\frac{1}{44 v}+\frac{B_{o}}{2(44 v)^{2}}-\frac{B_{o} b}{3(44 v)^{3}}\right)+\left(C_{v} b_{0}---(19)\right.
$$

where:

$$
\begin{aligned}
& C_{v} \text { and }\left(C_{v}\right)_{\infty}=B t u / 1 b{ }_{F} \\
& T=o_{R} \\
& v=f t^{3} / 1 b \\
& R=1544 \\
& B_{0}, b \text { and } c \text { as given in eq. (1a) }
\end{aligned}
$$

With $C_{v}$ known, the expression for $C_{\rho}$ may be obtained from eq. (15), which expresses the difference between the specific heats. If the Beattie-Bridgernan equation is written in the form

$$
\mathrm{pv}^{2}=\mathrm{RT}(1-\epsilon)(\mathrm{v}+\mathrm{B})-\mathrm{A}
$$

and differentiated with respect to $T$ at constant pressure, the result is

$$
\left(\frac{\partial v}{\partial T}\right)_{p}=\frac{\left(\frac{2 R c}{v T^{3}}+R\right)\left(v+B_{o}-\frac{B_{o} b}{v}\right)}{2 p v-\left(\frac{R c}{T^{2} v^{2}}\right)\left(v+B_{o}-\frac{B_{o} b}{v}\right)-R T\left(1-\frac{c}{v T^{3}}\right)\left(1+\frac{B_{o} b}{v^{2}}\right)+\frac{a A_{o}}{v^{2}}}
$$


Similarly, by differentiation of eq. (1) with respect to $T$ at constant volume we obtain

$$
\left(\frac{\partial p}{\partial T}\right)_{v}=\frac{R(v+B)}{v^{2}}\left[1+\frac{2 c}{v T^{3}}\right]
$$

Substituting eq. (19) and (20) in eq. (15), the expression for the difference between the specific heats in consistent units becomes:

$C_{p}-C_{v}=\left(\frac{T}{44 \times 778}\right) \frac{\left[\left(\frac{2 R c}{44 v T^{3}}+R\right)\left(44 v+B_{0}-\frac{B_{0} b}{44 v}\right)\right]^{2}}{288 p(44 v)^{3}-\frac{R c}{T^{2}}\left(44 v+B_{0}-\frac{B_{0} b}{44 v}\right)-R T\left(1-\frac{c}{44 v T^{3}}\right)\left[(44 v)^{2}+B_{0} b\right)+a A 0}$

where: $\quad C_{p}$ and $C_{v}=B t u /{ }_{1 b}{ }^{\circ}$

$$
\begin{aligned}
& T=o_{R} \\
& p=p s i a \\
& v=\mathrm{ft}^{3} / \mathrm{lb} \\
& \mathrm{R}=2544
\end{aligned}
$$

$A_{0}, a, B o, b$ and $c$ as given in eq. ( $1 a$ )

Note that the term $\mathrm{p}$ appears in the right hand side of eq. (21). For each given $(T, v)$ input, the term $p$ shall be computed from the equation of state.

The ratio of specific heats, $\gamma$, is readily obtained from its definition:

$$
\gamma=c_{p} / c_{v}
$$

The velocity of sound with no absorptions (low frequencies) is given by the expression:

$$
v_{s}^{2}=g\left(\frac{\partial p}{\partial \rho}\right)_{s}=-g v^{2}\left(\frac{\partial p}{\partial v}\right)_{s}
$$


By using the usual equations relating the partial derivatives of thermodynamic functions, eq. (23) may be changed to the form:

$$
v_{\mathrm{s}}^{2}=g \gamma\left(\frac{\partial p}{\partial \rho}\right)_{\mathrm{T}}=-g \gamma \mathrm{v}^{2} \quad\left(\frac{\partial \mathrm{p}}{\partial \mathrm{v}}\right)_{\mathrm{T}}
$$

The term $\frac{\partial p}{\partial v}$ in eq. (24) can be evaluated from the equation of state. For the Beattie-Bridgeman equation we have:

$$
\begin{aligned}
& \mathrm{V}_{\mathrm{s}}^{2}=\frac{\gamma}{44}\left[\mathrm{RT}-\left(\frac{\mathrm{Rc}}{\mathrm{T}^{2}}-\mathrm{RTB}_{0}+\mathrm{A}_{\mathrm{O}}\right) \frac{2}{44 \mathrm{v}}-\left(\frac{\mathrm{RCBO}}{\mathrm{T}^{2}}+\mathrm{RTB}_{\mathrm{O}} \mathrm{b}-\mathrm{A}_{\mathrm{O}} \mathrm{a}\right)\right. \\
& \left.\frac{3}{(44 \mathrm{v})^{2}}+\frac{4 \mathrm{~B} \mathrm{bRc}}{\mathrm{T}^{2}(44 \mathrm{~V})^{3}}\right]
\end{aligned}
$$

where: $\quad V_{s}=$ velocity of sound, $\mathrm{ft} / \mathrm{sec}$

$$
\begin{aligned}
& \gamma=\text { specific heat ratio, from eq. (22) } \\
& g=32.2 \mathrm{ft} / \mathrm{sec}^{2} \\
& T=o_{R} \\
& v=\mathrm{ft}^{3} / 1 \mathrm{~b} \\
& R=1544
\end{aligned}
$$$$
A_{0}, a, B_{0}, b \text { and } c \text { as given in eq. (1a) }
$$

In the region near the critical point, the computed velocity in accordance with eq. (25) decreases steadily along an isotherm as pressure increases, goes through a minimum, and starts to increase. This indicates the correct trend as evidenced by the experimental data of Anderson and Delsasso (Ref. 44), and Richardson (Ref. 45). However, in some instances the computed values disagree with the experimental data by as much as $30 \%$. At first it was suspected that the discrepancies might be caused by the mathematical difficulties inherent in the basic equation $(24)$. In the neighborhood of the critical point, 
the isothermal slope approaches zero and $\gamma$ approaches infinity. Since eq. (24) involves the product of these two quantities, erroneous results could be obtained because of this mathemathical indeterminacy. An alternate form was tried for the velocity equation:

$$
v_{s}^{2}=-g v^{2}\left(\frac{\partial p}{\partial v}\right)_{T}+\frac{g v^{2} T}{c_{v}}\left(\frac{\partial p}{\partial T}\right)_{v}^{2}
$$

This expression can be easily derived by using the usual relations among the partial derivatives of thermodynamic functions. It is general and exact in theory just as is eq. (24). In the neighborhood of the critical point, the first term in the right-hand side of eq. (26) approaches zero, while the second term remains finite. By using the Beattie-Bridgeman equation, eq. (26) becomes:

$$
v_{s}^{2}=\varnothing+\Psi
$$

where: $\phi=\frac{g}{44}\left[R T-\left(\frac{R C}{T^{2}}-R T B_{0}+A_{0}\right) \frac{2}{44 V}\right.$

$$
\begin{aligned}
& \left.-\left(\frac{R c B_{o}}{T^{2}}+R T B_{o} b-A_{o} a\right) \frac{3}{(44 v)^{2}}+\frac{4 B_{o} b R c}{T^{2}(44 v)^{3}}\right] \\
& \Psi=\frac{\mathrm{gTv}^{2}}{778 \mathrm{C}} \mathrm{v}\left[\frac{\mathrm{R}\left(44 \mathrm{v}+\mathrm{B}_{0}-\frac{\mathrm{B}_{\mathrm{Q}} \mathrm{b}}{44 \mathrm{v}}\right)\left(1+\frac{2 \mathrm{c}}{44 \mathrm{vT}}\right)}{(44 \mathrm{v})^{2}}\right]^{2} \\
& v_{s}=f t / s e c \\
& C_{v}=B t u / 1 b{ }^{\circ} F \\
& T=O R \\
& v=f t^{3} / 1 b \\
& g=32.2
\end{aligned}
$$




$$
\begin{aligned}
& R=1544 \\
& A_{0}, a, B_{0}, b \text { and } c \text { are the same as given in eq. (1a). }
\end{aligned}
$$

The computed results of eq. (27) were identical to those of eq. (25). This indicates that the suspected mathematical indeterminacy is not the cause of the difficulty. Another attempt was made to use Michels' isotherms (Ref. 14) Instead of the Beattie-Bridgeman equation for evaluating the velocity. Michels' isotherms are given in the series form:

$$
p v=A+(B+\beta) d+C d^{2}+Z d^{3}+D d^{4}+E d^{6}+F d^{8}
$$

in which $d$ is the density expressed in Amagat units. The coefficients ( $A, B, \beta$, etc.) are functions of temperature. The computed results of velocity were far less satisfactory than those obtained from the Beattie-Bridgeman equation when compared with the experimental data. It is interesting to note that the Michels isotherms, eq. (28), and the Beattie-Bridgeman equation are both accurate to better than $1 \%$ in representing $p-v-t$ relations within their range of validity, but differ In yielding the velocity values by as much as $50 \%$. The completed chart for the velocity of sound was finally obtained by extrapolating and smoothing the computed values from the Beattie-Bridgeman equation to fit the available experimental data in the region near the critical point.

\section{B. Discussion}

The accuracy of the computed values of $\mathrm{Z}, \mathrm{Cp}, \mathrm{Cv}$, and $\gamma$ in this report is of high order. The use of the Beattie-Bridgeman equation was not incidental. Extensive review and comparison of both experimental and 
computed data available were made before the justification for its use was confirmed. The computed values were directly plotted on large scale charts as isotherms with pressure as a variable. One cross-plotting was required to transfer them to the final form (isobars with temperature as variable). A close examination of the final charts indicates that, in general, the errors inherent in the graphical representation of the computed values are in the order of magnitude of $\pm 2 \%$.

In the case of the velocity of sound, it is estimated that the accuracy of the computed values in this report is about $\pm 3 \%$. However, for the region in which the temperature is below about $350 \mathrm{~F}$ and pressure greater than 1000 psia, a possible error of $\pm 10 \%$ must be assumed in the application of the velocity values to engineering design. 
-

0 
CALCULATION OF ABSOLUTE VISCOSITY, HEAT CONDUCTIVITY, AND PRANDTL NUMBER

\section{A. The Basic Equations}

The equation for viscosity of $\mathrm{CO}_{2}$ at low pressure is given by Keyes in a three-constant empirical form:

$$
10^{5} \eta_{0}=\frac{a_{0} \sqrt{T}}{1+a^{T / 10} a_{1}}
$$

where: $\eta_{0}=$ absolute viscosity at zero pressure (poise)

$$
\begin{aligned}
& T=\text { absolute temperature }\left({ }^{\circ} \mathrm{K}\right) \\
& \tau=\text { reciprocal of } \mathrm{T}\left(1 /{ }^{\circ} \mathrm{K}\right) \\
& a_{0}=1.554 \\
& a=246 \\
& a_{1}=3
\end{aligned}
$$

The effect of pressure on viscosity has been correlated by means of density and the reciprocal of absolute temperature. It may be expressed in the form:

$$
\log \left(\Delta \eta \cdot 10^{5}+7\right)=.8451+.4667 \times 10^{3} \rho \tau
$$

in which: $\Delta \eta=\eta-\eta_{0}$

$$
\begin{aligned}
& \eta=\text { absolute viscosity at the desired pressure (poise) } \\
& \rho=\text { density (gm/cc) } \\
& \log =\text { common logarithm (base } 10)
\end{aligned}
$$

The equation for heat conductivity of $\mathrm{CO}_{2}$ at low pressure is given by Keyes in a similar form of equation: 


$$
10^{5} \cdot \lambda_{0}=\frac{c_{0} \sqrt{T}}{1+c^{T} / 10^{C_{1}^{T}}}
$$

where: $\lambda_{0}=$ heat conductivity at zero pressure $\left(\mathrm{cal} / \mathrm{cm}-\mathrm{sec}-{ }^{\circ} \mathrm{C}\right)$

$$
\begin{aligned}
& C_{0}=3.333 \\
& C=4433 \\
& C_{1}=10 \\
& T, T \text { same as in eq. (29). }
\end{aligned}
$$

The pressure effect of heat conductivity is given by the equation:

$$
\log \left(\Delta \lambda \cdot 10^{5}+4\right)=.6021+.405 \times 10^{3} \rho \tau
$$

in which: $\Delta \lambda=\lambda-\lambda_{0}$

$$
\lambda=\text { heat conductivity at the desired pressure }\left(\mathrm{cal} / \mathrm{cm}-\mathrm{sec}-{ }^{\circ} \mathrm{C}\right)
$$

Other symbols and their units are the same as given in eq. (30)

The Prandtl number is readily obtained from its definition:

$$
P_{r}=\frac{C p^{\mu}}{K}
$$

in which: $C_{p}=$ specific heat at constant pressure $\left(B t u / 1 b{ }^{\circ} F\right)$ as obtained from previous calculations.

$$
\mu=\text { absolute viscosity in } 1 \mathrm{~b} / \mathrm{hr}-\mathrm{ft}
$$$$
(=241.9 \times \eta)
$$$$
\mathrm{K}=\text { heat conductivity in } \mathrm{Btu} / \mathrm{hr}-\mathrm{ft}-{ }^{\circ} \mathrm{F}
$$$$
(=241.9 \times \lambda)
$$ 


\section{B. Discussion}

The range of validity of eq. (29) for viscosity at low pressure is from $-103 \mathrm{~F}$ to $2582 \mathrm{~F}$ (Ref. 2). For the range of interest in our report ( $0 \mathrm{~F}$ to $1500 \mathrm{~F}$ ), eq. (29) fits the experimental data of Vasilesco (Ref. 46) with an accuracy of $\pm 1 \%$. At 1 atm, viscosity values from the full-scale chart of our work agree with Nuttall's tabulated values (Ref. 22) within 3\%. It may be noted that Nuttall's values at low pressure were based on the theoretical work of Hirschfelder et al. (Ref. 23, 24).

According to Keyes (Ref. 3), the correlation for the pressure effect on viscosity, eq. (30), is valid for densities from 0 to $0.66 \mathrm{gm} / \mathrm{cc}$ (i.e., specific volumes from infinity down to $.0243 \mathrm{ft}^{3} / 1 \mathrm{~b}$ ). Keyes used equations of this type in correlating experimental viscosity data of many gaseous substances, including steam (a highly polar substance). In the case of steam Keyes was accurate within $\pm 1 \%$ in his correlation of the most recent Russian data up to $1112 \mathrm{~F}$ and 2850 psia (Ref. 47).

However, a check of the viscosity values of $\mathrm{CO}_{2}$, as computed from eqs. (29) and (30) with some of the experimental data reported in various sources, indicates considerable discrepancies. An extensive correlation of all the available experimental data was therefore considered necessary. The data taken for correlation are listed in Table 1.

It was found that considerable discrepancies existed among the experimental data reported by the various investigators. For example, at $68 \mathrm{~F}$ and in the pressure range of $800 \mathrm{psia}$, the viscosity values 
measured by Phillips are about 20\% lower than those of Schröer and Becker. It is interesting to note that, when correlated on the basis of density, the data obtained by the rolling-ball method (Schröer and Becker; Kiyama and Makita) are invariably on the higher side as compared with those obtained by the transpiration method (Phillips; Comings, Mayland, and Egly). This fact has also been noted by Michels et al. (Ref. 50).

The combination of eq. (29) and (30) as given in our report represents the best possible correlation of the experimental data listed in Table 1. The accuracy of the computed viscosity values here is within $\pm 1 \%$ for low pressure conditions (0 to 200 psia). For pressures up to about 1000 psia, $\pm 5 \%$ accuracy is estimated. For pressures above 1000 psia, $\pm 10 \%$ accuracy must be assumed in engineering applications. It is known that the effect of pressure on viscosity is less significant at higher temperatures. (The most significant effect of pressure on viscosity occurs near the saturated region.) The percentage of accuracy given above was based on the correlation of the available low temperature data (maximum $572 \mathrm{~F}$, Table 1). For higher temperatures, the percentage of accuracy should be better, although no experimental data are available for a quantitative evaluation. Table 2 shows some computed values of eq. (29) and (30) as compared with Michels' data (Ref. 50) at one isotherm.

Eq. (31) for the heat conductivity of $\mathrm{CO}_{2}$ at low pressure fits the experimental data in the range from $-117 \mathrm{~F}$ to $607 \mathrm{~F}$. Keyes (kef. 3) has found a deviation of $\pm 5 \%$ when the equation is extrapolated to $1892 \mathrm{~F}$. At $1 \mathrm{~atm}$, heat conductivity values from the full-scale chart 
of our work agree with Nuttall's tabulated values (kef. 32) within $3 \%$ up to $620 \mathrm{~F}$, which is the maximum temperature listed in Nuttall's report. Compared with the smoothed experimental data of Stolyarov et al. (Ref. 5l) at $1 \mathrm{~atm}$, the disagreement varies from 3 to $6 \%$ for the temperature range up to $392 \mathrm{~F}$.

Eq. (32) for the effect of pressure on heat conductivity of $\mathrm{CO}_{2}$ is valid for densities from 0 to $0.45 \mathrm{gm} / \mathrm{cc}$ (i.e., specific volumes from infinity to $\left..0356 \mathrm{ft}^{3} / 1 \mathrm{~b}\right)$. Between temperatures of $32 \mathrm{~F}$ to $302 \mathrm{~F}$, Keyes has estimated $5 \%$ accuracy or better for eq. (32). The accuracy of this equation is rather uncertain when extrapolated to higher temperatures because of the complete lack of experimental data for comparison. However, based on the experience with steam, an equation of the type similar to eq. (32) extrapolates well into the higher temperature region (Ref. 3,47 ). It is believed that an overall accuracy of $\pm 10 \%$ can be assumed for the results of our work in temperatures beyond $302 \mathrm{~F}$. Table 3 gives some pertinent comparisons with Stolyarov's data. It should be noted that his data are widely scattering over his reported temperature range. This is not at all surprising in view of the experimental difficulties involved.

Other published data available for comparing the effects of pressure on heat conductivity are Gamson's (Ref. 36). His semi-theoretical work, which is supposedly applicable to all gaseous substances, has been discussed briefly in Section III, paragraph $E$ of our report. A check of the heat conductivity values for $\mathrm{CO}_{2}$ obtained from Gamson's data shows considerable discrepancies, which range from about $4 \%$ at low pressure and temperature $(0$ psia, $200 \mathrm{~F})$ to as much as $34 \%$ at high 
pressure and temperature $(2000 \mathrm{psia}, 1500 \mathrm{~F})$. The reliability of the results obtained from a generalized treatment such as Gamson's is indeed questionable. They serve, at best, as an indication of the order of magnitule of heat conductivity values for a particular gaseous substance.

It is obvious from the above discussion that considerable experimental work remains to be done in order to reliably ascertain the thermal conductivity of carbon dioxide, particularly at high pressure and temperature conditions.

The computed values of Prandtl numbers in this work agree with those of Hilsenrath et al. (Ref. 10) within $2 \%$ for temperatures up to $620 \mathrm{~F}$ and 1 atm pressure, which are the maximum temperature and the only pressure condition given in Hilsenrath's report. No other published data are available for comparison. 
CONSTRUCTION OF THE T-S AND THE h-s DIAGRAMS

IN THE SATURATED REGION

In the saturated region, the tabulated data of Sweigert as reported in the Chemical Engineers' Handbook (Ref. 52) were taken for comparison with those of Newitt et al. (Ref. 11). For the temperature range from $-50 \mathrm{~F}$ to the critical temperature, the values of vapor pressure and the specific volumes of the saturated liquid and vapor were in good agreement. The maximum discrepancy is less than $2 \%$ at temperatures near the critical point. For lower temperatures, the agreement is within $1 \%$. In the case of enthalpy and entropy values, the two sets of data have different reference states. When transformed to a comparable basis, the agreement was found to be within $1 \%$, which was considered very satisfactory.

It may be noted that the work of Newitt et al. was based on the vapor pressure data of Meyer and Van Dusen (Ref. 53) and the latent heat and specific volume data of Michels et al. (Ref. 54). These data were obtained with the greatest possible care and are generally considered the most accurate available. Newitt's tabulated values were used to construct the $T-s$ and the h-s diagrams for the saturation region. The enthalpy and entropy values have been transformed to the same reference state as that employed in the gaseous phase of this report, namely, $h_{0}=180 \mathrm{Btu} / \mathrm{lb}$ and $\mathrm{S}_{\mathrm{o}}=1 \mathrm{Btu} / \mathrm{lb}-\mathrm{o}_{\mathrm{R}}$, both at $32 \mathrm{~F}$ saturated liquid (Ref. I). 
The constant quality lines were obtained by using the formula

$$
s=S_{f}+x S_{f g}
$$

in which

$$
\begin{aligned}
& S=\text { the entropy corresponding to a specified quality } x \text { at a } \\
& \text { temperature } T_{1} \\
& S_{f}=\text { entropy of the saturated liquid at } T_{1} \\
& S_{f g}=S_{g}-S_{f}= \\
& \text { difference in entropy between the saturated } \\
& \text { vapor and the saturated liquid at } T_{I}
\end{aligned}
$$




\section{REFERENCES}

1. Thermodynamic Properties of Gases, Carbon Dioxide, by R. L. Sweigert, P. Weber, and R. $L$. Allen, Industrial and Engineering Chemistry, Vol. 38, No. 2 (1946), p. 185

2. The Heat Conductivity, Viscosity, Specific Heat, and Prandtl Numbers for Thirteen Gases, by F. G. Keyes, Project Squid Technical Report No. 37, Massachusetts Institute of Technology, 1952

3. Private Communication (April, 1957), with Professor F. G. Keyes of Massachusetts Institute of Technology, Cambridge, Mass.

4. Isotherms of $\mathrm{CO}_{2}$ between $0^{\circ}$ and $150^{\circ} \mathrm{C}$ and Pressure from 16 to 250 Atmospheres, by A. Mi.chels and C. Michels, Proceedings of the Royal Society, Vol. 153A (1935), pp. 201-214

5. Isotherms of $\mathrm{CO}_{2}$ between 70 and 3000 Atmospheres, by A. Michels, c. Michels, and H. H. Wonters, Proceedings of the Royal Society, Vol. $153 \mathrm{~A}(1935), \mathrm{pp}$. 214-224

6. Compressibility of Gases at High Temperatures IV Carbon Dioxide, by K. E. MacCormack and W. G. Schneider, Journal of Chemical Physics, Vol. 18 (1950), pp.1269-1272

7. P-v-t Data for $\mathrm{CO}_{2}$ up to $1000^{\circ} \mathrm{C}$ and 1400 Bars, by G. C. Kennedy, Progress Report No. 2 under NOrd - 10449, Task 5 with Harvard University, 1953

8. P-v-t Relations in $\mathrm{CO}_{2}$ at Elevated Temperatures and Pressures by G. C. Kennedy, American Journal of Science, Vol. 252 (1954), pp. $225-241$

9. Ze1tschrift für die gesamte Kälte-Industrie, Vol 1 (1929), p. 1 Zeitschrift für technische Physik, Vol. 10 (1929), p.'99 by R. PIank and J. Kuprianoff

10. Tables of Thermal Properties of Gases, by $J$. Hilsenrath, et al., National Bureau of Standards Circular 564, U.S. Government Printing Office, 1955

11. Carbon Dioxide, by D. M. Newitt, M. V. Pai, N. R. Kuloor, and J. A. W. Huggill, in the book edited by F. Din Thermodynamic Functions of Gases, Vol. 1, Butterworths Scientific Publications, London, 1956

12. Thermodynamic Properties of Carbon Dioxide up to $1000^{\circ} \mathrm{C}$ and 1400 Bars - I. Entropy, Enthalpy, and Isobaric Heat Capacity, by D. Price, NAVORD Report 3846, U.S. Naval Ordnance Laboratory, 1954 
13. A New Equation of State for Fluids. Im Application to Gaseous Ethyl Ether and Carbon Dioxide, by J. A. Beattie and $0 . C$. Bridgeman, Journal of the American Chemical Society, Vol. 49 $(1927), \mathrm{p} . \overline{1665}$

14. Series Evaluation of the Isotherm Data of $\mathrm{CO}_{2}$ between 0 and $150^{\circ} \mathrm{C}$ and up to $3000 \mathrm{~atm}$, by $\mathrm{A}$. Michels, and C. Michels, Proceedings of the Royal Society, Vol. $160 \mathrm{~A}$ (1937), pp. 348-357

15. An Overall Test of Equations of State of Fluids, by N. Davy and B. M. Bell, British Journal of Applied Physics, Vol. 9 (1958), p. 27

16. An Equation of State for Gases, by G. J. Su and C. H. Chang, Industrial Engineering Chemistry, Vol. 38 (1946), p. 800

17. International Critical Tables, portion by, A. Leduc, Vol. 5, McGraw-Hill Book Co., 1929, p. 83

18. Carbon Dioxide, by E. L. Quinn and C. L. Jones, American Chemical Society, Monograph 72, New York, Reinhold Publishing Corp., 1936

19. The Specific Heats of Certain Gases over Wide Ranges of Pressures and Temperatures, by F. O. Ellenwood, N. Kulik, and N. R. Gay, Cornell University Engineering Experimental Station, Bulletin No. 30,1942

20. The Joule-Thomson Effect of Carbon Dioxide, by J. R. Roebuck, T. A. Murrell, and E. E. Miller, Journal of The American Chemical Society, Vol. 64 (1942), p. 400

21. Brief Review of Available Data on the Dynamic Viscosity and Thermal Conductivity for Twelve Gases, by G. A. Hawkins, Transactions of The American Society of Mechanical Engineers, Vol. $70(194,8), \mathrm{pp} .655-659$

22. Table 13.39 Carbon Dioxide, Coefficients of Viscosity, by R. L. Nuttall, The NBS-NACA Tables of Thermal Properties of Gases, Dec. 1950

23. The Transport Properties for Non-Polar Gases, by J. O. Hirschfelder, R. B. Bird, and E. L. Spotz, Journal of Chemical Physics, Vol. $16(1948)$, p. 968

24. Viscosity and Other Physical Properties of Gases and Gas Mixtures, by J. O. Hirschfelder, R. B. Bird, and E. L. Spotz, Transactions

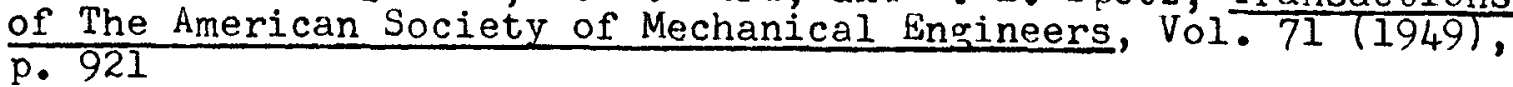

25. The Viscosity of Carbon Dioxide, by P. Phillips, Proceedings of the Royal Society, Vol. A-87 (1912), p. 48 
26. Ueber die Zahigkeit Vershiedener Kältemittel in flussigen und dampfformigen Zustand in Abhängigkeit von Druck und Temperatur, by $\mathrm{H}$. Stakelbeck, Zeitschrift für die gesamte Kälte-Industrje, Vol. $40(1 9 3 3 \longdiv { , p . 3 3 }$

27. Viscosity of Gases at High Pressures, by E.W. Coming, B.J. Mayland, and R.S. Egly, University of IIlinois Engineering Experimental Station, Bulletin Series $354,7,1944$

28. The Mathematical Theory of Non-Uniform Gases, by S. Chapman, and T.G. Cowling, Cambridge University Press, 1939

29. Viscosity Behavior of Gases, by L.A. Bromley, and C.R. Wilke, Industrial and Engineering Chemistry, Vol. 43 (1951), p. 1641

30. The Lennard-Jones 12:6 Potential and the Viscosity of Gases, by E. Whalley and W.G. Schneider, Journal of Chemical Physics, Vol. $20(1952)$, p. 657

31. The Viscosity of Carbon Dioxide, Ammonia, Acetylene, Argon and Oxygen under High Pressures, by R. Kiyama, and T. Makita, Review of Physical Chemistry of Japan, Vol. 22, No. 22 (1952), p. 49

32. Table 13.42 Carbon Dioxide, Thermal Conductivity, by R.I. Nuttall, The NBS-NACA Tables of Thermal Properties of Gases, Dec. 1950

33. Thermal Conductivity and the Accomodation Coefficient of Carbon Dioxide, by C.T. Archer, Philosophical Magazine Vol. 19 (1935) p. 901

34. Hot-Wire Method for the Thermal Conductivity of Gases, by G.G. Sherrat, and E. Griffiths, Philosophical Magazine, Vol. 27 $(1939)$, p. 68

35. An Improved Hot-Wire Cell. for Accurate Measurements of Thermal Conductivities of Gases over a Wide Temperature Range, by H.L. Johnston, and E.R. Grilly, Journal of Chemical Physics, Vol. 14 $(1946)$, p. 219

36. A Generalized Thermal Conductivity Correlation for Gas State, by B.W. Gamson, Chemical Engineerjing Progress, Vol. 45 (1949), pp. $154-159$

37. In the "Technical Section", by O.A. Uyehara, and K.M. Watson, National Petroleum News, Oct. 4, 1944

38. Kungljga Svenska Vetenskapsakademiens Handligar, by D. Enskog, Vol. 63, (1922)

39. The Specific Heats of Gases, by, J.R. Partington, and W. G. Shilling, Ernest Benn Ltd., London, 1924 
40. Measurements of Sound-Velocities in Air, Oxygen and Carbon Dioxide at Temperatures from $900^{\circ} \mathrm{C}$ to $1200^{\circ} \mathrm{C}$, with Special Reference to the Temperature-Coefficients of Molecular Heats, by F.F. King, and J.R. Partington, Philosophical Magazine, Vol. 9 (1930), p. 1020

41. The Determination of the Specific Heat of Gases at High Temperatures by the Sound Velocity Method, II - Carbon Dioxide, by G.G. Sherratt, and E. Griffiths, Proceedings of the Royal Society, Vol. A-156 (1936), p. 504

42. Annalen der Physik, by H.O. Kneser, Vol. 11 (1931), p. 761

43. Ratio of Specific Heats of Air, $\mathrm{N}_{2}$, and $\mathrm{CO}_{2}$ as a Function of Pressure by the Ultrasonic Method, by J.C. Hubbard, and A.H. Hodge, Journal of Chemical Physics, Vol. 5 (1937), p. 978

44. The Propagation of Sound in Carbon Dioxide Near the Critical Point, by N.S. Anderson, and L.P. Delsasso, Journal of the Acoustic Society of America, Vol. 23, No. 4 (1951), p. 423

45. Absorption and Velocity of Sound in Vapors, by E.G. Richardson Reviews of Modern Physjes, Vol. 27, No. 1 (1955), p. 15

46. Recherches experimentales sur la viscosite des gaz temperatures elevees, by V. Vasilesco, Annales de Physique, Vol. 20 (1945), p. 292

47. Brief Notes and Comments on the Presently Existing Steam Data, by F.G. Keyes, Presentation at 5th International Conference on Properties of Steam, July 1956, London, England

48. The Viscosity of Carbon Dioxide in the Critical Region, S.N. Naldrett, and 0 . Maass, Canadian Journal of Research, Vol. 18B $(1940)$, p. 322

49. Untersuchungen über den Kritischen Zustand, by E. Schröer, and G. Becker, Zeitschrift für Physiologische Chemie, Vol. Al73 (1935), p. 178

50. The Viscosity of Carbon Dioxide between $0^{\circ} \mathrm{C}$ and $75^{\circ} \mathrm{C}$ and at Pressures up to 2000 Atmospheres, by A. Michels, A. Botzen and W. Schuurman, Physica, Vol. 23 (1957), p. 95

51. Thermal Conductivity of Five Gases up to $200^{\circ} \mathrm{C}$ and 300 Atmosphere, in Russian, by E.A. Stolyarov and V.V. Ipateev, Zhur. Fiz. Khim., Vol. 24 (1950), p. 166

52. Chemical Engineers' Handbook, by J.H. Perry, 3rd ed., McGraw-Hill, $1950, \mathrm{p} .254$ 
53. The Vapor Pressure of Liquid and Solid Carbon Dioxide, by C. H. Meyers, and M. S. Van Dusen, Journal of Research of The National Bureau of Standards, Vol. 10 (1933), p. 381

54. The Isotherms of $\mathrm{CO}$ in the Neighborhood of the Critical Point and Round the Coexistence Line, by A. Michels, B. Blaisse, and C. Michels, Proceedings of the Royal Society, Vol. A-160 (1937), p. 358

55. Sulla conductivitia termica dei gas e dei vapori, by C. Codegone, Accad. Sci. Torino, Vol. $86(1951-52)$, p. 288 
0

0 
IX

FIGURES 


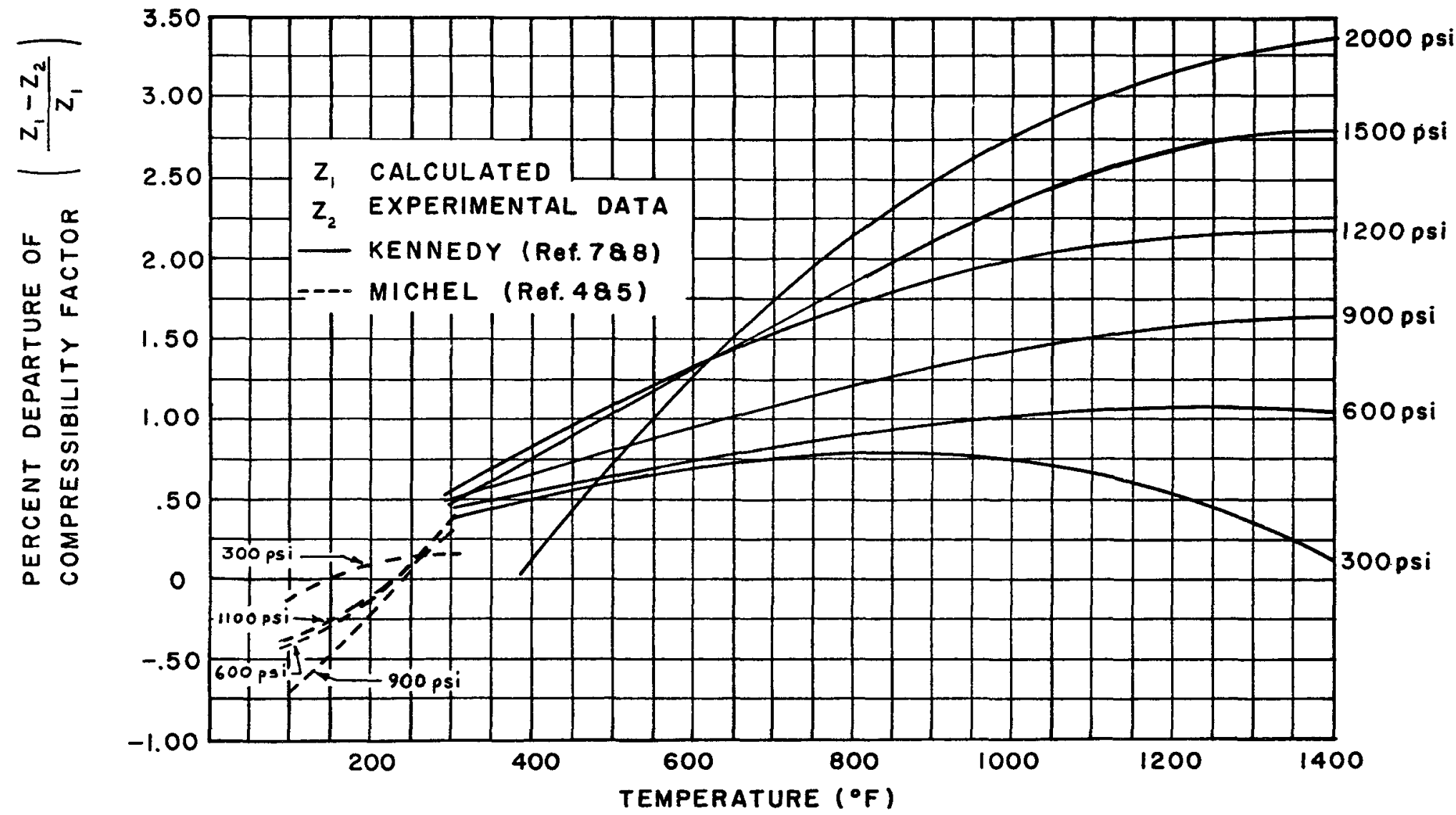

FIG.I COMPARISON OF COMPRESSIBILITY DATA FOR $\mathrm{CO}_{2}$ 


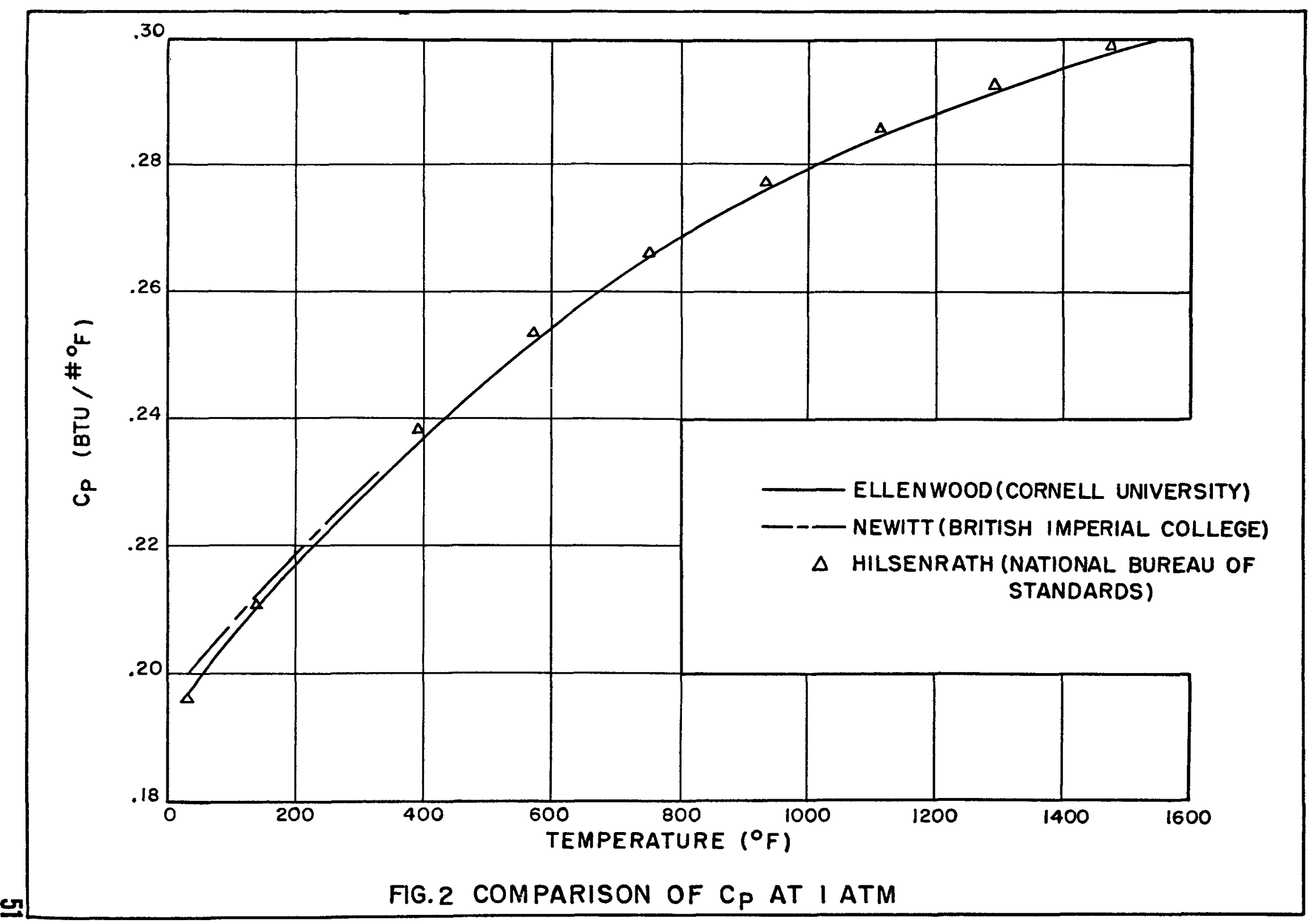




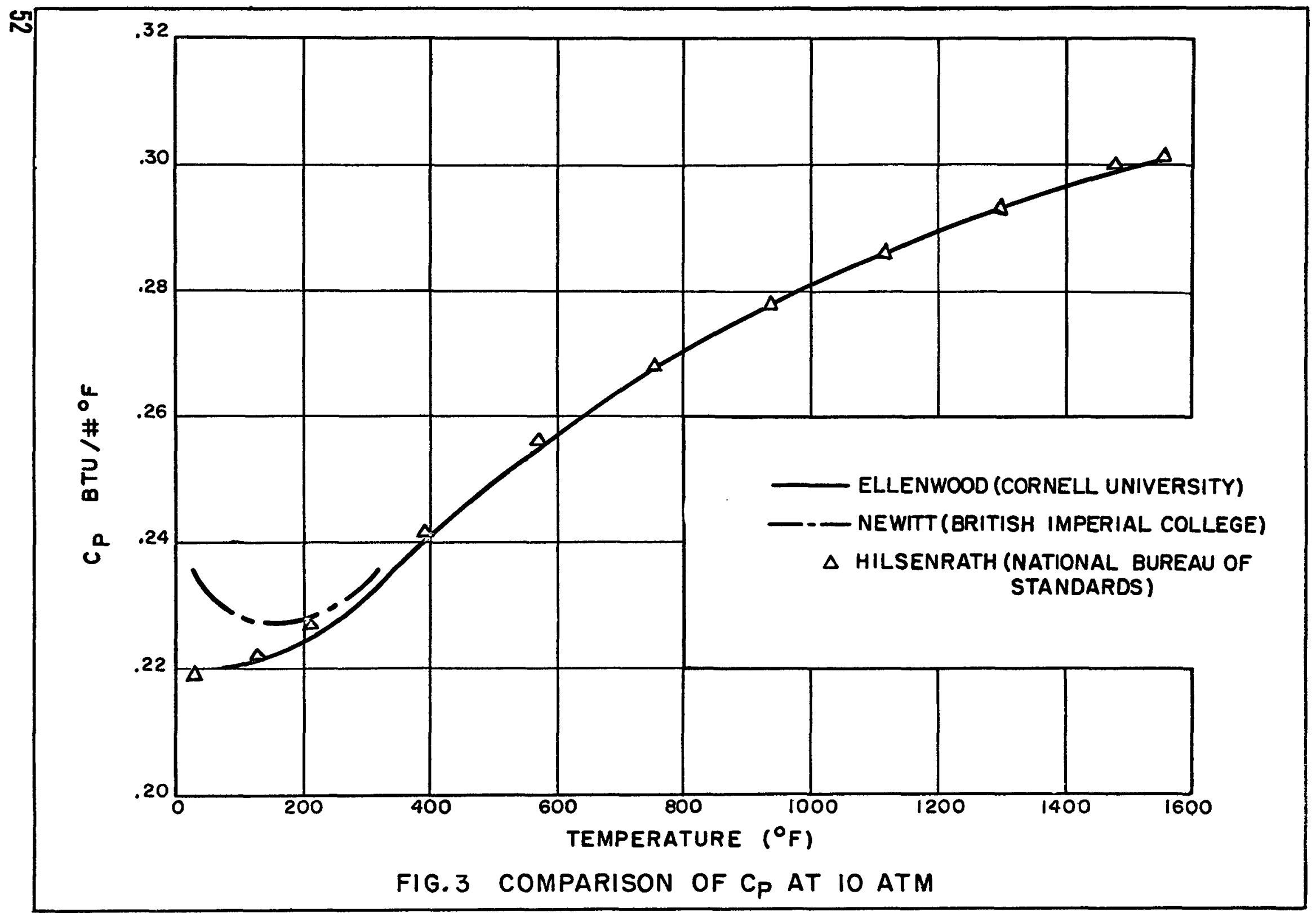




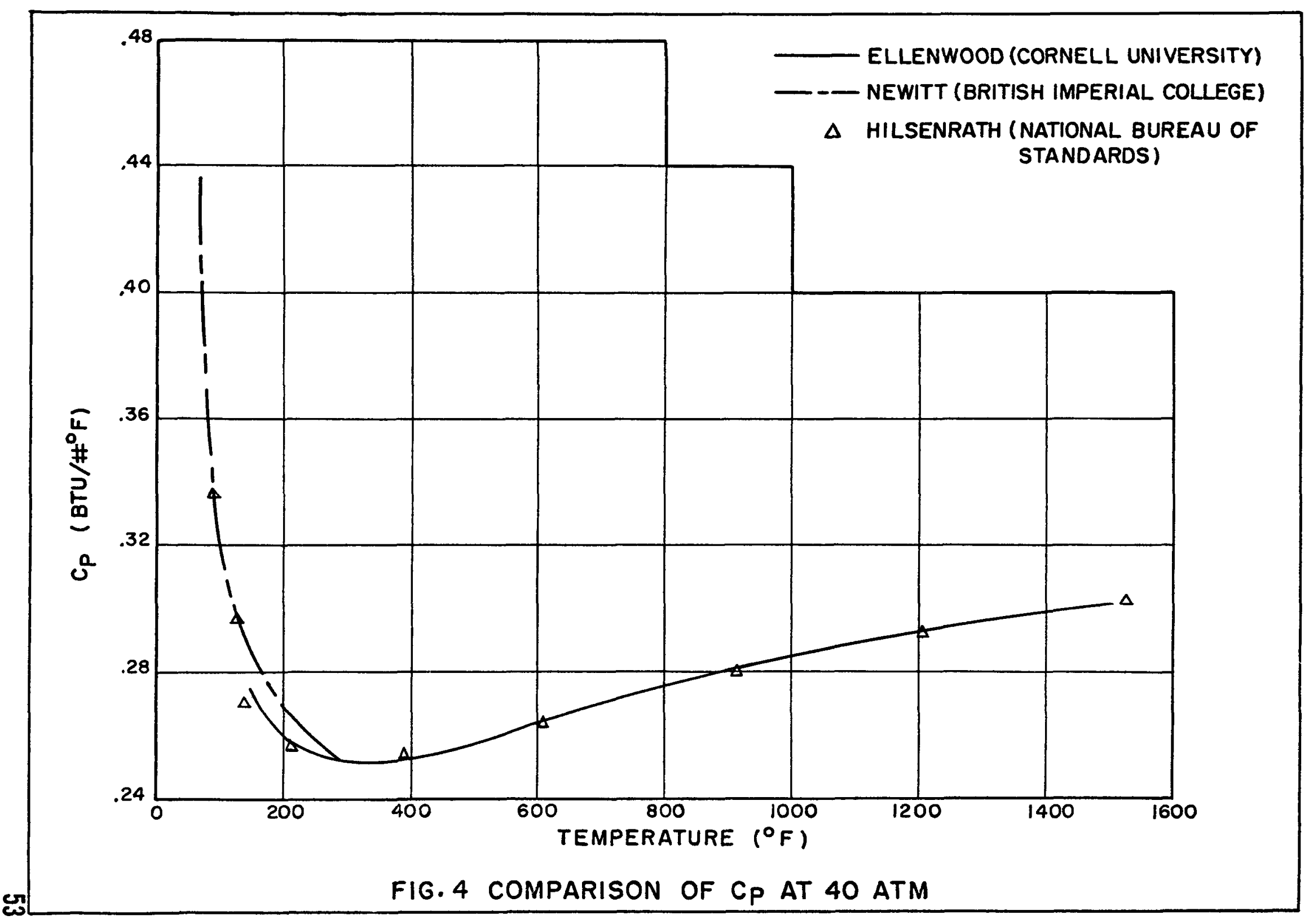




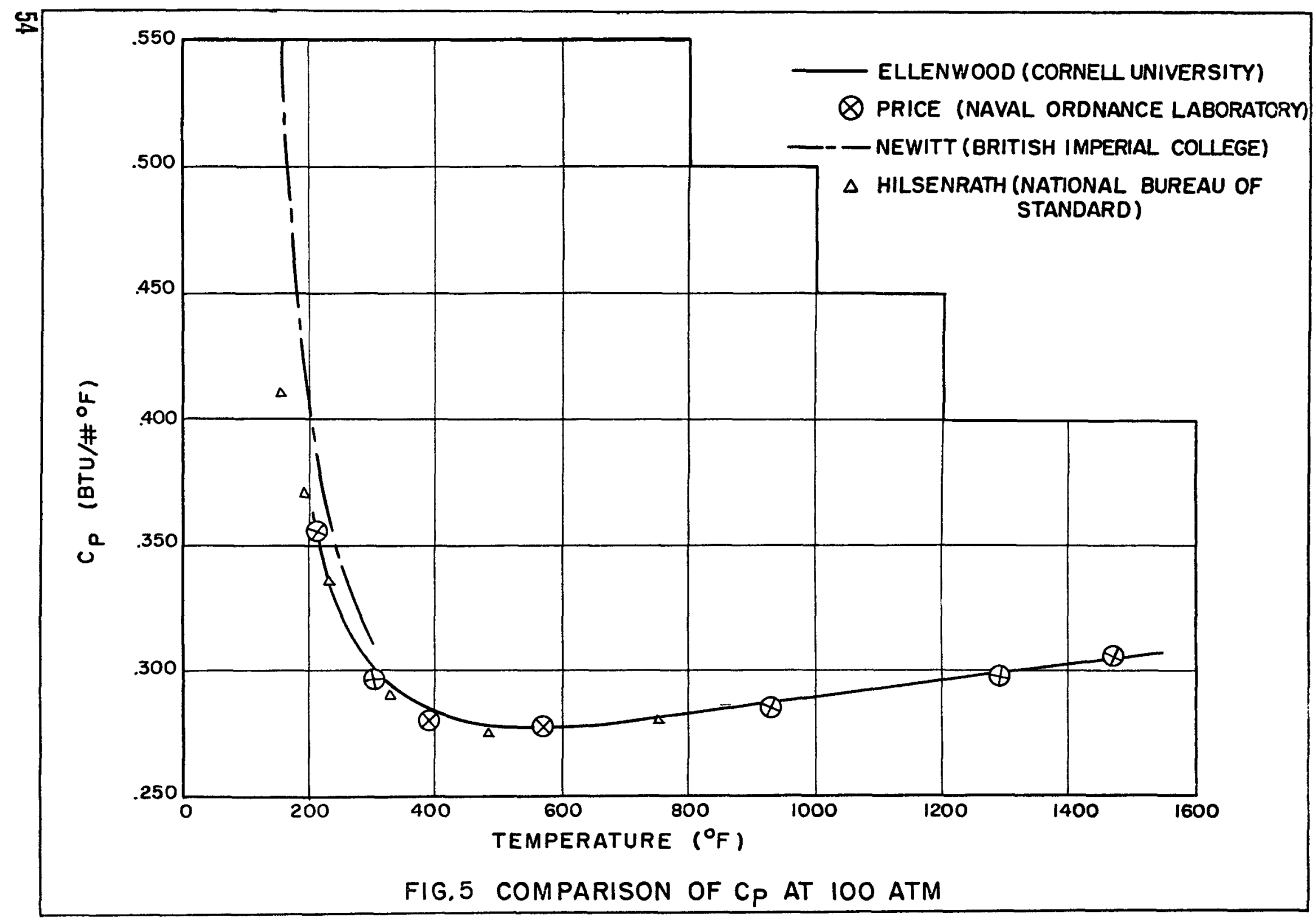




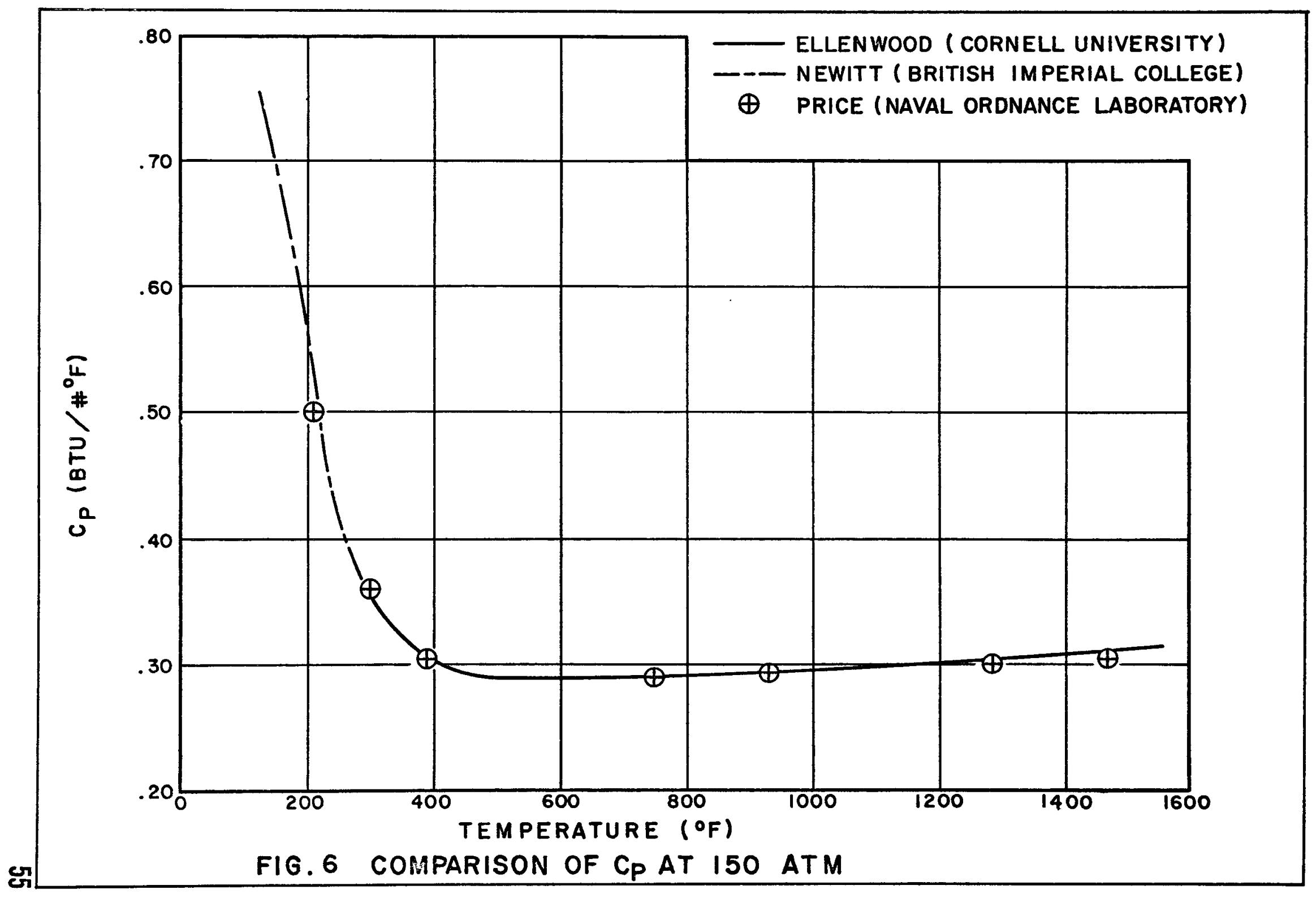




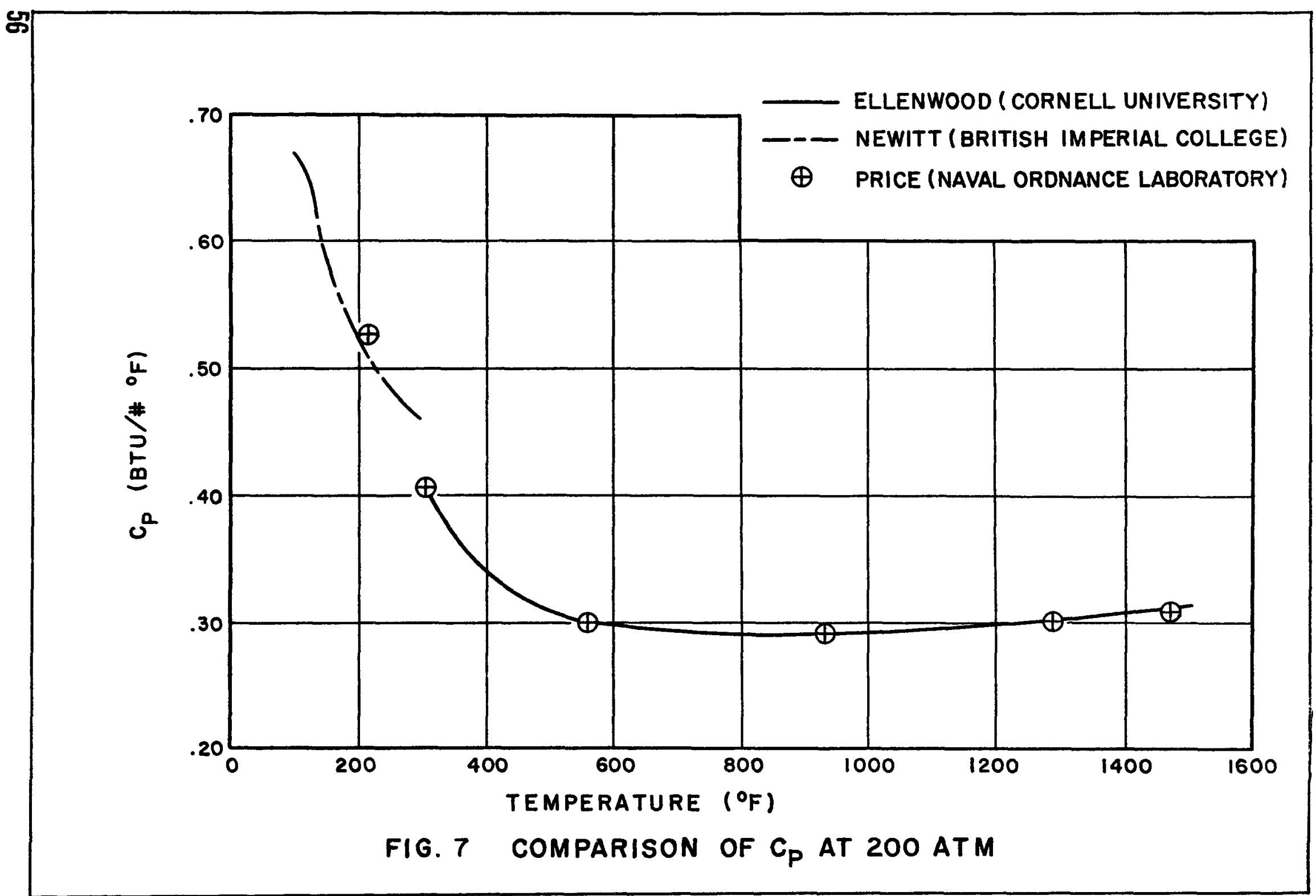




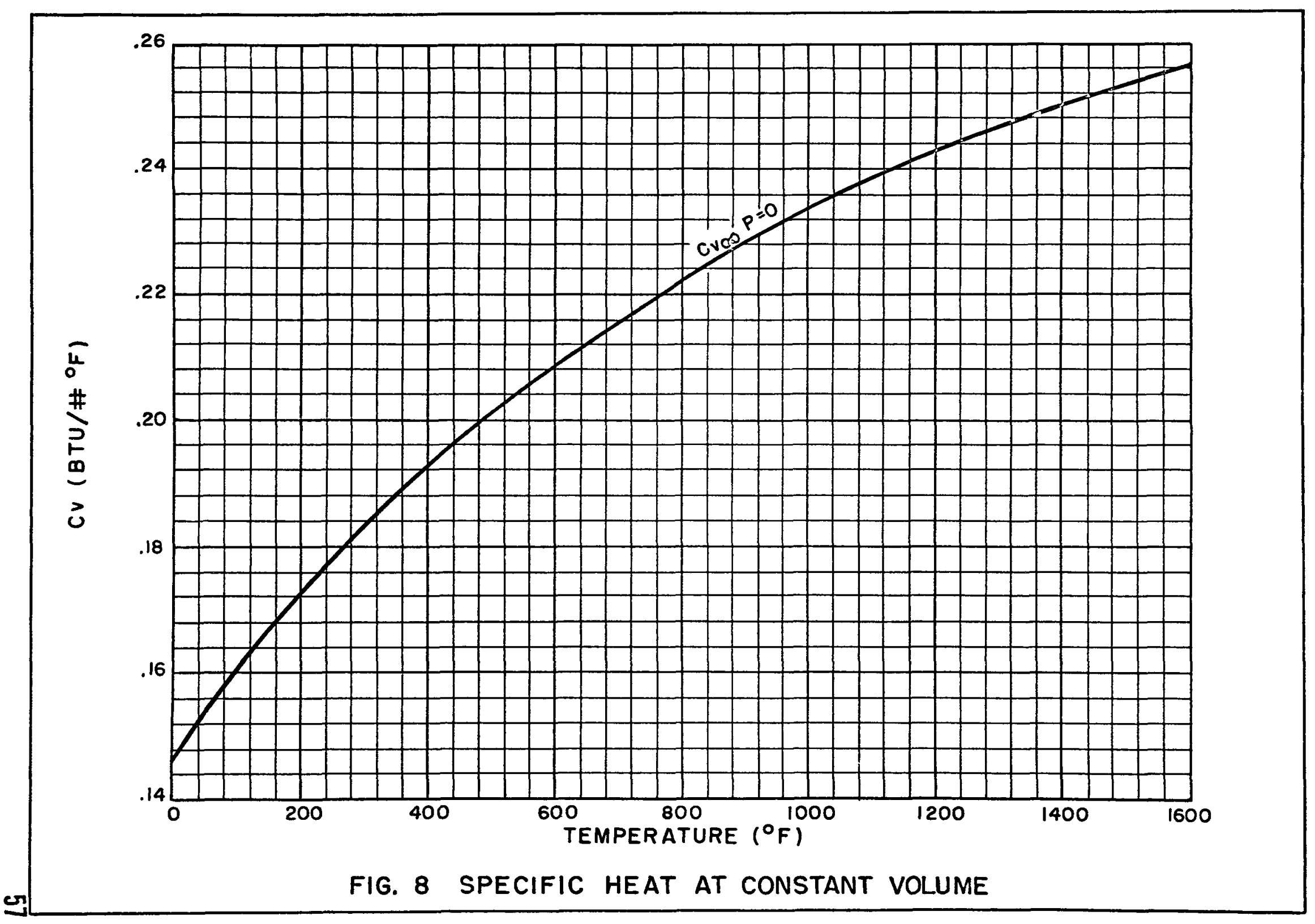




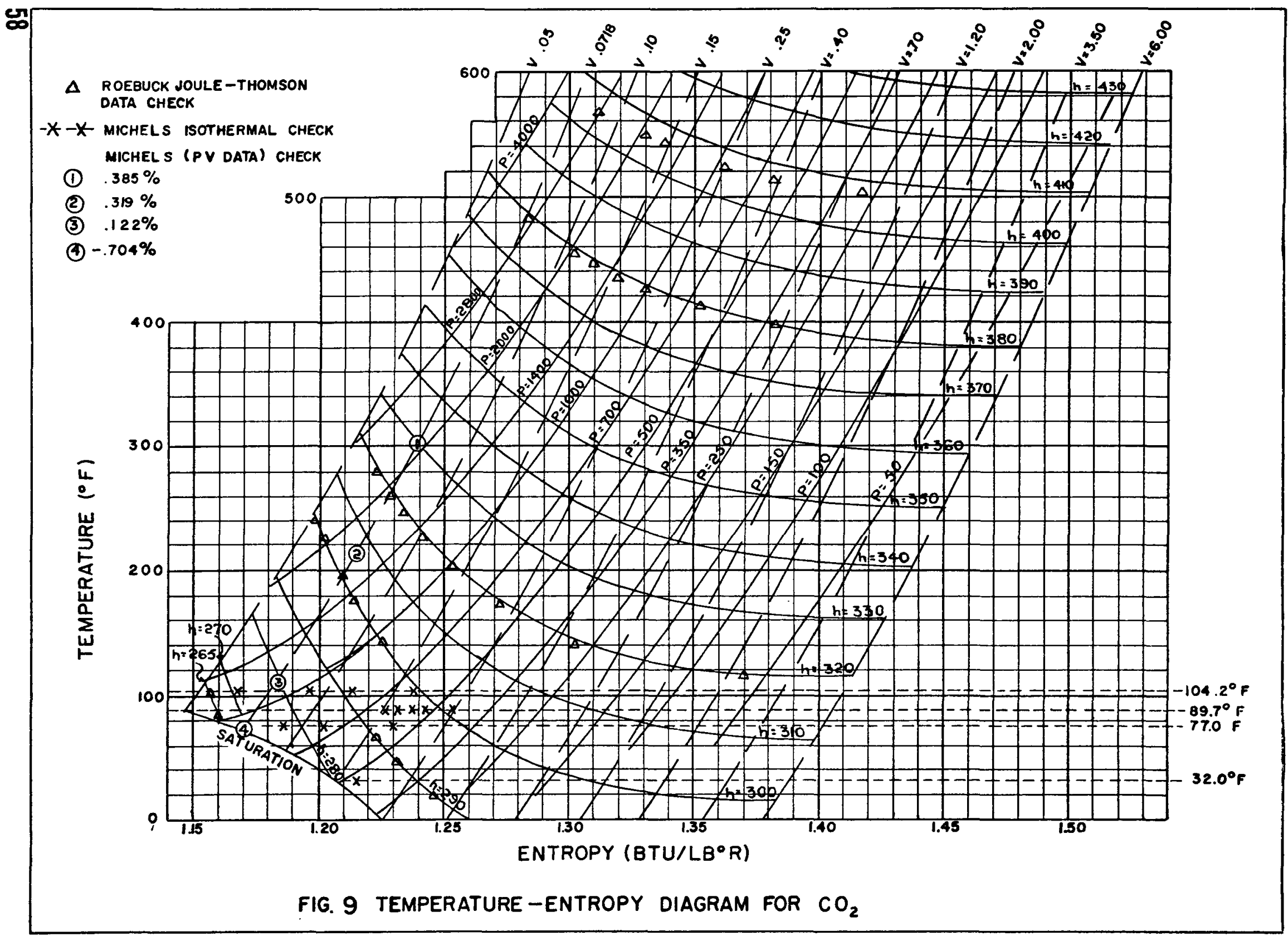

FIG. 9 TEMPERATURE -ENTROPY DIAGRAM FOR $\mathrm{CO}_{2}$ 


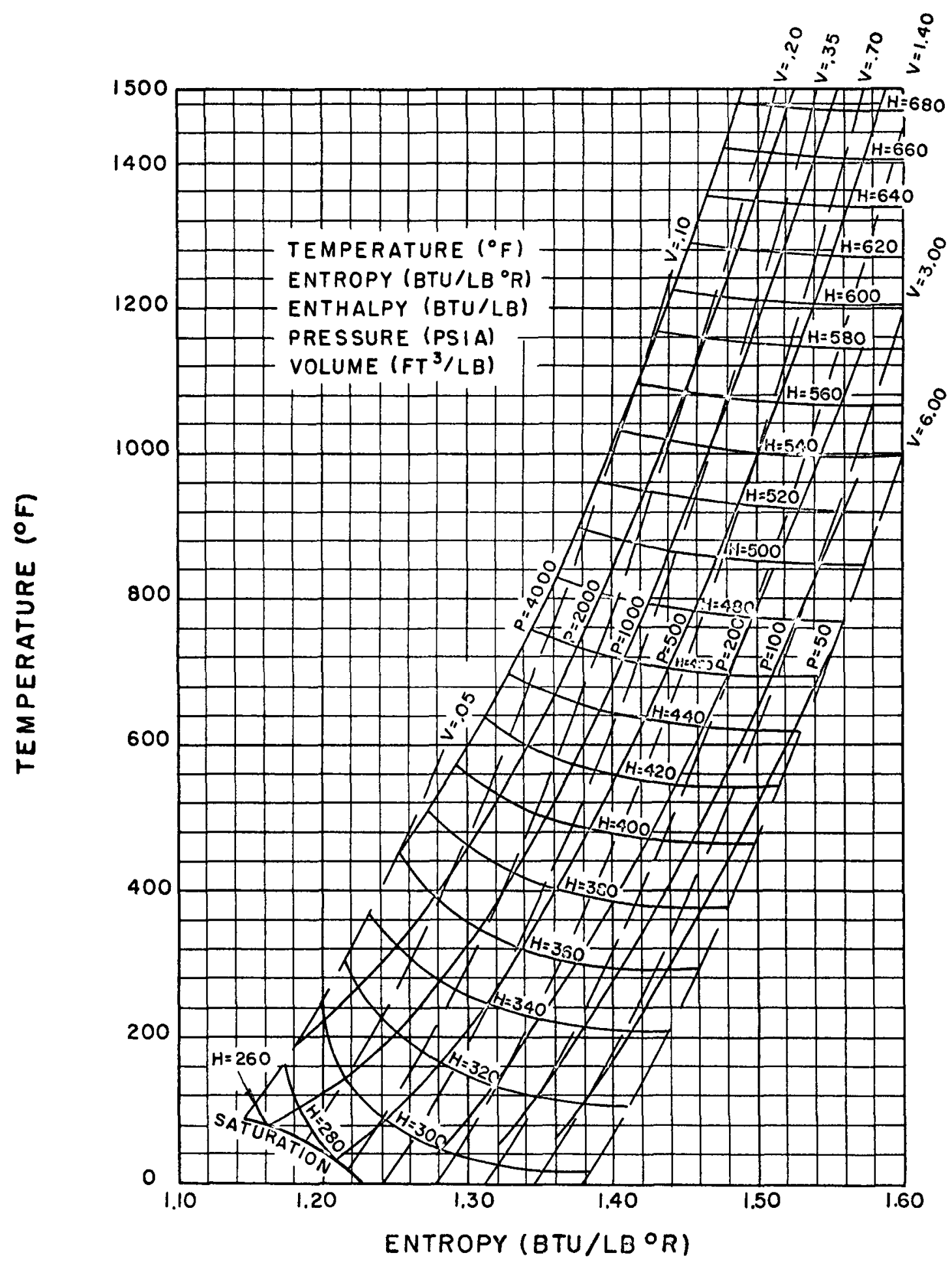

FIG.10 TEMPERATURE-ENTROPY DIACRAM FOR $\mathrm{CO}_{2}$ 


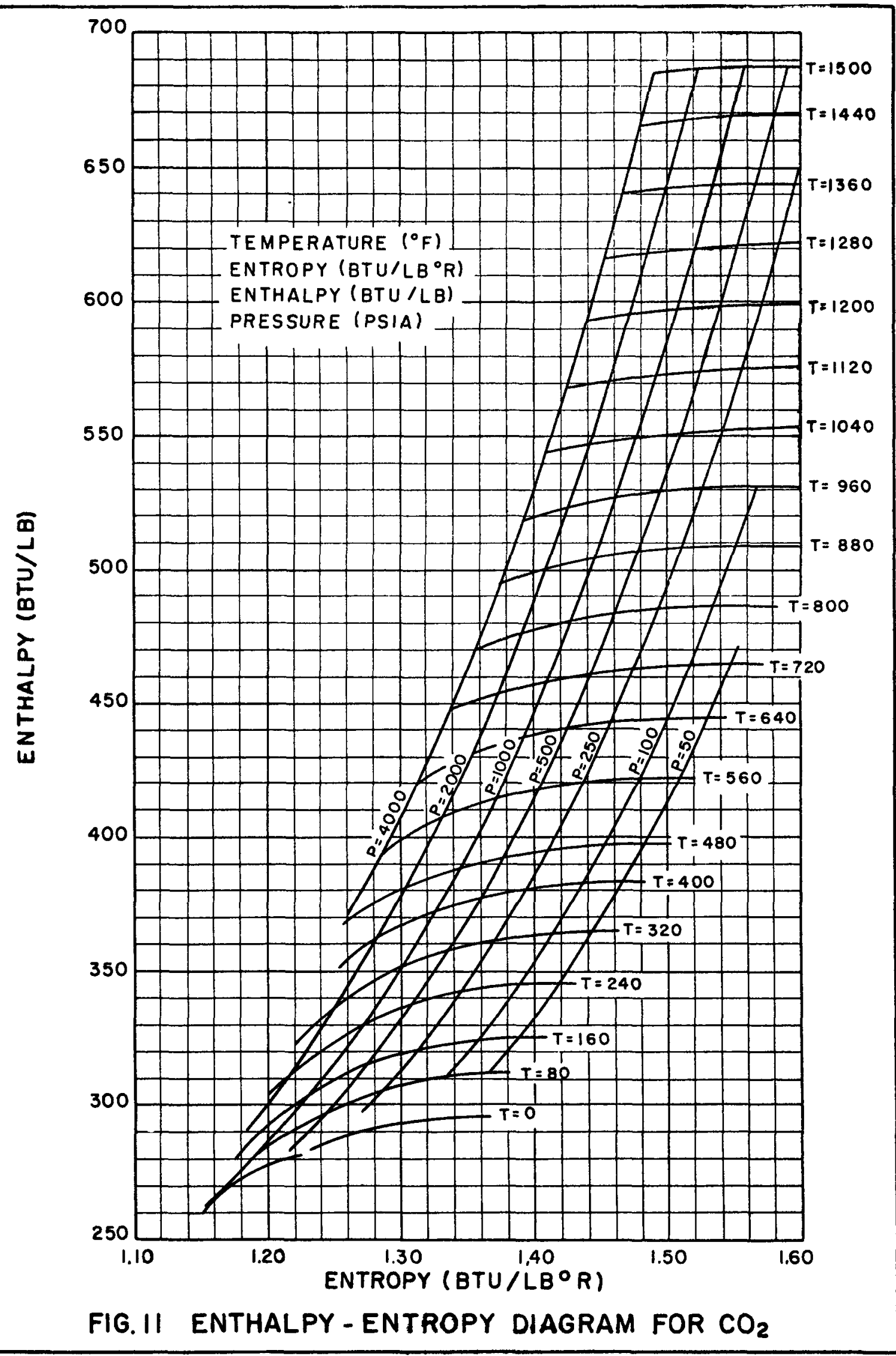




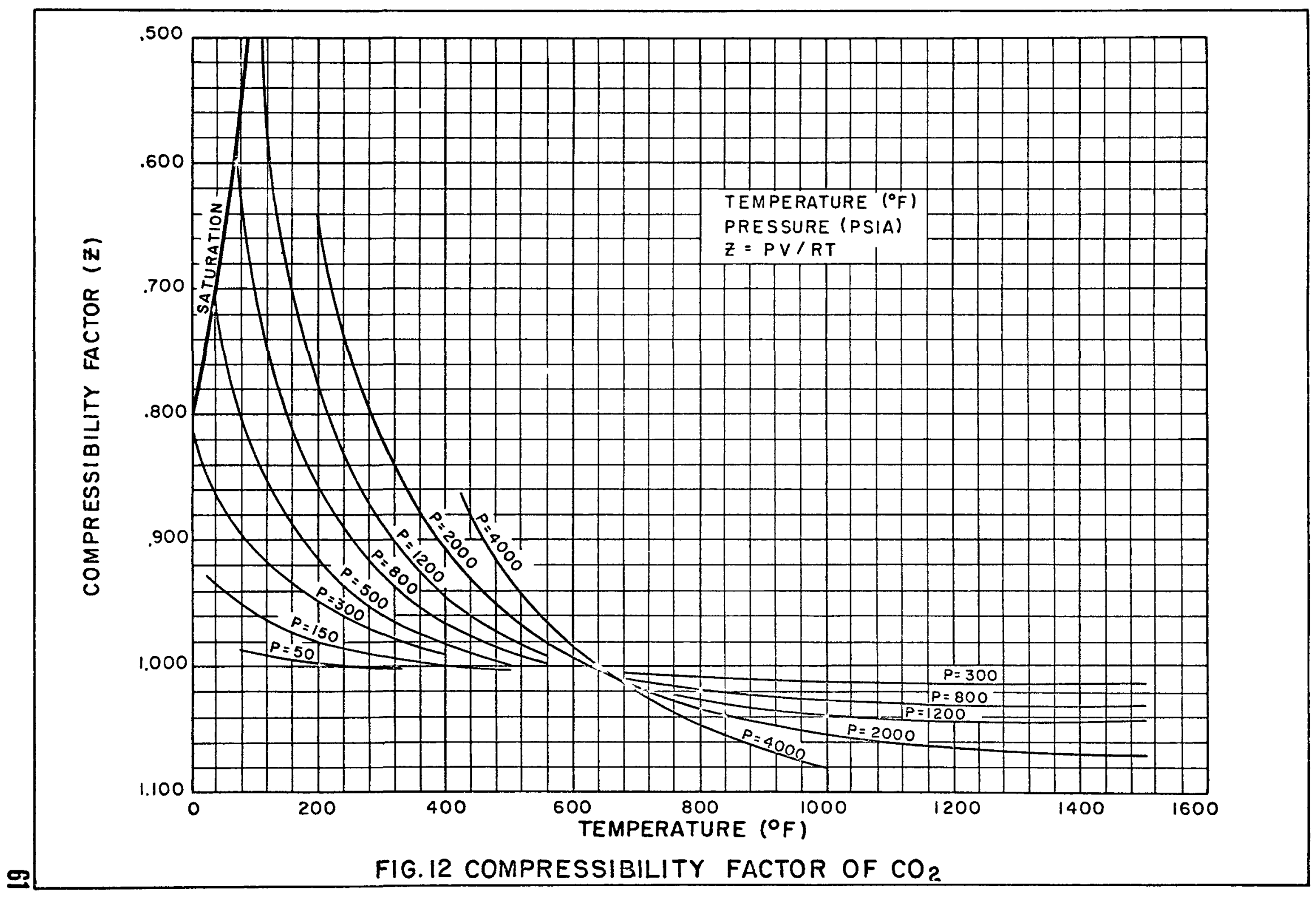




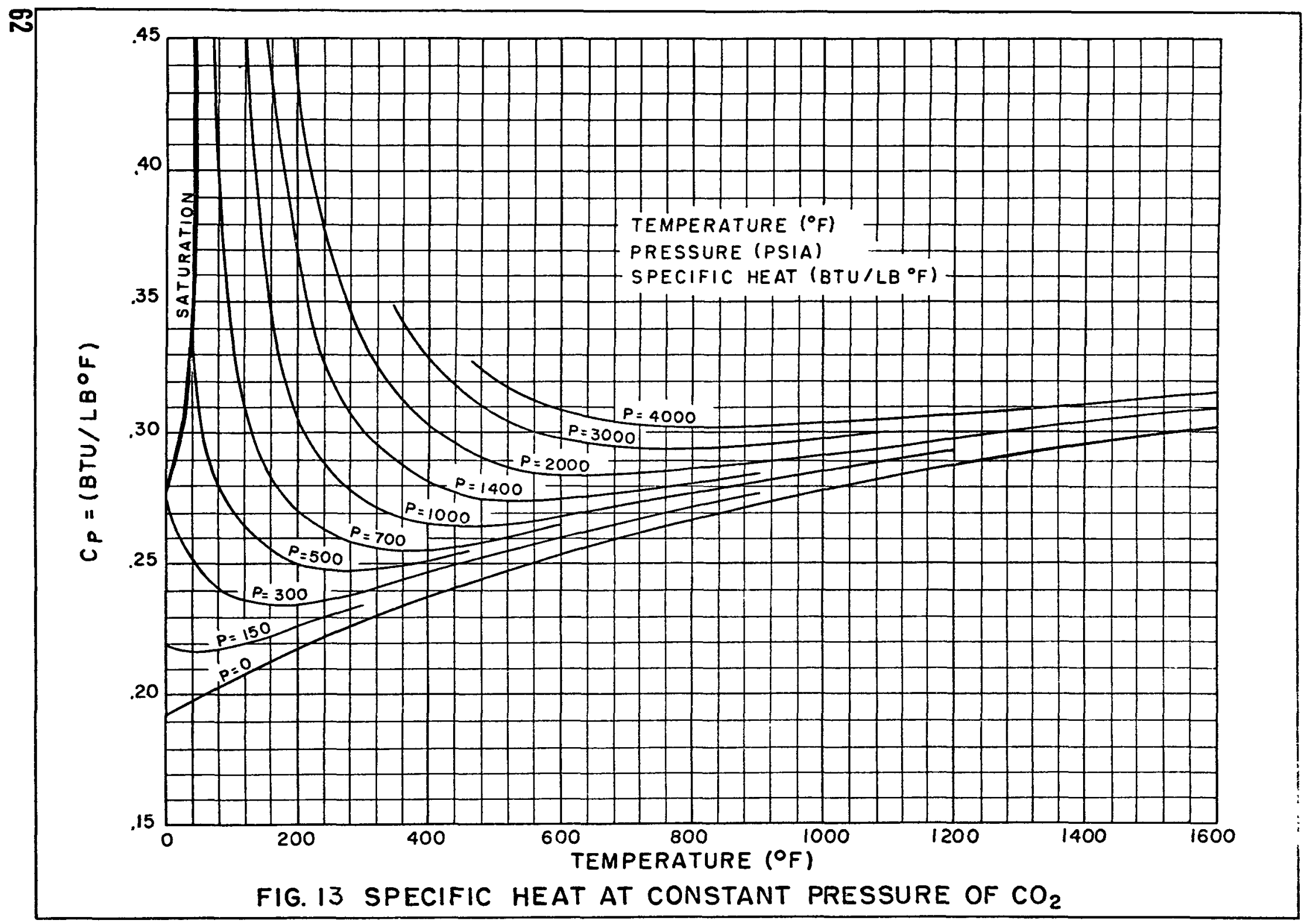




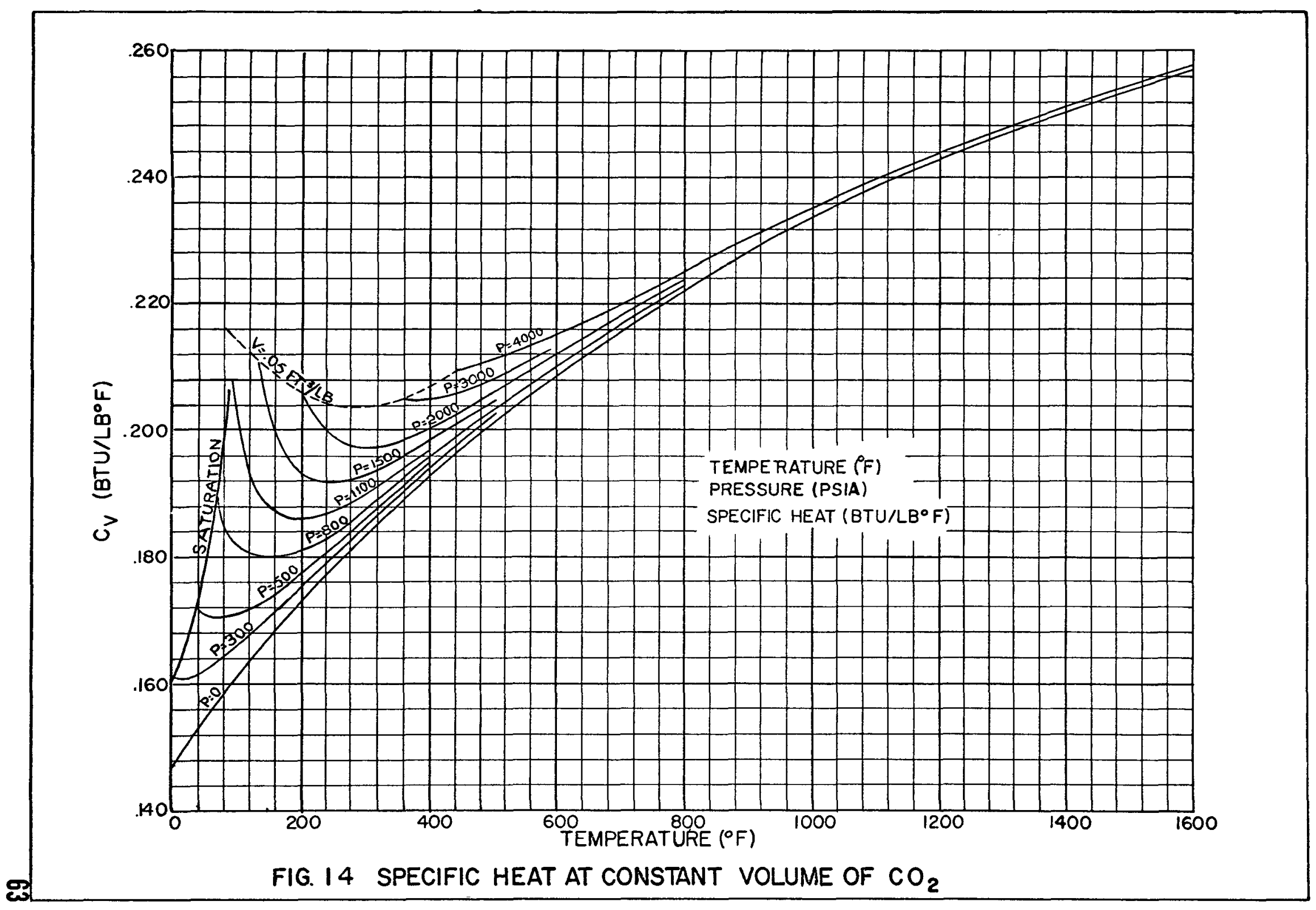




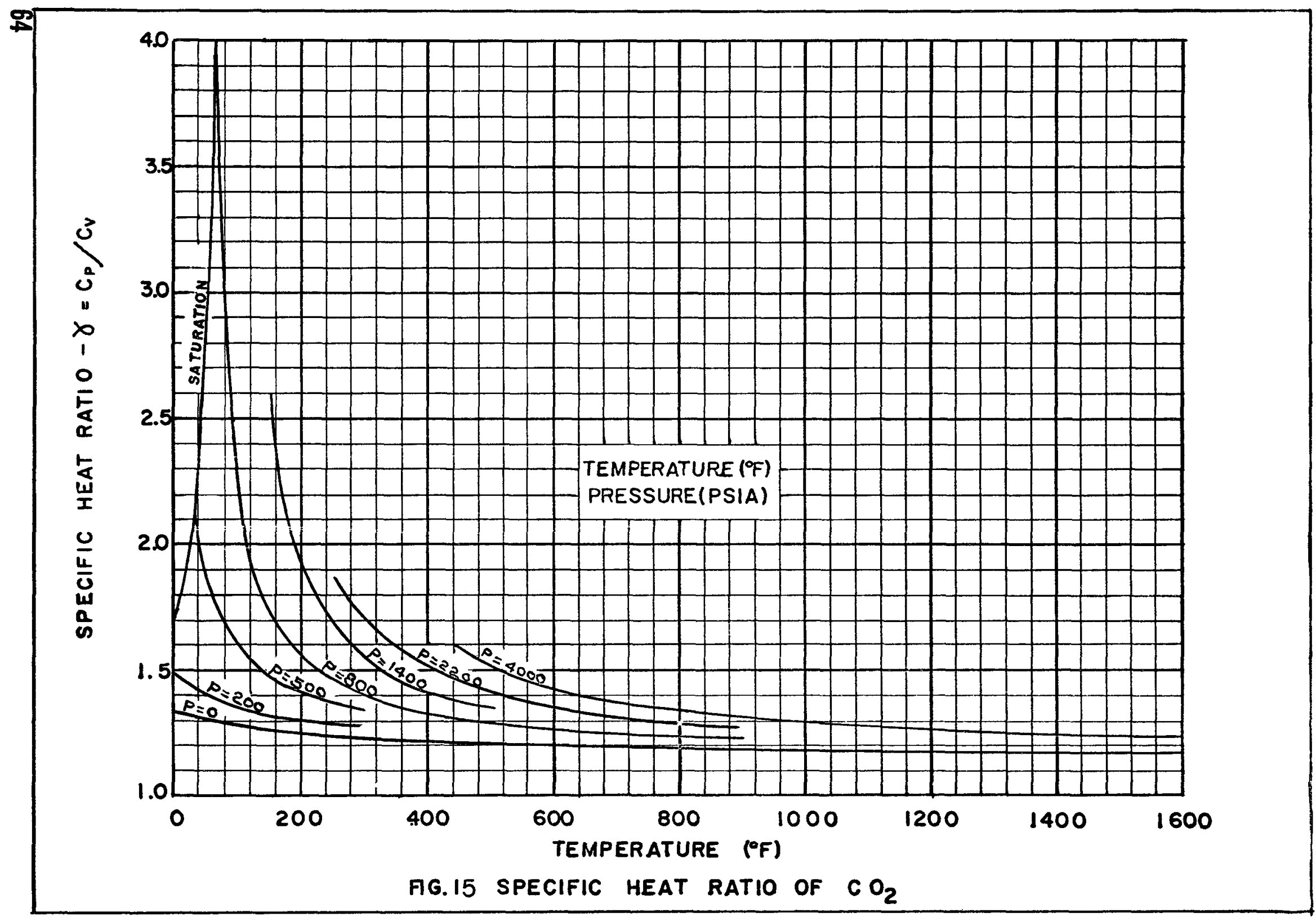




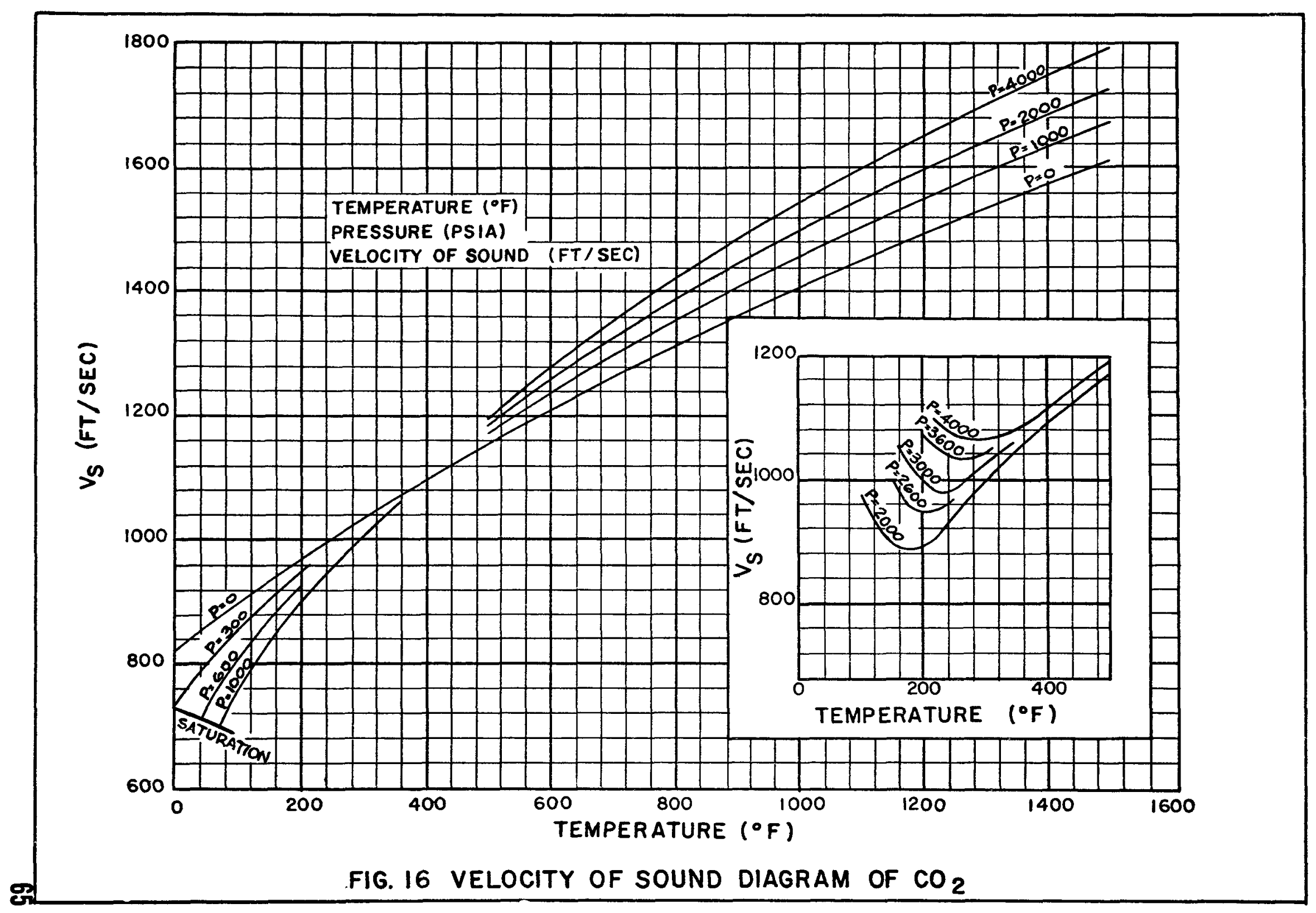




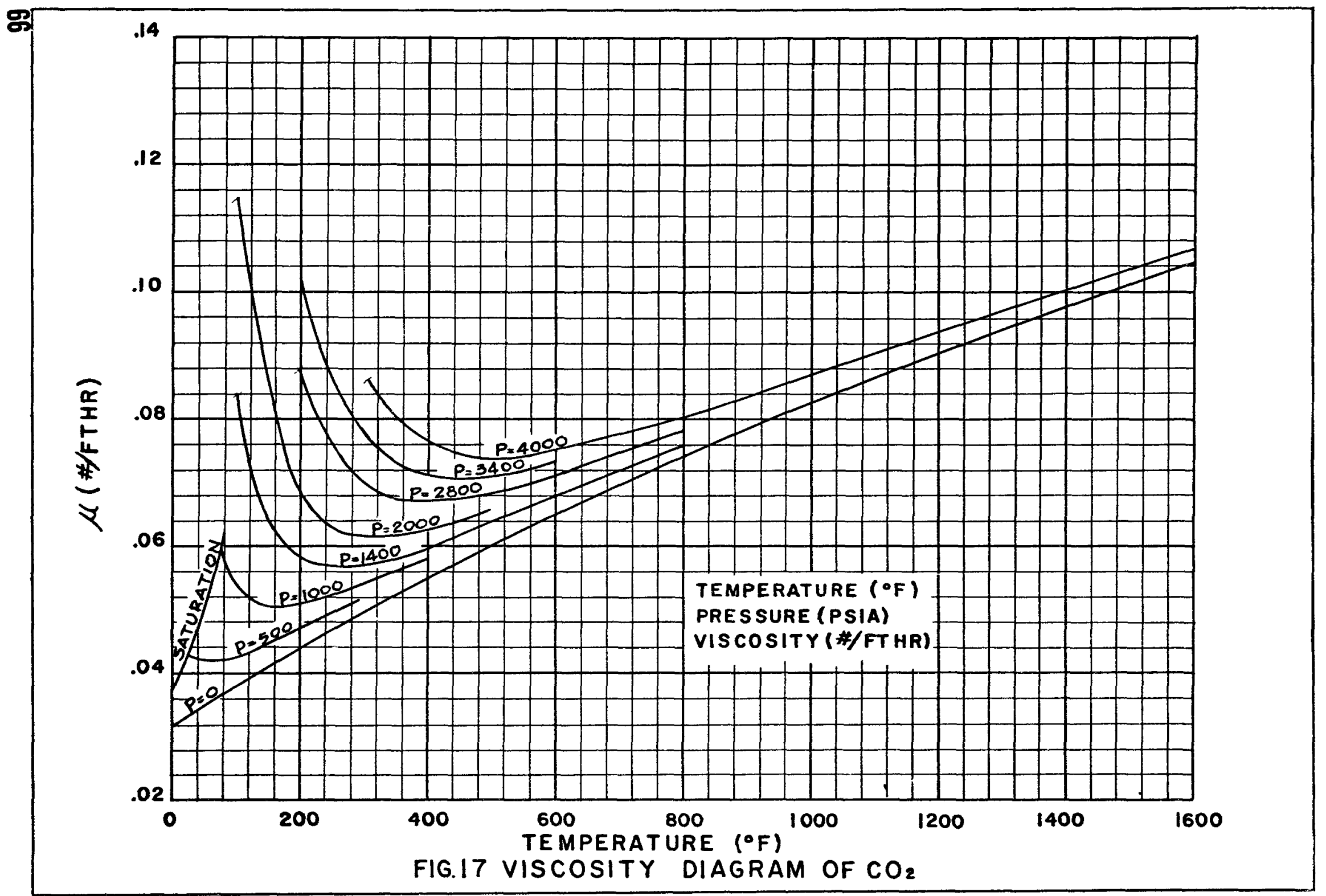




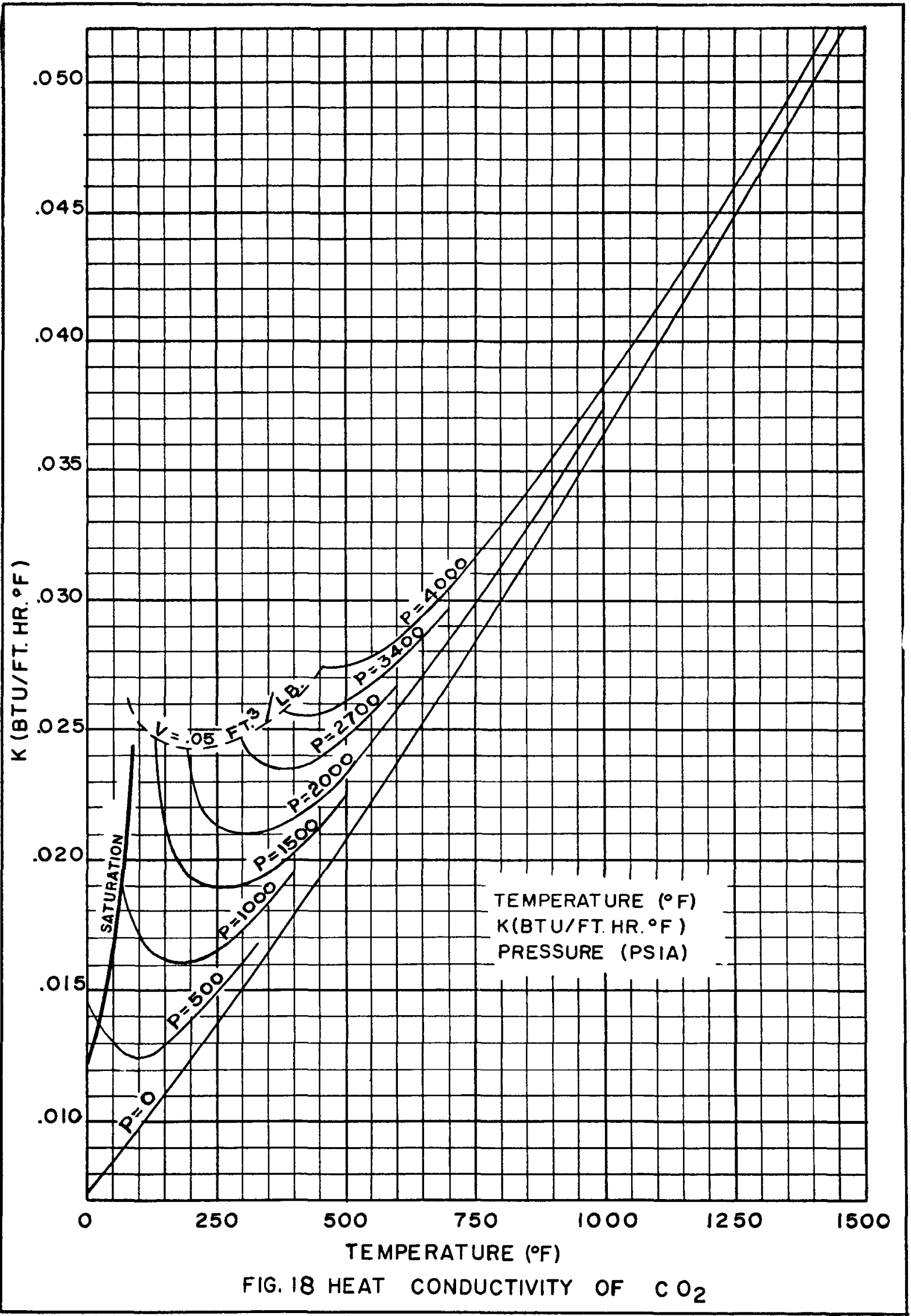




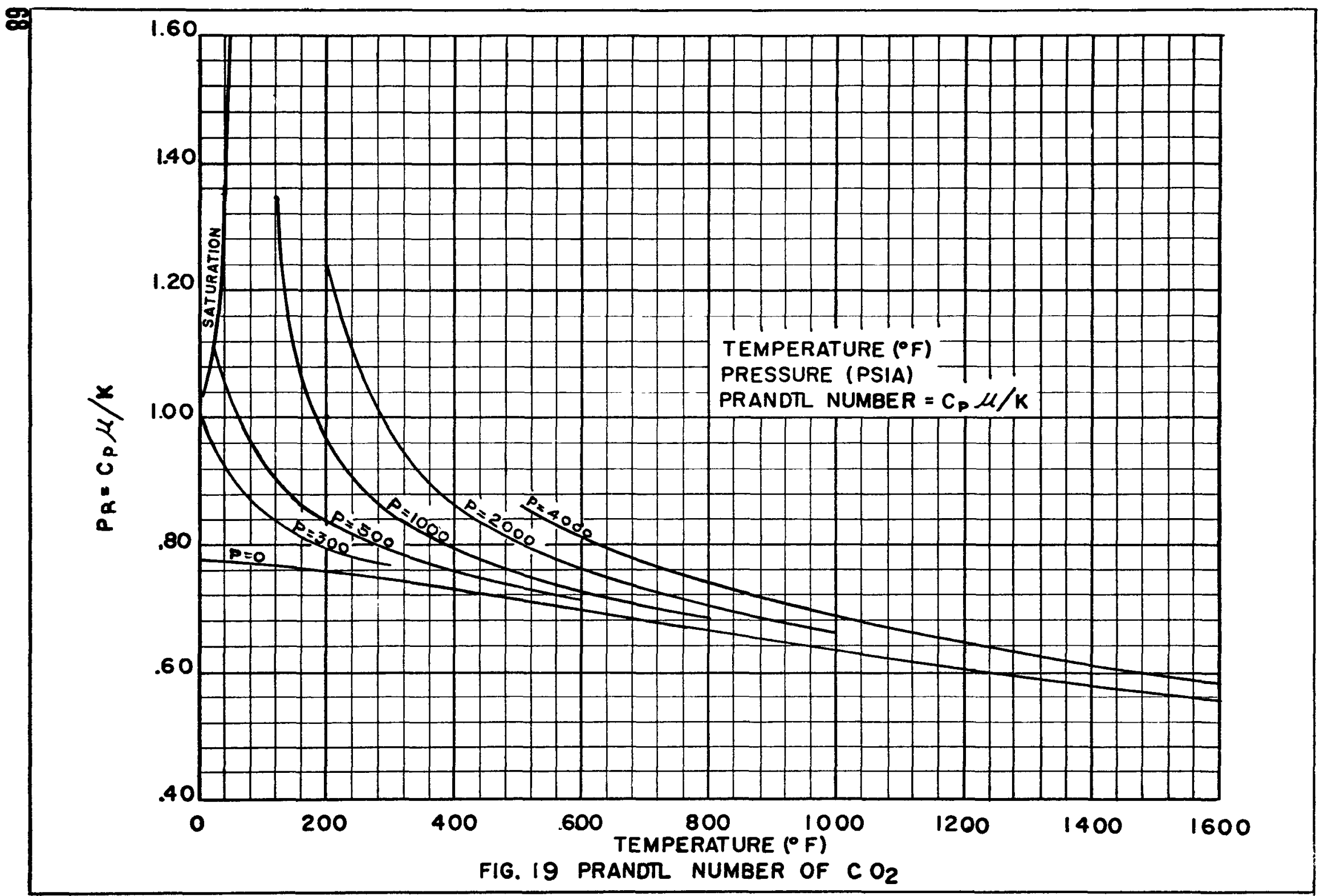




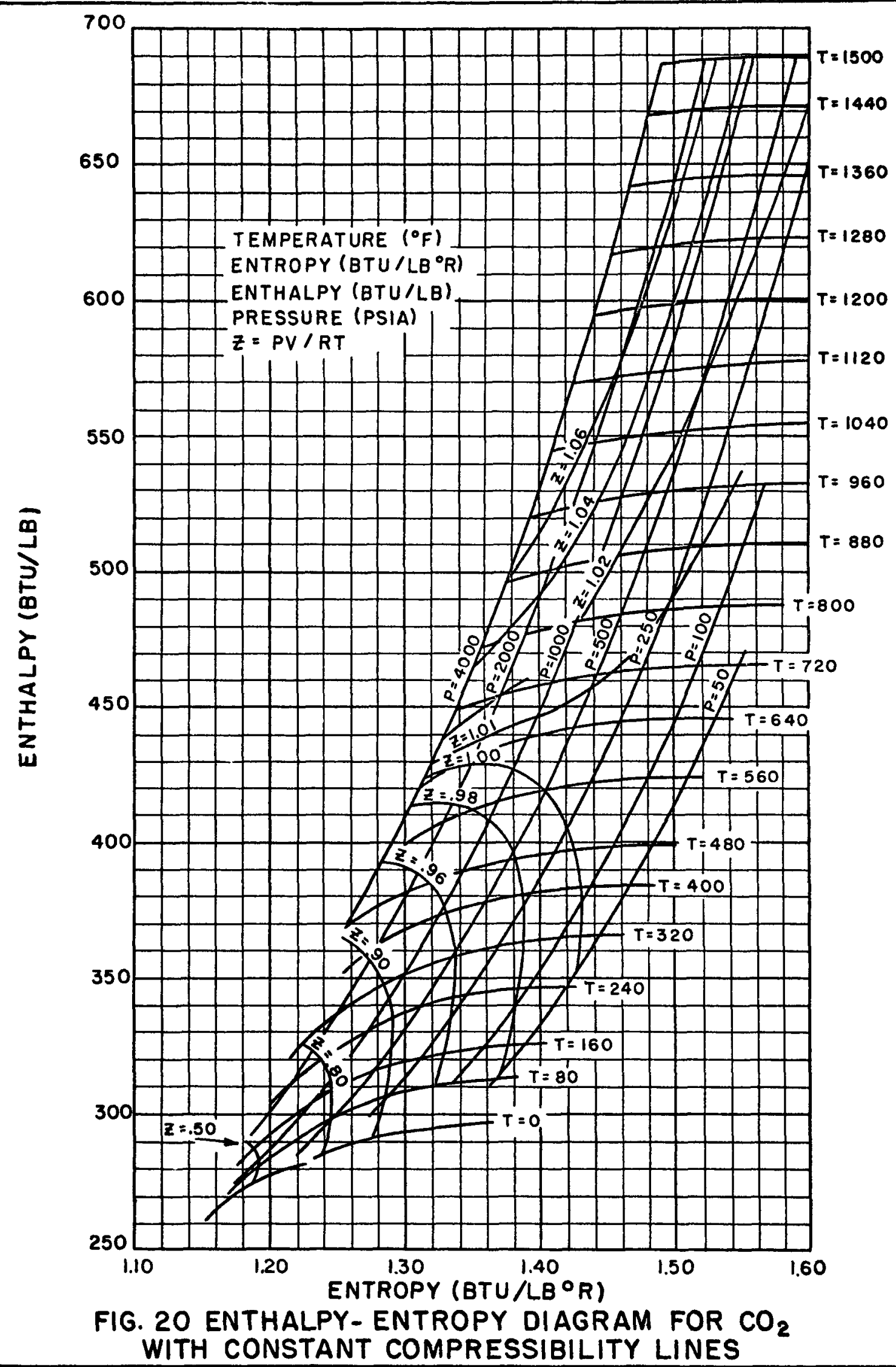




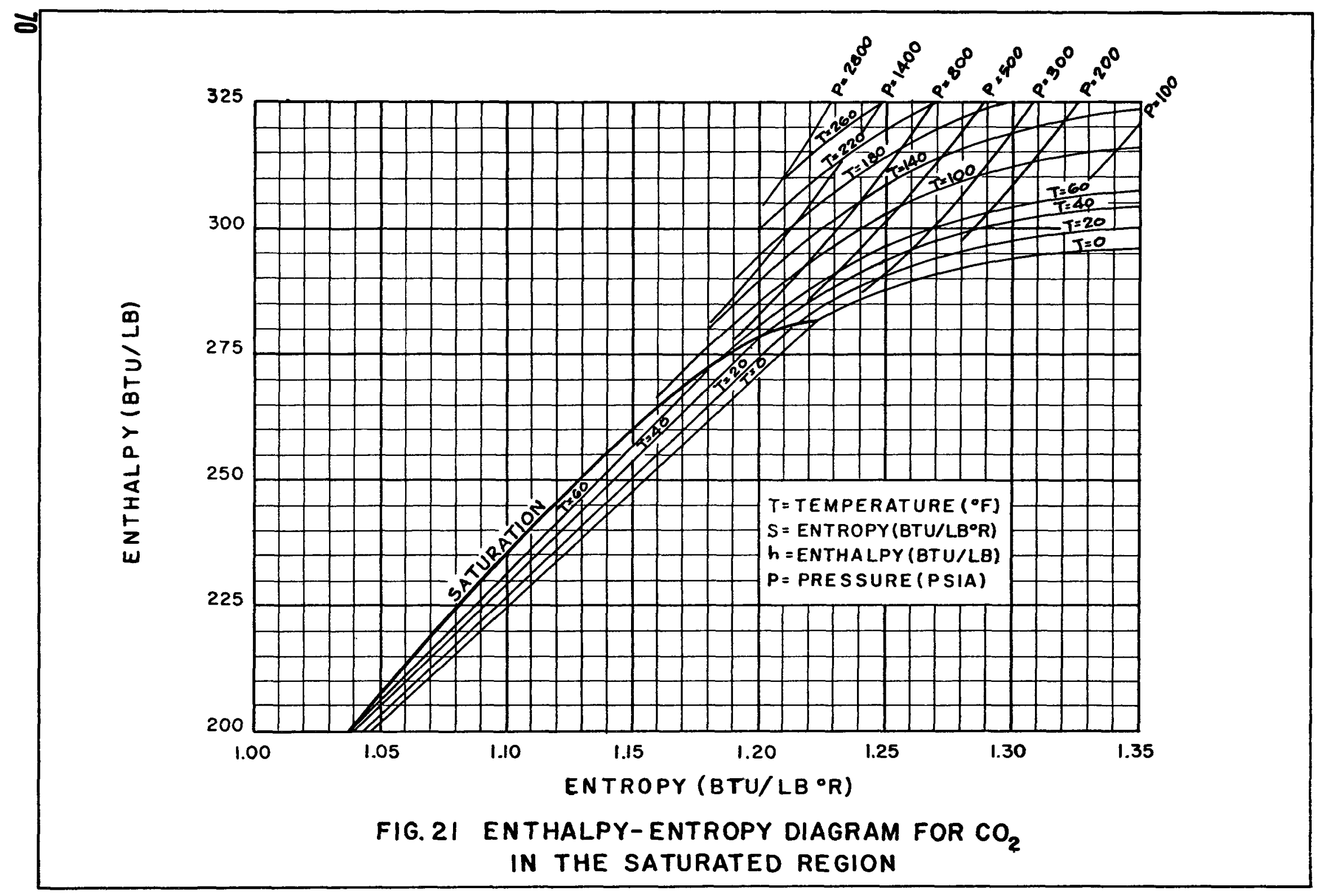




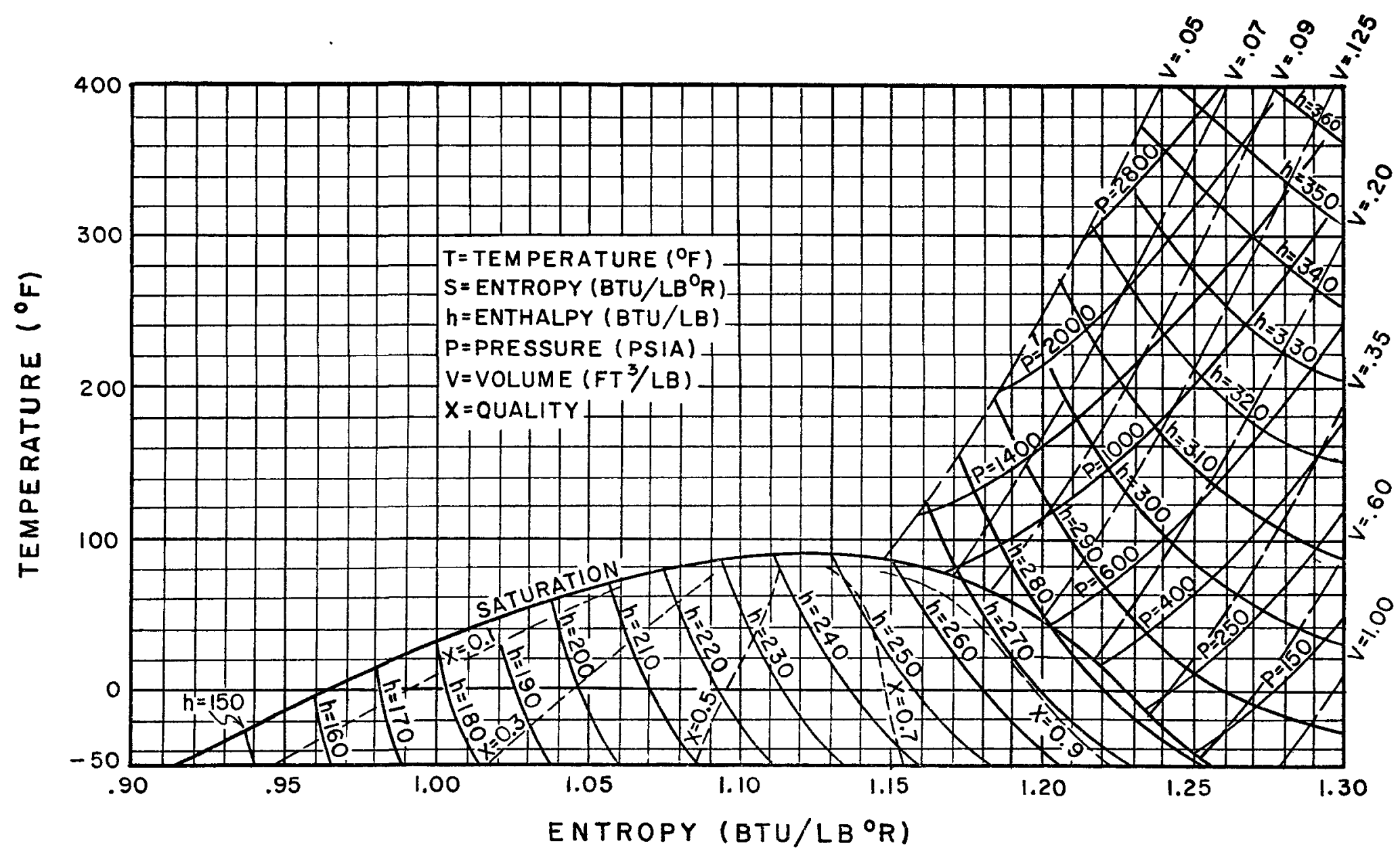

FIG. 22 TEMPERATURE-ENTROPY DIAGRAM FOR $\mathrm{CO}_{2}$ IN THE SATURATED REgION 
-

- 


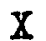

TABLES

Table I

V1scosity Data Taken for Correlation

Data

Method of

Avallable

Reference Experiment Pressure Range

Temperature and

Comings, Mayland, and Egly

$(27)$

transpiration

$104 \rightarrow 221 F$, $1 \rightarrow 2500$ psia

Kiyama and Makita

(31)

rolling ball

$122 \rightarrow 572 \mathrm{~F}$, $1 \rightarrow 1400$ psia

Naldrett and Maass

(48)

oscillatingcritical region disc

Ph1llips

(25)

transpiration

$68 \rightarrow 104 \mathrm{~F}$ $1 \rightarrow 1760$ psia

Schröer and Becker

(49)

rolling ball

$68 \mathrm{~F}$ $1 \rightarrow 1470$ psia

Stakelbeck

(26)

falling ball

$5 \rightarrow 104 \mathrm{~F}$, $1 \rightarrow 1470$ psia 
Table 2

\section{Comparison of Viscosity Data} at $75 \mathrm{C}$ (167F)

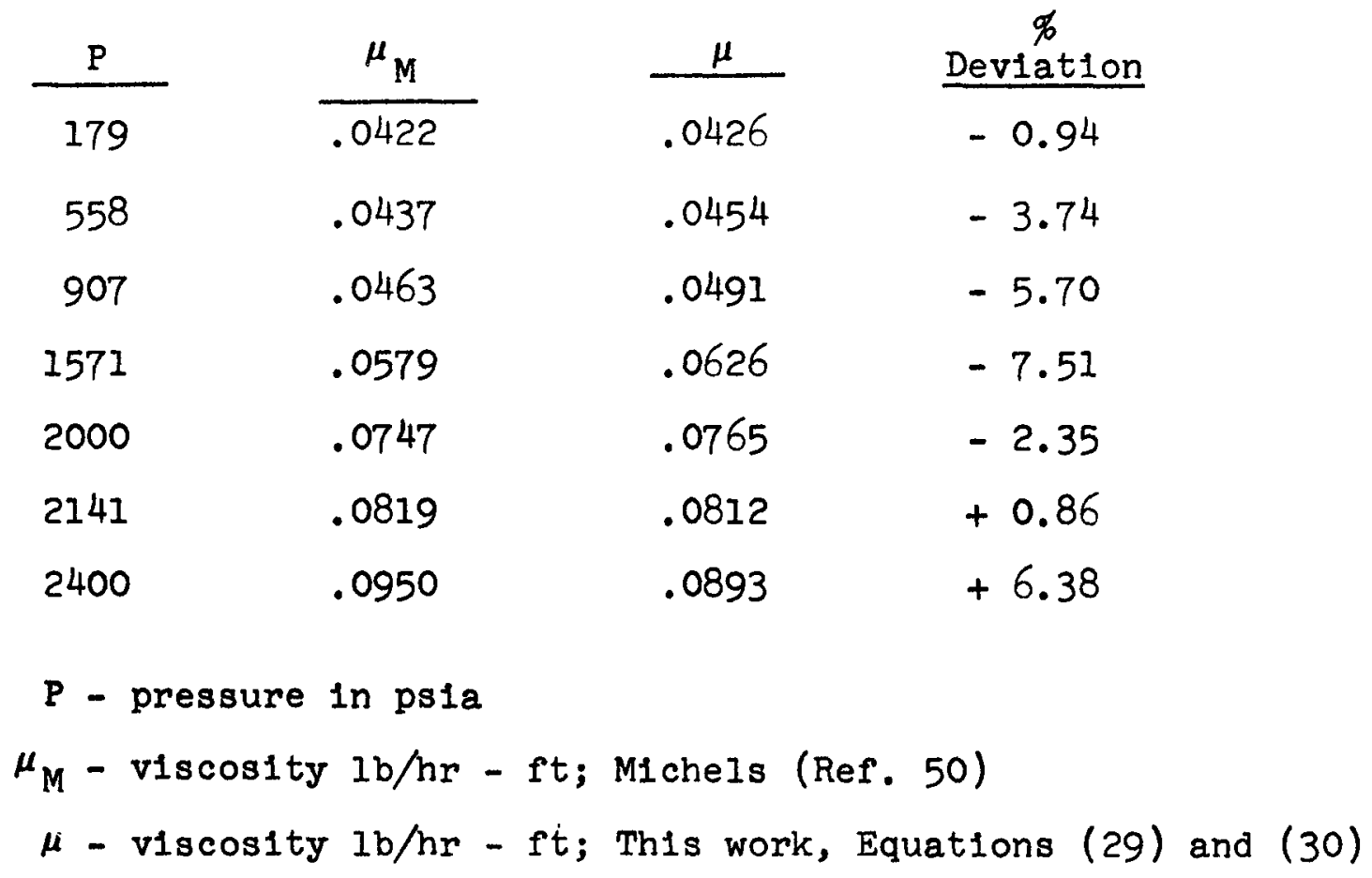


Table 3

Comparison of Heat Conductivity Data

\begin{tabular}{|c|c|c|c|c|}
\hline $\mathrm{P}$ & $t$ & $\mathrm{~K}_{\mathrm{s}}$ & $\mathrm{K}$ & Deviation \\
\hline 20 & 98.6 & .01035 & .01112 & -6.92 \\
\hline 30 & 98.6 & .01062 & .01189 & -10.68 \\
\hline \multirow[t]{3}{*}{100} & 127.9 & .02383 & . C2 478 & -3.83 \\
\hline & 186.8 & .01805 & .01930 & -6.48 \\
\hline & 338.0 & .01877 & .01937 & -3.10 \\
\hline \multirow[t]{2}{*}{200} & 330.8 & .02642 & .02480 & +6.53 \\
\hline & 381.2 & .02320 & .02424 & -4.29 \\
\hline
\end{tabular}

$P$ - pressure in atmospheres

$t$ - temperature in deg. F

$K_{s}$ - Heat conductivity, Btu/hr. ftoF; Stolyarov et al. (Ref. 51)

$K$ - Heat conductivity, Btu/hr. ft ${ }^{\circ}$; This work, Equations (31) and (32) 
Table 4

Thermodynamic Properties of Gaseous Carbon Dioxide

$\begin{array}{ll}\text { V } & \text { Specific volume, } \mathrm{ft}^{3} / 1 \mathrm{~b} \\ \mathrm{H} & \text { Enthalpy, Btu/lb } \\ \mathrm{S} & \text { Entropy, Btu/lb, deg. F } \\ \text { Temperature } & \text { Deg. F }\end{array}$


PRESSURE

PSIA

$\begin{array}{ll}14.696 & V \\ H \\ S\end{array}$

16

$\mathbf{V}$
$\mathrm{H}$
$\mathbf{S}$

$20 \quad$

24

28

32

$36 \quad \mathrm{~V}$

$40 \quad V$

$44 \quad 1$

48

$52 \quad \begin{aligned} & V \\ & H \\ & \end{aligned}$
0

20

40

7.5595 297.61

$2 \cdot 3888$

7.8965

301.60

1.3973

6.9383

297.56

7.2482

301.55

1.3934

5.5373

297.41

1.3747

5.7871

301.42

1.3832

4. 6037

297.27

4.8123

301.28

1.3748

3.9368

297.12

1.3592

$4 \cdot 1164$

301.15

1.3677

3.4366

296.97

1.3529

3.5944

301.01

1. 3615

3.0475

296.82

3.1885

300.87

1. 3561

2.7362

296.68

2.8637

300.74

1.3511

2.4815

296.53

1.3380

2.2692

296.38

1.3339

2.0895

296.22

1.3301
2.5979

300.60

1.3467

2.3764

300.46

1.3426

2.1890

300.32

1.3388
8.2329

305.61

1.4054

7.5576

305.57

1.4016

6.0355

305.44

1.3914

5.0203

305.31

1.3830

4.2954

305.19

1.3760

3.7517

305.06

I. 3698

3.3288

304.94

1.3643

2.9905

304.81

1.3594

2.7137

304.68

1.3550

2.4830

304.55

1.3509

$2 \cdot 2878$

304.43

I. 3471

\section{TEMPERATURE}

$60 \quad 80$

8.5689

309.64

1.4134

8.9044

313.72

1.4210

7.8665

309.61

8.1751

313.68

1.4172

6.2835

309.49

1.3993

6.5311

313.57

1.4070

5.2282

309.38

5.4352

313.46

$1 \cdot 3987$

4.4739

309.26

1.3839

4.6524

313.36

1.3917

3.9085

309.14

1.3778

4.0648

313.24

1.3855

3.4687

309.02

3.6081

313.13

1.3801

3.1168

3.2427

313.02

1.3675

1.3752

2.8289

308.78

1.3630

2.5890

308.67

1.3590

2.9438

312.91

1. 3708

2.6946

312.80

1.3668

2.3860

308.55

2.4838

312.69
1.3630
100

9.2396

317.83

1.4285

8.4832

317.79

1.4247

6.7784

317.69

1.4145

5.6418

317.59

1.4062

4.8300

317.49

1.3992

4.2211

317.39

1.3931

3.7471

317.28

1.3876

3.3682

317.18

$1 \cdot 3828$

3.0582

317.08

1.3784

2.7999

316.97

1.3743

2.5813

316.87
1.3706
120

9.5745

321.98
1.4358

8.7911

321.95

1.4319

7.0253

321.85

1.4218

5.8481

321.75

1.4135

5.0073

321.66

1.4065

4.3767

321.56

1.4004

3.8862

321.47

1.3950

3.4934

321.37

1.3901

3.1723

321.27

1.3857

2.9047

321.18

1.3817

2.6783

321.08
1.3780
140

$9.9091 \quad 10.2435$

$326.17 \quad 330.41$

$1.4429 \quad 1.4498$

$9.0987 \quad 9.4060$

$326.14 \quad 330.38$

$1.4390 \quad 2.4460$

$7.2720 \quad 7.5184$

$326.05 \quad 330.30$

1.42891 .4359

$6.0542 \quad 6.2600$

$325.96 \quad 330.21$

$1.4206 \quad 1.4276$

$5.1843 \quad 5.3611$

325.87

$1.4136 \quad 1.4206$

$4.5320 \quad 4.6870$

$325.78 \quad 330.04$

$1.4075 \quad 1.4145$

$4.0245 \quad 4.1627$

$325.69 \quad 329.96$

$1.4021 \quad 1.4091$

$3.6186 \quad 3.7432$

$325.60 \quad 329.87$

1.39731 .4043

$3.2861 \quad 3.4000$

325.51

$1.3929 \quad 1.3999$

$3.0093 \quad 3.1137$

32504

1. 3889

329.70
1.3959

$2.7751 \quad 2.8717$

$325 \cdot 32$

1. 3852

329.61 


\begin{tabular}{|c|c|c|c|c|c|c|c|c|c|}
\hline 56 & $\begin{array}{l}1.9355 \\
296.07 \\
1.3265\end{array}$ & $\begin{array}{l}2.0283 \\
300.18 \\
1.3353\end{array}$ & $\begin{array}{l}2.1204 \\
304.30 \\
1.3436\end{array}$ & $\begin{array}{l}2.2120 \\
308.43 \\
1.3517\end{array}$ & $\begin{array}{l}2.3031 \\
312.58 \\
1.3596\end{array}$ & $\begin{array}{l}2.3939 \\
316.76 \\
1.3672\end{array}$ & $\begin{array}{l}2.4843 \\
320.98 \\
1.3746\end{array}$ & $\begin{array}{l}2.5744 \\
325.23 \\
1.3818\end{array}$ & $\begin{array}{l}2.6642 \\
329.53 \\
1.3888\end{array}$ \\
\hline 60 & $\begin{array}{l}1.8020 \\
295.92 \\
1.3232\end{array}$ & $\begin{array}{l}1.8890 \\
300.04 \\
1.3320\end{array}$ & $\begin{array}{l}1.9754 \\
304.17 \\
1.3404\end{array}$ & $\begin{array}{l}2.0612 \\
308.31 \\
1.3485\end{array}$ & $\begin{array}{l}2.1465 \\
312.47 \\
1.3563\end{array}$ & $\begin{array}{l}2.2315 \\
316.66 \\
1.3639\end{array}$ & $\begin{array}{l}2.3161 \\
320.88 \\
1.3714\end{array}$ & $\begin{array}{l}2.4004 \\
325.14 \\
1.3786\end{array}$ & $\begin{array}{l}2.4844 \\
329.44 \\
1.3856\end{array}$ \\
\hline 70 & $\begin{array}{l}1.5350 \\
295.54 \\
1.3157\end{array}$ & $\begin{array}{l}1.6105 \\
299.69 \\
1.3245\end{array}$ & $\begin{array}{l}1.6853 \\
303.84 \\
1.3330\end{array}$ & $\begin{array}{l}1.7595 \\
308.01 \\
1.3412\end{array}$ & $\begin{array}{l}1.8333 \\
312.19 \\
1.3491\end{array}$ & $\begin{array}{l}1.9066 \\
316.40 \\
1.3567\end{array}$ & $\begin{array}{l}1.9797 \\
320.64 \\
1.3641\end{array}$ & $\begin{array}{l}2.0524 \\
324.91 \\
1.3714\end{array}$ & $\begin{array}{l}2.1248 \\
329.22 \\
1.3784\end{array}$ \\
\hline 80 & $\begin{array}{l}1.3346 \\
295.15 \\
1.3091\end{array}$ & $\begin{array}{l}1.4015 \\
299.33 \\
1.3180\end{array}$ & $\begin{array}{l}1.4676 \\
303.52 \\
1.3266\end{array}$ & $\begin{array}{l}1.5332 \\
307.70 \\
1.3348\end{array}$ & $\begin{array}{l}1.5983 \\
311.91 \\
1.3427\end{array}$ & $\begin{array}{l}1.6530 \\
316.14 \\
1.3504\end{array}$ & $\begin{array}{l}1.7273 \\
320.39 \\
1.3578\end{array}$ & $\begin{array}{l}1.7914 \\
324.68 \\
1.3651\end{array}$ & $\begin{array}{l}1.3551 \\
329.01 \\
1.3722\end{array}$ \\
\hline 90 & $\begin{array}{l}1.1787 \\
294.75 \\
1.3033\end{array}$ & $\begin{array}{l}1.2388 \\
298.98 \\
1.3122\end{array}$ & $\begin{array}{l}1.2983 \\
303.19 \\
1.3208\end{array}$ & $\begin{array}{l}1.3572 \\
307.40 \\
1.3291\end{array}$ & $\begin{array}{l}1.4155 \\
311.63 \\
1.3371\end{array}$ & $\begin{array}{l}1.4735 \\
315.87 \\
1.3448\end{array}$ & $\begin{array}{l}1.5311 \\
320.15 \\
1.3523\end{array}$ & $\begin{array}{l}1.5883 \\
324.45 \\
1.3596\end{array}$ & $\begin{array}{l}1.6454 \\
328.79 \\
1.3667\end{array}$ \\
\hline 100 & $\begin{array}{l}1.0538 \\
294.36 \\
1.2979\end{array}$ & $\begin{array}{l}1.1087 \\
298.61 \\
1.3070\end{array}$ & $\begin{array}{l}1.1628 \\
302.85 \\
1.3156\end{array}$ & $\begin{array}{l}1.2163 \\
307.09 \\
1.3239\end{array}$ & $\begin{array}{l}1.2693 \\
311.34 \\
1.3320\end{array}$ & $\begin{array}{l}1.3218 \\
315.61 \\
1.3397\end{array}$ & $\begin{array}{l}1.3740 \\
319.90 \\
1.3472\end{array}$ & $\begin{array}{l}1.4259 \\
324.22 \\
1.3546\end{array}$ & $\begin{array}{l}1.4775 \\
328.57 \\
1.3517\end{array}$ \\
\hline 110 & $\begin{array}{l}0.9516 \\
293.95 \\
1.2931\end{array}$ & $\begin{array}{l}1.0021 \\
298.24 \\
1.3022\end{array}$ & $\begin{array}{l}1.0519 \\
302.52 \\
1.3109\end{array}$ & $\begin{array}{l}1.1010 \\
306.78 \\
1.3193\end{array}$ & $\begin{array}{l}1.1496 \\
311.05 \\
1.3273\end{array}$ & $\begin{array}{l}1.1978 \\
315.34 \\
1.3351\end{array}$ & $\begin{array}{l}1.2455 \\
319.65 \\
1.3427\end{array}$ & $\begin{array}{l}1.2930 \\
323.99 \\
1.3500\end{array}$ & $\begin{array}{l}1.3402 \\
328.35 \\
1.3572\end{array}$ \\
\hline 120 & $\begin{array}{l}0.8664 \\
293.54 \\
1.2885\end{array}$ & $\begin{array}{l}0.9133 \\
297.87 \\
1.2977\end{array}$ & $\begin{array}{l}0.9594 \\
302.18 \\
1.3065\end{array}$ & $\begin{array}{l}1.0049 \\
306.47 \\
1.3149\end{array}$ & $\begin{array}{l}1.0498 \\
310.77 \\
1.3230\end{array}$ & $\begin{array}{l}1.0943 \\
315.07 \\
1.3309\end{array}$ & $\begin{array}{l}1.1385 \\
319.40 \\
1.3384\end{array}$ & $\begin{array}{l}1.1823 \\
323.75 \\
1.3458\end{array}$ & $\begin{array}{l}1.2258 \\
328.13 \\
1.3530\end{array}$ \\
\hline 130 & $\begin{array}{l}0.7942 \\
293.13 \\
1.2843\end{array}$ & $\begin{array}{l}0.8381 \\
297.50 \\
1.2936\end{array}$ & $\begin{array}{l}0.8812 \\
301.83 \\
1.3025\end{array}$ & $\begin{array}{l}0.9236 \\
306.16 \\
1.3109\end{array}$ & $\begin{array}{r}0.9654 \\
310.48 \\
1.3191\end{array}$ & $\begin{array}{l}1.0068 \\
314.80 \\
1.3269\end{array}$ & $\begin{array}{l}1.0478 \\
319.15 \\
1.3345\end{array}$ & $\begin{array}{l}1.0885 \\
323.52 \\
1.3419\end{array}$ & $\begin{array}{l}1.1289 \\
327.91 \\
1.3491\end{array}$ \\
\hline 140 & $\begin{array}{l}0.7322 \\
292.71 \\
1.2804\end{array}$ & $\begin{array}{l}0.7736 \\
297.12 \\
1.2897\end{array}$ & $\begin{array}{l}0.8140 \\
301.49 \\
1.2986\end{array}$ & $\begin{array}{l}0.8538 \\
305.84 \\
1.3072\end{array}$ & $\begin{array}{l}0.8930 \\
310.18 \\
1.3154\end{array}$ & $\begin{array}{l}0.9318 \\
314.53 \\
1.3233\end{array}$ & $\begin{array}{l}0.9701 \\
318.90 \\
1.3309\end{array}$ & $\begin{array}{l}1.0082 \\
323.28 \\
1.3383\end{array}$ & $\begin{array}{l}1.0459 \\
327.69 \\
1.3456\end{array}$ \\
\hline 150 & $\begin{array}{l}0.6785 \\
292.28 \\
1.2766\end{array}$ & $\begin{array}{l}0.7176 \\
296.73 \\
1.2861\end{array}$ & $\begin{array}{l}0.7558 \\
301.14 \\
1.2951\end{array}$ & $\begin{array}{l}0.7933 \\
305.52 \\
1.3036\end{array}$ & $\begin{array}{l}0.8303 \\
309.89 \\
1.3119\end{array}$ & $\begin{array}{l}0.8667 \\
314.26 \\
1.3198\end{array}$ & $\begin{array}{l}0.9028 \\
318.64 \\
1.3275\end{array}$ & $\begin{array}{l}0.9385 \\
323.05 \\
1.3350\end{array}$ & $\begin{array}{l}0.9740 \\
327.47 \\
1.3422\end{array}$ \\
\hline
\end{tabular}


PRESSURE

PSIA

0

20

40

160

0.6314

291.85

1.2731

170

0.5898

291.41

1.2697

0.5528

$180 \quad \mathrm{~V}$
$\mathrm{H}$
$\mathrm{S}$

190

200

220

240

260

280

300

320

290.96

1.2664

0.6686

296.35

1.2826

0.7048

300.79

1.2917

0.6253

295.95

1.2793

0.6598

300.43

1. 2884

0.5867

295.55

1.2762

0.6198

300.07

I. 2854

0.5196

290.51

0.5523

295.15

0.5840

299.71

1. 2824

0.4896

1.2731

0.5517

0.5212
294.74

294.74
1.2702

299.34

1.2603

0.4674

289.11

1.2545

293.91

1.2647

0.4959

298.60

1. 2743

0.3943

288.12

0.4224

293.05

0.4492

1.2491

1.2595

297.83

0.3572
287.10

0.3841

292.17

0.4096

1.2439

1. 2546

297.05

0.3512

0.3756

296.25

286.04

1.25

1. 2601

0.2973

0.3225

284.93

290.31

0.3460

295.43

1. 2558

0.2973

289.33

0.3201

294.58

1. 2517

1.2410

\section{TEMPERATURE}

60

80

0.7404

305.20

1.3003

0.7753

309.59

I. 3086

0.6937

3048

1. 2971

0.6521

304.54

1.2941

0.6149

304.21

1.2913

0.5814

303.88

1.2885

0.5234

303.20

I. 2833

0.4751

302.52

0.7269

02

1.3055

0.6837

308.99

1.3025

0.6452

308.69

1. 2997

0.6104

308.39

1.2970

0.5504

307.77

1.2919

0.5003

307.14

1. 2872

0.4342

301.82

0.4578

306.51

1.2827

0.3989

301.10

0.4214

305.86

1.2786

1.2696

0.3684

300.37

1. 2655

0.3898

305.21

1.2746

0.3415

299.63

0.3621

304.54

1.2708
100

120

140

160

0.8098

313.99

1.3166

0.8439

318.39

1. 3243

0.8776

322.81

1.3318

0.9110

0.7596

31

0.7919

0.8238

22

1.3288

I. 3213

0.7149

313.43

0.7456

317.88

0.7760

322.33

1.3259

0.6749

313.15

0.7042

317.62

0.7332

322.09

1.3232

0.6389

312.87

0.6670

317.36

0.6947

321.85

1.3206

$0.5767 \quad 0.6026$

312.31

1.3002

316.83
1.3081

0.6281

321.36

1.3158

0.5248

311.73

0.5490

316.30

0.5727

320.87

1.3113

0.4809

$311 \cdot 15$

1.2912

0.5035

315.77

0.5257

320.38

1.3071

$0.4432 \quad 0.4645$

$310.56 \quad 315.23$

1.2871

1.2953

319.88

1. 3031

0.4307

0.4105

309.97

314.68

0.4505

319.37

1. 2994

0.3819

309.37

. .2796

0.4011

314.13

0.4200

318.86

1. 2959
327.25

1. 3391

0.8554

327.03

1. 3361

0.8060

326.80

1.3333

0.7615

326.58

1.3306

0.7221

$326 \cdot 35$

1. 3280

0.6533

325.90

I. 3232

0.5961

325.44

1. 3188

0.5476

$324 \cdot 98$

1.3146

0.5060

324.52

1.3107

0.4699

324.05

1.3071

0.4384

323.58

1. 3037 
0.2642

305.55

1.2503

$1.2: 03$

1.2507

0.2505

0.1989

287.85

0.2175

0.2346

293.89

299.52

304.88

1.2574

0.1869

286.73

0.2055

292.98

0.2223

298.74

0.2378

304.20

1. 2447

1.2546

0.1943

0.2109

0.1757

285.55

292.03

297.94

0.2262

303.51

1.2417

1.2518

0.2003

0.1651

0.1839

297.13

284.31

291.06

0.2154

302.80

1.2491

0.1552

282.99

0.1741

0.1905

290.05

1.2242

296.29

1.2359

0.2054

302.09

1. 2465

\begin{abstract}
120
\end{abstract}
0.3750

313.57

1.2845

\subsection{8}

313.01

1.2812

0.3310

312.44

1. 2781

0.3122

311.86

1. 2750

0.2952

311.28

1.2721

0.2797

310.68

1.2593

0.2656

310.08

I. 2665

0.2526

309.48

1.2638

0.2406

308.86

1.2612

0.2296

308.24

1.2586

0.2193

307.61

1.2561
140

0.3930

318.35

1.2926

0.3590

317.83

1.2894

0.3475

317.31

1. 2863

0.3231

316.78

I. 2934

0.3106

316.25

1.2805

0.2947

0.2947

1. 2775

0.2301

315.17

1.2751

0.2667

3:4.62

1. 2725

0.2544

314.07

i. 2700

0.2430

313.51

I. 2576

0.2325

312.94

1.2652
160

0.4105

323.11

1. 3004

0.3358

322.53

1.2972

0.3636

322.15

1. 2942

0.3435

321.66

l. 2914

0.3255

321.17

1.2836

0.3091

320.58

1. 2859

0.2941

320.19

1.2833

0.2504

319.69

i. 2808

0.2677

319.18

1.2784

$0.25 \in 0$

318.67

1.2760

0.2451

318.16

1.2737 
PRESSURE

PSIA

560

$V$
$H$
$S$

580

$600 \quad \mathrm{H}$

$620 \quad \mathrm{H}$

$640 \quad \begin{aligned} & \mathrm{H} \\ & \mathrm{H}\end{aligned}$

$660 \quad \mathrm{~V}$

$680 \mathrm{~V}$

$\mathrm{S}$

700

$720 \quad \mathrm{~V}$

720

740

$\infty \begin{array}{lll} & & \\ & 760 & H \\ S\end{array}$
0

20

40

0.1458

281.58

1.2064

1

289.00
1.2210

0.1564

287.91

1.2177

0.1483

286.76

1.2145

0.1406

285.56

1.2112

0.1332

284.30

1.2078

0.1262

282.96

1. 2043

0.1194

281.54

1.2007

0.2128

280.01

1.1969

0.1063

278.35

1.1930

0.1000

276.53

1.1888

$$
\begin{aligned}
& 286.42 \\
& 1.2074
\end{aligned}
$$

285.21

1.2044
0.1137

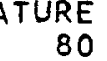

0.1814
295.44

1.2331

0.1728
294.56

1.2303

0.1647
293.65

1.2274

0.1572

292.72

1.2246

0.1500

0.16
1.2218

0.1432

290.77

0.1367

289.74

0.1306

288.67

1.2133

0.1247

287.57

1.2103

1

294.94

1.2237

0.2338

294.05

1.2212

0.2285

293.14

1.2188
120

0.2098

306.97

1.2537

0.2009

306.32

1. 2512

0.1925

305.66

1.2489

0.1847

304.99

1. 2465

0.1774

304.31

1.2442

0.1705

303.62

1.2419

0.1640

302.92

1.2397

0.1578

302.21

1.2374

0.1520

301.48

1.2352

0.1464

300.75

1.2330

0.1412

300.00

1.2308
140

0.2227

312.37

1. 2628

0.2135

311.79

1.2605

0.2050

311.21

1.2583

0.1970

310.62

1.2560

0.1895

310.02

1. 2539

0.1824

309.42

1.2517

0.1758

308.80

1.2496

0.1695

308.18

308.18
1.2475

0.1635

307.56

1.2455

0.1579

306.92

1. 2434

0.1525

306.28

1.2414
160

0.2350

317.64

1.2714

0.2256

317.12

1.2692

0.2169

316.59

1.2671

0.2086

316.06

1.2650

0.2009

315.53

1.2629

0.1937

314.99

1.2608

0.1869

314.44

1.2588

0.1804

313.89

1. 2569

0.1743

313.34

I. 2549

0.1685

312.78

1.2530

0.1631

312.21

1.2511 


PRESSURE
PSIA

0

40

TEMPERATURE

$60 \quad 80$

\begin{tabular}{|c|c|c|c|c|}
\hline 80 & 100 & 120 & 140 & 160 \\
\hline $\begin{array}{l}0.1085 \\
283.95 \\
1.2013\end{array}$ & $\begin{array}{l}0.1235 \\
292.20 \\
1.2163\end{array}$ & $\begin{array}{l}0.1361 \\
299.23 \\
1.2286\end{array}$ & $\begin{array}{l}0.1474 \\
305.63 \\
1.2394\end{array}$ & $\begin{array}{l}0.1579 \\
311.64 \\
1.2492\end{array}$ \\
\hline $\begin{array}{l}0.1034 \\
282.63 \\
1.1981\end{array}$ & $\begin{array}{l}0.1187 \\
291.24 \\
1.2138\end{array}$ & $\begin{array}{l}0.1314 \\
298.46 \\
1.2264\end{array}$ & $\begin{array}{l}0.1426 \\
304.97 \\
1.2374\end{array}$ & $\begin{array}{l}0.1529 \\
311.07 \\
1.2474\end{array}$ \\
\hline $\begin{array}{l}0.0986 \\
281.23 \\
1.1949\end{array}$ & $\begin{array}{l}0.1141 \\
290.25 \\
1.2112\end{array}$ & $\begin{array}{l}0.1268 \\
297.67 \\
1.2242\end{array}$ & $\begin{array}{l}0.1380 \\
304.31 \\
1.2355\end{array}$ & $\begin{array}{l}0.1482 \\
310.49 \\
1.2456\end{array}$ \\
\hline $\begin{array}{l}0.0938 \\
279.75 \\
1.1915\end{array}$ & $\begin{array}{l}0.1097 \\
289.23 \\
1.2087\end{array}$ & $\begin{array}{l}0.1224 \\
296.86 \\
1.2221\end{array}$ & $\begin{array}{l}0.1336 \\
303.63 \\
1.2335\end{array}$ & $\begin{array}{l}0.1437 \\
309.90 \\
1.2438\end{array}$ \\
\hline $\begin{array}{l}0.0892 \\
278.17 \\
1.1880\end{array}$ & $\begin{array}{l}0.1055 \\
288.18 \\
1.2061\end{array}$ & $\begin{array}{l}0.1183 \\
296.04 \\
1.2199\end{array}$ & $\begin{array}{l}0.1294 \\
302.95 \\
1.2316\end{array}$ & $\begin{array}{l}0.2395 \\
309.31 \\
1.2420\end{array}$ \\
\hline $\begin{array}{l}0.0846 \\
276.49 \\
1.1843\end{array}$ & $\begin{array}{l}0.1014 \\
287.10 \\
1.2035\end{array}$ & $\begin{array}{l}0.1143 \\
295.21 \\
1.2177\end{array}$ & $\begin{array}{l}0.1254 \\
302.25 \\
1.2296\end{array}$ & $\begin{array}{l}0.1354 \\
308.71 \\
1.2402\end{array}$ \\
\hline $\begin{array}{l}0.0802 \\
274.69 \\
1.1804\end{array}$ & $\begin{array}{l}0.0974 \\
285.98 \\
1.2009\end{array}$ & $\begin{array}{l}0.1104 \\
294.35 \\
1.2155\end{array}$ & $\begin{array}{l}0.1215 \\
301.55 \\
1.2277\end{array}$ & $\begin{array}{l}0.1315 \\
308.11 \\
1.2384\end{array}$ \\
\hline $\begin{array}{l}0.0758 \\
272.75 \\
1.1763\end{array}$ & $\begin{array}{l}0.0936 \\
284.82 \\
1.1982\end{array}$ & $\begin{array}{l}0.1068 \\
293.48 \\
1.2134\end{array}$ & $\begin{array}{l}0.1178 \\
300.84 \\
1.2258\end{array}$ & $\begin{array}{l}0.1277 \\
307.50 \\
1.2367\end{array}$ \\
\hline $\begin{array}{l}0.0715 \\
270.66 \\
1.1719\end{array}$ & $\begin{array}{l}0.0900 \\
283.63 \\
1.1955\end{array}$ & $\begin{array}{l}0.1032 \\
292.60 \\
1.2112\end{array}$ & $\begin{array}{l}0.1143 \\
300.12 \\
1.2239\end{array}$ & $\begin{array}{l}0.1241 \\
306.88 \\
1.2350\end{array}$ \\
\hline \multirow[t]{2}{*}{$\begin{array}{l}0.0673 \\
268.46 \\
1.1674\end{array}$} & $\begin{array}{l}0.0364 \\
282.39 \\
1.1927\end{array}$ & $\begin{array}{l}0.0998 \\
291.69 \\
1.2090\end{array}$ & $\begin{array}{l}0.1109 \\
299.39 \\
1.2220\end{array}$ & $\begin{array}{l}0.1207 \\
306.26 \\
1.2332\end{array}$ \\
\hline & $\begin{array}{l}0.0830 \\
281.11 \\
1.1898\end{array}$ & $\begin{array}{l}0.0965 \\
290.77 \\
1.2068\end{array}$ & $\begin{array}{l}0.1076 \\
298.64 \\
1.2201\end{array}$ & $\begin{array}{l}0.1174 \\
305064 \\
1.2315\end{array}$ \\
\hline
\end{tabular}




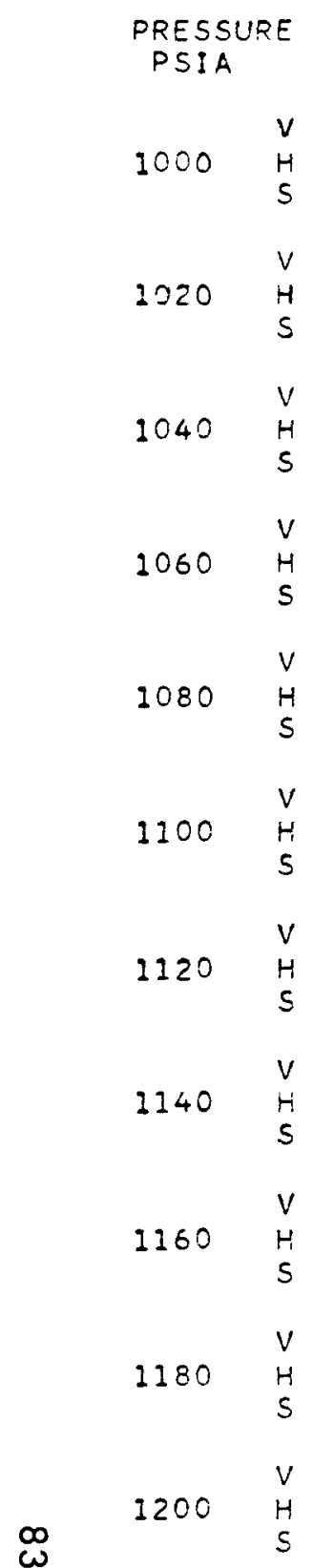

TEMPERA TURE

60 TEMPERATURE

40

80

\begin{tabular}{|c|c|c|c|}
\hline 100 & 120 & 140 & 260 \\
\hline $\begin{array}{l}0.0797 \\
279.79 \\
1.2870\end{array}$ & $\begin{array}{l}0.0934 \\
289.83 \\
1.2045\end{array}$ & $\begin{array}{l}0.1045 \\
207.89 \\
1.2182\end{array}$ & $\begin{array}{l}0.1142 \\
305.00 \\
1.2298\end{array}$ \\
\hline $\begin{array}{l}0.0765 \\
278.43 \\
1.1840\end{array}$ & $\begin{array}{l}0.0903 \\
283.87 \\
1.2023\end{array}$ & $\begin{array}{l}0.1014 \\
297.13 \\
1.2163\end{array}$ & $\begin{array}{l}0.11111 \\
304.37 \\
1.2281\end{array}$ \\
\hline $\begin{array}{l}0.0734 \\
277.02 \\
1.1810\end{array}$ & $\begin{array}{l}0.0874 \\
287.89 \\
1.2001\end{array}$ & $\begin{array}{l}0.0985 \\
296.36 \\
1.2144\end{array}$ & $\begin{array}{l}0.1032 \\
303.72 \\
1.2264\end{array}$ \\
\hline $\begin{array}{l}0.0704 \\
275.58 \\
1.1780\end{array}$ & $\begin{array}{l}0.0845 \\
286.89 \\
1.1978\end{array}$ & $\begin{array}{l}0.0957 \\
295.58 \\
1.2125\end{array}$ & $\begin{array}{l}0.1053 \\
303.07 \\
1.2248\end{array}$ \\
\hline $\begin{array}{l}0.0675 \\
274.11 \\
1.1749\end{array}$ & $\begin{array}{l}0.0818 \\
285.88 \\
1.1955\end{array}$ & $\begin{array}{l}0.0930 \\
294.79 \\
2.2106\end{array}$ & $\begin{array}{l}0.1026 \\
302.42 \\
1.2231\end{array}$ \\
\hline $\begin{array}{l}0.0648 \\
272.62 \\
1.1719\end{array}$ & $\begin{array}{l}0.0792 \\
284.84 \\
1.1033\end{array}$ & $\begin{array}{l}0.0904 \\
293.99 \\
1.2087\end{array}$ & $\begin{array}{l}0.1000 \\
301.76 \\
1.2214\end{array}$ \\
\hline $\begin{array}{l}0.0623 \\
271.13 \\
1.1688\end{array}$ & $\begin{array}{l}0.0766 \\
283.80 \\
1.1910\end{array}$ & $\begin{array}{l}0.0879 \\
293.18 \\
1.2068\end{array}$ & $\begin{array}{l}0.0575 \\
301.09 \\
1.2198\end{array}$ \\
\hline $\begin{array}{l}0.0598 \\
269.65 \\
1.1658\end{array}$ & $\begin{array}{l}0.0742 \\
282.73 \\
1.1887\end{array}$ & $\begin{array}{l}0.0855 \\
292.37 \\
1.2050\end{array}$ & $\begin{array}{l}0.0950 \\
300.42 \\
1.2181\end{array}$ \\
\hline $\begin{array}{l}0.0576 \\
268.21 \\
1.1628\end{array}$ & $\begin{array}{l}0.0718 \\
281.66 \\
1.1864\end{array}$ & $\begin{array}{l}0.0831 \\
291.54 \\
1.2031\end{array}$ & $\begin{array}{l}0.0926 \\
295.74 \\
1.2165\end{array}$ \\
\hline $\begin{array}{l}0.0556 \\
266.82 \\
1.1600\end{array}$ & $\begin{array}{l}0.0696 \\
280.57 \\
1.1840\end{array}$ & $\begin{array}{l}0.0808 \\
200.71 \\
2.2012\end{array}$ & $\begin{array}{l}0.0904 \\
299.05 \\
1.2148\end{array}$ \\
\hline $\begin{array}{l}0.0537 \\
265.49 \\
1.7572\end{array}$ & $\begin{array}{l}0.0574 \\
279.49 \\
1.11817\end{array}$ & $\begin{array}{l}0.0787 \\
289.37 \\
1.1993\end{array}$ & $\begin{array}{l}0.0582 \\
298.38 \\
1.2132\end{array}$ \\
\hline
\end{tabular}




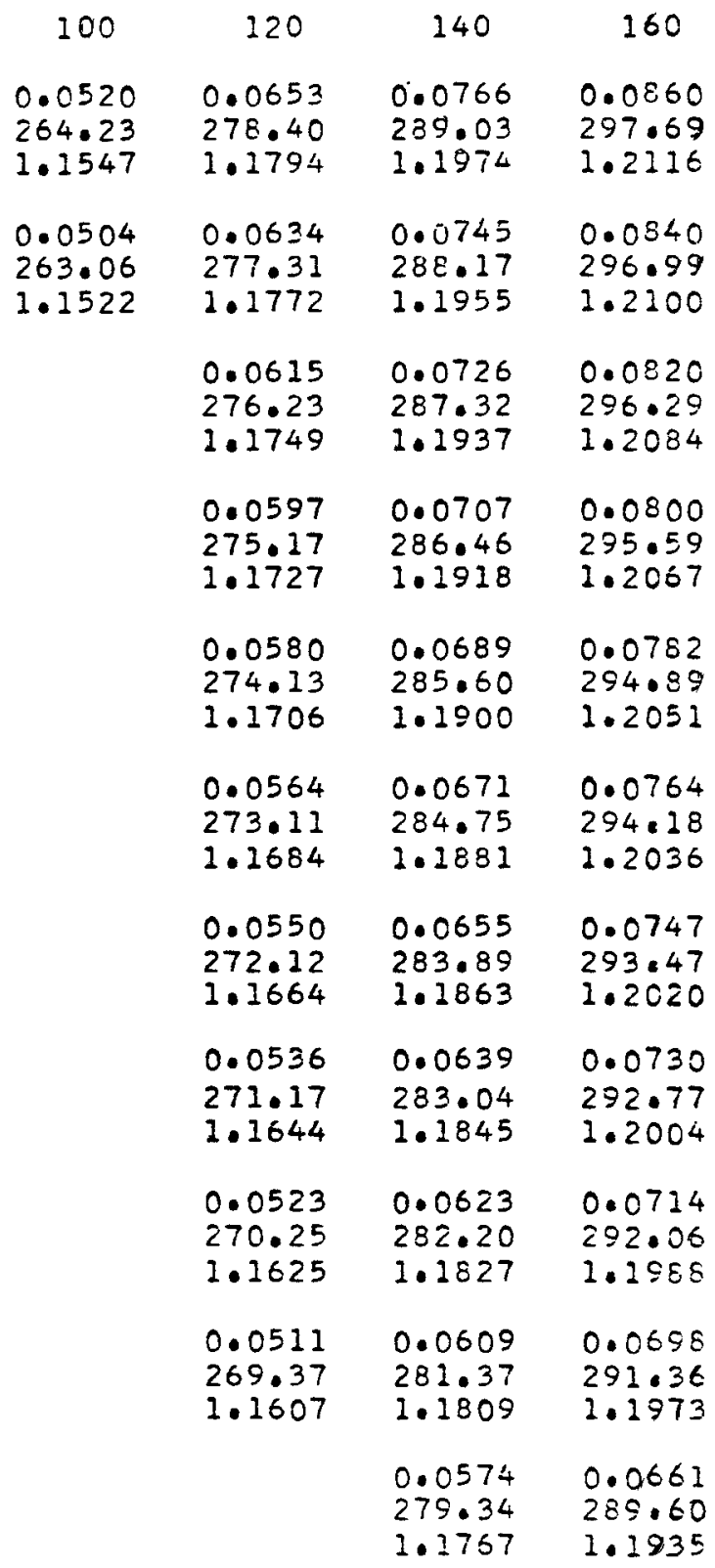




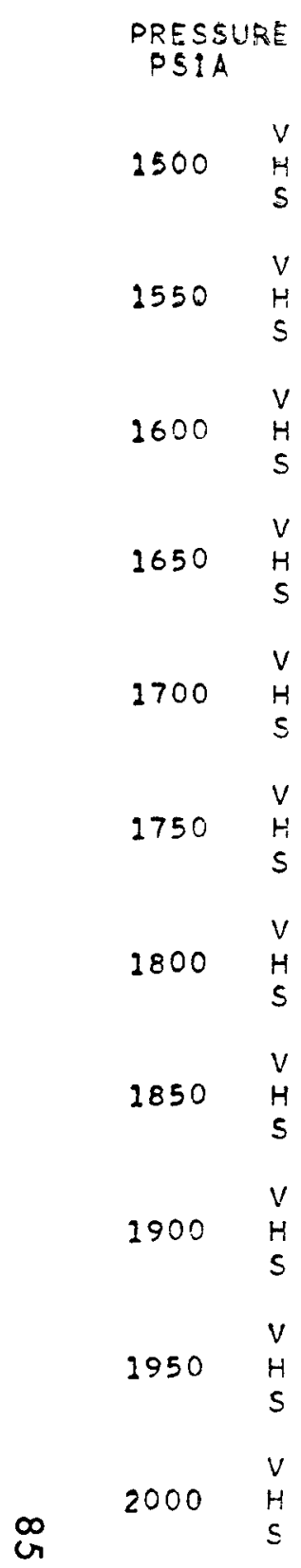




\begin{tabular}{|c|c|c|c|c|c|c|c|c|c|c|}
\hline 14.696 & $\begin{array}{l}\because \\
H \\
S\end{array}$ & $\begin{array}{r}10.5777 \\
334.69 \\
1.4566\end{array}$ & $\begin{array}{r}10,9117 \\
339<02 \\
1.4633\end{array}$ & $\begin{array}{r}11.2455 \\
343.40 \\
1.4698\end{array}$ & $\begin{array}{r}11.5792 \\
347082 \\
1.4762\end{array}$ & $\begin{array}{r}11.9127 \\
352.28 \\
1.4825\end{array}$ & $\begin{array}{r}12.2462 \\
356.79 \\
1.4887\end{array}$ & $\begin{array}{r}12.5795 \\
361.35 \\
1.4947\end{array}$ & $\begin{array}{r}12.9127 \\
365: 95 \\
105007\end{array}$ & $\begin{array}{r}13.2458 \\
370.59 \\
1.5066\end{array}$ \\
\hline 16 & $\begin{array}{l}V \\
H \\
S\end{array}$ & $\begin{array}{l}9.7132 \\
334.67 \\
1.4528\end{array}$ & $\begin{array}{r}10.0201 \\
339 \mathrm{c} 00 \\
1.4594\end{array}$ & $\begin{array}{r}10.3269 \\
343.37 \\
1.4660\end{array}$ & $\begin{array}{r}10.6336 \\
347.79 \\
1.4724\end{array}$ & $\begin{array}{r}10.9401 \\
352.26 \\
1.4787\end{array}$ & $\begin{array}{r}11.2465 \\
356.77 \\
1.4848\end{array}$ & $\begin{array}{r}11.5528 \\
361.33 \\
1.4909\end{array}$ & $\begin{array}{r}11: 8590 \\
365.93 \\
1.4969\end{array}$ & $\begin{array}{r}12.1651 \\
370.57 \\
1.5028\end{array}$ \\
\hline 20 & $\begin{array}{l}V \\
H_{i} \\
S\end{array}$ & $\begin{array}{l}7.7646 \\
334.59 \\
1.4427\end{array}$ & $\begin{array}{l}8.0107 \\
338.92 \\
1.4493\end{array}$ & $\begin{array}{l}8.2566 \\
343.30 \\
1.4559\end{array}$ & $\begin{array}{l}8.5023 \\
347.73 \\
1.4623\end{array}$ & $\begin{array}{l}8.7479 \\
352.20 \\
1.4686\end{array}$ & $\begin{array}{l}8.9934 \\
356.71 \\
1.4748\end{array}$ & $\begin{array}{l}9.2387 \\
361.27 \\
1.4808\end{array}$ & $\begin{array}{l}9.4840 \\
365.87 \\
1.4868\end{array}$ & $\begin{array}{l}9.7292 \\
370.52 \\
1.4927\end{array}$ \\
\hline 24 & $\begin{array}{l}V \\
H \\
S\end{array}$ & $\begin{array}{l}6.4656 \\
334.50 \\
1.4344\end{array}$ & $\begin{array}{l}6.6710 \\
338.84 \\
1.4411\end{array}$ & $\begin{array}{l}6.9763 \\
343.23 \\
1.4476\end{array}$ & $\begin{array}{l}7.0814 \\
347.66 \\
1.4540\end{array}$ & $\begin{array}{l}7.2864 \\
352.13 \\
1.4603\end{array}$ & $\begin{array}{l}7.4913 \\
356.65 \\
1.4665\end{array}$ & $\begin{array}{l}7.6960 \\
361.21 \\
1.4726\end{array}$ & $\begin{array}{l}7.9007 \\
365.82 \\
1.4786\end{array}$ & $\begin{array}{l}8.1052 \\
370.46 \\
1.4845\end{array}$ \\
\hline 28 & $\begin{array}{l}V \\
H \\
S\end{array}$ & $\begin{array}{l}5.5377 \\
334.42 \\
1.4274\end{array}$ & $\begin{array}{l}5.7142 \\
338.77 \\
1.4341\end{array}$ & $\begin{array}{l}5.8904 \\
343.16 \\
1.4406\end{array}$ & $\begin{array}{l}6.0665 \\
347.59 \\
1.4471\end{array}$ & $\begin{array}{l}6.2425 \\
352.07 \\
1.4534\end{array}$ & $\begin{array}{l}6.4183 \\
355059 \\
1.4595\end{array}$ & $\begin{array}{l}6.5941 \\
361.15 \\
1.4656\end{array}$ & $\begin{array}{l}6.7697 \\
365.76 \\
1.4716\end{array}$ & $\begin{array}{l}609453 \\
370041 \\
1.4775\end{array}$ \\
\hline 32 & $\begin{array}{l}V \\
H \\
S\end{array}$ & $\begin{array}{l}4.8418 \\
334.34 \\
1.4213\end{array}$ & $\begin{array}{l}409965 \\
338.69 \\
1.4280\end{array}$ & $\begin{array}{l}5.1510 \\
343.08 \\
1.4346\end{array}$ & $\begin{array}{l}5.3053 \\
34.7 .52 \\
1.4410\end{array}$ & $\begin{array}{l}5.4596 \\
352.00 \\
1.4473\end{array}$ & $\begin{array}{l}5.6136 \\
356.52 \\
1.4535\end{array}$ & $\begin{array}{l}5.7676 \\
361.09 \\
1.4596\end{array}$ & $\begin{array}{l}5.9215 \\
365070 \\
1.4656\end{array}$ & $\begin{array}{l}600753 \\
370036 \\
1.4714\end{array}$ \\
\hline 36 & $\begin{array}{l}V \\
H \\
S\end{array}$ & $\begin{array}{l}4.3006 \\
334.26 \\
1.4160\end{array}$ & $\begin{array}{l}404383 \\
338.62 \\
1.4226\end{array}$ & $\begin{array}{l}4.5750 \\
343.01 \\
1.4292\end{array}$ & $\begin{array}{l}4.7133 \\
347.45 \\
1.4356\end{array}$ & $\begin{array}{l}4.8506 \\
351.93 \\
1.4419\end{array}$ & $\begin{array}{l}4.9878 \\
356.46 \\
2.4481\end{array}$ & $\begin{array}{l}5.1248 \\
361.03 \\
1.4542\end{array}$ & $\begin{array}{l}5.2618 \\
365.65 \\
1.4602\end{array}$ & $\begin{array}{l}5.3987 \\
370.30 \\
1.4661\end{array}$ \\
\hline 40 & $\begin{array}{l}V \\
H \\
S\end{array}$ & $\begin{array}{l}3.8676 \\
334.18 \\
1.4111\end{array}$ & $\begin{array}{l}3.9918 \\
338.54 \\
1.4178\end{array}$ & $\begin{array}{l}4.1158 \\
342.94 \\
1.4244\end{array}$ & $\begin{array}{l}4.2397 \\
347.38 \\
1.4308\end{array}$ & $\begin{array}{l}4.3634 \\
351.87 \\
1.4371\end{array}$ & $\begin{array}{l}4.4871 \\
356.40 \\
1.4434\end{array}$ & $\begin{array}{l}4.6106 \\
360.97 \\
1.4494\end{array}$ & $\begin{array}{l}4.7340 \\
365.59 \\
1.4554\end{array}$ & $\begin{array}{l}4.8574 \\
370.25 \\
1.4613\end{array}$ \\
\hline 44 & $\begin{array}{l}V \\
H \\
S\end{array}$ & $\begin{array}{l}3.5133 \\
334.10 \\
1.4068\end{array}$ & $\begin{array}{l}3.6264 \\
338.46 \\
1.4135\end{array}$ & $\begin{array}{l}3.7394 \\
342.87 \\
1.4200\end{array}$ & $\begin{array}{l}3.8522 \\
347.31 \\
1.4265\end{array}$ & $\begin{array}{l}3.9649 \\
351.80 \\
1.4328\end{array}$ & $\begin{array}{l}4.0774 \\
356.34 \\
1.4390\end{array}$ & $\begin{array}{l}4.1899 \\
360092 \\
1.4451\end{array}$ & $\begin{array}{l}403022 \\
365.53 \\
164511\end{array}$ & $\begin{array}{l}4.4145 \\
370.20 \\
1.4570\end{array}$ \\
\hline 48 & $\begin{array}{l}V \\
H \\
S\end{array}$ & $\begin{array}{l}3.2181 \\
334.02 \\
1.4028\end{array}$ & $\begin{array}{l}303220 \\
338.39 \\
1.4095\end{array}$ & $\begin{array}{l}3.4257 \\
342079 \\
1.4161\end{array}$ & $\begin{array}{l}3.5293 \\
347=25 \\
1.4225\end{array}$ & $\begin{array}{l}3.6327 \\
351.74 \\
1.4288\end{array}$ & $\begin{array}{l}3.7360 \\
356.28 \\
1.4350\end{array}$ & $\begin{array}{l}3.8393 \\
360.86 \\
1.4411\end{array}$ & $\begin{array}{l}3.9424 \\
365.48 \\
1.4471\end{array}$ & $\begin{array}{l}4.0454 \\
370.14 \\
\text { J. } 4530\end{array}$ \\
\hline 52 & $\begin{array}{l}V \\
H \\
S\end{array}$ & $\begin{array}{l}2.9683 \\
333.94 \\
1.3991\end{array}$ & $\begin{array}{l}3.0644 \\
338.31 \\
1.4058\end{array}$ & $\begin{array}{l}3.1603 \\
342.72 \\
1=\div 124\end{array}$ & $\begin{array}{l}3.2560 \\
347.18 \\
1.4188\end{array}$ & $\begin{array}{l}3.3517 \\
351.67 \\
1.4252\end{array}$ & $\begin{array}{l}3.4472 \\
356.21 \\
1.4314\end{array}$ & $\begin{array}{l}3.5426 \\
360: 80 \\
1.4375\end{array}$ & $\begin{array}{l}3: 6379 \\
365: 42 \\
1=4435\end{array}$ & $\begin{array}{l}3.7331 \\
370,09 \\
1.4404\end{array}$ \\
\hline
\end{tabular}




\begin{tabular}{|c|c|c|c|c|c|c|c|c|c|c|}
\hline \multirow{2}{*}{\multicolumn{2}{|c|}{$\begin{array}{l}\text { PRESSURE } \\
\text { PSIA }\end{array}$}} & \multicolumn{9}{|c|}{ TEMPERATURE } \\
\hline & & 180 & 200 & 220 & 240 & 260 & 280 & 300 & 320 & 340 \\
\hline 56 & $\begin{array}{l}V \\
H \\
S\end{array}$ & $\begin{array}{l}2.7539 \\
333.86 \\
1.3957\end{array}$ & $\begin{array}{l}2.8436 \\
338.23 \\
1.4024\end{array}$ & $\begin{array}{l}2.9328 \\
342.65 \\
1.4090\end{array}$ & $\begin{array}{l}3.0218 \\
347.11 \\
1.4154\end{array}$ & $\begin{array}{l}3.1108 \\
351.61 \\
1.4218\end{array}$ & $\begin{array}{l}3.1996 \\
356.15 \\
1.4280\end{array}$ & $\begin{array}{l}3.2883 \\
360.74 \\
1.4341\end{array}$ & $\begin{array}{l}3.3769 \\
365.37 \\
1.4401\end{array}$ & $\begin{array}{l}3.4654 \\
370.03 \\
1.4460\end{array}$ \\
\hline 60 & $\begin{array}{l}V \\
H \\
S\end{array}$ & $\begin{array}{l}2.5683 \\
333.78 \\
1.3925\end{array}$ & $\begin{array}{l}2.6519 \\
338.15 \\
1.3992\end{array}$ & $\begin{array}{l}2.7356 \\
342.58 \\
1.4058\end{array}$ & $\begin{array}{l}2.8189 \\
347.04 \\
1.4123\end{array}$ & $\begin{array}{l}2.9020 \\
351.54 \\
1.4186\end{array}$ & $\begin{array}{l}2.9850 \\
356.09 \\
1.4248\end{array}$ & $\begin{array}{l}3.0679 \\
360.68 \\
1.4310\end{array}$ & $\begin{array}{l}3.1507 \\
365.31 \\
1.4370\end{array}$ & $\begin{array}{l}3.2334 \\
369.98 \\
1.4429\end{array}$ \\
\hline 70 & $\begin{array}{l}V \\
H \\
S\end{array}$ & $\begin{array}{l}2.1971 \\
333.57 \\
1.3853\end{array}$ & $\begin{array}{l}2.2691 \\
337.96 \\
1.3921\end{array}$ & $\begin{array}{l}2.3410 \\
342.39 \\
1.3987\end{array}$ & $\begin{array}{l}2.4129 \\
346.87 \\
1.4052\end{array}$ & $\begin{array}{l}2.4844 \\
351.38 \\
1.4115\end{array}$ & $\begin{array}{l}2.5558 \\
355.93 \\
1.4178\end{array}$ & $\begin{array}{l}2.6271 \\
360.53 \\
1.4239\end{array}$ & $\begin{array}{l}2.6983 \\
365.17 \\
1.4299\end{array}$ & $\begin{array}{l}2.7695 \\
369.85 \\
1.4358\end{array}$ \\
\hline 80 & $\begin{array}{l}V \\
H \\
S\end{array}$ & $\begin{array}{l}1.9187 \\
333.37 \\
1.3791\end{array}$ & $\begin{array}{l}1.9820 \\
337.77 \\
1.3859\end{array}$ & $\begin{array}{l}2.0452 \\
342.21 \\
1.3925\end{array}$ & $\begin{array}{l}2.1082 \\
346.69 \\
1.3990\end{array}$ & $\begin{array}{l}2.1713 \\
351.22 \\
1.4054\end{array}$ & $\begin{array}{l}2.2340 \\
355.78 \\
1.4116\end{array}$ & $\begin{array}{l}2.2966 \\
360.38 \\
1.4177\end{array}$ & $\begin{array}{l}2.3591 \\
365.03 \\
1.4238\end{array}$ & $\begin{array}{l}2.4215 \\
369.71 \\
1.4297\end{array}$ \\
\hline 90 & $\begin{array}{l}V \\
H \\
S\end{array}$ & $\begin{array}{l}1.7022 \\
333.16 \\
1.3736\end{array}$ & $\begin{array}{l}1.7587 \\
337.58 \\
1.3804\end{array}$ & $\begin{array}{l}1.8151 \\
342.03 \\
1.3870\end{array}$ & $\begin{array}{l}1.8714 \\
346.52 \\
1.3935\end{array}$ & $\begin{array}{l}1.9275 \\
351.05 \\
1.3999\end{array}$ & $\begin{array}{l}1.9836 \\
355.62 \\
1.4062\end{array}$ & $\begin{array}{l}2.0395 \\
360.23 \\
1.4123\end{array}$ & $\begin{array}{l}2.0952 \\
364.89 \\
1.4183\end{array}$ & $\begin{array}{l}2.1508 \\
369.58 \\
1.4243\end{array}$ \\
\hline 100 & $\begin{array}{l}V \\
H \\
S\end{array}$ & $\begin{array}{l}1.5289 \\
332.96 \\
1.3686\end{array}$ & $\begin{array}{l}1.5801 \\
337.38 \\
1.3754\end{array}$ & $\begin{array}{l}1.5311 \\
341.84 \\
1.3821\end{array}$ & $\begin{array}{l}1.6819 \\
346.34 \\
1.3886\end{array}$ & $\begin{array}{l}1.7326 \\
350.88 \\
1.3950\end{array}$ & $\begin{array}{l}1.7832 \\
355.46 \\
1.4013\end{array}$ & $\begin{array}{l}1.8338 \\
360.09 \\
1.4074\end{array}$ & $\begin{array}{l}1.8841 \\
364.74 \\
1.4135\end{array}$ & $\begin{array}{l}1.9343 \\
369.44 \\
1.4194\end{array}$ \\
\hline 110 & $\begin{array}{l}V \\
H \\
S\end{array}$ & $\begin{array}{l}1.3872 \\
332.75 \\
1.3641\end{array}$ & $\begin{array}{l}1.4339 \\
337.19 \\
1.3710\end{array}$ & $\begin{array}{l}1.4805 \\
341.66 \\
1.3776\end{array}$ & $\begin{array}{l}1.5269 \\
346.17 \\
1.3842\end{array}$ & $\begin{array}{l}1.5732 \\
350.72 \\
1.3906\end{array}$ & $\begin{array}{l}1.6193 \\
355.31 \\
1.3968\end{array}$ & $\begin{array}{l}1.6653 \\
359.93 \\
1.4030\end{array}$ & $\begin{array}{l}1.7114 \\
364.60 \\
1.4091\end{array}$ & $\begin{array}{l}1.7572 \\
369.31 \\
1.4150\end{array}$ \\
\hline 120 & $\begin{array}{l}V \\
H \\
S\end{array}$ & $\begin{array}{l}1.2691 \\
332.55 \\
1.3600\end{array}$ & $\begin{array}{l}1.3121 \\
336.99 \\
1.3668\end{array}$ & $\begin{array}{l}1.3550 \\
341.48 \\
1.3735\end{array}$ & $\begin{array}{l}1.3977 \\
346.00 \\
1.3801\end{array}$ & $\begin{array}{l}1.4403 \\
350.55 \\
1.3865\end{array}$ & $\begin{array}{l}1.4827 \\
355.15 \\
1.3928\end{array}$ & $\begin{array}{l}1.5251 \\
359.79 \\
1.3989\end{array}$ & $\begin{array}{l}1.5673 \\
364.46 \\
1.4050\end{array}$ & $\begin{array}{l}1.6095 \\
369.17 \\
1.4110\end{array}$ \\
\hline 130 & $\begin{array}{l}V \\
H \\
S\end{array}$ & $\begin{array}{l}1.1691 \\
332.34 \\
1.3562\end{array}$ & $\begin{array}{l}1.2090 \\
336.80 \\
1.3630\end{array}$ & $\begin{array}{l}1.2488 \\
341.29 \\
1.3697\end{array}$ & $\begin{array}{l}1.2884 \\
345.82 \\
1.3763\end{array}$ & $\begin{array}{l}1.3279 \\
350.39 \\
1.3827\end{array}$ & $\begin{array}{l}1.3672 \\
354.99 \\
1.3890\end{array}$ & $\begin{array}{l}1.4064 \\
359.64 \\
1.3952\end{array}$ & $\begin{array}{l}1.4455 \\
364.32 \\
1.4013\end{array}$ & $\begin{array}{l}1.4846 \\
369.04 \\
1.4073\end{array}$ \\
\hline 140 & $\begin{array}{l}V \\
H \\
S\end{array}$ & $\begin{array}{l}1.0834 \\
332.13 \\
1.3526\end{array}$ & $\begin{array}{l}1.1207 \\
336.60 \\
1.3505\end{array}$ & $\begin{array}{l}1.1578 \\
341.11 \\
1.3662\end{array}$ & $\begin{array}{l}1.1947 \\
345.65 \\
1.3728\end{array}$ & $\begin{array}{l}1.2315 \\
350.22 \\
1.3792\end{array}$ & $\begin{array}{l}1.2681 \\
354.84 \\
1.3855\end{array}$ & $\begin{array}{l}1.3047 \\
352.49 \\
1.3917\end{array}$ & $\begin{array}{l}1.3411 \\
364.17 \\
1.3978\end{array}$ & $\begin{array}{l}1.3774 \\
368.90 \\
1.4038\end{array}$ \\
\hline 150 & $\begin{array}{l}V \\
H \\
S\end{array}$ & $\begin{array}{l}1.0092 \\
331.92 \\
1.3493\end{array}$ & $\begin{array}{l}1.0441 \\
336.41 \\
1.3562\end{array}$ & $\begin{array}{l}1.0789 \\
340.92 \\
1.3629\end{array}$ & $\begin{array}{l}1.1135 \\
345.47 \\
1.3695\end{array}$ & $\begin{array}{l}1.1480 \\
350.06 \\
1.3759\end{array}$ & $\begin{array}{l}1.1823 \\
354.68 \\
1.3823\end{array}$ & $\begin{array}{l}1.2165 \\
359.34 \\
1.3885\end{array}$ & $\begin{array}{l}1.2506 \\
364.03 \\
1.3946\end{array}$ & $\begin{array}{l}1.2846 \\
368.76 \\
1.4006\end{array}$ \\
\hline
\end{tabular}




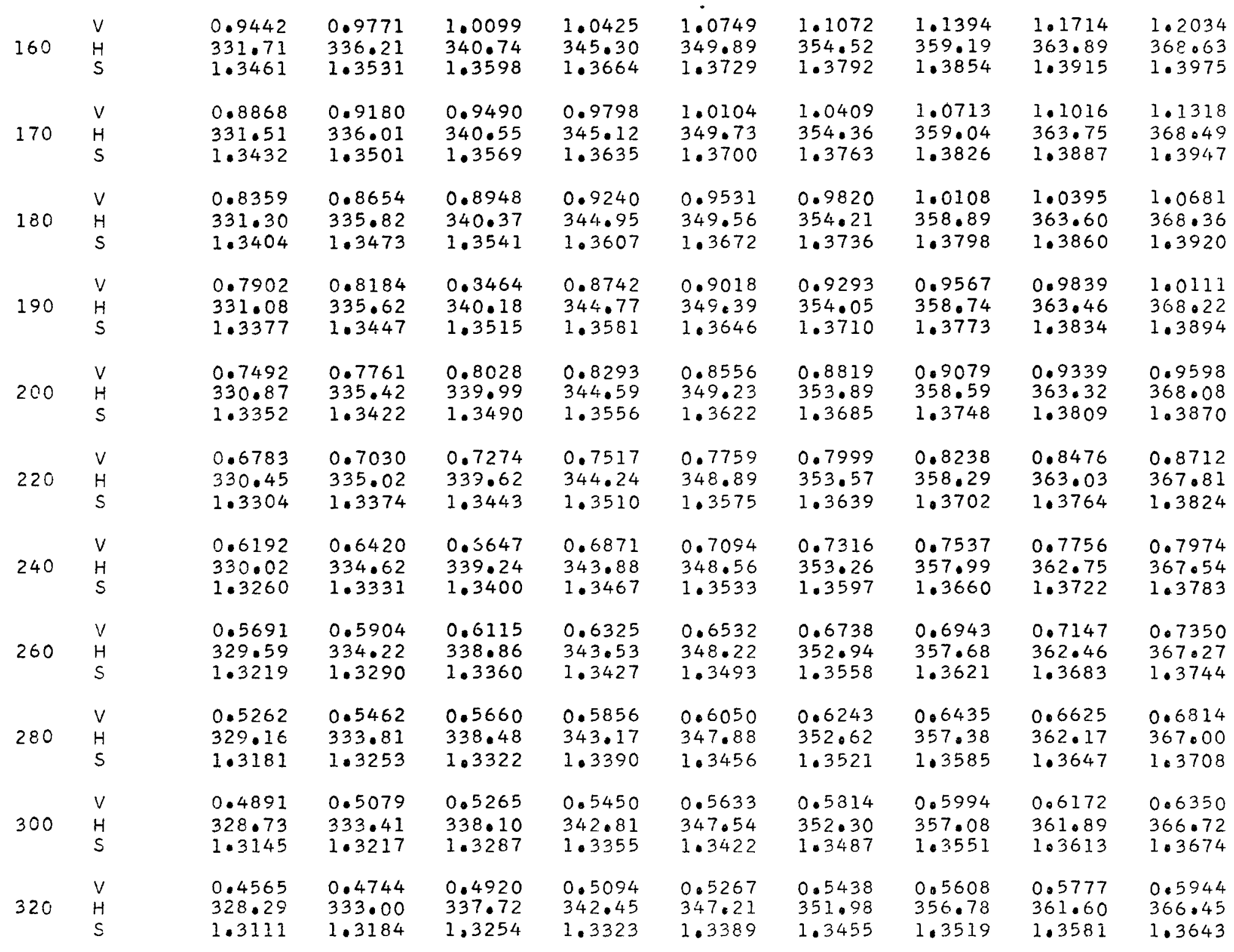


PRESSURE PSIA

180

$340 \quad \begin{array}{r}\mathrm{H} \\ \mathrm{S}\end{array}$

$360 \quad \mathrm{H}$

380

400

420

440

5

460

$\checkmark$

480

500

520

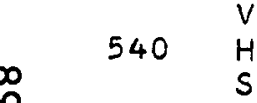

0.4278
327.85

0.4448 332.59

1.3152

1.3079

0.4023
327.40

1.3048

0.3794

326.96

1.3019

0.3588

326.51

I. 2991

0.3402

326.06

1. 2963

0.3232

325.60

1.2937

0.3078

325.15

1.2912

0.2936

324.69

1.2887

0.2805

$324 \cdot 22$

1.2864

0.2685

323.76

1.2841

0.2573

323.29

I. 2818

332.18

1. 3121

0.3949

331.76

1. 3093

0.3737

331.34

1.3065

0.3545

330.92

1.3038

0.3371

330.50

1.3013

0.3211

330.08

1.2988

0.3065

329.65

1.2964

0.2931

329.22

1. 2941

0.2807

328.79

I. 2918

0.2692

328.36

1.2896
200

220

0.4615

337.33

1. 3223

\subsection{5}

0.4344

336.95

1.3193

0.4102

335.56

1.3164

0.3883

336.17

1.3137

0.3686

335.78

1.3111

\subsection{6}

335.39

1.3085

0.3342

334.99

1.3061

0.3192

334.60

1.3038

0.3054

334.20

1.3015

0.2926

333.80

1.2993

0.2808

333.40

1.2971
TEMPERATURE

$240 \quad 260$

\subsection{1}

342.09

1.3291

0.4944
346.87

346.87

0.4502

341.73

0.4658

1.3262

346.52

I. 3329

0.4252

341.37

1.3234

0.4401

346.18

$0.4028 \quad 0.4170$

$341.00 \quad 345.84$

$1.3207 \quad 1.3275$

0.3825

340.63

0.3961

1.3181

1.3249

0.3640

340.27

0.3771

345.15

1.3156

1.3225

0.3471

339.90

0.3598

344.81

1. 3201

0.3316

339.53

0.3439

344.46

1. 3178

0.3174

339.16

0.3293

344.11

1.3087

1.3156

0.3043

338.79

1.3065

0.3158

343.76

1. 3135

0.2921

338.41

0.3033

1.3044

1. 3114
280

300

320

0.5427

361.31

1.3551

0.5117

361.02

1.3523

0.4839

360.74

1. 3495

0.4589

360.45

1.3469

0.4363

360.16

0.4157

359.87

1. 3421

0.3969

359.58

1. 3398
0.5107

351.66

1.3424

0.4812

351.34

1.3395

0.4548

351.01

I. 3368

0.4311

350.69

1. 3341

0.4096

350.37

1.3316

0.3901

350.04

1. 3292

0.3723

349.72

1. 3268

0.3560

349.39

1.3246

0.3409

349.06

1. 3224

0.3271

348.74

I. 3203

0.3142

348.41

I. 3183

0.5268

356.47

1. 3488

0.4965

356.17

1.3460

0.4694

355.86

1. 3432

0.4451

355.56

1.3406

0.4230

355.25

1. 3381

0.4030

354.95

1. 3357

0.3847

354.64

I. 3334

0.3679

354.33

1. 3312

0.3525

354.03

1.3290

0.3382

353.72

1. 3269

0.3250

353.41

1. 3249

366.18

1.3613

0.5268

365.90

1. 3584

0.4983

365.63

0.4726

365.36

0.4494

365.08

l. 3507

0.4283

$364 \cdot 81$

1.3483

0.4091

364.53

1.3461 
0.2698

\section{0}

260

280

300

320

340

1.2796

1.2951

0.2808

0.2916

0.3023

1.3024

343.06

348.08

1.3163

0.3128

353.10

0.3232

358.12

1.3230

1.3295

0.3334

$0.2373 \quad 0.2485$

580

1.2775

327.49

0.2595

0.2703

332.59

337.66

0.2808

1. 3004

342.71

0.2912

347.75

0.3014

352.79

0.3115

357.83

1.3276

0.2283

I. 2754

0.2393

0.2500

0.2605

337.28

0.2707

1.2834

33.18

1.2984

342.36

0.2808

347.42

0.2908

352.48

0.3006

357.54

1.3192

1.3258

$0.2198 \quad 0.2306$

0.2410

0.2513

0.2613

0.2711

0.2808

352.17

0.2903

357.25

1.3175

1.3240

0.2715

351.86

0.2808

356.95

I. 3223

1. 3089

1. 3157

0.2718

0.2627

$35 \mathrm{I} .55$

356.66

1.3140

1.3206

0.2544

351.23

0.2633

356.37

1. 3190

$\begin{array}{ll}312675 & 1.2757\end{array}$

330.55

335.76

340.94

346.10

1. 3123

$0.1908 \quad 0.200$

0.2104

0.2198

0.2289

0.2379

0.2467

350.92

0.2553

356.07

1.3107

1.3174

0.2393

0.2219

1.3038

0.2307

345.43

350.61

0.2478 355.78

1. 3158

0.2324

0.2239

345.09

350.30

0.2406

355.48

1.3076

1.3143

$0.1730 \quad 0.1825$

1.2784

1.2861

I. 2935

1.3007

0.2258

349.98

0.2339

355.19

344.76

1.3061

1.3128

$363 \cdot 16$

I. 3359

0.3214

362.88

1.3340

0.3102

362.60

1.3322

0.2998

362.33

1. 3305

0.2899

362.05

1. 3288

$0.280 ?$

361.77

1. 3271

0.2720

361.50

1. 3255

0.2638

361.22

1.3239

0.2561 360.94

1.3224

0.2488 360.67

1.3209

0.2419 360.39 1. 3194 


\begin{tabular}{|c|c|c|c|c|c|c|c|c|c|c|}
\hline \multicolumn{2}{|c|}{$\begin{array}{l}\text { PRESSURE } \\
\text { PSIA }\end{array}$} & 180 & \multicolumn{4}{|c|}{ TEMPERATURE } & 280 & 300 & 320 & 340 \\
\hline 780 & $\begin{array}{l}V \\
H \\
S\end{array}$ & $\begin{array}{l}0.1677 \\
317.40 \\
1.2584\end{array}$ & $\begin{array}{l}0.1770 \\
322.99 \\
1.2670\end{array}$ & $\begin{array}{l}0.1860 \\
328.45 \\
1.2751\end{array}$ & $\begin{array}{l}0.1947 \\
333.83 \\
1.2829\end{array}$ & $\begin{array}{l}0.2032 \\
339.15 \\
1.2904\end{array}$ & $\begin{array}{l}0.2114 \\
344.42 \\
1.2976\end{array}$ & $\begin{array}{l}0.2195 \\
349.67 \\
1.3046\end{array}$ & $\begin{array}{l}0.2275 \\
354.89 \\
1.3114\end{array}$ & $\begin{array}{l}0.2353 \\
360.11 \\
1.3179\end{array}$ \\
\hline 800 & $\begin{array}{l}V \\
H \\
S\end{array}$ & $\begin{array}{l}0.1626 \\
316.89 \\
1.2566\end{array}$ & $\begin{array}{l}0.1718 \\
322.53 \\
1.2653\end{array}$ & $\begin{array}{l}0.1807 \\
328.03 \\
1.2735\end{array}$ & $\begin{array}{l}0.1892 \\
333.44 \\
1.2813\end{array}$ & $\begin{array}{l}0.1975 \\
338.79 \\
1.2888\end{array}$ & $\begin{array}{l}0.2057 \\
344.09 \\
1.2961\end{array}$ & $\begin{array}{l}0.2136 \\
349.35 \\
1.3031\end{array}$ & $\begin{array}{l}0.2214 \\
354.60 \\
1.3099\end{array}$ & $\begin{array}{l}0.2291 \\
359.83 \\
1.3165\end{array}$ \\
\hline 820 & $\begin{array}{l}V \\
H \\
S\end{array}$ & $\begin{array}{l}0.1578 \\
316.37 \\
1.2549\end{array}$ & $\begin{array}{l}0.1669 \\
322.06 \\
1.2636\end{array}$ & $\begin{array}{l}0.1756 \\
327.61 \\
1.2719\end{array}$ & $\begin{array}{l}0.1840 \\
333.05 \\
1.2798\end{array}$ & $\begin{array}{l}0.1922 \\
338.42 \\
1.2873\end{array}$ & $\begin{array}{l}0.2002 \\
343.75 \\
1.2946\end{array}$ & $\begin{array}{l}0.2080 \\
349.04 \\
1.3017\end{array}$ & $\begin{array}{l}0.2156 \\
354.30 \\
1.3085\end{array}$ & $\begin{array}{l}0.2231 \\
359.55 \\
1.3151\end{array}$ \\
\hline 840 & $\begin{array}{l}V \\
H \\
S\end{array}$ & $\begin{array}{l}0.1532 \\
315.85 \\
1.2532\end{array}$ & $\begin{array}{l}0.1622 \\
321.59 \\
1.2620\end{array}$ & $\begin{array}{l}0.1708 \\
327.18 \\
1.2703\end{array}$ & $\begin{array}{l}0.1791 \\
332.66 \\
1.2783\end{array}$ & $\begin{array}{l}0.1871 \\
338.06 \\
1.2859\end{array}$ & $\begin{array}{l}0.1949 \\
343.41 \\
1.2932\end{array}$ & $\begin{array}{l}0.2026 \\
348.72 \\
1.3003\end{array}$ & $\begin{array}{l}0.2101 \\
354.01 \\
1.3071\end{array}$ & $\begin{array}{l}0.2175 \\
359.28 \\
1.3138\end{array}$ \\
\hline 860 & $\begin{array}{l}V \\
H \\
S\end{array}$ & $\begin{array}{l}0.1488 \\
315.33 \\
1.2515\end{array}$ & $\begin{array}{l}0.1577 \\
321.12 \\
1.2604\end{array}$ & $\begin{array}{l}0.1662 \\
326.75 \\
1.2688\end{array}$ & $\begin{array}{l}0.1743 \\
332.27 \\
1.2768\end{array}$ & $\begin{array}{l}0.1822 \\
337.70 \\
1.2844\end{array}$ & $\begin{array}{l}0.1899 \\
343.07 \\
1.2918\end{array}$ & $\begin{array}{l}0.1974 \\
348.41 \\
1.2989\end{array}$ & $\begin{array}{l}0.2048 \\
353.71 \\
1.3058\end{array}$ & $\begin{array}{l}0.2121 \\
359.00 \\
1.3125\end{array}$ \\
\hline 880 & $\begin{array}{l}V \\
H \\
S\end{array}$ & $\begin{array}{l}0.1447 \\
314.80 \\
1.2498\end{array}$ & $\begin{array}{l}0.1534 \\
320.65 \\
1.2588\end{array}$ & $\begin{array}{l}0.1618 \\
325.32 \\
1.2673\end{array}$ & $\begin{array}{l}0.1698 \\
331.87 \\
1.2753\end{array}$ & $\begin{array}{l}0.1776 \\
337.34 \\
1.2830\end{array}$ & $\begin{array}{l}0.1852 \\
342.73 \\
1.2904\end{array}$ & $\begin{array}{l}0.1926 \\
348.09 \\
1.2975\end{array}$ & $\begin{array}{l}0.1998 \\
353.42 \\
1.3044\end{array}$ & $\begin{array}{l}0.2069 \\
358.72 \\
1.3111\end{array}$ \\
\hline 900 & $\begin{array}{l}V \\
H \\
S\end{array}$ & $\begin{array}{l}0.1407 \\
314.27 \\
1.2482\end{array}$ & $\begin{array}{l}0.1493 \\
320.17 \\
1.2573\end{array}$ & $\begin{array}{l}0.1576 \\
325.89 \\
1.2658\end{array}$ & $\begin{array}{l}0.1655 \\
331.48 \\
1.2739\end{array}$ & $\begin{array}{l}0.1732 \\
336.97 \\
1.2816\end{array}$ & $\begin{array}{l}0.1806 \\
342.40 \\
1.2890\end{array}$ & $\begin{array}{l}0.1879 \\
347.78 \\
1.2962\end{array}$ & $\begin{array}{l}0.1950 \\
353.12 \\
1.3031\end{array}$ & $\begin{array}{l}0.2020 \\
358.44 \\
1.3099\end{array}$ \\
\hline 920 & $\begin{array}{l}V \\
H \\
S\end{array}$ & $\begin{array}{l}0.1368 \\
313.73 \\
1.2466\end{array}$ & $\begin{array}{l}0.1454 \\
319.69 \\
1.2557\end{array}$ & $\begin{array}{l}0.1535 \\
325.45 \\
1.2643\end{array}$ & $\begin{array}{l}0.1614 \\
331.08 \\
1.2725\end{array}$ & $\begin{array}{l}0.1689 \\
336.61 \\
1.2802\end{array}$ & $\begin{array}{l}0.1762 \\
342.06 \\
1.2877\end{array}$ & $\begin{array}{l}0.1834 \\
347.46 \\
1.2949\end{array}$ & $\begin{array}{l}0.1904 \\
352.82 \\
1.3018\end{array}$ & $\begin{array}{l}0.1973 \\
358.16 \\
1.3086\end{array}$ \\
\hline 940 & $\begin{array}{l}V \\
H \\
S\end{array}$ & $\begin{array}{l}0.1332 \\
313.20 \\
1.2450\end{array}$ & $\begin{array}{l}0.1416 \\
319.21 \\
1.2542\end{array}$ & $\begin{array}{l}0.1497 \\
325.02 \\
1.2629\end{array}$ & $\begin{array}{l}0.1574 \\
330.68 \\
1.2710\end{array}$ & $\begin{array}{l}0.1649 \\
336.24 \\
1.2789\end{array}$ & $\begin{array}{l}0.1721 \\
341.72 \\
1.2864\end{array}$ & $\begin{array}{l}0.1791 \\
347.14 \\
1.2936\end{array}$ & $\begin{array}{l}0.1860 \\
352.53 \\
1.3006\end{array}$ & $\begin{array}{l}0.1928 \\
357.88 \\
1.3073\end{array}$ \\
\hline 960 & $\begin{array}{l}V \\
H \\
S\end{array}$ & $\begin{array}{l}0.1296 \\
312.65 \\
1.2434\end{array}$ & $\begin{array}{l}0.1380 \\
318.73 \\
1.2527\end{array}$ & $\begin{array}{l}0.1460 \\
324.58 \\
1.2614\end{array}$ & $\begin{array}{l}0.1536 \\
330.28 \\
1.2697\end{array}$ & $\begin{array}{l}0.1610 \\
335.87 \\
1.2775\end{array}$ & $\begin{array}{l}0.1681 \\
341.37 \\
1.2851\end{array}$ & $\begin{array}{l}0.1750 \\
346.82 \\
1.2923\end{array}$ & $\begin{array}{l}0.1818 \\
352.23 \\
1.2993\end{array}$ & $\begin{array}{l}0.1885 \\
357.60 \\
1.3061\end{array}$ \\
\hline 980 & $\begin{array}{l}V \\
H \\
S\end{array}$ & $\begin{array}{l}0.1263 \\
312.11 \\
1.2418\end{array}$ & $\begin{array}{l}0.1346 \\
318.24 \\
1.2512\end{array}$ & $\begin{array}{l}0.1424 \\
324.14 \\
1.2600\end{array}$ & $\begin{array}{l}0.1500 \\
329.88 \\
1.2683\end{array}$ & $\begin{array}{l}0.1572 \\
335.50 \\
1.2762\end{array}$ & $\begin{array}{l}0.1643 \\
341.04 \\
1.2838\end{array}$ & $\begin{array}{l}0.1711 \\
346.51 \\
1.2911\end{array}$ & $\begin{array}{l}0.1778 \\
351.93 \\
1.2981\end{array}$ & $\begin{array}{l}0.1843 \\
357.33 \\
1.3049\end{array}$ \\
\hline
\end{tabular}




$\begin{array}{lll} & V & 0.1230 \\ 2000 \quad H & 311.56\end{array}$

0.1313

1.2402

317.75

0.1390

0.1465

323.70

329.48

0.1536

1.2586

1.2669

335.13

0.1199

311.00

0.1281

317.26

1.2483

0.1358

323.26

1.2572

0.1431

329.08

1.2656

1. 2749

0.1606

340.70

0.1673

1. 2825

346.19

1.2898

0.1739

351.64

1.2969

0.1804

357.05

1. 3037

\subsection{0 .1250}

$310.45 \quad 316.77$

0.1326

322.82

0.1399

328.68

0.1502

0.1570

334.76

340.35

0.1637

345.87

0.1702

351.34

I. 2957

0.1766

1.2736

1.2813

.2886

0.1666

334.39

0.1536

340.01

0.1602

345.55

1.2723

1.2800

1.2874

351.04

1.2945

356.77

1.3026

0.1140

0.1221

0.1296

0.1368

0.1437

0.1504

0.1568

1.2356

1.245

322.37

328.27

334.02

339.67

345.23

0.1632

350.74

1.2934

0.1729

356.49

1.3014

$\begin{array}{lll}0.1112 & 0.1192\end{array}$

0.1267

0.1338

1. 2862

0.1599

0.1536

333.65

0.147

344.91

350.44

$1.2850 \quad 1.2922$

0.1694

356.21

1.3003

$1.2340 \quad 1.2439$

1.2531

1.2617

1. 2698

1.2776

0.1505

0.1239

0.1309

0.1086

308.75
1.2325

0.1165

315.28

321.48

327.46

0.1377

333.28

0.1442

338.98

0.1567

1. 2764

1.2839

350.15

1.2911

0.1660

355.93

1.2992

0.2627

355.65

1. 2980

$\begin{array}{lll}0.1060 & 0.1138 & 0.1212\end{array}$

308.18

314.77

321.03

0.1282

0.1348

0.1413

327.05

332.91

338.64

0.1475

344.28

349.85

0.1595

355.37

1.2752

1. 2828

1.2900

1. 2970

$0.1035 \quad 0.1113 \quad 0.1186$

0.1255

0.1321

0.1385

0.1446

0.1506

343.96

349.55

1.2741

1.2816

1.2889

0.1565

355.09

1.2959

0.10110 .1088

0.1161

1.2579

0.1294

0.1357

0.1418

0.1477

337.95

349.25

1. 2878

0.1535

I. 2650

1.2729

1.2805

0.1450
348.95

0.1331

337.60

0.1391

343.32

1.2867

1.2948

$306.45 \quad 313.25$

319.67

325.82

331.78
1.2638

1.2718

1.2794

1.2867

0.1113

319.22

0.1180

325.41

0.1244

0.1306

331.41

337.26

0.1365

343.00

0.1423

348.65

1.2857

0.1507

354.53

1.2938

0.1480

354.26

1.2927 
PRESSURE PSIA

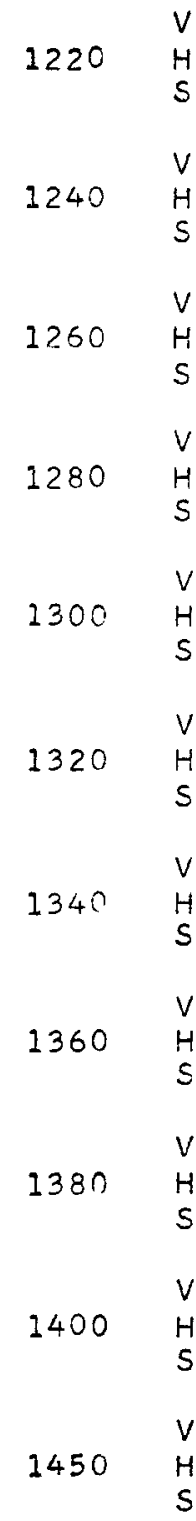

180

0.0944

305.28

1.2236

0.0923

304.69

1.2222

0.0902

304.10

1.2207

0.0883

303.51

1.2193

0.0864

302.91
1.2179

1.2179

0.0845

302.31

1.2164

0.0828

301.71

1.2150

0.0810

301.11
1.2136

0.0794

300.51

1.2122

0.0777

299.91

1.2108

0.0739

298.40

1.2074
0.1019

312.23

1. 2343

0.0998

311.72

1.2330

0.0977

311.20

1.2316

0.0957

310.68

1.2303

0.0938

310.17

1.2290

0.0919

309.65

1. 2277

0.0901

309.13

1.2264

0.0883

308.60

1.2251

0.0866

308.08

1.2238

0.0849

307.56

1.2226

0.0810

306.25

1. 2194
220

240

TEMPERATURE

0.1090

318.76

1.2440

0.1068

318.30

1.2428

0.1047

317.85

1. 2415

0.1026

317.39

1. 240 三

0.1005

316.93

I.2391

0.0987

315.46

1.2379

0.0968

316.00

1.2366

0.0950

315.54

1.2354

0.0932

315.08

1.2343

0.0915

314.61

1. 2331

0.0875

313.45

1. 2302

\subsection{7}

325.00

1.2530

0.1134

324.59

1.2519

0.1112

324.17

1.2507

0.1091

323.76

1.2495

0.1071

323.35

I. 2484

0.1051

322.93

1. 2472

0.1032

322.52

1. 2461

0.1013

322.10

1.2449

0.0995

321.68

1. 2438

0.0977

321.27

1. 2427

0.0935

320.22

1. 2400
260

0.1220

331.03

1. 2615

0.1197

330.66

1. 2604

0.1174

330.28

1.2593

0.1153

329.90

1. 2581

0.1132

329.52

I. 2570

0.1111

329.15

1.2559

0.1092

328.77

1. 2549

0.1072

328.39

1.2538

0.1054

328.01

1.2527

0.1036

327.63

1.2517

0.0993

326.69

1. 2491
280

300

320

340

0.1281

336.91

1.2696

0.1257

336.57

1.2685

0.1234

336.22

1.2674

0.1212

335.87

1. 2663

0.1190

335.53

1.2653

0.1169

335.18

1. 2542

0.1149

334.83

I. 2632

0.1129

334.49

1.2621

0.1110

334.14

1.2611

0.1092

333.79

1.2601

0.1048

332.92

1.2576
0.1340

342.68

1.2772

0.1316

342.36

1.2762

0.1292

342.04

1. 2751

0.1269

341.72

1.2741

0.1247

341.40

I. 2731

0.1225

341.08

1.2720

0.1205

340.75

1.2710

0.1184

340.43

1.2700

0.1165

340.11

1.2690

0.1146

339.79

I. 2681

0.1100

338.99

1.2657
0.1397

348.36

1.2846

0.1372

348.06

1.2836

0.1348

347.76

1.2826

0.1324

347.47

1.2816

0.1302

347.17

1.2806

0.1280

346.87

1.2796

0.1258

346.57

1.2786

0.1238

346.27

1.2776

0.1217

345.97

1.2767

351.75

0.1198

345.68

1.2757

0.1248

0.1151

344.93

1.2734 
$V$

0.0704

0.0773

304.93

0.0837

240

260

280

300

320

340

2500

1. 2040

1.2164

312.29

0.0897

0.0953

319.18

325.74

0.1007

0.1058

332.05

338.19

0.1108

344.19

0.1156

1.2273

.2373

1.2465

1.2551

1.2633

1.2711

350.08

$\begin{array}{lll}0.0671 & 0.0739 & 0.0802\end{array}$

1550

1.2007

303.63

311.13

0.0860

318.14

0.0916

324.79

0.0968

331.19

0.1019

337.39

0.1067

343.45

1.2785

1600

0.06410 .0708

0.0769

$293.95 \quad 302.33$

309.97

0.0826

1.2440

1.2527

1.2610

1.2689

0.1115

349.39

1.2764

$1.1975 \quad 1.2104$

1.2217

317.10

$\begin{array}{ll}0.0881 & 0.0932\end{array}$

0.0982

0.1029

330.32

336.59

342.71

0.1076

1.24151 .2504

1.2587

1.2667

348.70

0.0738

0.0795

0.0848

0.0899

$292.51 \quad 301.04$

308.82

316.06

322.90

329.46

0.0947

0.0994

1.2742

1.1944

1.2075

1.2295

1.2391

1.2481

335.80

341.97

0.1039

0.0710

0.0765

0.0817

0.0867

307.68

315.03

1.2368

328.60

1.2565

1.2645

348.01
1.2722

0.0915

0.0960

335.00

0.0960
341.23

$1.1913 \quad 1.2046$

0.0683

0.0737

$0.0564 \quad 0.0626$

$289.75 \quad 298.52$

305.55

314.00

0.0789

321.03

1.2458

1.2544

1.2624

0.1005

1.2246

1.2345

0.0837

327.74

0.0884

334.21

0.0929

340.50

$0.0543 \quad 0.0602$

0.0658

0.0711

0.0762

0.0809

1.2522

1.2604

0.0972

$288.44 \quad 297.30 \quad 305.43$

312.99

320.10

326.89

0.0855

333.42

0.0899

339.77

346.64

1.2113

1.2222

1.2322

1.2415

1.2502

1.2584

0.0942

$0.0687 \quad 0.0736$

0.0783

0.0828

0.0871

34596

304.33

311.98

319.18

326.04

332.64

339.04

0.0913

$1.1828 \quad 1.1965 \quad 1.2088$

1.2198

1.2299

1.2393

1.2481

1.2564

$345 \cdot 28$

0.0613

0.0664

0.0712

0.0758

0.0802

0.0845

331.86

338.31

1.2643

$\begin{array}{ll}286.00 & 294.95 \\ 1.1803 & 1.1940\end{array}$

1.2063

1.2175

1.2278

1.2372

1.2461

I. 2545

0.0886

0.0642

0.0690

0.0735

0.0778

331.09

0.0820

337.59

344.60

1.2624

$\begin{array}{llll}302.20 & 310.01 & 317.36 & 324.36 \\ 1.2040 & 1.2153 & 1.2256 & 1.2352\end{array}$

1. 2441

1.2526

0.0860

0.0755

0.0668

0.0713

309.04

316.47

323.54

330.32

0.0796

343.93

301.16

1.2131

1.2235

1. 2332

1.2422

336.88

0.0835

343.26

1. 2588 


\begin{tabular}{|c|c|c|c|c|c|c|c|c|c|c|}
\hline \multirow{2}{*}{\multicolumn{2}{|c|}{$\begin{array}{l}\text { PRESSURE } \\
\text { PSIA }\end{array}$}} & \multicolumn{9}{|c|}{ TEMPERATURE } \\
\hline & & 360 & 380 & 400 & 420 & 440 & 460 & 480 & 500 & 520 \\
\hline 4.696 & $\begin{array}{l}V \\
H \\
S\end{array}$ & $\begin{array}{r}13.5789 \\
375.27 \\
1.5124\end{array}$ & $\begin{array}{r}13.9118 \\
380.00 \\
1.5181\end{array}$ & $\begin{array}{r}14.2447 \\
384.76 \\
1.5237\end{array}$ & $\begin{array}{r}14.5776 \\
389.57 \\
1.5292\end{array}$ & $\begin{array}{r}14.9104 \\
394.41 \\
1.5346\end{array}$ & $\begin{array}{r}15.2431 \\
399.30 \\
1.5400\end{array}$ & $\begin{array}{r}15.5758 \\
404.21 \\
1.5453\end{array}$ & $\begin{array}{r}15.9084 \\
409.17 \\
1.5505\end{array}$ & $\begin{array}{r}16.2410 \\
414.16 \\
1.5556\end{array}$ \\
\hline 16 & $\begin{array}{l}V \\
H \\
S\end{array}$ & $\begin{array}{r}12.4711 \\
375.26 \\
1.5085\end{array}$ & $\begin{array}{r}12.7771 \\
379.98 \\
1.5142\end{array}$ & $\begin{array}{r}13.0829 \\
384.75 \\
1.5198\end{array}$ & $\begin{array}{r}13.3888 \\
389.56 \\
1.5254\end{array}$ & $\begin{array}{r}13.6945 \\
394.40 \\
1.5308\end{array}$ & $\begin{array}{r}14.0002 \\
399.28 \\
1.5362\end{array}$ & $\begin{array}{r}14.3058 \\
404.20 \\
1.5414\end{array}$ & $\begin{array}{r}14.6114 \\
409.16 \\
1.5467\end{array}$ & $\begin{array}{r}14.9170 \\
414.15 \\
1.5518\end{array}$ \\
\hline 20 & $\begin{array}{l}V \\
4 \\
S\end{array}$ & $\begin{array}{l}9.9743 \\
375.21 \\
1.4985\end{array}$ & $\begin{array}{r}10.2193 \\
379.93 \\
1.5042\end{array}$ & $\begin{array}{r}10.4643 \\
384.70 \\
1.5098\end{array}$ & $\begin{array}{r}10.7091 \\
389.51 \\
1.5153\end{array}$ & $\begin{array}{r}10.9540 \\
394.36 \\
1.5207\end{array}$ & $\begin{array}{r}11.1987 \\
399.24 \\
1.5261\end{array}$ & $\begin{array}{r}11.4435 \\
404.16 \\
1.5314\end{array}$ & $\begin{array}{r}11.6881 \\
409.12 \\
1.5366\end{array}$ & $\begin{array}{r}11.9328 \\
414.11 \\
1.5418\end{array}$ \\
\hline 24 & $\begin{array}{l}V \\
H \\
S\end{array}$ & $\begin{array}{l}8.3097 \\
375.15 \\
2.4902\end{array}$ & $\begin{array}{l}8.5141 \\
379.88 \\
1.4959\end{array}$ & $\begin{array}{l}8.7185 \\
384.66 \\
1.5016\end{array}$ & $\begin{array}{l}8.9227 \\
389.47 \\
1.5071\end{array}$ & $\begin{array}{l}9.1269 \\
394.31 \\
1.5125\end{array}$ & $\begin{array}{l}9.3311 \\
399.20 \\
1.5179\end{array}$ & $\begin{array}{l}9.5352 \\
404.12 \\
1.5232\end{array}$ & $\begin{array}{l}9.7393 \\
409.08 \\
1.5284\end{array}$ & $\begin{array}{l}9.9433 \\
414.07 \\
1.5335\end{array}$ \\
\hline 28 & $\begin{array}{l}V \\
H \\
S\end{array}$ & $\begin{array}{l}7.1208 \\
375.10 \\
1.4833\end{array}$ & $\begin{array}{l}7.2961 \\
379.84 \\
1.4890\end{array}$ & $\begin{array}{l}7.4715 \\
384.61 \\
1.4946\end{array}$ & $\begin{array}{l}7.6467 \\
389.42 \\
1.5001\end{array}$ & $\begin{array}{l}7.8219 \\
394.27 \\
1.5056\end{array}$ & $\begin{array}{l}7.9971 \\
399.16 \\
1.5109\end{array}$ & $\begin{array}{l}8.1722 \\
404.08 \\
1.5162\end{array}$ & $\begin{array}{l}8.3472 \\
409.04 \\
1.5214\end{array}$ & $\begin{array}{l}8.5222 \\
414.04 \\
1.5266\end{array}$ \\
\hline 32 & $\begin{array}{l}V \\
H \\
S\end{array}$ & $\begin{array}{l}6.2290 \\
375.05 \\
1.4772\end{array}$ & $\begin{array}{l}6.3827 \\
379.79 \\
1.4829\end{array}$ & $\begin{array}{l}6.5362 \\
384.56 \\
1.4886\end{array}$ & $\begin{array}{l}6.6897 \\
389.38 \\
1.4941\end{array}$ & $\begin{array}{l}6.8432 \\
394.23 \\
1.4995\end{array}$ & $\begin{array}{l}6.9966 \\
399.12 \\
1.5049\end{array}$ & $\begin{array}{l}7.1499 \\
404.04 \\
1.5102\end{array}$ & $\begin{array}{l}7.3032 \\
409.00 \\
1.5154\end{array}$ & $\begin{array}{l}7.4564 \\
414.00 \\
1.5206\end{array}$ \\
\hline 36 & $\begin{array}{l}V \\
H \\
S\end{array}$ & $\begin{array}{l}5.5355 \\
375.00 \\
1.4719\end{array}$ & $\begin{array}{l}5.6722 \\
379.74 \\
1.4775\end{array}$ & $\begin{array}{l}5.8088 \\
384.51 \\
1.4832\end{array}$ & $\begin{array}{l}5.9454 \\
389.33 \\
1.4888\end{array}$ & $\begin{array}{l}6.0819 \\
394.18 \\
1.4942\end{array}$ & $\begin{array}{l}6.2184 \\
399.08 \\
1.4996\end{array}$ & $\begin{array}{l}6.3548 \\
404.00 \\
1.5049\end{array}$ & $\begin{array}{l}6.4912 \\
408.97 \\
1.5101\end{array}$ & $\begin{array}{l}6.6275 \\
413.97 \\
1.5153\end{array}$ \\
\hline 40 & $\begin{array}{l}V \\
H \\
S\end{array}$ & $\begin{array}{l}4.9806 \\
374.95 \\
1.4671\end{array}$ & $\begin{array}{l}5.1038 \\
379.69 \\
1.4728\end{array}$ & $\begin{array}{l}5.2269 \\
384.47 \\
1.4785\end{array}$ & $\begin{array}{l}5.3499 \\
389.29 \\
1.4840\end{array}$ & $\begin{array}{l}5.4729 \\
394.14 \\
1.4894\end{array}$ & $\begin{array}{l}5.5958 \\
399.03 \\
1.4948\end{array}$ & $\begin{array}{l}5.7187 \\
403.96 \\
1.5001\end{array}$ & $\begin{array}{l}5.8415 \\
408.93 \\
1.5053\end{array}$ & $\begin{array}{l}5.9643 \\
413.93 \\
1.5105\end{array}$ \\
\hline 44 & $\begin{array}{l}V \\
H \\
S\end{array}$ & $\begin{array}{l}4.5266 \\
374.90 \\
1.4628\end{array}$ & $\begin{array}{l}4.6387 \\
379.64 \\
1.4685\end{array}$ & $\begin{array}{l}4.7508 \\
384.42 \\
1.4741\end{array}$ & $\begin{array}{l}4.8627 \\
389.24 \\
1.4797\end{array}$ & $\begin{array}{l}4.9746 \\
394.10 \\
1.4851\end{array}$ & $\begin{array}{l}5.0865 \\
398.99 \\
1.4905\end{array}$ & $\begin{array}{l}5.1983 \\
403.92 \\
1.4958\end{array}$ & $\begin{array}{l}5.3100 \\
408.89 \\
1.5010\end{array}$ & $\begin{array}{l}5.4217 \\
413.89 \\
1.5062\end{array}$ \\
\hline 48 & $\begin{array}{l}V \\
H \\
S\end{array}$ & $\begin{array}{l}4.1483 \\
374.85 \\
1.4588\end{array}$ & $\begin{array}{l}4.2512 \\
379.59 \\
1.4646\end{array}$ & $\begin{array}{l}4.3540 \\
384.37 \\
1.4702\end{array}$ & $\begin{array}{l}4.4567 \\
389.20 \\
1.4757\end{array}$ & $\begin{array}{l}4.5594 \\
394.06 \\
1.4812\end{array}$ & $\begin{array}{l}4.6620 \\
398.95 \\
1.4866\end{array}$ & $\begin{array}{l}4.7646 \\
403.88 \\
1.4919\end{array}$ & $\begin{array}{l}4.8671 \\
408.85 \\
1.4971\end{array}$ & $\begin{array}{l}4.9696 \\
413.86 \\
1.5022\end{array}$ \\
\hline 52 & $\begin{array}{l}V \\
H \\
S\end{array}$ & $\begin{array}{l}3.8282 \\
374.79 \\
1.4552\end{array}$ & $\begin{array}{l}3.9233 \\
379.54 \\
1.4609\end{array}$ & $\begin{array}{l}4.0183 \\
384.33 \\
1.4666\end{array}$ & $\begin{array}{l}4.1132 \\
389.15 \\
1.4721\end{array}$ & $\begin{array}{l}4.2081 \\
394.01 \\
1.4776\end{array}$ & $\begin{array}{l}4.3029 \\
398.91 \\
1.4829\end{array}$ & $\begin{array}{l}4.3976 \\
403.85 \\
1.4882\end{array}$ & $\begin{array}{l}4.4923 \\
408.82 \\
1.4935\end{array}$ & $\begin{array}{l}4.5870 \\
413.82 \\
1.4986\end{array}$ \\
\hline
\end{tabular}


$56 \quad \mathrm{H}$

3.5539

3.6422

$374.74 \quad 379049$

1.4518

379.49
1.4576

3.7305

420

440

460

480

500

520

384.28

$3.8187 \quad 3.9069$

$389.11 \quad 393.97$

1.4687

1.4742

3.9950

4.0831

403.81

4.1711

408.78

1.4796

1.4849

1.4901

4.2591

$413 \cdot 78$

3.4811

$\begin{array}{ll}3.3161 & 3.3986 \\ 374.69 & 379.44\end{array}$

$1.4487 \quad 1.4544$

384.23

3.5635

3.6459

389.06

393.93

3.7282

398.83

3.8105

403.77

3.8927

408.74

1.4870

$2.8405 \quad 2.9114$

$\begin{array}{ll}374.56 & 379.32 \\ 1.4416 & 1.4474\end{array}$

2.9823

3.0531

1.4711

1.4764

1.4817

3.3359

3.2653

388.95

3.1239
393.82

3.1946

384.11

1.4586

1.4640

1.4694

1.4747

408.64

1.4800

3.9748

413.75

1.4921

$2.4838 \quad 2.5460$

2.6082

2.6703

2.7324

2.7944

3.4064

413.66

1.4851

1.43551 .4413

384.00

388.84

393.71

398.62

2.8563

403.57

2.9182

408.55

2.9801

1.4633

1.4686

1.4739

$2.2064 \quad 2.2619$

2.3173

2.3726

2.4279

2.4831

2.5383

403.47

2.5934

413.56

1.4791

$383.88 \quad 388.72 \quad 393.60 \quad 398.52$

$1.4471 \quad 1.4526 \quad 1.4580$

1.4633

408.45

2.6485

$1.4301 \quad 1.4359$

.4415

2.1344

2.1643

2.2341

2.2839

1.4685

413047

1.4737

$1.9844 \quad 2.0345$

2.0845

388.61

393.50

398.42

403.37

2.3336

408.36

2.3833

1.4367

1.4422

1.4477

1.4585

1.4637

413.38

1.4689

$1.8029 \quad 1.8485$

$374.05 \quad 378.83$

1.3940

1.9395

1.9850

383.64

388.50

393.39

2.0304

398.31

2.0757

403.27

2.1210

408.27

$2 \cdot 1662$

1.4488

1.4541

1.4593

413.29

1.9022

403.17

1.9438

408.17

1.9854

398.21

1.4501

1.4553

413.20

1.4283

1.4338

1.4393

1.4448

1.7554

1.7939

1.4605

$1.5235 \quad 1.5623$

1.6011

1.6397

1.6784

1.7169

403.07

408.08

1.8323

1.4246

1.4301

1.4356

1.4411

1.4464

1.4517

413.11

1.4569

$1.4138 \quad 1.4499$

$\begin{array}{ll}373.66 & 378.46\end{array}$

1.4860

1.5220

1.5579

1.5938

383.29
1.4211

388.16

393.06

1.6296

1.6654

402.98
1.4430

407.98
1.4483

1.7012

1.4376

413.02
1.4534

1.5206

402.88

1.5540

407.89

1.5875

392.96

1.4871

397.90

1.4398

1.4451

1.4503 
PRESSURE PSIA

160

170

180

190

200

$\checkmark$

220

360

380

400

420

440

$1.2353 \quad 1.2672$

1.4034

378.21
1.4092

1.1619

373.27

1.1920

378.09

1.4064

1.0966

373.14

1.1250

377.96

1.4037

1.038

373.01

1.0652

377.84

1. 4011

0.9856

372.88

1.0113

377.72

1. 3987

0.8948

0.9183

377.47

1. 3942

1.3884

0.8192

372.37

0.8408

377.22

1.3901

0.755

372.11

0.7752

376.98

1.3862

0.7003

371.85

0.7190

376.73

1.3827

0.6703

376.48

371.59

1.3734

1.3793

0.6111

371.33

0.6277

376.23

1.3762
1.2989

383.06

1.4149

1. 2219

382.94

1.4121

1.1534

382.82

1.4094

1.0922

382.71

1.4069

1.0371

382.59

I. 4044

0.9417

382.35

1. 3999

0.3624

382.11

1.3958

0.7952

381.88

1.3920

0.7377

381.64

1.3884

0.6878

381.41

1. 3851

0.6442

381.17

1.3820

\subsection{6}

387.94

1.4205

1.2518

387.82

1.4177

1. 1817

387.71

1.4150

1.1190

387.60

1.4125

1.0626

387.49

1.4101

0.9652

387.26

1.4056

0.8839

387.03

1.4015

0.8152

386.81

1.3977

0.7563

386.58

1. 3941

0.7053

386.36

1.3908

391.34

0.6606

386.13

1.3877

0.6770

391.13

1.3933

1.3622

1.4260

1.2816

392.74
1.4232

1. 2099

1.4205

1.1458

2.53

1.0881

392.42

1.4156

0.9885

392.21

0.9054

391.99

0.8352

391.78

1.4032

0.7749

391.56

1.3997
460

480

500

520

1

0.7934

396.56

1.4052

0.7400

396.36

1.4019

0.6933

396.15

1.3988
1.4252

402.78

$\therefore 4368$

1.4566 407.79

1. 4421

1.4880

412.84

1.4473

1.3410

402.68

1.3706

407.70

1.4002

412.75

1.4445

1.2661

2942

402.58

407.60

1.3222

$412 \cdot 66$

1.4313

1.4366

1.4418

1. 1992

1.2258

1.2524

402.48

407.51

412.57

1. 4288

1.4341

1.4393

1.1389

1. 1643

$1 \cdot 1896$

402.38

407.41

$412 \cdot 48$

1.4264

1.4317

1.4369

1.0349

402.19

1.0580

407.23

1.0811

1.4220

1.4273

0.9481

401.99

0.9694

407.04

0.9906

1.4179

1.4232

412.11

0.8747

0.8944

406.85

0.9141

1.4141

1.4194

411.93

0.8118

0.8302

401.60

406.66

0.8485

1.4106

1.4159

411.75

0.7573

0.7745

401.40

406.47

0.7917

1.407

1.4127

0.7096

0.7258

401.21

406.29

0.7419

1.4042

1.4096

1.4149 
0.6155

0.6301 1.4067

1

1.3645

1.3704

1.3762

1.3820

1.3876

$395 \cdot 74$

400.81

0.6446

405.91

0.6590

1.3931

1.3986

1.4040

411.03

$\begin{array}{lll}0.5125 & 0.5267 & 0.5408\end{array}$

$\begin{array}{lll}370.55 & 375.49 & 380.46\end{array}$

0.5548

0.5688

385.46

390.48

0.5827

395.54

0.5966

1.3793

1.3850

1.3905

400.62

0.6104

405.72

1.4014

\subsection{3}

370.29

0.4998

375.24

0.5132

380.23

0.5266

385.23

0.5400

390.27

0.5532

395.33

0.5664

400.42

0.5796

405.54

1.3989

0.6241

410.86

1.4066

$0.4625 \quad 0.4754$

$370.03 \quad 375.00$

0.4883

379.99

0.5011

0.5139

385.01

390.05

0.5265

395.13

0.5392

1.3568

1.3628

1.3686

1.3744

1.3801

1.3356

0.4409

0.4533

0.4656

0.4779

0.4901

0.5023

394.92

38.34
1.3778

1.3834

400.22

1.3911

0.5518

405.35

1.3965

0.5927

410.68

1.4042

0.5144

400.03

0.5265

405.16

1.3721

0.4685

$0.4211 \quad 0.4331$

369.51

0.4331
374.50

$0 .+449$

0.4567

379.52
1.3641

384.56
1.3699

389.63

0.4801

394.72

1.3889

1.3943

0.5643

410.50

1.4018

0.4030

0.4145

0.4250

$374.25 \quad 379.28$

0.4373

384.34

0.4486

389.41

0.4918

399.83

0.5034

404.98

1.3921

0.5385

410.32

1.3996

0.4598

394.51

0.4710

399.64

0.4821

404.78

1.3900

0.5149

410.14

1.3974

0.4933 409.96 1.3953

0.3864

0.3975

0.4085

379.05

0.4195

0.4303

384.11

389.20

0.4412

394.31

0.4519

399.44

0.4627

404.60

0.4734

$1.3479 \quad 1.3540 \quad 1.3599$

1.3657

1.3714

1.3771

1.3826

1.3880

$409 \cdot 78$

$\begin{array}{lll}0.3710 & 0.3817 & 0.3924\end{array}$

0.4030

0.4135

0.4239

0.4343

399.24

0.4447

404.41

388.98
1.3695

394.10

1.3806

1.3861

0.4550

409.60

1.3638

0.3979

0.4080

0.4180

0.3877

388.77

393.90

399.05

0.4280

404.22

1.3914

368.46
1.3440

373.51
1.3501

1.3560

1.3618

1.3676

1.3787 
PRESSURE PSIA

$\begin{array}{ll} & \\ 5 & \\ & H \\ & S\end{array}$

580

600

620

640

660

680

700

720

$740 \quad H$

$\begin{array}{lll} & & V \\ 0 & 760 & H \\ & & S\end{array}$

\section{6}

0.3436

\section{$368 \cdot 20$}

1.3421

0.3313

367.94

0.3198

367.68

0.3091

1.3367

0.3080

0.2990

367.16

0.2896

366.89

1.3334

0.2807

366.63

$0.2723 \quad 0.2806$

0.2643

366.11

0.2568

355.85

1. 3273

0.2497
365.58

1. 3258
0.3536

373.26

1. 3482

0.3411

373.01

1. 3464

0.3293

0.3293
372.77

1.3446

0.3183
372.52

1.3429

372.27

I. 3412

0.2983

372.02

1.3396

0.2892

371.77

1. 3380

371.53
1.3365

0.2725

371.28

I. 3350

371.03

1. 3335

400

0.3636

378.34

1.3541

0.3507

378.10

1.3523

0.3387

377.87

1.3506

0.3274

377.63

1.3489

\subsection{9}

377.40

0.3070

377.16

1.3456

0.2976

376.93

1. 3441

0.2888

376.69

1. 3425

0.2805

376.46

0.2727

376.22

I. 3396

0.2575
370.78

0.2652

1. 3321

1.3382
1. 3472

1. 3411

TEMPERATURE
$420 \quad 440$

$0.3735 \quad 0.3834$

$383.44 \quad 388.56$

1.3600

1.3657

0.3603

383.21

1. 3582

0.3699

$388 \cdot 34$

1.3640

0.3480

382.99

1.3565

0.3573

388.13

1.3622

0.3365

382.77

0.3455

387.91

1.3606

0.3257

382.54

0.3344

387.70

I. 3532

1.3589

0.3155

382.32

1. 3516

0.3241

387.49

1.3574

0.3060

382.09

0.3143

387.27

1.3500

1.3558

$0.2970 \quad 0.3051$

381.87

387.06

1.3485

1.3543

0.2885

0.2964

386.85

$\begin{array}{ll}381.65 & 386.85 \\ 1.3470 & 1.3529\end{array}$

$0.2804 \quad 0.2882$

381.42

386.64

0.2728

381.20

1.3442

0.2804

386.42

1.3500
460

480

500

520

0.3931

393.69

1. 3714

0.4029

398.86

1.3769

0.3793

393.49

1.3696

0.3888

398.66

1. 3752

0.3665

393.29

0.3756

398.47

I. 3735

0.3544

393.08

0.3633

398.27

1.3718

0.3431

0.3518

398.08

1.3 .702

I. 3646

0.3325

392.68

1. 3631

0.3409

397.88

1.3687

0.3225

392.47

0.3307

397.69

1.3671

0.3131

392.27

0.3211

397.50

1.3657

0.3042

392.07

0.3120

397.30

1.3642

0.2958

391.86

0.3034

$39 \% .11$

I. 3628

0.2878

391.66

0.295

396.91

I. 3614
0.4125

404.04

I. 3824

0.3981

403.85

1.3806

0.3847

403.67

1. 3789

0.3721

403.48

1.3773

0.3603

403.29

1.3757

0.3493

403.11

1.3742

0.3388

402.92

1.3726

0.3290

402.74

1. 3712

0.3197

40255

1.3697

0.3110

402.37

I. 3683

407.65

402.18

0.3100
407.47

I. 3670

1. 3724

0.4222

409.25

0.4075

409.07

0.3937

408.89

0.3809

408.71

0.3689

408.53

0.3576

408.36

0.3469

408.18

0.3369

408.00

0.3274

407.82

.3752

.3738 


$$
420
$$$$
1.3368
$$

\subsection{6}

380.97

0.2730

$1.3244 \quad 1.3306$

I. 3428

386.21

0.2366

0.2441

800 $365.06 \quad 370.28$

$1.3230 \quad 1.3293$

0.2514

375.51

0.2587

380.75

$1 \cdot 3487$

0.2305

0.2378

1.3354

1.3414

0.2659

386.00

0.2451

375.28

0.2522

1.3473

364.80

370.04

1. 3279

1.3341

380.53

0.2593

1.3401

1.3460

0.2460

$0.2247 \quad 0.2319$

364.53

369.79

0.2390

375.04

1.3328

380.30
1.3388

0.2529

1.3266

1.3447

$\begin{array}{ll}0.2192 & 0.2262 \\ 364.27 & 369.54\end{array}$

$364.27 \quad 369.54$

0.2332

374.81

0.2400

380.08

1.3375

0.2468

385.36

1.3315

\subsection{4}

379.86

0.2410

0.2208

$1.3177 \quad 1.3240$

374.57

1.3302

1.3363

385.15

1.3422

$0.2089 \quad 0.2157$

$363.75 \quad 369.04$

0.2224

374.34

0.2290

379.64

0.2355

384.94

1.3351

1.3410

0.2238

$0.2041 \quad 0.2107$

363.48

1.3152

368.80

0.2173

374.10

I. 3278

379.41
1.3339

0.2302

384.73
1.3398

0.2125

0.1995

363.22

0.2060

1.3139

368.55

1.3203

373.87

0.2188

379.19

0.2251

384.51

1.3327

1.3387

\section{$0.1950 \quad 0.2015$}

362.96

368.30

0.2078

373.64

0.2141

378.97

0.2203

384.30

1. 3315

1.3375

0.1908

362.70

0.1971

368.05

0.2034

373.40

0.2095

0.2156

384.09

1.3304

1.3364

460

480

500

520

0.2803

391.46

1. 3544

0.2875

396.72

1.3601

0.2947

402.00

1.3656

0.2731

391.26

0.2802

396.53

0.2873

401.82

1.3643

0.2653

391.05

1.3518

0.2732

396.34

0.2801

401.63

1.3630

0.2598

390.85

1.3505

0.2666

396.14

0.2733

401.45

1.3618

0.2536

390.65

1.3493

0.2602

395.95

0.2669

401.26

1.3606

0.2476

390.45

0.2542

395.76

0.2607

401.08

I. 3594

0.2420

390.25

1.3469

0.2484

395.57

0.2548

400.90

1.3582

0.2366

390.04

1.3457

0.2429

395.37

0.2491

400.71

1.3570

0.2314

389.84

0.2376

395.18

0.2437

400.53

1.3559

0.2264

389.64

0.2325

394.99

1.3491

0.2385

$400 \cdot 35$

I. 3548

0.2217

389.44

0.2276

394.80

0.2336

400.17

1. 3537
0.3019 407.30

1.3711

0.2943

407.12

1.3698

0.2870

$406 \cdot 94$

1.3685

0.2800

406.77

1. 3673

0.2734

406.59

1.3660

0.2671

406.42

1. 3649

0.2611

406.24

1.3637

0.2553

406.07

1. 3625

0.2498

405.89

$1.361^{4}$

0.2445

405.72

1. 3603

0.2394

405.54

1. 3592 


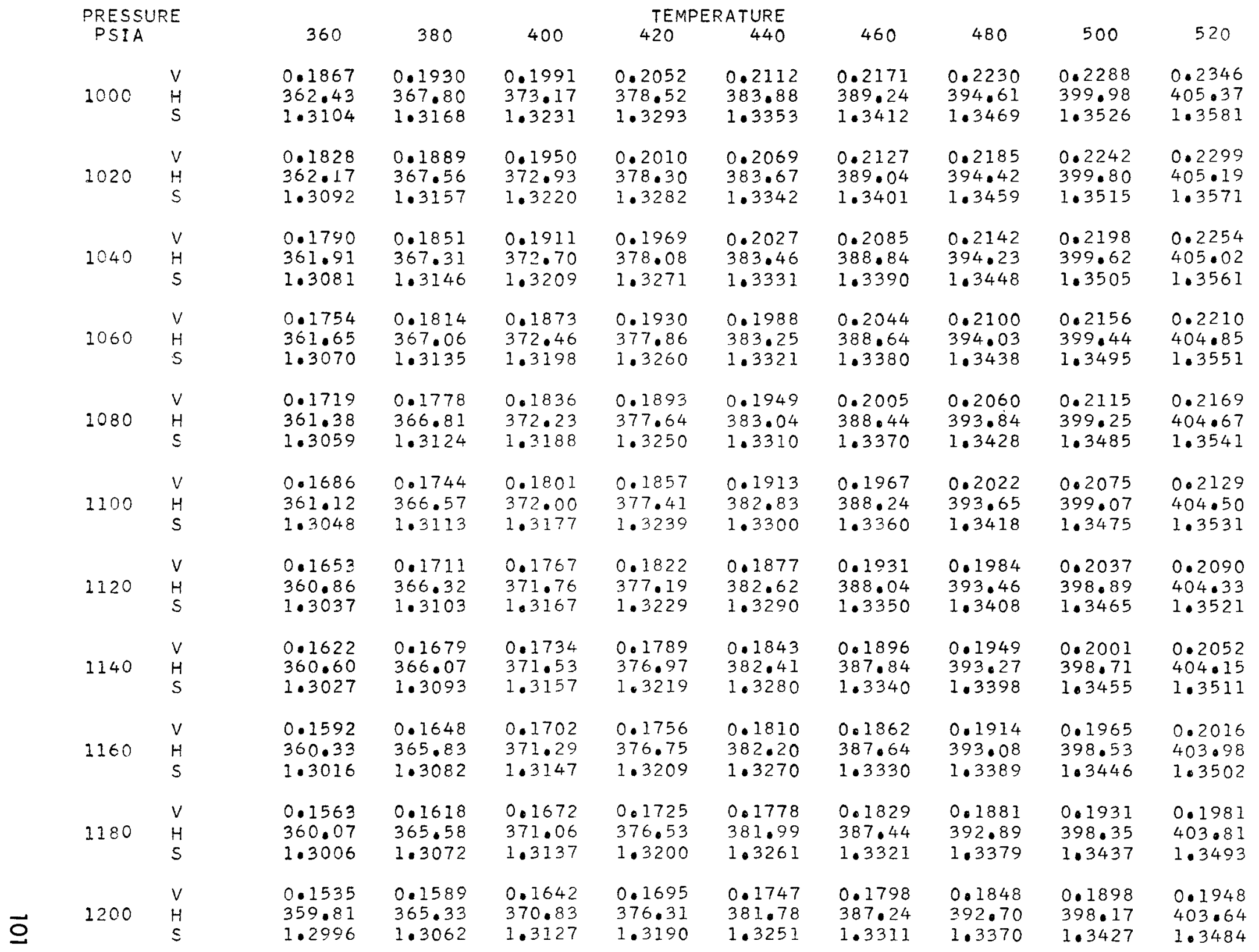




\begin{tabular}{|c|c|c|c|c|c|c|c|c|c|}
\hline 1220 & $\begin{array}{l}0.1508 \\
359.55 \\
1.2986\end{array}$ & $\begin{array}{l}0.1561 \\
365.08 \\
1.3053\end{array}$ & $\begin{array}{l}0.1614 \\
370.60 \\
1.3117\end{array}$ & $\begin{array}{l}0.1666 \\
376.09 \\
1.3180\end{array}$ & $\begin{array}{l}0.1717 \\
381.57 \\
1.3242\end{array}$ & $\begin{array}{l}0.1767 \\
387.04 \\
1.3302\end{array}$ & $\begin{array}{l}0.1817 \\
392.52 \\
1.3361\end{array}$ & $\begin{array}{l}0.1866 \\
397.99 \\
1.3418\end{array}$ & $\begin{array}{l}0.1915 \\
403.46 \\
1.3475\end{array}$ \\
\hline 1240 & $\begin{array}{l}0.1481 \\
359.29 \\
1.2976\end{array}$ & $\begin{array}{l}0.1534 \\
364.84 \\
1.3043\end{array}$ & $\begin{array}{l}0.1586 \\
370.36 \\
1.3108\end{array}$ & $\begin{array}{l}0.1637 \\
375.87 \\
1.3171\end{array}$ & $\begin{array}{l}0.1688 \\
381.36 \\
1.3233\end{array}$ & $\begin{array}{l}0.1737 \\
386.84 \\
1.3293\end{array}$ & $\begin{array}{l}0.1787 \\
392.33 \\
1.3352\end{array}$ & $\begin{array}{l}0.1835 \\
397.81 \\
1.3409\end{array}$ & $\begin{array}{l}0.1883 \\
403.29 \\
1.3466\end{array}$ \\
\hline 260 & $\begin{array}{l}0.1456 \\
359.02 \\
1.2966\end{array}$ & $\begin{array}{l}0.1508 \\
364.59 \\
1.3033\end{array}$ & $\begin{array}{l}0.1559 \\
370.13 \\
1.3098\end{array}$ & $\begin{array}{l}0.1610 \\
375.65 \\
1.3162\end{array}$ & $\begin{array}{l}0.1660 \\
381.15 \\
1.3223\end{array}$ & $\begin{array}{l}0.1709 \\
386.65 \\
1.3284\end{array}$ & $\begin{array}{l}0.1757 \\
392.14 \\
1.3343\end{array}$ & $\begin{array}{l}0.1805 \\
397.63 \\
1.3400\end{array}$ & $\begin{array}{l}0.1853 \\
403.12 \\
1.3457\end{array}$ \\
\hline 80 & $\begin{array}{l}0.1431 \\
358.76 \\
1.2957\end{array}$ & $\begin{array}{l}0.1483 \\
364.35 \\
1.3024\end{array}$ & $\begin{array}{l}0.1534 \\
369.90 \\
1.3089\end{array}$ & $\begin{array}{l}0.1583 \\
375.43 \\
1.3152\end{array}$ & $\begin{array}{l}0.1633 \\
380.94 \\
1.3214\end{array}$ & $\begin{array}{l}0.1681 \\
386.45 \\
1.3275\end{array}$ & $\begin{array}{l}0.1729 \\
391.95 \\
1.3334\end{array}$ & $\begin{array}{l}0.1776 \\
397.45 \\
1.3392\end{array}$ & $\begin{array}{l}0.1823 \\
402.95 \\
1.3448\end{array}$ \\
\hline 0 & $\begin{array}{l}0.1407 \\
358.50 \\
1.2947\end{array}$ & $\begin{array}{l}0.1458 \\
364.10 \\
1.3014\end{array}$ & $\begin{array}{l}0.1508 \\
369.66 \\
1.3080\end{array}$ & $\begin{array}{l}0.1558 \\
375.21 \\
1.3143\end{array}$ & $\begin{array}{l}0.1606 \\
380.73 \\
1.3205\end{array}$ & $\begin{array}{l}0.1654 \\
386.25 \\
1.3266\end{array}$ & $\begin{array}{l}0.1701 \\
391.76 \\
1.3325\end{array}$ & $\begin{array}{l}0.1748 \\
397.27 \\
1.3383\end{array}$ & $\begin{array}{l}0.1795 \\
402.78 \\
1.3440\end{array}$ \\
\hline & $\begin{array}{l}0.1384 \\
358.24 \\
1.2938\end{array}$ & $\begin{array}{l}0.1434 \\
363.85 \\
1.3005\end{array}$ & $\begin{array}{l}0.1484 \\
369.43 \\
1.3071\end{array}$ & $\begin{array}{l}0.1533 \\
374.99 \\
1.3134\end{array}$ & $\begin{array}{l}0.1581 \\
380.53 \\
1.3197\end{array}$ & $\begin{array}{l}0.1628 \\
386.05 \\
1.3257\end{array}$ & $\begin{array}{l}0.1675 \\
391.57 \\
1.3317\end{array}$ & $\begin{array}{l}0.1721 \\
397.09 \\
1.3375\end{array}$ & $\begin{array}{l}0.1767 \\
402.61 \\
1.3431\end{array}$ \\
\hline 40 & $\begin{array}{l}0.1361 \\
357.98 \\
1.2928\end{array}$ & $\begin{array}{l}0.1411 \\
363.61 \\
1.2996\end{array}$ & $\begin{array}{l}0.1460 \\
369.20 \\
1.3062\end{array}$ & $\begin{array}{l}0.1509 \\
374.77 \\
1.3126\end{array}$ & $\begin{array}{l}0.1556 \\
380.32 \\
1.3188\end{array}$ & $\begin{array}{l}0.1603 \\
385.85 \\
1.3249\end{array}$ & $\begin{array}{l}0.1649 \\
391.38 \\
1.3308\end{array}$ & $\begin{array}{l}0.1695 \\
396.91 \\
1.3366\end{array}$ & $\begin{array}{l}0.1740 \\
402.44 \\
1.3423\end{array}$ \\
\hline 360 & $\begin{array}{l}0.1340 \\
357.72 \\
1.2919\end{array}$ & $\begin{array}{l}0.1389 \\
363.36 \\
1.2987\end{array}$ & $\begin{array}{l}0.1437 \\
368.97 \\
1.3053\end{array}$ & $\begin{array}{l}0.1485 \\
374.55 \\
1.3117\end{array}$ & $\begin{array}{l}0.1532 \\
380.11 \\
1.3179\end{array}$ & $\begin{array}{l}0.1578 \\
385.66 \\
1.3240\end{array}$ & $\begin{array}{l}0.1624 \\
391.20 \\
1.3300\end{array}$ & $\begin{array}{l}0.1669 \\
396.73 \\
1.3358\end{array}$ & $\begin{array}{l}0.1714 \\
402.27 \\
1.3415\end{array}$ \\
\hline 380 & $\begin{array}{l}0.1318 \\
357.46 \\
1.2910\end{array}$ & $\begin{array}{l}0.1367 \\
363.12 \\
1.2978\end{array}$ & $\begin{array}{l}0.1415 \\
368.74 \\
1.3044\end{array}$ & $\begin{array}{l}0.1462 \\
374.33 \\
1.3108\end{array}$ & $\begin{array}{r}0.1509 \\
379.90 \\
1.3171\end{array}$ & $\begin{array}{l}0.1554 \\
385.46 \\
1.3232\end{array}$ & $\begin{array}{l}0.1599 \\
391.01 \\
1.3291\end{array}$ & $\begin{array}{l}0.1644 \\
396.55 \\
1.3350\end{array}$ & $\begin{array}{l}0.1688 \\
402.10 \\
1.3407\end{array}$ \\
\hline 100 & $\begin{array}{l}0.1298 \\
357.20 \\
1.2901\end{array}$ & $\begin{array}{l}0.1346 \\
362.87 \\
1.2969\end{array}$ & $\begin{array}{l}0.1394 \\
368.51 \\
1.3035\end{array}$ & $\begin{array}{l}0.1440 \\
374.11 \\
1.3100\end{array}$ & $\begin{array}{l}0.1486 \\
379.70 \\
1.3162\end{array}$ & $\begin{array}{l}0.1531 \\
385.26 \\
1.3223\end{array}$ & $\begin{array}{l}0.1576 \\
390.82 \\
1.3283\end{array}$ & $\begin{array}{l}0.1620 \\
396.37 \\
1.3342\end{array}$ & $\begin{array}{l}0.1663 \\
401.93 \\
1.3399\end{array}$ \\
\hline 50 & $\begin{array}{l}0.1249 \\
356.54 \\
1.2879\end{array}$ & $\begin{array}{l}0.1296 \\
362.26 \\
1.2947\end{array}$ & $\begin{array}{l}0.1342 \\
367.93 \\
1.3014\end{array}$ & $\begin{array}{l}0.1388 \\
373.57 \\
1.3079\end{array}$ & $\begin{array}{l}0.1432 \\
379.18 \\
1.3142\end{array}$ & $\begin{array}{l}0.1476 \\
384.77 \\
1.3203\end{array}$ & $\begin{array}{l}0.1519 \\
390.35 \\
1.3263\end{array}$ & $\begin{array}{l}0.1562 \\
395.93 \\
1.3322\end{array}$ & $\begin{array}{l}0.1605 \\
401.50 \\
1.3379\end{array}$ \\
\hline
\end{tabular}




\begin{tabular}{|c|c|c|c|c|c|c|c|c|c|c|}
\hline \multirow{2}{*}{\multicolumn{2}{|c|}{$\begin{array}{l}\text { PRESSURE } \\
\text { PSIA }\end{array}$}} & \multicolumn{8}{|c|}{ TEMPERATURE } & \multirow[b]{2}{*}{520} \\
\hline & & 360 & 380 & 400 & 420 & 440 & 460 & 480 & 500 & \\
\hline 1500 & $\begin{array}{l}V \\
H \\
S\end{array}$ & $\begin{array}{l}0.1203 \\
355.89 \\
1.2857\end{array}$ & $\begin{array}{l}0.1249 \\
361.65 \\
1.2926\end{array}$ & $\begin{array}{l}0.1294 \\
367.35 \\
1.2993\end{array}$ & $\begin{array}{l}0.1338 \\
373.02 \\
1.3058\end{array}$ & $\begin{array}{l}0.1382 \\
378.66 \\
1.3122\end{array}$ & $\begin{array}{l}0.1425 \\
384.28 \\
1.3183\end{array}$ & $\begin{array}{l}0.1467 \\
389.89 \\
1.3244\end{array}$ & $\begin{array}{l}0.1508 \\
395.49 \\
1.3302\end{array}$ & $\begin{array}{l}0.1550 \\
401.08 \\
1.3360\end{array}$ \\
\hline 1550 & $\begin{array}{l}V \\
H \\
S\end{array}$ & $\begin{array}{l}0.1161 \\
355.25 \\
1.2836\end{array}$ & $\begin{array}{l}0.1205 \\
361.04 \\
1.2906\end{array}$ & $\begin{array}{l}0.1249 \\
366.78 \\
1.2973\end{array}$ & $\begin{array}{l}0.1292 \\
372.48 \\
1.3038\end{array}$ & $\begin{array}{l}0.1335 \\
378.15 \\
1.3102\end{array}$ & $\begin{array}{l}0.1377 \\
383.79 \\
1.3164\end{array}$ & $\begin{array}{l}0.1418 \\
389.42 \\
1.3224\end{array}$ & $\begin{array}{l}0.1458 \\
395.04 \\
1.3284\end{array}$ & $\begin{array}{l}0.1498 \\
400.66 \\
1.3341\end{array}$ \\
\hline 1600 & $\begin{array}{l}V \\
H \\
S\end{array}$ & $\begin{array}{l}0.1121 \\
354.60 \\
1.2815\end{array}$ & $\begin{array}{l}0.1164 \\
360.43 \\
1.2885\end{array}$ & $\begin{array}{l}0.1207 \\
365.20 \\
1.2953\end{array}$ & $\begin{array}{l}0.1249 \\
371.94 \\
1.3019\end{array}$ & $\begin{array}{l}0.1291 \\
377.63 \\
1.3083\end{array}$ & $\begin{array}{l}0.1331 \\
383.31 \\
1.3145\end{array}$ & $\begin{array}{l}0.1372 \\
388.96 \\
1.3206\end{array}$ & $\begin{array}{l}0.1411 \\
394.60 \\
1.3265\end{array}$ & $\begin{array}{l}0.1450 \\
400.24 \\
1.3323\end{array}$ \\
\hline $165 n$ & $\begin{array}{l}V \\
H \\
S\end{array}$ & $\begin{array}{l}0.1083 \\
353.95 \\
1.2795\end{array}$ & $\begin{array}{l}0.1126 \\
359.82 \\
1.2866\end{array}$ & $\begin{array}{l}0.1168 \\
365.63 \\
1.2934\end{array}$ & $\begin{array}{l}0.1209 \\
371.40 \\
1.3000\end{array}$ & $\begin{array}{l}0.1249 \\
377.12 \\
1.3064\end{array}$ & $\begin{array}{l}0.1289 \\
382.82 \\
1.3127\end{array}$ & $\begin{array}{l}0.1328 \\
388.50 \\
1.3188\end{array}$ & $\begin{array}{l}0.1367 \\
394.16 \\
1.3247\end{array}$ & $\begin{array}{l}0.1405 \\
399.82 \\
1.3306\end{array}$ \\
\hline 2700 & $\begin{array}{l}V \\
H \\
S\end{array}$ & $\begin{array}{l}0.1048 \\
353.31 \\
1.2775\end{array}$ & $\begin{array}{l}0.1090 \\
359.22 \\
1.2846\end{array}$ & $\begin{array}{l}0.1131 \\
365.06 \\
1.2915\end{array}$ & $\begin{array}{l}0.1171 \\
370.86 \\
1.2982\end{array}$ & $\begin{array}{l}0.1210 \\
376.61 \\
1.3046\end{array}$ & $\begin{array}{l}0.1249 \\
382.34 \\
1.3109\end{array}$ & $\begin{array}{l}0.1287 \\
388.04 \\
1.3170\end{array}$ & $\begin{array}{l}0.1325 \\
393.73 \\
1.3230\end{array}$ & $\begin{array}{l}0.1362 \\
399.40 \\
1.3288\end{array}$ \\
\hline 1750 & $\begin{array}{l}V \\
H \\
S\end{array}$ & $\begin{array}{l}0.1015 \\
352.67 \\
1.2756\end{array}$ & $\begin{array}{l}0.1056 \\
358.62 \\
1.2827\end{array}$ & $\begin{array}{l}0.1096 \\
364.50 \\
1.2896\end{array}$ & $\begin{array}{l}0.1135 \\
370.32 \\
1.2963\end{array}$ & $\begin{array}{l}0.1174 \\
376.11 \\
1.3028\end{array}$ & $\begin{array}{l}0.1212 \\
381.86 \\
1.3091\end{array}$ & $\begin{array}{l}0.1249 \\
387.58 \\
1.3153\end{array}$ & $\begin{array}{l}0.1286 \\
393.29 \\
1.3213\end{array}$ & $\begin{array}{l}0.1322 \\
398.98 \\
1.3272\end{array}$ \\
\hline 1800 & $\begin{array}{l}V \\
H \\
S\end{array}$ & $\begin{array}{l}0.0983 \\
352.04 \\
1.2737\end{array}$ & $\begin{array}{l}0.1023 \\
358.02 \\
1.2809\end{array}$ & $\begin{array}{l}0.1063 \\
363.93 \\
1.2878\end{array}$ & $\begin{array}{l}0.1101 \\
369.79 \\
1.2946\end{array}$ & $\begin{array}{l}0.1139 \\
375.60 \\
1.3011\end{array}$ & $\begin{array}{l}0.1176 \\
381.38 \\
1.3074\end{array}$ & $\begin{array}{l}0.1213 \\
387.13 \\
1.3136\end{array}$ & $\begin{array}{l}0.1249 \\
392.86 \\
1.3196\end{array}$ & $\begin{array}{l}0.1234 \\
398.57 \\
1.3255\end{array}$ \\
\hline 1850 & $\begin{array}{l}V \\
H \\
S\end{array}$ & $\begin{array}{l}0.0954 \\
351.40 \\
1.2718\end{array}$ & $\begin{array}{l}0.0993 \\
357.42 \\
1.2791\end{array}$ & $\begin{array}{l}0.1032 \\
363.37 \\
1.2861\end{array}$ & $\begin{array}{l}0.1069 \\
369.26 \\
1.2928\end{array}$ & $\begin{array}{l}0.1106 \\
375.10 \\
1.2994\end{array}$ & $\begin{array}{l}0.1143 \\
380.90 \\
1.3057\end{array}$ & $\begin{array}{l}0.1178 \\
386.67 \\
1.3119\end{array}$ & $\begin{array}{l}0.1214 \\
392.42 \\
1.3180\end{array}$ & $\begin{array}{l}0.1249 \\
398.16 \\
1.3239\end{array}$ \\
\hline 1900 & $\begin{array}{l}V \\
H \\
S\end{array}$ & $\begin{array}{l}0.0926 \\
350.77 \\
1.2700\end{array}$ & $\begin{array}{l}0.0964 \\
356.82 \\
1.2773\end{array}$ & $\begin{array}{l}0.1002 \\
362.81 \\
1.2843\end{array}$ & $\begin{array}{l}0.1039 \\
368.73 \\
1.2911\end{array}$ & $\begin{array}{l}0.1075 \\
374.59 \\
1.2977\end{array}$ & $\begin{array}{l}0.1111 \\
380.42 \\
1.3041\end{array}$ & $\begin{array}{l}0.1146 \\
386.22 \\
1.3103\end{array}$ & $\begin{array}{l}0.1181 \\
391.99 \\
1.3164\end{array}$ & $\begin{array}{l}0.1212 \\
397.74 \\
1.3223\end{array}$ \\
\hline 1950 & $\begin{array}{l}V \\
H \\
S\end{array}$ & $\begin{array}{l}0.0899 \\
350.14 \\
1.2682\end{array}$ & $\begin{array}{l}0.0937 \\
356.23 \\
1.2756\end{array}$ & $\begin{array}{l}0.0974 \\
362.25 \\
1.2826\end{array}$ & $\begin{array}{l}0.1010 \\
368.20 \\
1.2895\end{array}$ & $\begin{array}{l}0.1046 \\
374.09 \\
1.2961\end{array}$ & $\begin{array}{l}0.1081 \\
379.95 \\
1.3025\end{array}$ & $\begin{array}{l}0.1115 \\
385.77 \\
1.3087\end{array}$ & $\begin{array}{l}0.1149 \\
391.56 \\
1.3148\end{array}$ & $\begin{array}{l}0.1182 \\
397.33 \\
1.3208\end{array}$ \\
\hline 2000 & $\begin{array}{l}V \\
H \\
S\end{array}$ & $\begin{array}{l}0.0874 \\
349.51 \\
1.2665\end{array}$ & $\begin{array}{l}0.0911 \\
355.65 \\
1.2739\end{array}$ & $\begin{array}{l}0.0947 \\
361.69 \\
1.2810\end{array}$ & $\begin{array}{l}0.0983 \\
367.67 \\
1.2878\end{array}$ & $\begin{array}{l}0.1018 \\
373.60 \\
1.2945\end{array}$ & $\begin{array}{l}0.1052 \\
379.47 \\
1.3009\end{array}$ & $\begin{array}{l}0.1086 \\
385.32 \\
1.3072\end{array}$ & $\begin{array}{l}0.1119 \\
391.13 \\
1.3133\end{array}$ & $\begin{array}{l}0.1152 \\
396.93 \\
1.3193\end{array}$ \\
\hline
\end{tabular}


16.9060

17.2385

17.5709

17.9033

1.5607

424.24
1.5657

429.33
1.5706

434.45
1.5755

439.61
1.5803

18.2357 444.79
1.5851

18.5680

18.9003

19.2326

$35.2225 \quad 15$

$15.5280 \quad 15.8334$

419.17
1.5569

424.23

429.32
1.5668

16.1388

434.44

16.4442

16.7495

1.5898

455.25

460.52

$16 \quad H$

20

12.4219

1.5668

1.5765

444.78

17.0548

1.5990

$419.14 \quad 424.20$

12.6664

12.9109

1.5313

1.5860

$17.3601 \quad 17.6654$

$455.24 \quad 460.51$
$1.5906 \quad 1.5952$

$1.5468 \quad 1.5518$

1.5568

434.41

13.1553
439.57

13.3998

13.6441

13.8885

1.5952

$10.1473 \quad 10.3512$

10.5551
429.26

1.5617

I. 5665

1.5712

1.5759

455.21

14.1328

$10.7590 \quad 10.9628 \quad 11.1666$

11.3704

444.72

449.94

1.5806

460.48

1.5386

1.5436

1.5486

434.38

439.54

1.5630

1.5677

11.5741

11.7778

$455.19 \quad 460.46$

$1.5724 \quad 1.5769$

$\begin{array}{lllll}8.6972 & 8.8721 & 9.0470 & 9.2219 & 9.3967\end{array}$

9.5715

9.7462

$9.9210 \quad 10.0957$

1.5317

1.5367

1.5416

1.5465

1.5513

444.70
1.5561

449.91

9.9210
455.16

$7.6096 \quad 7.7628$

7.9159

8.0690

8.2221

8.3751

419.03

424.10

1.5356

1.5405

439.48

444.57

8.5281

I. 5654

1.5700

6.7638

6.9000

7.0362

7.1724

1.5453

1.5501

449.89

8.6811

455.13

8.8341

1.5548

1.5594

1.5640

$1.5203 \quad 1.5253$

429.16

434.29

7.3085

7.4446

7.5807

7.7168

7.8528

$444 \cdot 64$

449.86

455.11

460.38

1.5400

1.5494

1.5541

1.5587

$6.0871 \quad 6.2098$

6.3324

6.4551

6.5777

6.7002

6.8228

6.9453

7.0678

444.61

449.83

455.08

460.36

1.5352

1.5400

1. 5447

1.5493

1.5539

$5.5334 \quad 5.6450$

5.7566

5.8682

5.9797

6.0912

6.2027

6.3141

6.4255

1.5113

1.5163

1.5212

434.23
1.5261

439.39

444.58

449.80

455.05

460.33

$5.0720 \quad 5.1744$

5.2768

5.3791

5.4814

1.5357

1.5404

1.5450

1.5496

$1.5073 \quad 1.5123$

1.5173

$434 \cdot 20$

439.36

5.5837

5.6859

5.7881

$5.890 ?$

444.55

1.5365

455.03

460.31

$1.5411 \quad 1.5457$

4.6816

418.86

4.7762

4.8708

4.9653

5.0598

5.1542

5.2486

5.3430

5.4374

1.5087

1.5137

434.17

439.33

444.52

449.75

455.00

460.28

I. 5421

1.5375 
PRESSURE PSIA

540

560

4.3470

418.82

1.5003

4.4349

423.89

1.5054

4.0570

418.79

4.1391

423.86

1.5022

1.4972

3.4769

418.70

3.5474

423.78

1.4952

3.0419

418.61

1.4842

3.1037

423.69

1.4892

2.7036

418.53

2.7586

423.61

1.4838

2.4329

418.44

2.4825

423.53

1.4790

2.2114

418.35

2.2566

423.44

1.4747

2.0269

418.26

2.0683

423.36

1.4707

1.8707

418.18

1.9091

423.28

1.4670

1.7369

418.09

1.7725

423.19

1.4636

1.6208

418.00

1.6542

423.11
1.4604

580

4.5227

429.00

1.5103

4.2211

428.97

1.5072

3.6179

428.89

1.5002

3.1654

428.81

1.4941

2.8136

428.73

1. 4888

\subsection{0}

429.64

1.4840

2.3017

428.56

1.4796

2.1098

428.48

1.4757

1.9474

423.40

1.4720

1.8082

428.32

1.4686

1.6875

428.24

1.1.6554

\section{TEMPERATURE}

$600 \quad 620$

4.6106

434.13

4.6983

439.30

1.5152

1.5200

\section{3031}

434.10

1.5121

4.3851

439.27

1.5169

3.6883

434.03

1.5051

3.7587

439.20

1.5099

3.2272

433.95
1.4990

3.2888

439.12

1.5039

$2.8685 \quad 2.9234$

$433.87 \quad 439.05$

1.4937

1.4985

2.5816

433.79

2.6311

438.97

1.4889

1.4937

2.3468

433.72

1.4845

2.3919

438.90

1.4894

2.1512

433.64

2.1925

438.83

1.4854

1.9856

433.56

2.0239

438.75

1.4817

1.8438

1.8793

433.49

1.4735

438.68

1.4784

1.7208

433.41

1.7540

438.60

1.4752
640

660

4.7861
444.50

1.5248

4.4671

444.47

1.5217

3.8290

444.40

1.5147

3.3505

444.33

1.5086

2.9783

444.25

1.5033

2.5805

444.18

1.4985

2.4369

444.11

1.4942

2.2339

444.04

1.4902

\subsection{1}

443.97

1.4865

1.9149

443.90

1.4831

1.7872

443.83

1.4800
4.8738

449.72

1.5295

4.5490

449.69

1.5264

3.8993

449.63

1.5194

3.4121

449.56

1.5133

3.0331

449.49

1.5080

2.7300

449.42

1. 5032

2.4819

449.35

1.4989

2.2752

449.28

1.4949

2.1003

449.22

1.4913

1.9504

449.15

1.4879

1.8204

449.08

1.4847
680

700

4.9615

454.97

1.5341

4.6309

454.95

1.5310

3.9696

454.88

1.5241

3.4737

454.82

1.5180

3.0880

454.75

1.5127

2.7794

454.69

1.5079

2.5269

454.62

1.5035

2.3165

454.55

1.4996

2.1384

454.49

1.4959

2.1766

459.79

$1.9858 \quad 2.0213$

454.42

1.4925

459.73

1.8536

454.36

1.4894

5.0492

1.5387

4.7128

460.23

4. 0399

460.17

3.5353

460.10
1.5226

3.1428

460.04

2.8288

459.98

2.5718

459.92

1. 5081

2.3577 
1.4888

1.5182

1.5477

1.4770

1.4818

I. 4864

459.60

$\begin{array}{llll}422.94 & 428.08 & 433.26 & 438.46\end{array}$

1.5771

1.6064

1.6358

443.69

448.94

1.4694

1.4742

1.4790

$454 \cdot 23$

1.6651

459.54

$1.4597 \quad 1.4646$

1.3502

1.3781

422.86

1.4060

1.4338

429.00
1.4570

433.18

1.4617

1.4895

1.5172 $4 \cdot 38 \cdot 38$

1.4668

443.62
1.4716

448.8

1.4763

1.5450

454.16

1.4810

$1.2789 \quad 1.3054$

417.66

$.3054 \quad 1.3319$

$422.77 \quad 427.92$

1.3583

433.10

1.3847

438.31

1.4111

443.54

1.4375

448.81

1.4638

427.92
1.4545

1.4643

1.4691

1.4739

1.4785

1.3406
443.47

1.3657

1.3155
438.24

1.4619

1.4667

448.74

1.3907

454.03

1.4752 .

1.1041

417.40

1.1271

1.4521

1.4571

1.1959

1.2188

1.2416

$.1501 \quad 1.1730$

438.09

443.33

448.60

1.2644

453.90

1.4718

1.4527

1.4575

1.4523

1.4671

1.1173

1.0962

437.94

443.19

1. 1383

$448 \cdot 47$

1.1592

453.77

1.4678

1.4486

1.4535

1.0314

$0.9337 \quad 0.9533$

417.05

422.19

0.9729

427.37

0.9924

432.57

1.0119

437.80

1.0314
443.05

1.4546

1.0508

448.33

1.0702

1.0702
453.64

1.4640

0.9577

442.91

0.9758

0.9396

1.4463

1. 4511

0.9939

1.4559

1.4606

\title{
0.8770
}

0.8939

442.77

0.9109

448.06

0.9278

453.38

1.4431

1.4479

1.4527

1.4574

0.8381

0.822

437.36

442.63

0.8540

447.93

0.8699

453.25

1.4544

1.4883

1.5727 459.48

1.4856

1.4901 459.41

1.4157 $459 \cdot 35$

1. 4808

1.2872 459.23

1.4764

1.1802 459.10 1.4724

1.0896 458.98

1.4687

1.0119

458.35

1.4652

0.9446 458.73

1.4620

0.8858 458.60 1.4590

416.53
1.4200

421.70
1.4251

426.89
1.4302

1.4352

\begin{abstract}
1.4401
\end{abstract}
1.4497

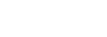


PRESSURE PSIA

540

560

580

600 620

0.7133

416.36

1.4172

0.7284

421.53

I. 4223

0.7436
426.73

426.73
1.4273

0.6878

0.6735

416.19

1.4144

1.4196

0.7022

426.57

1.4246

0.6378

416.01

0.6515

421.20

0.6651

426.41

1.4220

0.6058

415.84

1.4094

0.6188

421.04

1.4145

0.5768

415.67

0.5892

420.87

1.4122

0.5504

415.50

1.4048

0.5623

420.71

1.4099

0.5263

415.33

0.5378

420.54

1.4078

0.5043

415.16

0.5153

420.38

1.4057

0.4840

414.99

1.3986

0.4945

420.22

1.4038

0.4652

414882

0.4754

420.05

1.4019

0.4479

414.65

1.3948

0.4577

419.89

1.4000

\subsection{8}

426.26

1.4196

0.6016

426.10

1.4173

0.5742

425.94

1.4150

0.5492

425.78

1.4129

0.5262

425.63

1.4108

0.5051

425.47
1.4089

0.7587

431.96

1.4323

437.21

0.7165

431.81

I. 4296

0.7308

437.06

$\begin{array}{lll}0.6787 & 0.6923\end{array}$

431.65

1.4270

1.4319

0.6447

0.6577

431.50

1.4246

0.6140

0.6263

431.35
1.4223

0.5860

431.20

436.48

0.5605

431.05

0.5371

430,90

0.5156

430.75

0.4856

425.31

0.4957

430.60

0.4675

1.4120

1.4159

0.4773

0.4871

1.4051

.45

1. 4:102

1.4345

436.92

436.77

1.4295

436.63

1. 4272

0.5979

1.4250

0.5719

436.34

1.4228

0.5480

436.19

1.4208

0.5261

436.05
1.4188

0.5058

435.90

1.4159

435.76

1. 4151

660

680

700

$\begin{array}{lll}0.7888 & 0.8038\end{array}$

I. 4420

447.79

I. 4468

0.8188

453.12

1.4515

0.8338

0.7450

442.35

1.4393

0.7592

447.66

0.7734

453.00

1.4488

0.7058

442.21

0.7193

1.4368

447.53

1.4416

0.6706

442.07

I. 4344

0.6834

447.39

1.4392

0.6387

441.93

0.6505

447.26

1.4368

0.7328

452.87

1.4463

0.6963

452.74

1.4439

0.6632

452.61

1.4416

0.6096

441.79

0.6214

447.12

0.6331

452.48

1.4394

0.5332

441.65

0.5944

0.5944

1.4325

0.6057

452.35

I. 4373

0.5697

0.5589

441.51

1. 4257

446.86

0.5805

452.22

1.4352

0.5365

441.38

0.5574

0.5470

446.72

0.557

452.10
1.4333

0.5159

441.24

1.4218

446.59

I. 4.265

0.5360

451.97

1.4314

0.4968

441.10

0.5065

446.46

1.4248

0.5162

451.84

$\begin{array}{ll}451.84 & 457.25 \\ 1.4296 & 1.4343\end{array}$
1.4562

0.7876

458.35

1.4535

0.7463

458.23

1.42510

0.7091

458.11

1.4485

0.6754 457.98

1.4462

0.6448

457.86

1.4440

0.6169

1457.74

1. 4419

0.5913

457.62

1.430 .

0.5677

7.4330

0.5460

457.37

1.4361

0.5259

1.4343
457.49 


\begin{tabular}{|c|c|c|c|}
\hline \multirow{3}{*}{560} & V & 0.4318 & 0.4413 \\
\hline & H & 414.48 & 419.73 \\
\hline & $s$ & 1.3930 & 1.3982 \\
\hline \multirow{3}{*}{580} & v & 0.4168 & 0.4260 \\
\hline & $H$ & 414.30 & 419.56 \\
\hline & $\mathrm{S}$ & 1.3913 & 1.3965 \\
\hline \multirow{3}{*}{600} & V & 0.4028 & 0.4117 \\
\hline & $H$ & 414.13 & 419.40 \\
\hline & $s$ & 1.3896 & 1.3948 \\
\hline \multirow{3}{*}{620} & v & 0.3897 & 0.3983 \\
\hline & $H$ & 413.97 & 419.24 \\
\hline & $s$ & 1.3880 & 1.3932 \\
\hline \multirow{3}{*}{640} & V & 0.3774 & 0.3858 \\
\hline & $H$ & 413.80 & 419.07 \\
\hline & 5 & 1.3864 & 1.3916 \\
\hline \multirow{3}{*}{660} & V & 0.3658 & 0.3740 \\
\hline & $H$ & 413.63 & 418.91 \\
\hline & $S$ & 1.3849 & 1.3901 \\
\hline \multirow{3}{*}{680} & v & 0.3550 & 0.3630 \\
\hline & $H$ & 413.46 & 418.75 \\
\hline & $s$ & 1.3834 & 1.3886 \\
\hline \multirow{3}{*}{700} & v & 0.3448 & 0.3525 \\
\hline & $\mathrm{H}$ & 413.29 & 418.59 \\
\hline & $s$ & 1.3819 & 1.3872 \\
\hline \multirow{3}{*}{720} & V & 0.3351 & 0.3427 \\
\hline & $H$ & 413.12 & 418.43 \\
\hline & $S$ & 1.3805 & 1.3858 \\
\hline \multirow{3}{*}{740} & v & 0.3259 & 0.3333 \\
\hline & $\mathrm{H}$ & 412.95 & 418.26 \\
\hline & $S$ & 1.3791 & 1.3844 \\
\hline \multirow{3}{*}{760} & v & 0.3172 & 0.3245 \\
\hline & $\begin{array}{l}H \\
S\end{array}$ & $\begin{array}{r}412.78 \\
1.3778\end{array}$ & 418.10 \\
\hline & & $1 \cdot 3778$ & 1.3830 \\
\hline
\end{tabular}

0.4508

\title{
0.4443
}

0.4535

\subsection{6}

0.4717

0.4807 451.59

1.4016

1.4067

1.4116

440.82

446.19

451.59
1.4262

0.4897

\subsection{6}

0.4295

0.4383

424.69
1.4000

430.00

0.4383
435.33

1.4050

1.4100

0.4472

440.68

1.4149

0.4560

446.06

0.4647

451.46

1.4245

0.4070

0.4156

0.4242

424.53

429.85

435.19

0.4328

440.55

0.4413

445.93

0.4498

451.33

1.4182

1.4229

\subsection{2}

0.4026

0.4109

0.4192

440.41

0.4275

445.80

0.4358

451.21

1.4166

1.4214

457.00

1.4309

1.3968

1.4018

1.4068

0.4065

0.3822

0.3904

0.3985

0.4146

0.4226

445.67

451.08

1.4199

0.4735

456.88

1.4292

1.3953

1.4003

1.4053

1.4102

1.4151

0.4103

0.4024

0.3709

0.3788

429.40

0.3867

1.3938

434.76
1.4039

0.3946

440.14

445.53

450.96

0.4583

456.76

1.4276

0.3603

0.3680

0.3757

423.91

429.25

434.61

0.3833

1.3923

1.3974

1.4024

440.00

1.4136

1.4184

0.4440

456.64

1.4261

\subsection{2}

0.3577

0.3652

1.4074

0.3910

445.40

0.3986

450.83

1.4170

0.4306

456.52

1.4246

423.75

429.10

0.3727

1.4122

0.3875

434.47

0.4180

456.40

1.4232

0.3407

0.3480

0.3553

423.60

428.95

434.33

0.4062

456.28

1.4218

0.3317

0.3388

0.3460

423.44

428.81

434.19

450.70

445.27

439.86

1.4156

0.3626

439.73

0.3699

445.14

0.3771

450.58

1.4143

0.3531

0.3531
439.59

0.3602

0.3672

445.01

0.3672
450.45

I. 4130

455.91

0.3949

1.4204

0.3843 456.03 I.4190

1.4033

1.4082

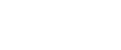

\begin{abstract}
1.4177
\end{abstract}




\begin{tabular}{|c|c|c|c|c|c|c|c|c|c|c|}
\hline \multirow{2}{*}{\multicolumn{2}{|c|}{$\begin{array}{l}\text { PRESSURE } \\
\text { PSIA }\end{array}$}} & \multicolumn{9}{|c|}{ TEMPERATURE } \\
\hline & & 540 & 560 & 580 & 600 & 620 & 640 & 660 & 680 & 700 \\
\hline 780 & $\begin{array}{l}V \\
H \\
S\end{array}$ & $\begin{array}{l}0.3090 \\
412.61 \\
1.3764\end{array}$ & $\begin{array}{l}0.3161 \\
417.94 \\
1.3817\end{array}$ & $\begin{array}{l}0.3231 \\
423.29 \\
1.3869\end{array}$ & $\begin{array}{l}0.3301 \\
428.66 \\
1.3920\end{array}$ & $\begin{array}{l}0.3371 \\
434.05 \\
1.3970\end{array}$ & $\begin{array}{l}0.3 \div 40 \\
439.45 \\
1.4020\end{array}$ & $\begin{array}{l}0.3510 \\
444.88 \\
1.4069\end{array}$ & $\begin{array}{l}0.3578 \\
450.33 \\
1.4117\end{array}$ & $\begin{array}{l}0.3647 \\
455.79 \\
1.4165\end{array}$ \\
\hline 800 & $\begin{array}{l}V \\
H \\
S\end{array}$ & $\begin{array}{l}0.3012 \\
412.44 \\
1.3752\end{array}$ & $\begin{array}{l}0.3081 \\
417.78 \\
1.3804\end{array}$ & $\begin{array}{l}0.3150 \\
423.14 \\
1.3856\end{array}$ & $\begin{array}{l}0.3218 \\
428.51 \\
1.3907\end{array}$ & $\begin{array}{l}0.3287 \\
433.90 \\
1.3958\end{array}$ & $\begin{array}{l}0.3354 \\
439.32 \\
1.4007\end{array}$ & $\begin{array}{l}0.3422 \\
444.75 \\
1.4056\end{array}$ & $\begin{array}{l}0.3489 \\
450.20 \\
1.4105\end{array}$ & $\begin{array}{l}0.3556 \\
455.67 \\
1.4152\end{array}$ \\
\hline 820 & $\begin{array}{l}V \\
H \\
S \\
V \\
H \\
S\end{array}$ & $\begin{array}{l}0.2938 \\
412.27 \\
1.3739 \\
0.2867 \\
412.10 \\
1.3727\end{array}$ & $\begin{array}{l}0.3006 \\
417.62 \\
1.3792 \\
0.2933 \\
417.46 \\
1.3780\end{array}$ & $\begin{array}{l}0.3073 \\
422.98 \\
1.3844 \\
0.2999 \\
422.83 \\
1.3832\end{array}$ & $\begin{array}{l}0.3140 \\
428.36 \\
1.3895 \\
0.3065 \\
423.21 \\
1.3883\end{array}$ & $\begin{array}{l}0.3206 \\
433.76 \\
1.3945 \\
0.3130 \\
433.62 \\
1.3933\end{array}$ & $\begin{array}{l}0.3273 \\
439.18 \\
1.3995 \\
0.3195 \\
439.05 \\
1.3983\end{array}$ & $\begin{array}{l}0.3339 \\
444.62 \\
1.4044 \\
0.3259 \\
444.49 \\
1.4032\end{array}$ & $\begin{array}{l}0.3405 \\
450.08 \\
1.4092 \\
0.3324 \\
449.95 \\
1.4080\end{array}$ & $\begin{array}{l}0.3470 \\
455.55 \\
1.4140 \\
0.3388 \\
455.43 \\
1.4128\end{array}$ \\
\hline 860 & $\begin{array}{l}V \\
H \\
S\end{array}$ & $\begin{array}{l}0.2800 \\
411.94 \\
1.3714\end{array}$ & $\begin{array}{l}0.2865 \\
417.30 \\
1.3767\end{array}$ & $\begin{array}{l}0.2929 \\
422.67 \\
1.3820\end{array}$ & $\begin{array}{l}0.2993 \\
428.07 \\
1.3871\end{array}$ & $\begin{array}{l}0.3057 \\
\angle 33.48 \\
1.3921\end{array}$ & $\begin{array}{l}0.3120 \\
438.91 \\
1.3971\end{array}$ & $\begin{array}{l}0.3184 \\
444.36 \\
1.4020\end{array}$ & $\begin{array}{l}0.3247 \\
449.83 \\
1.4069\end{array}$ & $\begin{array}{l}0.3310 \\
455.31 \\
1.4116\end{array}$ \\
\hline 880 & $\begin{array}{l}V \\
H \\
S\end{array}$ & $\begin{array}{l}0.2735 \\
411.77 \\
1.3703\end{array}$ & $\begin{array}{l}0.2799 \\
417.14 \\
1.3756\end{array}$ & $\begin{array}{l}0.2862 \\
422.52 \\
1.3808\end{array}$ & $\begin{array}{l}0.2925 \\
427.92 \\
1.2859\end{array}$ & $\begin{array}{l}0.2987 \\
433.34 \\
1.3910\end{array}$ & $\begin{array}{l}0.3050 \\
438.77 \\
1.3960\end{array}$ & $\begin{array}{l}0.3112 \\
444.23 \\
j .4009\end{array}$ & $\begin{array}{l}0.3173 \\
449.70 \\
1.4057\end{array}$ & $\begin{array}{l}0.3235 \\
455.19 \\
1.4105\end{array}$ \\
\hline 900 & $\begin{array}{l}V \\
H \\
S\end{array}$ & $\begin{array}{l}0.2674 \\
411.60 \\
1.3691\end{array}$ & $\begin{array}{l}0.2730 \\
416.98 \\
1.3744\end{array}$ & $\begin{array}{l}0.2798 \\
422.37 \\
1.3796\end{array}$ & $\begin{array}{l}0.2860 \\
427.77 \\
1.3848\end{array}$ & $\begin{array}{l}0.2921 \\
433.20 \\
1.3898\end{array}$ & $\begin{array}{l}0.2982 \\
438.64 \\
1.3948\end{array}$ & $\begin{array}{l}0.3043 \\
444.10 \\
1.3998\end{array}$ & $\begin{array}{l}0.3103 \\
449.58 \\
1.4046\end{array}$ & $\begin{array}{l}0.3163 \\
455.08 \\
1.4094\end{array}$ \\
\hline 920 & $\begin{array}{l}V \\
H \\
S\end{array}$ & $\begin{array}{l}0.2615 \\
411.43 \\
1.3680\end{array}$ & $\begin{array}{l}0.2676 \\
416.82 \\
1.3733\end{array}$ & $\begin{array}{l}0.2737 \\
422.21 \\
1.3785\end{array}$ & $\begin{array}{l}0.2797 \\
427.63 \\
1.3837\end{array}$ & $\begin{array}{l}0.2857 \\
433.06 \\
1.3887\end{array}$ & $\begin{array}{l}0.2917 \\
438.50 \\
1.3937\end{array}$ & $\begin{array}{l}0.2977 \\
443.97 \\
1.3986\end{array}$ & $\begin{array}{l}0.3036 \\
449.45 \\
1.4035\end{array}$ & $\begin{array}{l}0.3095 \\
454.96 \\
1.4083\end{array}$ \\
\hline 940 & $\begin{array}{l}V \\
H \\
S\end{array}$ & $\begin{array}{l}0.2558 \\
411.27 \\
1.3668\end{array}$ & $\begin{array}{l}0.2619 \\
416.66 \\
1.3722\end{array}$ & $\begin{array}{l}0.2678 \\
422.06 \\
1.3774\end{array}$ & $\begin{array}{l}0.2737 \\
427.48 \\
1.3826\end{array}$ & $\begin{array}{l}0.2796 \\
432.92 \\
1.3876\end{array}$ & $\begin{array}{l}0.2855 \\
438.37 \\
1.3926\end{array}$ & $\begin{array}{l}0.2913 \\
443.84 \\
1.3976\end{array}$ & $\begin{array}{l}0.2972 \\
449.33 \\
1.4024\end{array}$ & $\begin{array}{l}0.3030 \\
454.84 \\
1.4072\end{array}$ \\
\hline 960 & $\begin{array}{l}V \\
H \\
S\end{array}$ & $\begin{array}{l}0.2504 \\
411.10 \\
1.3657\end{array}$ & $\begin{array}{l}0.2564 \\
416.50 \\
1.3711\end{array}$ & $\begin{array}{l}0.2622 \\
421.91 \\
1.3763\end{array}$ & $\begin{array}{l}0.2680 \\
427.33 \\
1.3815\end{array}$ & $\begin{array}{l}0.2738 \\
432.78 \\
1.3866\end{array}$ & $\begin{array}{l}0.2796 \\
438.24 \\
1.3916\end{array}$ & $\begin{array}{l}0.2853 \\
4.43 .71 \\
1.3965\end{array}$ & $\begin{array}{l}0.2910 \\
449.21 \\
1.4014\end{array}$ & $\begin{array}{l}0.2967 \\
454.72 \\
1.4062\end{array}$ \\
\hline 980 & $\begin{array}{l}V \\
H \\
S\end{array}$ & $\begin{array}{l}0.2453 \\
410.93 \\
1.3647\end{array}$ & $\begin{array}{l}0.2510 \\
416.34 \\
1.3700\end{array}$ & $\begin{array}{l}0.2568 \\
421.76 \\
1.3753\end{array}$ & $\begin{array}{l}0.2625 \\
427.19 \\
1.3804\end{array}$ & $\begin{array}{l}0.2682 \\
432.64 \\
1.3855\end{array}$ & $\begin{array}{l}0.2739 \\
438.10 \\
1.3905\end{array}$ & $\begin{array}{l}0.2795 \\
443.58 \\
1.3955\end{array}$ & $\begin{array}{l}0.2851 \\
449.08 \\
1.4003\end{array}$ & $\begin{array}{l}0.2907 \\
454.60 \\
1.4051\end{array}$ \\
\hline
\end{tabular}




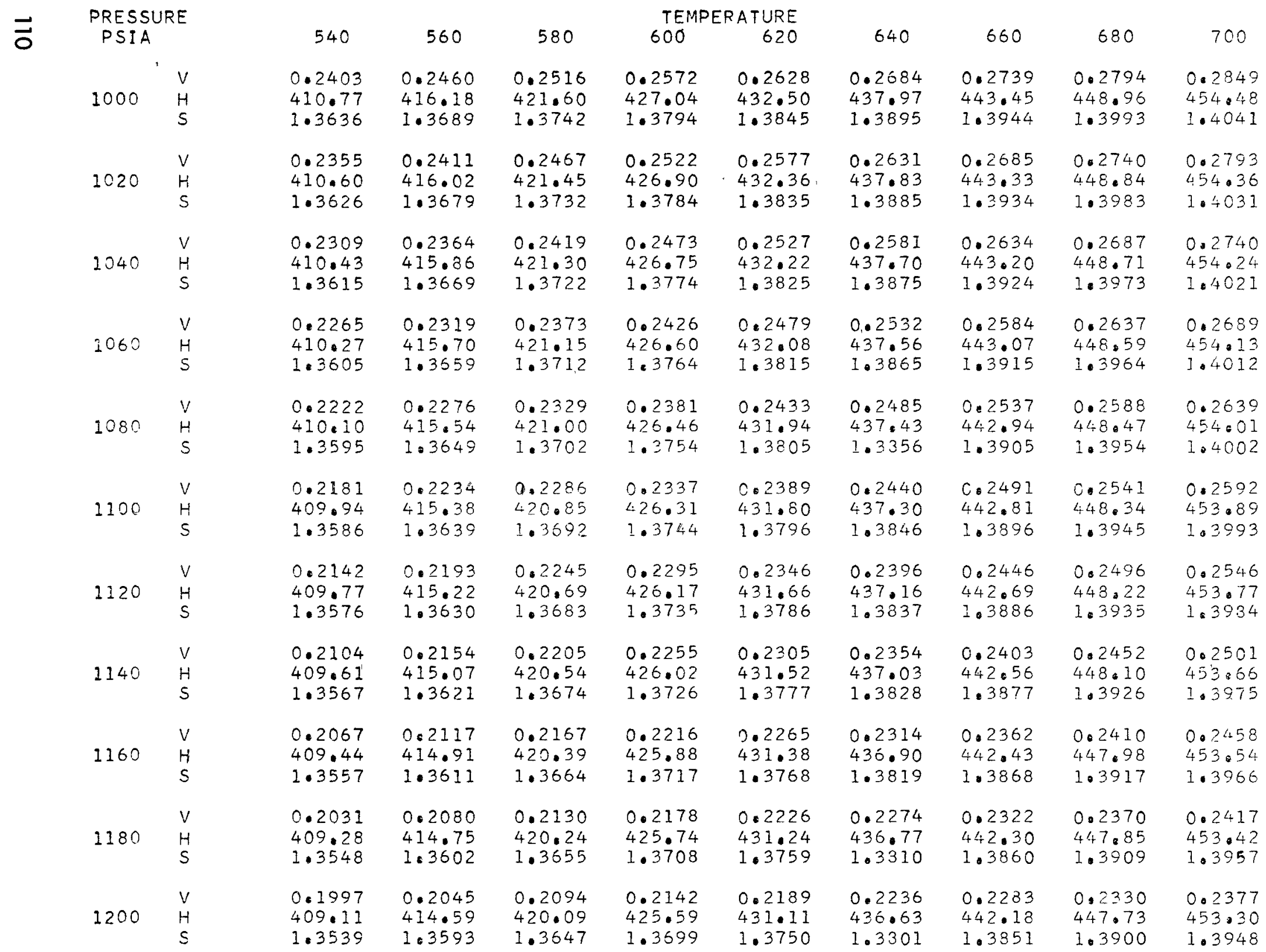




\begin{tabular}{|c|c|c|c|c|c|c|c|c|c|c|}
\hline \multirow{2}{*}{\multicolumn{2}{|c|}{$\begin{array}{l}\text { PRESSURE } \\
\text { PSIA }\end{array}$}} & \multicolumn{9}{|c|}{ TEMPERATURE } \\
\hline & & 540 & 560 & 580 & 600 & 620 & 640 & 660 & 680 & 700 \\
\hline 1220 & $\begin{array}{l}V \\
H \\
S\end{array}$ & $\begin{array}{l}0.1963 \\
408.95 \\
1.3530\end{array}$ & $\begin{array}{l}0.2011 \\
414.44 \\
1.3584\end{array}$ & $\begin{array}{l}0.2059 \\
419.94 \\
1.3638\end{array}$ & $\begin{array}{l}0.2106 \\
425.45 \\
1.3690\end{array}$ & $\begin{array}{l}0.2153 \\
430.97 \\
1.3742\end{array}$ & $\begin{array}{l}0.2200 \\
436.50 \\
1.3792\end{array}$ & $\begin{array}{l}0.2246 \\
442.05 \\
1.3842\end{array}$ & $\begin{array}{l}0.2292 \\
447.61 \\
1.3891\end{array}$ & $\begin{array}{l}0.2338 \\
453.19 \\
1.3940\end{array}$ \\
\hline 1240 & $\begin{array}{l}V \\
H \\
S\end{array}$ & $\begin{array}{l}0.1931 \\
408.78 \\
1.3521\end{array}$ & $\begin{array}{l}0.1978 \\
414.28 \\
1.3576\end{array}$ & $\begin{array}{l}0.2026 \\
419.79 \\
1.3629\end{array}$ & $\begin{array}{l}0.2072 \\
425.30 \\
1.3682\end{array}$ & $\begin{array}{l}0.2118 \\
430.83 \\
1.3733\end{array}$ & $\begin{array}{l}0.2164 \\
436.37 \\
1.3784\end{array}$ & $\begin{array}{l}0.2210 \\
441.92 \\
1.3834\end{array}$ & $\begin{array}{l}0.2255 \\
447.49 \\
1.3883\end{array}$ & $\begin{array}{l}0.2301 \\
453.07 \\
1.3932\end{array}$ \\
\hline 1260 & $\begin{array}{l}V \\
H \\
S\end{array}$ & $\begin{array}{l}0.1900 \\
408.62 \\
1.3513\end{array}$ & $\begin{array}{l}0.1947 \\
414.12 \\
1.3567\end{array}$ & $\begin{array}{l}0.1993 \\
413.64 \\
1.3621\end{array}$ & $\begin{array}{l}0.2039 \\
425.16 \\
1.3673\end{array}$ & $\begin{array}{l}0.2085 \\
430.69 \\
1.3725\end{array}$ & $\begin{array}{l}0.2130 \\
436.24 \\
1.3776\end{array}$ & $\begin{array}{l}0.2175 \\
441.80 \\
1.3826\end{array}$ & $\begin{array}{l}0.2220 \\
447.37 \\
1.3875\end{array}$ & $\begin{array}{l}0.2265 \\
452.95 \\
1.3923\end{array}$ \\
\hline 1280 & $\begin{array}{l}V \\
H \\
S\end{array}$ & $\begin{array}{l}0.1870 \\
408.46 \\
1.3504\end{array}$ & $\begin{array}{l}0.1916 \\
413.97 \\
1.3558\end{array}$ & $\begin{array}{l}0.1962 \\
419.49 \\
1.3612\end{array}$ & $\begin{array}{l}0.2007 \\
425.02 \\
1.3665\end{array}$ & $\begin{array}{l}0.2052 \\
430.56 \\
1.3716\end{array}$ & $\begin{array}{l}0.2097 \\
436.11 \\
1.3767\end{array}$ & $\begin{array}{l}0.2141 \\
441.67 \\
1.3817\end{array}$ & $\begin{array}{l}0.2185 \\
447.25 \\
1.3867\end{array}$ & $\begin{array}{l}0.2229 \\
452.84 \\
1.3915\end{array}$ \\
\hline 1300 & $\begin{array}{l}V \\
H \\
S\end{array}$ & $\begin{array}{l}0.1840 \\
408.29 \\
1.3496\end{array}$ & $\begin{array}{l}0.1886 \\
413.81 \\
1.3550\end{array}$ & $\begin{array}{l}0.1931 \\
419.34 \\
1.3604\end{array}$ & $\begin{array}{l}0.1976 \\
424.87 \\
1.3656\end{array}$ & $\begin{array}{l}0.2020 \\
430.42 \\
1.3708\end{array}$ & $\begin{array}{l}0.2064 \\
435.97 \\
1.3759\end{array}$ & $\begin{array}{l}0.2108 \\
441.54 \\
1.3809\end{array}$ & $\begin{array}{l}0.2152 \\
447.13 \\
1.3859\end{array}$ & $\begin{array}{l}0.2195 \\
452.72 \\
1.3907\end{array}$ \\
\hline 1320 & $\begin{array}{l}V \\
H \\
S\end{array}$ & $\begin{array}{l}0.1812 \\
408.13 \\
1.3487\end{array}$ & $\begin{array}{l}0.1857 \\
413.65 \\
1.3542\end{array}$ & $\begin{array}{l}0.1902 \\
413.19 \\
1.3596\end{array}$ & $\begin{array}{l}0.1946 \\
424.73 \\
1.3648\end{array}$ & $\begin{array}{l}0.1989 \\
430.28 \\
1.3700\end{array}$ & $\begin{array}{l}0.2033 \\
435.84 \\
1.3751\end{array}$ & $\begin{array}{l}0.2076 \\
441.42 \\
1.3801\end{array}$ & $\begin{array}{l}0.2119 \\
447.00 \\
1.3851\end{array}$ & $\begin{array}{l}0.2162 \\
452.61 \\
1.3899\end{array}$ \\
\hline 1340 & $\begin{array}{l}V \\
H \\
S\end{array}$ & $\begin{array}{l}0.1784 \\
407.97 \\
1.3479\end{array}$ & $\begin{array}{l}0.1829 \\
413.50 \\
1.3534\end{array}$ & $\begin{array}{l}0.1873 \\
419.04 \\
1.3587\end{array}$ & $\begin{array}{l}0.1916 \\
424.59 \\
1.3640\end{array}$ & $\begin{array}{l}0.1960 \\
430.14 \\
1.3692\end{array}$ & $\begin{array}{l}0.2003 \\
435.71 \\
1.3743\end{array}$ & $\begin{array}{l}0.2045 \\
441.29 \\
1.3793\end{array}$ & $\begin{array}{l}0.2088 \\
446.88 \\
1.3843\end{array}$ & $\begin{array}{l}0.2130 \\
452.49 \\
1.3891\end{array}$ \\
\hline 1360 & $\begin{array}{l}V \\
H \\
S\end{array}$ & $\begin{array}{l}0.1758 \\
407.80 \\
1.3471\end{array}$ & $\begin{array}{l}0.1801 \\
413.34 \\
1.3526\end{array}$ & $\begin{array}{l}0.1845 \\
418.89 \\
1.3579\end{array}$ & $\begin{array}{l}0.1888 \\
424.45 \\
1.3632\end{array}$ & $\begin{array}{l}0.1931 \\
430.01 \\
1.3684\end{array}$ & $\begin{array}{l}0.1973 \\
435.58 \\
1.3735\end{array}$ & $\begin{array}{l}0.2015 \\
441.17 \\
1.3786\end{array}$ & $\begin{array}{l}0.2057 \\
446.76 \\
1.3835\end{array}$ & $\begin{array}{l}0.2099 \\
452.37 \\
1.3884\end{array}$ \\
\hline 1380 & $\begin{array}{l}V \\
H \\
S\end{array}$ & $\begin{array}{l}0.1732 \\
407.64 \\
1.3463\end{array}$ & $\begin{array}{l}0.1775 \\
413.19 \\
1.3518\end{array}$ & $\begin{array}{l}0.1818 \\
413.74 \\
1.3571\end{array}$ & $\begin{array}{l}0.1860 \\
424.30 \\
1.3624\end{array}$ & $\begin{array}{l}0.1903 \\
429.87 \\
1.3676\end{array}$ & $\begin{array}{l}0.1945 \\
435.45 \\
1.3728\end{array}$ & $\begin{array}{l}0.1986 \\
441.04 \\
1.3778\end{array}$ & $\begin{array}{l}0.2028 \\
446.64 \\
1.3827\end{array}$ & $\begin{array}{l}0.2069 \\
452.26 \\
1.3876\end{array}$ \\
\hline 1400 & $\begin{array}{l}V \\
H \\
S\end{array}$ & $\begin{array}{l}0.1706 \\
407.48 \\
1.3455\end{array}$ & $\begin{array}{l}0.1749 \\
413.03 \\
1.3510\end{array}$ & $\begin{array}{l}0.1792 \\
418.59 \\
1.3564\end{array}$ & $\begin{array}{l}0.1834 \\
424.16 \\
1.3517\end{array}$ & $\begin{array}{l}0.1875 \\
429.73 \\
1.3669\end{array}$ & $\begin{array}{l}0.1917 \\
435.32 \\
1.3720\end{array}$ & $\begin{array}{l}0.1958 \\
440.91 \\
1.3770\end{array}$ & $\begin{array}{l}0.1999 \\
446.52 \\
1.3820\end{array}$ & $\begin{array}{l}0.2040 \\
452.14 \\
1.3869\end{array}$ \\
\hline 1450 & $\begin{array}{l}V \\
H \\
S\end{array}$ & $\begin{array}{l}0.1646 \\
407.07 \\
1.3435\end{array}$ & $\begin{array}{l}0.1688 \\
412.64 \\
1.3490\end{array}$ & $\begin{array}{l}0.1729 \\
418.22 \\
1.3545\end{array}$ & $\begin{array}{l}0.1770 \\
423.81 \\
1.3598\end{array}$ & $\begin{array}{l}0.1810 \\
429.39 \\
1.3650\end{array}$ & $\begin{array}{l}0.1851 \\
434.99 \\
1.3701\end{array}$ & $\begin{array}{l}0.1890 \\
440.60 \\
1.3752\end{array}$ & $\begin{array}{l}0.1930 \\
446.22 \\
1.3801\end{array}$ & $\begin{array}{l}0.1970 \\
451.86 \\
1.3850\end{array}$ \\
\hline
\end{tabular}




PRESSURE
PSIA

$1.3579 \quad 1.3632$

0.1750

0.1789

434.67

0.1828

440.29

0.1866

445.92

0.1904

1.3683

1.3734

1.3783

$0.1577 \quad 0.1616$

0.1655

0.1693

0.1731

0.1769

0.1806 439.98

445.63

0.1843

$\begin{array}{lll}423.10 & 428.72 & 434.34 \\ 1.3561 & 1.3614 & 1.3665\end{array}$

1.3716

1.3766

$451 \cdot 28$

1.3398

$1 \cdot 3453$

1.3508

0.1603

$0.1489 \quad 0.1527$

405.86

411.49

0.1565

1.3380

1.3436

417.12

$422.75 \quad 428.38$

0.1677

434.02

0.1713

439.67

0.1750

1.3596

1.3648

1.3699

$445 \cdot 33$

1.3749

0.1786

$0.1443 \quad 0.1480 \quad 0.1517$

405.46

411.11

416.75

0.1554

422.40

0.1590

428.05

0.1626

433.70

0.1662

439.36

0.1697

445.03

1.3733

$0.1399 \quad 0.1436$

405.07

410.73

0.1472

416.39

0.1507

0.1543

C. 1578

422.05

427.71

433.38

0.1613

439.05

0.1647

$444 \cdot 74$

1. 3716

0.1358

0.1394

0.1429

0.1464

i. 3563

1.3615

1.3666

0.1601

0.1498

0.1533

0.1567

$421.70 \quad 427.38$

1.3329

1.3385

416.02

1.3494

2.3547

433.06

i. 3599

438.75

444.44

1.3701

451.00

1.3798

0.1733

450.71

I. 3782

$0.1320 \quad 0.1354$

0.1389

$0.1423 \quad 0.1457$

$\begin{array}{ll}421.36 & 427.05\end{array}$

0.1490

432.74

1.3584

0.1523

0.1556

438.44

$444 \cdot 15$

1.3585

0.1682

450.43

1.3766

0.1283

0.1317

403.88

409.59

1. 3424

0.1384

$\begin{array}{lll}415.30 & 421.01 & 426.72 \\ 1.3409 & 1.3463 & 1.3516\end{array}$

0.1450

432.42

1.3635

0.1514

0.1482

438.14

443.86

0.1634

450.15

1.3750

I. 3569

1.3620

1.3671

$0.1248 \quad 0.1282$

0.1314

0.1347

0.1379

0.1411

0.1443

426.39

432.11

437.83

0.1475

443.57

1.3656

0.1589

449.87

1.3735

1.3281

1.3393

1.3448

1.3501

1.3554

1.3605

0.13120 .1344

0.1375

0.1406

0.1437

437.53

443.28

1.3642

0.1546

449.58

1.3720

1.3266

408.84

414.58

420.32
1.3433

426.06
1.3487

I. 3539

1. 3591

0.1401

$0.1279 \quad 0.1310$

0.1340

0.1371

437.23

$442 \cdot 99$

1.3628

0.2506

$449 \cdot 30$

1.3706

0.1468

449.03

1.3692

$402.70 \quad 408.47 \quad 414.23$

1.3251

1.3308

1.3419

1.3472

1.3525

0.1431

$448 \cdot 75$

1. 3678 


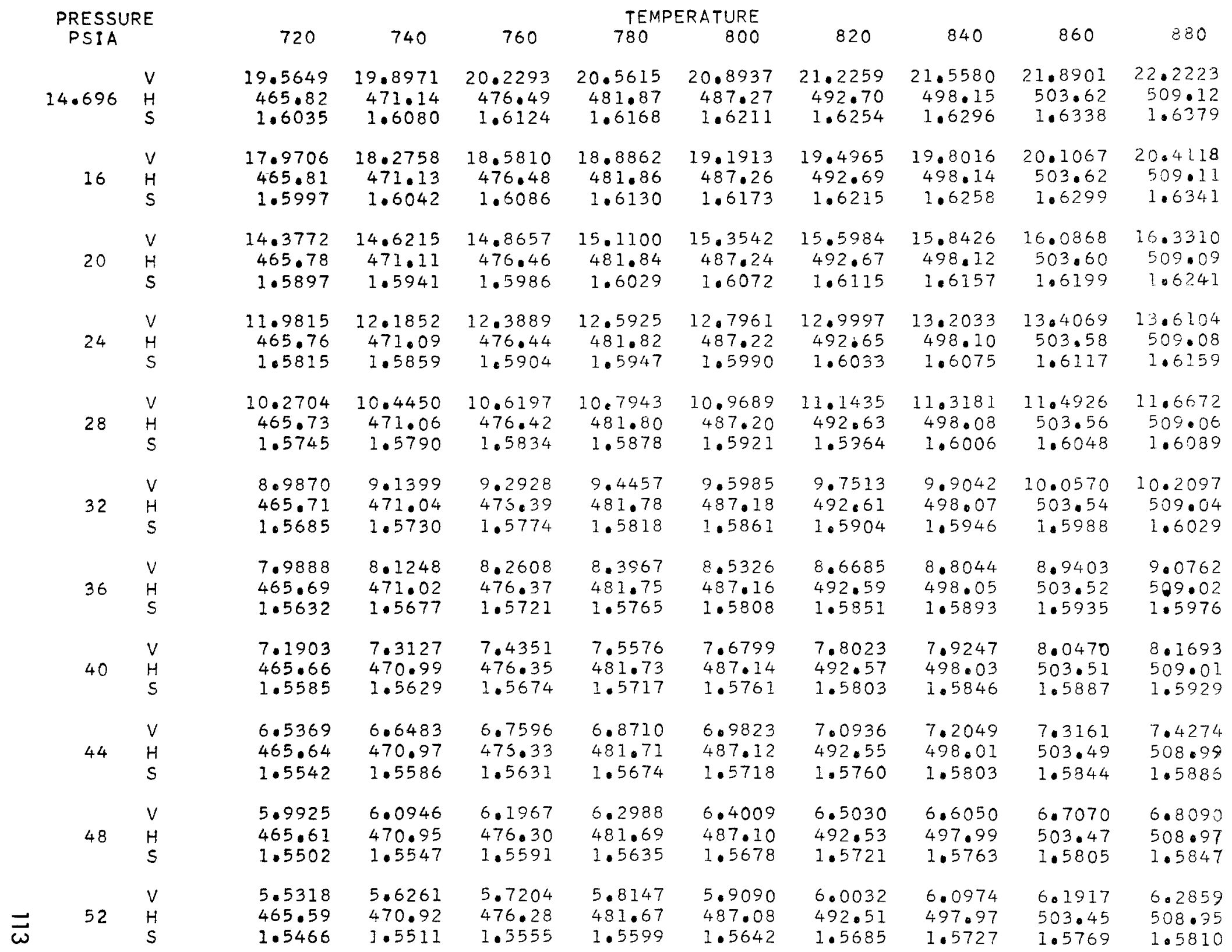




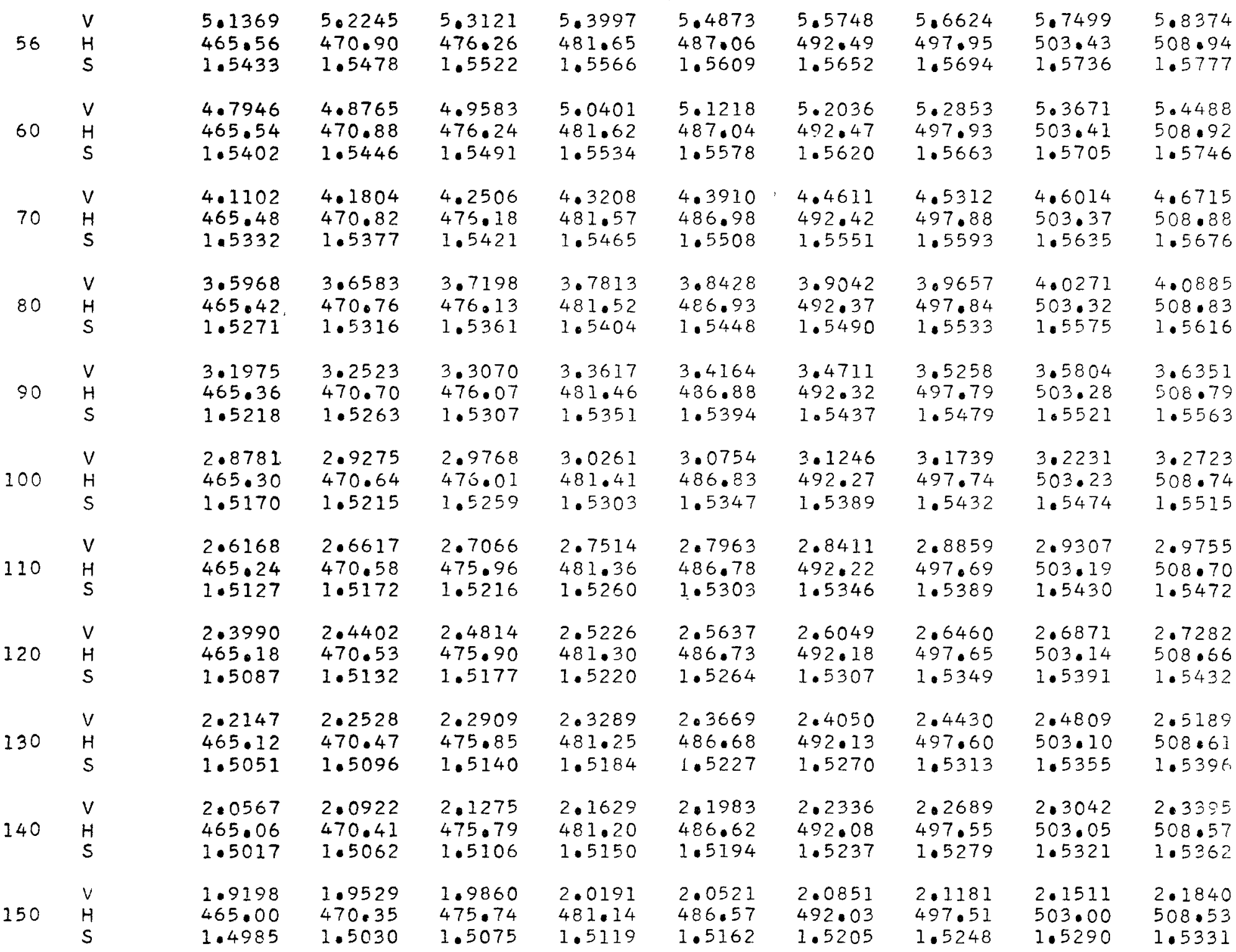




\begin{tabular}{|c|c|c|c|c|c|c|c|c|c|c|}
\hline \multirow{2}{*}{\multicolumn{2}{|c|}{$\begin{array}{l}\text { PRESSURE } \\
\text { PSIA }\end{array}$}} & \multicolumn{8}{|c|}{ TEMPERATURE } & \multirow[b]{2}{*}{880} \\
\hline & & 720 & 740 & 760 & 780 & 800 & 820 & 840 & 860 & \\
\hline 160 & $\begin{array}{l}V \\
H \\
S\end{array}$ & $\begin{array}{l}1.8001 \\
464.94 \\
1.4956\end{array}$ & $\begin{array}{l}1.8311 \\
470.29 \\
1.5001\end{array}$ & $\begin{array}{l}1.3622 \\
475.68 \\
1.5045\end{array}$ & $\begin{array}{l}1.8932 \\
481.09 \\
1.5089\end{array}$ & $\begin{array}{l}1.9242 \\
486.52 \\
1.5133\end{array}$ & $\begin{array}{l}1.9552 \\
491.98 \\
1.5176\end{array}$ & $\begin{array}{l}1.9861 \\
497.46 \\
1.5218\end{array}$ & $\begin{array}{l}2.0171 \\
502.96 \\
1.5260\end{array}$ & $\begin{array}{l}2.0480 \\
508.48 \\
1.5302\end{array}$ \\
\hline 170 & $\begin{array}{l}V \\
H \\
S\end{array}$ & $\begin{array}{l}1.6944 \\
464.87 \\
1.4928\end{array}$ & $\begin{array}{l}1.7236 \\
470.24 \\
1.4973\end{array}$ & $\begin{array}{l}1.7529 \\
475.62 \\
1.5018\end{array}$ & $\begin{array}{l}1.7821 \\
481.04 \\
1.5062\end{array}$ & $\begin{array}{l}1.8113 \\
486.47 \\
1.5105\end{array}$ & $\begin{array}{l}1.8405 \\
491.93 \\
1.5148\end{array}$ & $\begin{array}{l}1.8697 \\
497.41 \\
1.5191\end{array}$ & $\begin{array}{l}1.8988 \\
502.91 \\
1.5233\end{array}$ & $\begin{array}{l}1.9280 \\
508.44 \\
1.5274\end{array}$ \\
\hline 180 & $\begin{array}{l}V \\
H \\
S\end{array}$ & $\begin{array}{l}1.6004 \\
464.81 \\
1.4902\end{array}$ & $\begin{array}{l}1.6281 \\
470.18 \\
1.4947\end{array}$ & $\begin{array}{l}1.6557 \\
475.57 \\
1.4992\end{array}$ & $\begin{array}{l}1.6834 \\
480.98 \\
1.5036\end{array}$ & $\begin{array}{l}1.7110 \\
486.42 \\
1.5079\end{array}$ & $\begin{array}{l}1.7386 \\
491.88 \\
1.5122\end{array}$ & $\begin{array}{l}1.7662 \\
497.36 \\
1.5164\end{array}$ & $\begin{array}{l}1.7937 \\
502.87 \\
1.5206\end{array}$ & $\begin{array}{l}1.8213 \\
508.40 \\
1.5248\end{array}$ \\
\hline 190 & $\begin{array}{l}V \\
H \\
S\end{array}$ & $\begin{array}{l}1.5163 \\
464.75 \\
1.4877\end{array}$ & $\begin{array}{l}1.5426 \\
470.12 \\
1.4922\end{array}$ & $\begin{array}{l}1.5688 \\
475.51 \\
1.4967\end{array}$ & $\begin{array}{l}1.5950 \\
480.93 \\
1.5011\end{array}$ & $\begin{array}{l}1.6212 \\
486.37 \\
1.5054\end{array}$ & $\begin{array}{l}1.6474 \\
491.83 \\
1.5097\end{array}$ & $\begin{array}{l}1.5736 \\
497.32 \\
1.5140\end{array}$ & $\begin{array}{l}1.6997 \\
502.82 \\
1.5182\end{array}$ & $\begin{array}{l}1.7258 \\
508.35 \\
1.5223\end{array}$ \\
\hline 200 & $\begin{array}{l}V \\
H \\
S\end{array}$ & $\begin{array}{l}1.4407 \\
464.69 \\
1.4854\end{array}$ & $\begin{array}{l}1.4657 \\
470.06 \\
1.4899\end{array}$ & $\begin{array}{l}1.4906 \\
475.46 \\
1.4943\end{array}$ & $\begin{array}{l}1.5155 \\
420.88 \\
1.4987\end{array}$ & $\begin{array}{l}1.5404 \\
486.32 \\
1.5031\end{array}$ & $\begin{array}{l}1.5653 \\
491.78 \\
1.5074\end{array}$ & $\begin{array}{l}1.5902 \\
497.27 \\
1.5116\end{array}$ & $\begin{array}{l}1.6151 \\
502.78 \\
1.5158\end{array}$ & $\begin{array}{l}1.6399 \\
508.31 \\
1.5200\end{array}$ \\
\hline 220 & $\begin{array}{l}V \\
H\end{array}$ & $\begin{array}{l}1.3100 \\
464.57\end{array}$ & $\begin{array}{l}1.3328 \\
469.95\end{array}$ & $\begin{array}{l}1.3555 \\
475.35\end{array}$ & $\begin{array}{l}1.3782 \\
480.77\end{array}$ & $\begin{array}{l}1.4009 \\
486.22\end{array}$ & $\begin{array}{l}1.4236 \\
491.68\end{array}$ & $\begin{array}{l}1.4462 \\
497.18\end{array}$ & $\begin{array}{l}1.4689 \\
502.69\end{array}$ & $\begin{array}{l}1.4915 \\
508.22\end{array}$ \\
\hline & $s$ & 1.4810 & 1.4855 & 1.4899 & 1.4943 & 1.4987 & 1.5030 & 1.5073 & 1.5115 & 1.5156 \\
\hline 240 & $\begin{array}{l}V \\
H \\
S\end{array}$ & $\begin{array}{l}1.2011 \\
464.45 \\
1.4770\end{array}$ & $\begin{array}{l}1.2220 \\
469.83 \\
1.4815\end{array}$ & $\begin{array}{l}1.2429 \\
475.24 \\
1.4859\end{array}$ & $\begin{array}{l}1.2638 \\
480.66 \\
1.4903\end{array}$ & $\begin{array}{l}1.2846 \\
486.11 \\
1.4947\end{array}$ & $\begin{array}{l}1.3054 \\
491.59 \\
1.4990\end{array}$ & $\begin{array}{l}1.3262 \\
497.08 \\
1.5033\end{array}$ & $\begin{array}{l}1.3470 \\
502.60 \\
1.5075\end{array}$ & $\begin{array}{l}1.3678 \\
508 \cdot 14 \\
1.5116\end{array}$ \\
\hline 260 & $\begin{array}{l}V \\
H \\
S\end{array}$ & $\begin{array}{l}1.1090 \\
464.33 \\
1.4733\end{array}$ & $\begin{array}{l}1.1283 \\
469.72 \\
1.4778\end{array}$ & $\begin{array}{l}1.1476 \\
475.13 \\
1.4822\end{array}$ & $\begin{array}{l}1.1669 \\
480.56 \\
1.4867\end{array}$ & $\begin{array}{l}1.1862 \\
486.01 \\
1.4910\end{array}$ & $\begin{array}{l}1.2055 \\
491.49 \\
1.4953\end{array}$ & $\begin{array}{l}1.2247 \\
496.99 \\
1.4996\end{array}$ & $\begin{array}{l}1.2439 \\
502.51 \\
1.5038\end{array}$ & $\begin{array}{l}1.2632 \\
508.05 \\
1.5080\end{array}$ \\
\hline 280 & $\begin{array}{l}V \\
H \\
S\end{array}$ & $\begin{array}{l}1.0300 \\
464.22 \\
1.4698\end{array}$ & $\begin{array}{l}1.0480 \\
469.60 \\
1.4743\end{array}$ & $\begin{array}{l}1.0659 \\
475.02 \\
1.4788\end{array}$ & $\begin{array}{l}1.0839 \\
480.45 \\
1.4832\end{array}$ & $\begin{array}{l}1.1019 \\
485.91 \\
1.4876\end{array}$ & $\begin{array}{l}1.1198 \\
491.39 \\
1.4919\end{array}$ & $\begin{array}{l}1.1377 \\
496.89 \\
1.4962\end{array}$ & $\begin{array}{l}1.1556 \\
502.42 \\
1.5004\end{array}$ & $\begin{array}{l}1.1735 \\
507.96 \\
1.5046\end{array}$ \\
\hline 300 & $\begin{array}{l}V \\
H \\
S\end{array}$ & $\begin{array}{l}0.9615 \\
464.10 \\
1.4666\end{array}$ & $\begin{array}{l}0.9783 \\
469.49 \\
1.4711\end{array}$ & $\begin{array}{l}0.9952 \\
474.91 \\
1.4756\end{array}$ & $\begin{array}{l}1.0120 \\
480.35 \\
1.4800\end{array}$ & $\begin{array}{l}1.0288 \\
485.81 \\
1.4844\end{array}$ & $\begin{array}{l}1.0455 \\
491.29 \\
1.4887\end{array}$ & $\begin{array}{l}1.0623 \\
496.80 \\
1.4930\end{array}$ & $\begin{array}{l}1.0790 \\
502.33 \\
1.4972\end{array}$ & $\begin{array}{l}1.0957 \\
507.88 \\
1.5014\end{array}$ \\
\hline 320 & $\begin{array}{l}V \\
H \\
S\end{array}$ & $\begin{array}{l}0.9016 \\
463.98 \\
1.4636\end{array}$ & $\begin{array}{l}0.9174 \\
469.37 \\
1.4681\end{array}$ & $\begin{array}{l}0.9332 \\
474.80 \\
1.4726\end{array}$ & $\begin{array}{l}0.9490 \\
480.24 \\
1.4770\end{array}$ & $\begin{array}{l}0.9648 \\
485.71 \\
1.4814\end{array}$ & $\begin{array}{l}0.9805 \\
491.20 \\
1.4857\end{array}$ & $\begin{array}{l}0.9963 \\
496.71 \\
1.4000\end{array}$ & $\begin{array}{l}1.0120 \\
502.24 \\
1.4942\end{array}$ & $\begin{array}{l}1.0277 \\
507.75 \\
1.4984\end{array}$ \\
\hline
\end{tabular}




\begin{tabular}{|c|c|c|c|c|c|c|c|c|c|c|}
\hline 340 & $\begin{array}{l}V \\
H \\
S\end{array}$ & $\begin{array}{l}0.8488 \\
463.86 \\
1.4608\end{array}$ & $\begin{array}{l}0.8637 \\
469.26 \\
1.4653\end{array}$ & $\begin{array}{l}0.8786 \\
474.69 \\
1.4698\end{array}$ & $\begin{array}{l}0.8935 \\
480.13 \\
1.4742\end{array}$ & $\begin{array}{l}0.9083 \\
485.61 \\
1.4786\end{array}$ & $\begin{array}{l}0.9232 \\
491.10 \\
1.4829\end{array}$ & $\begin{array}{l}0.9380 \\
496.61 \\
1.4872\end{array}$ & $\begin{array}{l}0.952 .9 \\
502.15 \\
1.4914\end{array}$ & $\begin{array}{l}0.9577 \\
507.71 \\
1.4956\end{array}$ \\
\hline 360 & $\begin{array}{l}V \\
H \\
S\end{array}$ & $\begin{array}{l}0.8018 \\
463.74 \\
1.4581\end{array}$ & $\begin{array}{l}0.8159 \\
469.15 \\
1.4626\end{array}$ & $\begin{array}{l}0.8300 \\
474.58 \\
1.4671\end{array}$ & $\begin{array}{l}0.8441 \\
480.03 \\
1.4716\end{array}$ & $\begin{array}{l}0.8582 \\
485.51 \\
1.4759\end{array}$ & $\begin{array}{l}0.8722 \\
491.00 \\
1.4803\end{array}$ & $\begin{array}{l}0.8863 \\
496.52 \\
1.4845\end{array}$ & $\begin{array}{l}0.9003 \\
502.06 \\
1.4888\end{array}$ & $\begin{array}{l}0.9143 \\
507.62 \\
1.4929\end{array}$ \\
\hline 380 & $\begin{array}{l}V \\
H \\
S\end{array}$ & $\begin{array}{l}0.7597 \\
463.62 \\
1.4556\end{array}$ & $\begin{array}{l}0.7731 \\
469.03 \\
1.4601\end{array}$ & $\begin{array}{l}0.7865 \\
474.47 \\
1.4646\end{array}$ & $\begin{array}{l}0.7999 \\
479.92 \\
1.4690\end{array}$ & $\begin{array}{l}0.8133 \\
485.40 \\
1.4734\end{array}$ & $\begin{array}{l}0.8266 \\
490.91 \\
1.4777\end{array}$ & $\begin{array}{l}0.8400 \\
496.43 \\
1.4820\end{array}$ & $\begin{array}{l}0.8533 \\
501.97 \\
1.4863\end{array}$ & $\begin{array}{l}0.8665 \\
507.54 \\
1.4904\end{array}$ \\
\hline 400 & $\begin{array}{l}V \\
H \\
S\end{array}$ & $\begin{array}{l}0.7219 \\
463.50 \\
1.4532\end{array}$ & $\begin{array}{l}0.7347 \\
468.92 \\
1.4577\end{array}$ & $\begin{array}{l}0.7474 \\
474.36 \\
1.4622\end{array}$ & $\begin{array}{l}0.7602 \\
479.82 \\
1.4666\end{array}$ & $\begin{array}{l}0.7729 \\
485.30 \\
1.4710\end{array}$ & $\begin{array}{l}0.7856 \\
490.81 \\
1.4754\end{array}$ & $\begin{array}{l}0.7983 \\
496.34 \\
1.4796\end{array}$ & $\begin{array}{l}0.8109 \\
501.88 \\
1.4839\end{array}$ & $\begin{array}{l}0.8235 \\
507.45 \\
1.4880\end{array}$ \\
\hline 420 & $\begin{array}{l}V \\
H \\
S\end{array}$ & $\begin{array}{l}0.6877 \\
463.38 \\
1.4509\end{array}$ & $\begin{array}{l}0.6999 \\
468.80 \\
1.4554\end{array}$ & $\begin{array}{l}0.7120 \\
474.25 \\
1.4599\end{array}$ & $\begin{array}{l}0.7242 \\
479.71 \\
1.4643\end{array}$ & $\begin{array}{l}0.7363 \\
485.20 \\
1.4687\end{array}$ & $\begin{array}{l}0.7484 \\
490.71 \\
1.4731\end{array}$ & $\begin{array}{l}0.7605 \\
496.24 \\
1.4774\end{array}$ & $\begin{array}{l}0.7726 \\
501.79 \\
1.4816\end{array}$ & $\begin{array}{l}0.7847 \\
507.37 \\
1.4858\end{array}$ \\
\hline 440 & $\begin{array}{l}V \\
H \\
S\end{array}$ & $\begin{array}{l}0.6565 \\
463.26 \\
1.4487\end{array}$ & $\begin{array}{l}0.6682 \\
468.69 \\
1.4532\end{array}$ & $\begin{array}{l}0.6798 \\
47+.14 \\
1.4577\end{array}$ & $\begin{array}{l}0.6915 \\
479.61 \\
1.4622\end{array}$ & $\begin{array}{l}0.7031 \\
485.10 \\
1.4666\end{array}$ & $\begin{array}{l}0.7147 \\
490.62 \\
1.4709\end{array}$ & $\begin{array}{l}0.7263 \\
496.15 \\
1.4752\end{array}$ & $\begin{array}{l}0.7378 \\
501.71 \\
1.4794\end{array}$ & $\begin{array}{l}0.7494 \\
507.28 \\
1.4836\end{array}$ \\
\hline 460 & $\begin{array}{l}V \\
H \\
S\end{array}$ & $\begin{array}{l}0.6281 \\
463.15 \\
1.4466\end{array}$ & $\begin{array}{l}0.6393 \\
468.58 \\
1.4511\end{array}$ & $\begin{array}{l}0.5505 \\
474.03 \\
1.4556\end{array}$ & $\begin{array}{l}0.6616 \\
479.51 \\
1.4601\end{array}$ & $\begin{array}{l}0.5727 \\
485.00 \\
1.4645\end{array}$ & $\begin{array}{l}0.5338 \\
490.52 \\
1.4688\end{array}$ & $\begin{array}{l}0.5949 \\
496.06 \\
1.4731\end{array}$ & $\begin{array}{l}0.7060 \\
501.62 \\
1.4773\end{array}$ & $\begin{array}{l}0.7170 \\
507.19 \\
1.4815\end{array}$ \\
\hline 480 & $\begin{array}{l}V \\
H \\
S\end{array}$ & $\begin{array}{l}0.6021 \\
463.03 \\
1.4445\end{array}$ & $\begin{array}{l}0.6128 \\
468.46 \\
1.4491\end{array}$ & $\begin{array}{l}0.6235 \\
473.92 \\
1.4536\end{array}$ & $\begin{array}{l}0.6342 \\
479.40 \\
1.4581\end{array}$ & $\begin{array}{l}0.6449 \\
484.90 \\
1.4625\end{array}$ & $\begin{array}{l}0.6556 \\
490.42 \\
1.4668\end{array}$ & $\begin{array}{l}0.6662 \\
495.97 \\
1.4711\end{array}$ & $\begin{array}{l}0.6769 \\
501.53 \\
1.4753\end{array}$ & $\begin{array}{l}0.6874 \\
507.10 \\
1.4795\end{array}$ \\
\hline 500 & $\begin{array}{l}V \\
H \\
S\end{array}$ & $\begin{array}{l}0.5781 \\
462.91 \\
1.4426\end{array}$ & $\begin{array}{l}0.5884 \\
468.35 \\
1.4472\end{array}$ & $\begin{array}{l}0.5988 \\
473.81 \\
1.4517\end{array}$ & $\begin{array}{l}0.6090 \\
479.30 \\
1.4561\end{array}$ & $\begin{array}{l}0.6193 \\
484.80 \\
1.4605\end{array}$ & $\begin{array}{l}0.6296 \\
490.33 \\
1.4649\end{array}$ & $\begin{array}{l}0.6398 \\
495.87 \\
1.4692\end{array}$ & $\begin{array}{l}0.6500 \\
501.43 \\
1.4734\end{array}$ & $\begin{array}{l}0.6602 \\
507.02 \\
1.4776\end{array}$ \\
\hline 520 & $\begin{array}{l}V \\
H \\
S\end{array}$ & $\begin{array}{l}0.5560 \\
462.79 \\
1.4407\end{array}$ & $\begin{array}{l}0.5659 \\
468.24 \\
1.4453\end{array}$ & $\begin{array}{l}0.5759 \\
473.71 \\
1.4498\end{array}$ & $\begin{array}{l}0.5858 \\
479.19 \\
1.4543\end{array}$ & $\begin{array}{l}0.5957 \\
484.70 \\
1.4587\end{array}$ & $\begin{array}{l}0.6056 \\
490.23 \\
1.4630\end{array}$ & $\begin{array}{l}0.6154 \\
495.78 \\
1.4673\end{array}$ & $\begin{array}{l}0.6252 \\
501.35 \\
1.4716\end{array}$ & $\begin{array}{l}0.6351 \\
506.93 \\
1.4758\end{array}$ \\
\hline 540 & $\begin{array}{l}V \\
H \\
S\end{array}$ & $\begin{array}{l}0.5355 \\
462.68 \\
1.4389\end{array}$ & $\begin{array}{l}0.5451 \\
468.13 \\
1.4435\end{array}$ & $\begin{array}{l}0.5547 \\
473.60 \\
1.4480\end{array}$ & $\begin{array}{l}0.5643 \\
479.09 \\
1.4525\end{array}$ & $\begin{array}{l}0.5738 \\
484.60 \\
1.4569\end{array}$ & $\begin{array}{l}0.5834 \\
490.14 \\
1.4612\end{array}$ & $\begin{array}{l}0.5928 \\
495.68 \\
1.4655\end{array}$ & $\begin{array}{l}0.6023 \\
501.26 \\
1.4698\end{array}$ & $\begin{array}{l}0.6118 \\
506.85 \\
1.4740\end{array}$ \\
\hline
\end{tabular}




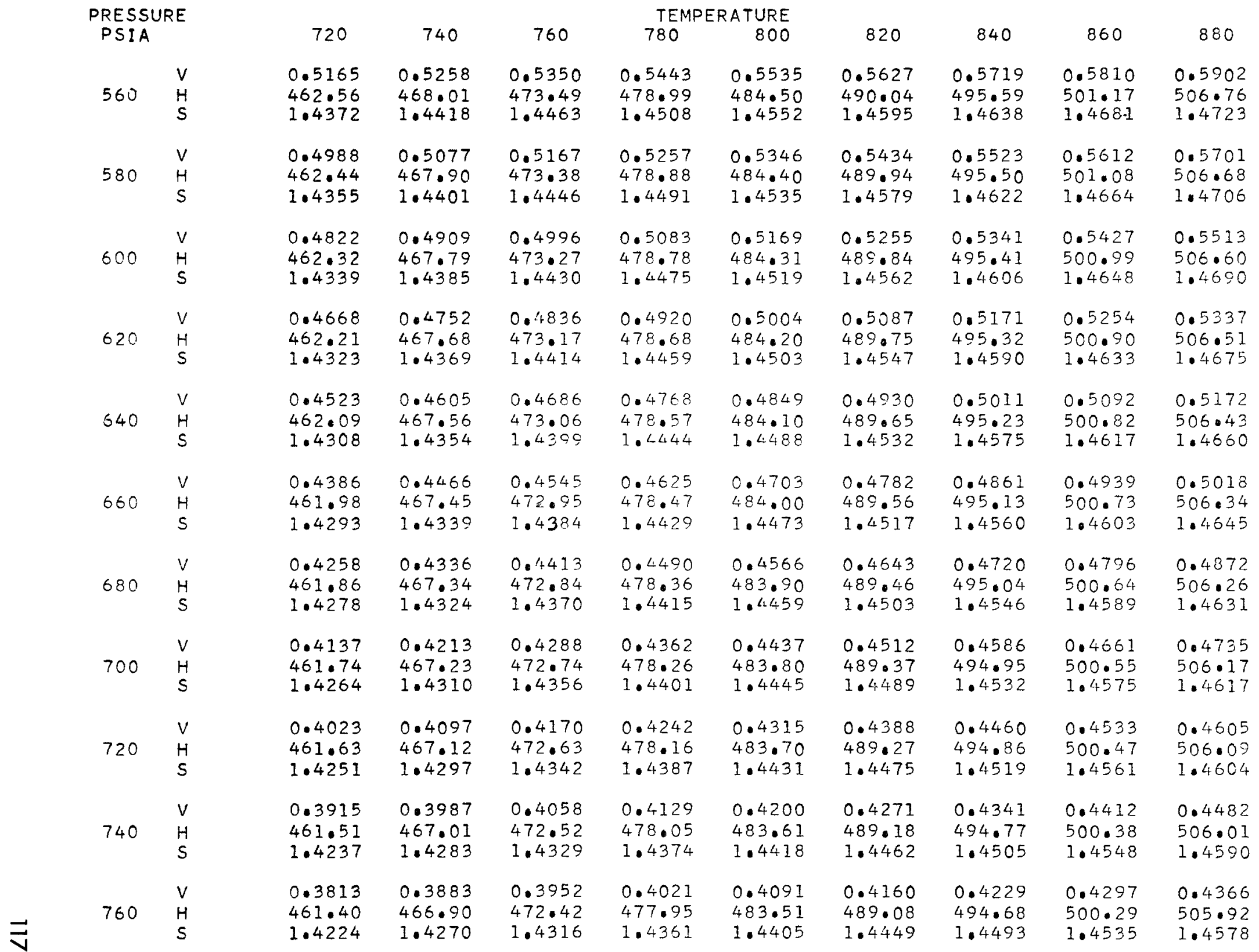




\section{PRESSURE
$\bar{\infty}$
PSIA}

780

800

820

840

860

880

900

920

$940 \quad H$

960

980

720

740

760

780

EMPERATURE

0.3716

461.28

1.4211

0.3784

466.79

1.4258

0.3623

$4 E 1.16$

1.4199

0.3690

466.68

1.4245

0.3536

461.05

0.3601

466.56

1.4233

0.3452

460.93

1.4175

0.3516

466.45

1.4221

0.3372

460.82

1.4163

0.3435

466.34

1.4210

0.3296

460.71

0.3357

466.23

1.4198

0.3224

460.59

0.3283

466.12

1.4187

0.3154

460.48

1.4130

0.3212

466.01

1.4176

0.3087

460.36

1.4119

0.3145

465.90

1.4166

0.3024

460.25

0.3080

465.79

1.4155

0.2962

460.13

1.4098

0.3017

1.4145
0.3852

472.31

1.4303

0.3756

472.20

1.4291

\subsection{5}

472.09

1.4279

0.3579

471.99

1.4267

0.3497

471.88

1.4255

0.3418

471.77

1.4244

0.3343

471.67

1.4233

0.3271

471.56

1.4222

0.3202

471.46

1.4212

0.3136

471.35

1.4201

0.3073

471.25

1.4191

\subsection{9 \\ 477.85}

1.4348

477.75

1.4336

0.3730

477.64

1.4324

0.3642

477.54

1.4312

0.3558

477.44

1.4301

0.3478

477.34

1.4289

0.3402

477.24

1.4278

0.3329

477.13

1.4268

0.3259

477.03

1.4257

0.3192

476.93

1.4247

0.3128

476.83

1.4236
0.3822
800

0.3987

483.41

1.4393

0.3888

483.31

1.4380

0.3795

483.21

1.4368

0.3705

483.12

1.4357

0.3620

483.02

1.4345

0.3539

482.92

1.4334

0.3461

482.82

1.4323

0.3387

482.72

1.4312

0.3316

482.63

1.4302

0.3248

482.53

1.4291

0.3182

482.43

1.4281
820

840

860

880

0.4054

488.99

1.4437

0.3954

488.90

1.4424

0.3859

488.80

1.4412

0.3768

488.71

1.4401

0.3682

488.61

1.4389

0.3599

488.52

1.4378

0.3520

488.43

1.4367

0.3445

488.33

1.4356

0.3373

488.24

1.4346

0.3303

488.14

1.4335

0.3237

488.05

1.4325
0.4122

494.59

1.4480

0.4020

494.50

1.4468

0.3923

494.41

1.4456

0.3831

494.32

1.4444

0.3743

494.23

1.4433

0.3659

494.14

1.4422

0.3579

494.05

1.4411

0.3503

493.96

1.4400

0.3429

493.87

1.4389

0.3359

493.78

1.4379

0.3291

493.69

1.4369
0.4189

500.20

1.4523

0.4085

500.12

1.4511

0.3987

500.03

1.4499

0.3894

$+99.94$

1.4487

0.3805

499.86

1.4476

0.3719

499.77

1.4465

0.3638

499.68

1.4454

0.3560

499.60

1.4443

0.3486

499.51

1.4432

0.3414

499.43

1.4422

0.3346

499.34 


\begin{tabular}{|c|c|c|c|c|c|c|c|c|c|c|}
\hline \multirow{2}{*}{\multicolumn{2}{|c|}{$\begin{array}{l}\text { PRESSURE } \\
\text { PSIA }\end{array}$}} & \multicolumn{8}{|c|}{ TEMPERATURE } & \multirow{2}{*}{880} \\
\hline & & 720 & 740 & 760 & 780 & 800 & 820 & 840 & 860 & \\
\hline 1000 & $\begin{array}{l}V \\
H \\
S\end{array}$ & $\begin{array}{l}0.2904 \\
460.02 \\
1.4088\end{array}$ & $\begin{array}{l}0.2958 \\
465.57 \\
1.4135\end{array}$ & $\begin{array}{l}0.3012 \\
471.14 \\
1.4181\end{array}$ & $\begin{array}{l}0.3066 \\
476.73 \\
1.4226\end{array}$ & $\begin{array}{l}0.3120 \\
482.34 \\
1.4271\end{array}$ & $\begin{array}{l}0.3173 \\
487.96 \\
1.4315\end{array}$ & $\begin{array}{l}0.3227 \\
493.60 \\
1.4359\end{array}$ & $\begin{array}{l}0.3280 \\
499.25 \\
1.4402\end{array}$ & $\begin{array}{l}0.3333 \\
504.93 \\
1.4445\end{array}$ \\
\hline 1020 & $\begin{array}{l}V \\
H \\
S\end{array}$ & $\begin{array}{l}0.2847 \\
459.91 \\
1.4078\end{array}$ & $\begin{array}{l}0.2900 \\
465.46 \\
1.4125\end{array}$ & $\begin{array}{l}0.2953 \\
471.04 \\
1.4171\end{array}$ & $\begin{array}{l}0.3006 \\
476.63 \\
1.4217\end{array}$ & $\begin{array}{l}0.3059 \\
482.24 \\
1.4261\end{array}$ & $\begin{array}{l}0.3112 \\
487.87 \\
1.4306\end{array}$ & $\begin{array}{l}0.3164 \\
493.51 \\
1.4349\end{array}$ & $\begin{array}{l}0.3217 \\
499.17 \\
1.4393\end{array}$ & $\begin{array}{l}0.3269 \\
504.84 \\
1.4435\end{array}$ \\
\hline 1040 & $\begin{array}{l}V \\
H \\
S\end{array}$ & $\begin{array}{l}0.2793 \\
459.79 \\
1.4069\end{array}$ & $\begin{array}{l}0.2845 \\
465.35 \\
1.4115\end{array}$ & $\begin{array}{l}0.2897 \\
470.93 \\
1.4161\end{array}$ & $\begin{array}{l}0.2949 \\
476.53 \\
1.4207\end{array}$ & $\begin{array}{l}0.3001 \\
482.14 \\
1.4252\end{array}$ & $\begin{array}{l}0.3053 \\
487.77 \\
1.4296\end{array}$ & $\begin{array}{l}0.3104 \\
493.42 \\
1.4340\end{array}$ & $\begin{array}{l}0.3156 \\
499.08 \\
1.4383\end{array}$ & $\begin{array}{l}0.3207 \\
504.76 \\
1.4426\end{array}$ \\
\hline 1060 & $\begin{array}{l}V \\
H \\
S\end{array}$ & $\begin{array}{l}0.2741 \\
459.68 \\
1.4059\end{array}$ & $\begin{array}{l}0.2792 \\
465.24 \\
1.4106\end{array}$ & $\begin{array}{l}0.2843 \\
470.83 \\
1.4152\end{array}$ & $\begin{array}{l}0.2894 \\
476.43 \\
1.4197\end{array}$ & $\begin{array}{l}0.2945 \\
482.05 \\
1.4242\end{array}$ & $\begin{array}{l}0.2996 \\
487.68 \\
1.4287\end{array}$ & $\begin{array}{l}0.3047 \\
493.33 \\
1.4330\end{array}$ & $\begin{array}{l}0.3097 \\
499.00 \\
1.4374\end{array}$ & $\begin{array}{l}0.3148 \\
504.68 \\
1.4416\end{array}$ \\
\hline 1080 & $\begin{array}{l}V \\
H \\
S\end{array}$ & $\begin{array}{l}0.2690 \\
459.57 \\
1.4050\end{array}$ & $\begin{array}{l}0.2741 \\
465.13 \\
1.4096\end{array}$ & $\begin{array}{l}0.2791 \\
470.72 \\
1.4143\end{array}$ & $\begin{array}{l}0.2841 \\
476.33 \\
1.4188\end{array}$ & $\begin{array}{l}0.2892 \\
481.95 \\
1.4233\end{array}$ & $\begin{array}{l}0.2942 \\
487.59 \\
1.4277\end{array}$ & $\begin{array}{l}0.2991 \\
493.24 \\
1.4321\end{array}$ & $\begin{array}{l}0.3041 \\
498.91 \\
1.4364\end{array}$ & $\begin{array}{l}0.3091 \\
504.60 \\
1.4407\end{array}$ \\
\hline 1100 & $\begin{array}{l}V \\
H \\
S\end{array}$ & $\begin{array}{l}0.2642 \\
459.45 \\
1.4040\end{array}$ & $\begin{array}{l}0.2691 \\
465.03 \\
1.4087\end{array}$ & $\begin{array}{l}0.2741 \\
470.62 \\
1.4133\end{array}$ & $\begin{array}{l}0.2790 \\
476.23 \\
1.4179\end{array}$ & $\begin{array}{l}0.2840 \\
481.85 \\
1.4224\end{array}$ & $\begin{array}{l}0.2889 \\
487.49 \\
1.4268\end{array}$ & $\begin{array}{l}0.2938 \\
493.15 \\
1.4312\end{array}$ & $\begin{array}{l}0.2987 \\
498.83 \\
1.4355\end{array}$ & $\begin{array}{l}0.3035 \\
504.51 \\
1.4398\end{array}$ \\
\hline 1120 & $\begin{array}{l}V \\
H \\
S\end{array}$ & $\begin{array}{l}0.2595 \\
450.34 \\
1.4031\end{array}$ & $\begin{array}{l}0.2644 \\
464.92 \\
1.4078\end{array}$ & $\begin{array}{l}0.2693 \\
470.51 \\
1.4124\end{array}$ & $\begin{array}{l}0.2741 \\
476.13 \\
1.4170\end{array}$ & $\begin{array}{l}0.2790 \\
481.76 \\
1.4215\end{array}$ & $\begin{array}{l}0.2338 \\
487.40 \\
1.4259\end{array}$ & $\begin{array}{l}0.2886 \\
493.06 \\
1.4303\end{array}$ & $\begin{array}{l}0.2934 \\
498.74 \\
1.4346\end{array}$ & $\begin{array}{l}0.2982 \\
504.43 \\
1.4389\end{array}$ \\
\hline 1140 & $\begin{array}{l}V \\
H \\
S\end{array}$ & $\begin{array}{l}0.2550 \\
459.23 \\
1.4022\end{array}$ & $\begin{array}{l}0.2598 \\
464.81 \\
1.4069\end{array}$ & $\begin{array}{l}0.2646 \\
470.41 \\
1.4115\end{array}$ & $\begin{array}{l}0.2694 \\
476.03 \\
1.4161\end{array}$ & $\begin{array}{l}0.2742 \\
481.66 \\
1.4206\end{array}$ & $\begin{array}{l}0.2789 \\
487.31 \\
1.4250\end{array}$ & $\begin{array}{l}0.2837 \\
492.97 \\
1.4294\end{array}$ & $\begin{array}{l}0.2884 \\
498.65 \\
1.4338\end{array}$ & $\begin{array}{l}0.2931 \\
504.35 \\
1.4380\end{array}$ \\
\hline 1160 & $\begin{array}{l}V \\
H \\
S\end{array}$ & $\begin{array}{l}0.2506 \\
459.12 \\
1.4013\end{array}$ & $\begin{array}{l}0.2553 \\
464.70 \\
2.4060\end{array}$ & $\begin{array}{l}0.2601 \\
470.31 \\
1.4107\end{array}$ & $\begin{array}{l}0.2648 \\
475.93 \\
1.4152\end{array}$ & $\begin{array}{l}0.2695 \\
481.56 \\
1.4197\end{array}$ & $\begin{array}{l}0.2742 \\
487.22 \\
1.4242\end{array}$ & $\begin{array}{l}0.2788 \\
492.89 \\
1.4286\end{array}$ & $\begin{array}{l}0.2835 \\
498.57 \\
1.4329\end{array}$ & $\begin{array}{l}0.2831 \\
504.27 \\
1.4372\end{array}$ \\
\hline 1180 & $\begin{array}{l}V \\
H \\
S\end{array}$ & $\begin{array}{l}0.2464 \\
459.00 \\
1.4005\end{array}$ & $\begin{array}{l}0.2511 \\
464.59 \\
1.4052\end{array}$ & $\begin{array}{l}0.2557 \\
470.20 \\
1.4098\end{array}$ & $\begin{array}{l}0.2604 \\
475.83 \\
1.4144\end{array}$ & $\begin{array}{l}0.2650 \\
481.47 \\
1.4189\end{array}$ & $\begin{array}{l}0.2696 \\
487.13 \\
1.4233\end{array}$ & $\begin{array}{l}0.2742 \\
492.80 \\
1.4277\end{array}$ & $\begin{array}{l}0.2788 \\
498.48 \\
1.4321\end{array}$ & $\begin{array}{l}0.2833 \\
504.19 \\
1.4363\end{array}$ \\
\hline 1200 & $\begin{array}{l}V \\
H \\
S\end{array}$ & $\begin{array}{l}0.2423 \\
458.89 \\
1.3996\end{array}$ & $\begin{array}{l}0.2469 \\
464.48 \\
1.4043\end{array}$ & $\begin{array}{l}0.2515 \\
470.10 \\
1.4089\end{array}$ & $\begin{array}{l}0.2561 \\
475.73 \\
1.4135\end{array}$ & $\begin{array}{l}0.2606 \\
481.37 \\
1.4180\end{array}$ & $\begin{array}{l}0.2652 \\
487.03 \\
1.4225\end{array}$ & $\begin{array}{l}0.2697 \\
492.71 \\
1.4269\end{array}$ & $\begin{array}{l}0.2742 \\
498.40 \\
1.4312\end{array}$ & $\begin{array}{l}0.2787 \\
504.11 \\
1.4355\end{array}$ \\
\hline
\end{tabular}


0.2474 780

0.2346 458.66

0.2390

0.2520

475.63

0.2564 1.4081

1.4127

481.28

0.2435 464.27

1.4026

469.89

0.2479

1.4172

475.53
1.4119

0.2524

481.18

1.4164

0.2309

1260

458.55

0.2353

464.16

1.4018

0.2397
469.79

1.4065

0.2441

475.43

0.2484

481.09

1.4111

1.4156

0.2273
458.44

0.2317

464.05
1.4010

0.2360

469.69

0.2403

475.33

0.2446

1. 3963

1.4057

1.4103

480.99

0.2281

$458.33 \quad 463.95$

1.3955

1.4002

0.2324

469.58

0.2367

475.23

0.2409

480.90

1.4049

1.4095

1.4140

0.2205

458.21

0.2247

463.84

1.3994

0.2289

463.48

1.4041

0.2331

475.13

0.2373

480.80

1.4087

1.4132

0.2172

1.3939

0.2214

0.2256

469.38

0.2297

475.03

0.2338

1.3987

1.4033

1.4079

480.71

0.2223

0.2264

474.94

0.2304

457.99

1.3932

463.63

1.3979

1.4026

I. 4072

480.61

0.2110

457.88

0.2150

463.52

1.3924

1.3972

0.2191

469.17

0.2231

474.84

0.2272

480.52

1.4018

1.4064

1.4110

$0.2080 \quad 0.2120$

457.77

1.3917

463.41
1.3964

0.2160

469.07

0.2200

1.4011

474.74
1.4057

0.2240

480.42

1.4102

$0.2009 \quad 0.2048$

457.49

1.3898

0.2087
468.81

1.3993
0.2125

474.49

1.4039
0.2164

480.19

1.4084
820

0.2609

486.94

1.4217

0.2568

486.85

1.4208

0.2528

486.76

1.4200

0.2489

486.67

1.4192

0.2451

486.58

1.4185

0.2415

486.48

1.4177

0.2379

486.39

1.4169

0.2345

486.30

1.4162

0.2312

486.21

1.4154

0.2279

486.12

1.4147

0.2202

485.89

1.4129
840

0.2654

492.62

1.4261

0.2612

492.53

I. 4252

0.2571

492.44

I. 4244

0.2532

492.36

1.4237

0.2493

492.27

1.4229

0.2456

492.18

1.4221

0.2420

492.09

1.4213

0.2385

492.01

1.4206

0.2352

491.92

1.4199

0.2319

491.83

1.4191

0.2240

491.61

1.4174
860

0.2698

498.31

1.4304

0.2655

498.23

1.4296

0.2614

498.15

1.4288

0.2574

498.06

1.4280

0.2535

497.98

1.4272

0.2498

497.89

1.4265

0.2461

497.81

1. 4257

0.2426

497.72

1.4250

0.2391

497.64

1.4242

0.2358

497.56

1.4235

0.2278

497.35

1.4217

880

0.2742

504.02

1.4347

0.2699

503.94

1.4339

0.2657

503.86

1.4331

0.2617

503.78

1.4323

\subsection{7}

$503 \cdot 70$

1.4315

0.2539

503.62

1.4308

0.2502

503.54

1.4300

0.2466

503.46

1.4293

0.2431

503.38

1.4285

0.2397 


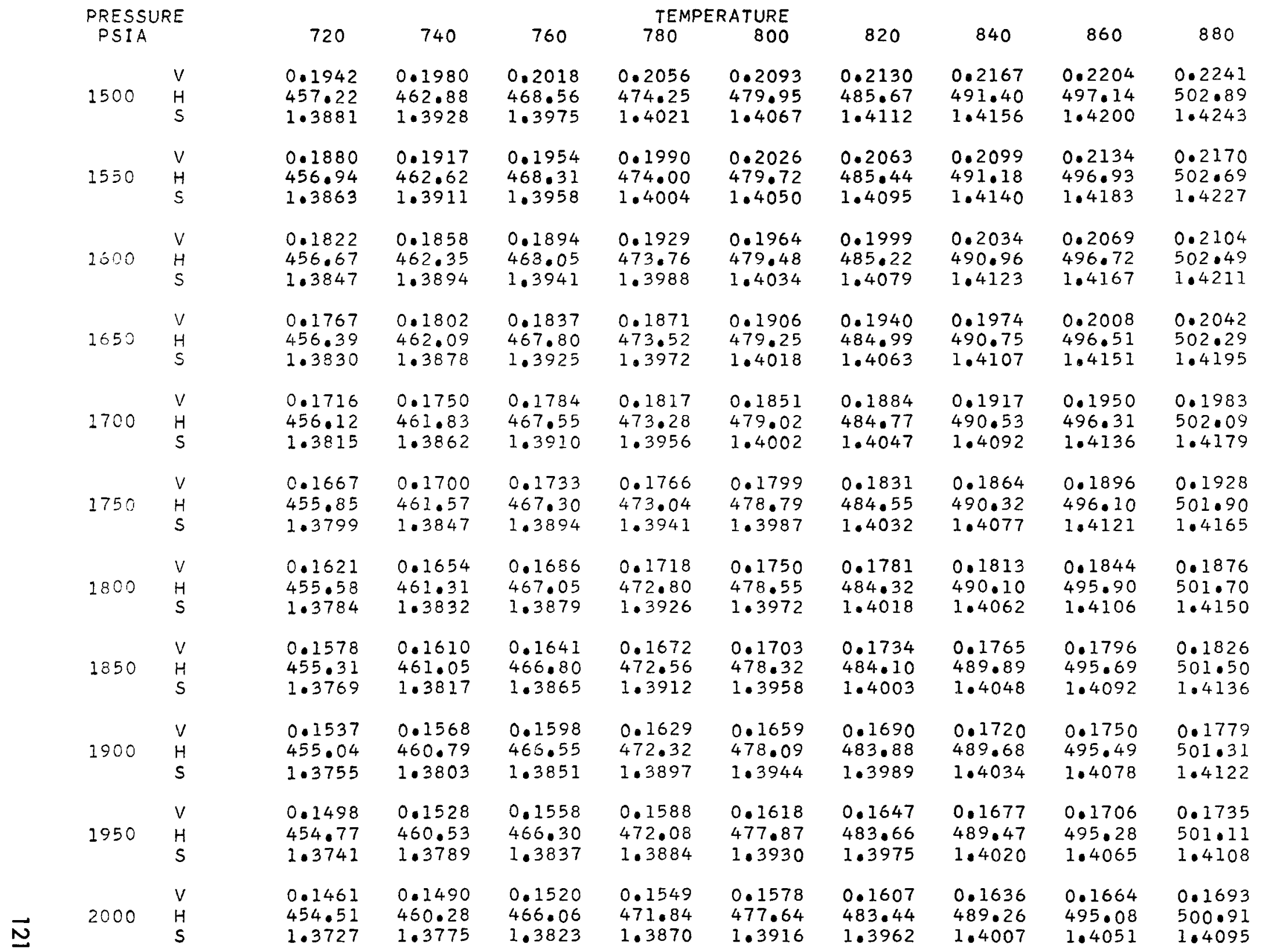




\begin{tabular}{|c|c|c|c|c|c|c|c|c|c|c|}
\hline 14.696 & $\begin{array}{l}V \\
H \\
S\end{array}$ & $\begin{array}{r}22.5544 \\
514.64 \\
1.6420\end{array}$ & $\begin{array}{r}22.8864 \\
520.18 \\
1.6460\end{array}$ & $\begin{array}{r}23.2185 \\
525.74 \\
1.6500\end{array}$ & $\begin{array}{r}23.5506 \\
531.32 \\
1.6540\end{array}$ & $\begin{array}{r}23.8826 \\
536.93 \\
1.6579\end{array}$ & $\begin{array}{r}24.2147 \\
542.55 \\
1.6618\end{array}$ & $\begin{array}{r}24.5467 \\
548.19 \\
1.6656\end{array}$ & $\begin{array}{r}24.8787 \\
553.85 \\
1.6694\end{array}$ & $\begin{array}{r}25.2107 \\
559.54 \\
1.6732\end{array}$ \\
\hline 16 & $\begin{array}{l}V \\
H \\
S\end{array}$ & $\begin{array}{r}20.7168 \\
514.63 \\
1.6382\end{array}$ & $\begin{array}{r}21.0219 \\
520.17 \\
1.6422\end{array}$ & $\begin{array}{r}21.3269 \\
525.74 \\
1.6462\end{array}$ & $\begin{array}{r}21.6320 \\
531.32 \\
1.6502\end{array}$ & $\begin{array}{r}21.9370 \\
536.92 \\
1.6541\end{array}$ & $\begin{array}{r}22.2420 \\
542.55 \\
1.6580\end{array}$ & $\begin{array}{r}22.5470 \\
548.19 \\
1.6618\end{array}$ & $\begin{array}{r}22.8520 \\
553.85 \\
1.6656\end{array}$ & $\begin{array}{r}23.1570 \\
559.53 \\
1.6693\end{array}$ \\
\hline 20 & $\begin{array}{l}V \\
H \\
S\end{array}$ & $\begin{array}{r}16.5751 \\
514.61 \\
1.6281\end{array}$ & $\begin{array}{r}16.8192 \\
520.16 \\
1.6322\end{array}$ & $\begin{array}{r}17.0634 \\
525.72 \\
1.6362\end{array}$ & $\begin{array}{r}17.3075 \\
531.30 \\
1.6401\end{array}$ & $\begin{array}{r}17.5516 \\
536.91 \\
1.6441\end{array}$ & $\begin{array}{r}17.7957 \\
542.53 \\
1.6479\end{array}$ & $\begin{array}{r}18.0397 \\
548.18 \\
1.6518\end{array}$ & $\begin{array}{r}18.2838 \\
553.84 \\
1.6556\end{array}$ & $\begin{array}{r}18.5278 \\
559.52 \\
1.6593\end{array}$ \\
\hline 24 & $\begin{array}{l}V \\
H \\
S\end{array}$ & $\begin{array}{r}13.8140 \\
514.60 \\
1.6199\end{array}$ & $\begin{array}{r}14.0175 \\
520.14 \\
1.6240\end{array}$ & $\begin{array}{r}14.2210 \\
525.70 \\
1.6280\end{array}$ & $\begin{array}{r}14.4245 \\
531.29 \\
1.6319\end{array}$ & $\begin{array}{r}14.6280 \\
536.89 \\
1.5359\end{array}$ & $\begin{array}{r}14.8314 \\
542.52 \\
1.6397\end{array}$ & $\begin{array}{r}15.0349 \\
548.16 \\
1.6436\end{array}$ & $\begin{array}{r}15.2383 \\
553.83 \\
1.6474\end{array}$ & $\begin{array}{r}15.4418 \\
559.51 \\
1.6511\end{array}$ \\
\hline 28 & $\begin{array}{l}V \\
H \\
S\end{array}$ & $\begin{array}{r}11.8417 \\
514.58 \\
1.6130\end{array}$ & $\begin{array}{r}12.0162 \\
520.12 \\
1.6171\end{array}$ & $\begin{array}{r}12.1907 \\
525.69 \\
1.5211\end{array}$ & $\begin{array}{r}12.3652 \\
531.27 \\
1.6250\end{array}$ & $\begin{array}{r}12.5397 \\
536.88 \\
1.6289\end{array}$ & $\begin{array}{r}12.7141 \\
542.50 \\
1.6328\end{array}$ & $\begin{array}{r}12.8886 \\
548.15 \\
1.6366\end{array}$ & $\begin{array}{r}13.0630 \\
553.81 \\
1.6404\end{array}$ & $\begin{array}{r}13.2374 \\
559.50 \\
1.6442\end{array}$ \\
\hline 32 & $\begin{array}{l}V \\
H \\
S\end{array}$ & $\begin{array}{r}10.3625 \\
514.56 \\
1.6070\end{array}$ & $\begin{array}{r}10.5153 \\
520.11 \\
1.6110\end{array}$ & $\begin{array}{r}10.5680 \\
525.67 \\
1.6150\end{array}$ & $\begin{array}{r}10.8207 \\
531.26 \\
1.6190\end{array}$ & $\begin{array}{r}10.9734 \\
536.87 \\
1.6229\end{array}$ & $\begin{array}{r}11.1261 \\
542.49 \\
1.6268\end{array}$ & $\begin{array}{r}11.2788 \\
548.14 \\
1.6306\end{array}$ & $\begin{array}{r}11.4315 \\
553.80 \\
1.6344\end{array}$ & $\begin{array}{r}11.5842 \\
559.48 \\
1.6382\end{array}$ \\
\hline 36 & $\begin{array}{l}V \\
H \\
S\end{array}$ & $\begin{array}{l}9.2120 \\
514.55 \\
1.6017\end{array}$ & $\begin{array}{l}9.3478 \\
520.09 \\
1.6057\end{array}$ & $\begin{array}{l}9.4837 \\
525.66 \\
1.6097\end{array}$ & $\begin{array}{l}9.6195 \\
531.24 \\
1.6137\end{array}$ & $\begin{array}{l}9.7553 \\
536.85 \\
1.6176\end{array}$ & $\begin{array}{l}9.8910 \\
542.48 \\
1.6215\end{array}$ & $\begin{array}{r}10.0268 \\
548.12 \\
1.6253\end{array}$ & $\begin{array}{r}10.1626 \\
553.79 \\
1.6291\end{array}$ & $\begin{array}{r}10.2983 \\
559.47 \\
1.6329\end{array}$ \\
\hline 40 & $\begin{array}{l}V \\
H \\
S\end{array}$ & $\begin{array}{l}8.2916 \\
514.53 \\
1.5970\end{array}$ & $\begin{array}{l}8.4139 \\
520.08 \\
1.6010\end{array}$ & $\begin{array}{l}8.5362 \\
525.64 \\
1.6050\end{array}$ & $\begin{array}{l}8.6585 \\
531.23 \\
1.609 n\end{array}$ & $\begin{array}{l}8.7807 \\
536.84 \\
1.6129\end{array}$ & $\begin{array}{l}8.9030 \\
542.46 \\
1.6168\end{array}$ & $\begin{array}{l}9.0252 \\
548.11 \\
1.6206\end{array}$ & $\begin{array}{l}9.1474 \\
553.78 \\
1.6244\end{array}$ & $\begin{array}{l}9.2696 \\
559.46 \\
1.6282\end{array}$ \\
\hline 44 & $\begin{array}{l}V \\
H \\
S\end{array}$ & $\begin{array}{l}7.5386 \\
514.51 \\
1.5927\end{array}$ & $\begin{array}{l}7.6498 \\
520.06 \\
1.5967\end{array}$ & $\begin{array}{l}7.7610 \\
525.63 \\
1.6007\end{array}$ & $\begin{array}{l}7.8722 \\
531.21 \\
1.6047\end{array}$ & $\begin{array}{l}7.9834 \\
536.82 \\
1.6086\end{array}$ & $\begin{array}{l}8.0945 \\
542.45 \\
1.6125\end{array}$ & $\begin{array}{l}8.2057 \\
548.10 \\
1.6163\end{array}$ & $\begin{array}{l}8.3168 \\
553.76 \\
1.6201\end{array}$ & $\begin{array}{l}8.4280 \\
559.45 \\
1.6239\end{array}$ \\
\hline 48 & $\begin{array}{l}V \\
H \\
S\end{array}$ & $\begin{array}{l}6.9110 \\
514.50 \\
1.5887\end{array}$ & $\begin{array}{l}7.0130 \\
520.04 \\
1.5928\end{array}$ & $\begin{array}{l}7.1150 \\
525.61 \\
1.5968\end{array}$ & $\begin{array}{l}7.2170 \\
531.20 \\
1.6008\end{array}$ & $\begin{array}{l}7.3189 \\
536.81 \\
1.6047\end{array}$ & $\begin{array}{l}7.4208 \\
542.44 \\
1.6086\end{array}$ & $\begin{array}{l}7.5228 \\
548.08 \\
1.6124\end{array}$ & $\begin{array}{l}7.6247 \\
553.75 \\
1.6162\end{array}$ & $\begin{array}{l}7.7266 \\
559.44 \\
1.6200\end{array}$ \\
\hline 52 & $\begin{array}{l}V \\
H \\
S\end{array}$ & $\begin{array}{l}6.3801 \\
514.48 \\
1.5851\end{array}$ & $\begin{array}{l}6.4742 \\
520.03 \\
1.5892\end{array}$ & $\begin{array}{l}6.5684 \\
525.60 \\
1.5932\end{array}$ & $\begin{array}{l}6.6625 \\
531.18 \\
1.5972\end{array}$ & $\begin{array}{l}6.7567 \\
536.79 \\
1.6011\end{array}$ & $\begin{array}{l}6.8508 \\
542.42 \\
1.6050\end{array}$ & $\begin{array}{l}6.9449 \\
548.07 \\
1.6088\end{array}$ & $\begin{array}{l}7.0390 \\
553.74 \\
1.6126\end{array}$ & $\begin{array}{l}7.1331 \\
559.43 \\
1.6164\end{array}$ \\
\hline
\end{tabular}




\begin{tabular}{|c|c|c|c|c|c|c|c|c|c|c|}
\hline \multirow{2}{*}{\multicolumn{2}{|c|}{$\begin{array}{l}\text { PRESSURE } \\
\text { PSIA }\end{array}$}} & \multicolumn{9}{|c|}{ TEMPERATURE } \\
\hline & & 900 & 920 & 940 & 960 & 980 & 1000 & 1020 & 1040 & 1060 \\
\hline 56 & $\begin{array}{l}V \\
H \\
S\end{array}$ & $\begin{array}{l}5.9249 \\
514.46 \\
1.5818\end{array}$ & $\begin{array}{l}6.0124 \\
520.01 \\
1.5858\end{array}$ & $\begin{array}{l}6.0999 \\
525.58 \\
1.5898\end{array}$ & $\begin{array}{l}6.1873 \\
531.17 \\
1.5938\end{array}$ & $\begin{array}{l}6.2748 \\
536.78 \\
1.5977\end{array}$ & $\begin{array}{l}6.3622 \\
542.41 \\
1.6016\end{array}$ & $\begin{array}{l}6.4496 \\
548.06 \\
1.6055\end{array}$ & $\begin{array}{l}6.5370 \\
553.73 \\
1.6093\end{array}$ & $\begin{array}{l}6.6244 \\
559.41 \\
1.6130\end{array}$ \\
\hline 60 & $\begin{array}{l}V \\
H \\
S\end{array}$ & $\begin{array}{l}5.5305 \\
514.45 \\
1.5787\end{array}$ & $\begin{array}{l}5.6121 \\
520.00 \\
1.5827\end{array}$ & $\begin{array}{l}5.6938 \\
525.56 \\
1.5867\end{array}$ & $\begin{array}{l}5.7754 \\
531.16 \\
1.5907\end{array}$ & $\begin{array}{l}5.8571 \\
536.77 \\
1.5946\end{array}$ & $\begin{array}{l}5.9387 \\
542.40 \\
1.5985\end{array}$ & $\begin{array}{l}6.0203 \\
548.05 \\
1.6023\end{array}$ & $\begin{array}{l}6.1019 \\
553.71 \\
1.6061\end{array}$ & $\begin{array}{l}6.1835 \\
559.40 \\
1.6099\end{array}$ \\
\hline 70 & $\begin{array}{l}V \\
H \\
S\end{array}$ & $\begin{array}{l}4.7415 \\
514.40 \\
1.5717\end{array}$ & $\begin{array}{l}4.8116 \\
519.95 \\
1.5758\end{array}$ & $\begin{array}{l}4.8817 \\
525.53 \\
1.5798\end{array}$ & $\begin{array}{l}4.9517 \\
531.12 \\
1.5837\end{array}$ & $\begin{array}{l}5.0218 \\
536.73 \\
1.5877\end{array}$ & $\begin{array}{l}5.0918 \\
542.36 \\
1.5916\end{array}$ & $\begin{array}{l}5.1618 \\
548.01 \\
1.5954\end{array}$ & $\begin{array}{l}5.2318 \\
553.68 \\
1.5992\end{array}$ & $\begin{array}{l}5.3018 \\
559.37 \\
1.6030\end{array}$ \\
\hline 80 & $\begin{array}{l}V \\
H \\
S\end{array}$ & $\begin{array}{l}4.1499 \\
514.36 \\
1.5657\end{array}$ & $\begin{array}{l}4.2112 \\
519.91 \\
1.5697\end{array}$ & $\begin{array}{l}4.2726 \\
525.49 \\
1.5738\end{array}$ & $\begin{array}{l}4.3339 \\
531.08 \\
1.5777\end{array}$ & $\begin{array}{l}4.3953 \\
536.70 \\
1.5816\end{array}$ & $\begin{array}{l}4.4566 \\
542.33 \\
1.5855\end{array}$ & $\begin{array}{l}4.5179 \\
547.98 \\
1.5894\end{array}$ & $\begin{array}{l}4.5792 \\
553.65 \\
1.5932\end{array}$ & $\begin{array}{l}4.6405 \\
559.34 \\
1.5969\end{array}$ \\
\hline 90 & $\begin{array}{l}V \\
H \\
S\end{array}$ & $\begin{array}{l}3.6897 \\
514.32 \\
1.5604\end{array}$ & $\begin{array}{l}3.7443 \\
519.87 \\
1.5644\end{array}$ & $\begin{array}{l}3.7989 \\
525.45 \\
1.5684\end{array}$ & $\begin{array}{l}3.8534 \\
531.04 \\
1.5724\end{array}$ & $\begin{array}{l}3.9080 \\
536.66 \\
1.5763\end{array}$ & $\begin{array}{l}3.9525 \\
542.30 \\
1.5302\end{array}$ & $\begin{array}{l}4.0171 \\
547.95 \\
1.5840\end{array}$ & $\begin{array}{l}4.0716 \\
553.62 \\
1.5879\end{array}$ & $\begin{array}{l}4.1261 \\
559.31 \\
1.5916\end{array}$ \\
\hline 100 & $\begin{array}{l}V \\
H \\
S\end{array}$ & $\begin{array}{l}3.3215 \\
514.22 \\
1.5556\end{array}$ & $\begin{array}{l}3.3707 \\
519.83 \\
1.5597\end{array}$ & $\begin{array}{l}3.4109 \\
525.41 \\
1.5637\end{array}$ & $\begin{array}{l}3.4690 \\
531.01 \\
1.5676\end{array}$ & $\begin{array}{l}3.5182 \\
536.62 \\
1.5716\end{array}$ & $\begin{array}{l}3.5673 \\
542.26 \\
1.5754\end{array}$ & $\begin{array}{l}3.6164 \\
547.92 \\
1.5793\end{array}$ & $\begin{array}{l}3.6655 \\
553.59 \\
1.5831\end{array}$ & $\begin{array}{l}3.7146 \\
559.28 \\
1.5869\end{array}$ \\
\hline 110 & $\begin{array}{l}V \\
H \\
S\end{array}$ & $\begin{array}{l}3.0203 \\
514.24 \\
1.5513\end{array}$ & $\begin{array}{l}3.0650 \\
519.79 \\
1.5553\end{array}$ & $\begin{array}{l}3.1098 \\
525.37 \\
1.5594\end{array}$ & $\begin{array}{l}3.1545 \\
530.97 \\
1.5633\end{array}$ & $\begin{array}{l}3.1992 \\
536.59 \\
1.5672\end{array}$ & $\begin{array}{l}3.2439 \\
542.23 \\
1.5711\end{array}$ & $\begin{array}{l}3.2886 \\
547.88 \\
1.5750\end{array}$ & $\begin{array}{l}3.3333 \\
553.56 \\
1.5788\end{array}$ & $\begin{array}{l}3.3780 \\
559.26 \\
1.5826\end{array}$ \\
\hline 120 & $\begin{array}{l}V \\
H \\
S\end{array}$ & $\begin{array}{l}2.7693 \\
514.20 \\
1.5473\end{array}$ & $\begin{array}{l}2.8103 \\
519.75 \\
1.5514\end{array}$ & $\begin{array}{l}2.8514 \\
525.33 \\
1.5554\end{array}$ & $\begin{array}{l}2.8924 \\
530.93 \\
1.5594\end{array}$ & $\begin{array}{l}2.9334 \\
536.55 \\
1.5633\end{array}$ & $\begin{array}{l}2.9744 \\
542.19 \\
1.5672\end{array}$ & $\begin{array}{l}3.0154 \\
547.85 \\
1.5710\end{array}$ & $\begin{array}{l}3.0564 \\
553.53 \\
1.5748\end{array}$ & $\begin{array}{l}3.0974 \\
559.23 \\
1.5786\end{array}$ \\
\hline 130 & $\begin{array}{l}V \\
H \\
S\end{array}$ & $\begin{array}{l}2.5569 \\
514.15 \\
1.5437\end{array}$ & $\begin{array}{l}2.5948 \\
519.71 \\
1.5478\end{array}$ & $\begin{array}{l}2.6327 \\
525.30 \\
1.5518\end{array}$ & $\begin{array}{l}2.6706 \\
530.90 \\
1.5557\end{array}$ & $\begin{array}{l}2.7085 \\
536.52 \\
1.5597\end{array}$ & $\begin{array}{l}2.7464 \\
542.16 \\
1.5636\end{array}$ & $\begin{array}{l}2.7843 \\
547.82 \\
1.5674\end{array}$ & $\begin{array}{l}2.8221 \\
553.50 \\
1.5712\end{array}$ & $\begin{array}{l}2.8600 \\
559.20 \\
1.5750\end{array}$ \\
\hline 140 & $\begin{array}{l}V \\
H \\
S\end{array}$ & $\begin{array}{l}2.3748 \\
514.11 \\
1.5403\end{array}$ & $\begin{array}{l}2.4100 \\
519.67 \\
1.5444\end{array}$ & $\begin{array}{l}2.4453 \\
525.26 \\
1.5484\end{array}$ & $\begin{array}{l}2.4805 \\
530.86 \\
1.5524\end{array}$ & $\begin{array}{l}2.5157 \\
536.48 \\
1.5563\end{array}$ & $\begin{array}{l}2.5510 \\
542.13 \\
1.5602\end{array}$ & $\begin{array}{l}2.5862 \\
547.79 \\
1.5641\end{array}$ & $\begin{array}{l}2.6213 \\
553.47 \\
1.5679\end{array}$ & $\begin{array}{l}2.6565 \\
559.17 \\
1.5716\end{array}$ \\
\hline 150 & $\begin{array}{l}V \\
H \\
S\end{array}$ & $\begin{array}{l}2.2170 \\
514.07 \\
1.5372\end{array}$ & $\begin{array}{l}2.2499 \\
519.63 \\
1.5413\end{array}$ & $\begin{array}{l}2.2829 \\
525.22 \\
1.5453\end{array}$ & $\begin{array}{l}2.3158 \\
530.82 \\
1.5493\end{array}$ & $\begin{array}{l}2.3487 \\
536.45 \\
1.5532\end{array}$ & $\begin{array}{l}2.3816 \\
542.09 \\
1.5571\end{array}$ & $\begin{array}{l}2.4144 \\
547.76 \\
1.5609\end{array}$ & $\begin{array}{l}2.4473 \\
553.44 \\
1.5647\end{array}$ & $\begin{array}{l}2.4802 \\
559.14 \\
1.5685\end{array}$ \\
\hline
\end{tabular}




\section{$\bar{N}$ PRESSURE \\ PSIA}

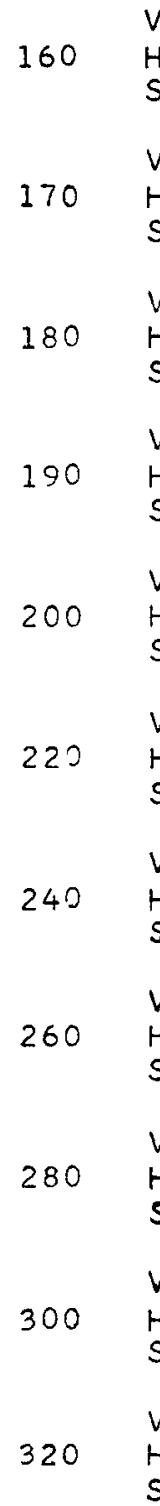

900

920

2.0789

514.03

1.5343

2.1098

519.59

1.5383

1.9571

513.99

1.5315

1.9862

519.55

1.5356

1.8488

513.95

1.5289

1.8763

519.51

1.5330

1.7519

513.90

1.7780

519.47

1.5305

1.6647

513.86

1.5241

1.6895

519.44

I. 5282

1.5141

513.78

1.5197

1.5367

519.36

1.5238

1.3886

513.70

1. 4093

519.28

1.5198

1.2824

513.61

1.5121

1.3016

519.20

1.5162

$1.1913 \quad 1.2092$

513.53

1.5687

1.1124

513.45

1.5055

1.0434

513.37

1.5025
519.12

1.5127

1.1291

519.04

1.5096

1.0590

518.96

1.5066
940

2. 1407

525.18

I. 5423

2.0153

525.14

1.5396

1.9039

525.10

1.5370

1.9041

525.07

1.5345

1.7143

525.03

1.5322

1.5593

524.95

1.5278

1.4301

524.88

1.5238

1.3207

524.80

1.5202

1.2270

524.72

I. 5168

1.1458

524.65

1.5136

1.0747

524.57

1.5106

\section{TEMPERATURE}

$960 \quad 980$

2.1716

530.79

2.2025

536.41

1.5503

2.0444

530.75

1.5436

2.0735

536.38

1.5475

1.9314

530.7

1.5410

1. 9588

536.34

1.5449

1.8302

530.68

1.5385

1.8562

536.31

1. 5424

1.7391

530.64

1.7639

536.27

1.5401

1.5819

530.57

1.6044

536.20

1.5357

1.5318

1.4715

1.4508

530.50

1. 5278

536.13
1.5318

1.3399

530.42

1.5242

1.3590

536.07

1.5281

1.2448

530.35

1. 5208

1.2626

536.00

1.5247

1. 1624

530.28

1.5176

I. 1791

535.93

1. 5215

1.0904

530.21

1. 5146

1. 1060

535.86

1. 5186
1000

2.2333

542.06

1. 5541

2.1026

542.03

1.5514

1.9863

541.99

I. 5488

1.8323

541.96

1.5463

1.7887

541.93

1. 5440

1.6270

541.86

1.5396

1.4922

541.79

1.5357

1.3782

541.73

1. 5320

1.2805

541.66

1.5286

1.1957

541.59

1.5255

1.1216

541.53

1.5225
1020

1040

1060

2.2642

547.72

2.2950

553.41

$1.5580 \quad 1.5618$

2.1316

547.69

1.5552

2.1607

553.38

1.5591

2.0138

547.66

1.5526

2.0412

553.35

1.9083

547.63

1.5502

1.9344

553.32

1.5540

1.8134

547.60

1.8382

553.29

1.5478

1.5517

1.6495

547.53

1.5435

553.22

1.5473

1.5129

547.47

1.5336

553.16

1.5434

1.3973

547.41

1.5359

1.4164

553.10

1.5397

1.2982

547.34

1.3160

553.04

1.5363

1.2124

547.28

1.5293

1.2290

552.98

1.5331

1.1372

547.22

1.1528

552.92

1.5302

2. 3259

559.11

1.5656

2.1897

559.08

1.5628

2.0687

559.05

1.5602

1.9604

559.02

1.8629

558.99

1.5554

1.6946

558.93

1. 5511 


\begin{tabular}{|c|c|c|c|c|c|c|c|c|c|c|}
\hline \multirow{2}{*}{\multicolumn{2}{|c|}{$\begin{array}{l}\text { PRESSURE } \\
\text { PSIA }\end{array}$}} & \multicolumn{9}{|c|}{ TEMPERATURE } \\
\hline & & 900 & 920 & 940 & 960 & 980 & 1000 & 1020 & 1040 & 1060 \\
\hline 340 & $\begin{array}{l}V \\
H \\
S\end{array}$ & $\begin{array}{l}0.9825 \\
513.28 \\
1.4997\end{array}$ & $\begin{array}{l}0.9972 \\
518.88 \\
1.5038\end{array}$ & $\begin{array}{l}1.0120 \\
524.50 \\
1.5078\end{array}$ & $\begin{array}{l}1.0267 \\
530.13 \\
1.5118\end{array}$ & $\begin{array}{l}1.0415 \\
535.79 \\
1.5158\end{array}$ & $\begin{array}{l}1.0562 \\
541.46 \\
1.5197\end{array}$ & $\begin{array}{l}1.0709 \\
547.15 \\
1.5236\end{array}$ & $\begin{array}{l}1.0856 \\
552.86 \\
1.5274\end{array}$ & $\begin{array}{l}1.1003 \\
558.59 \\
1.5312\end{array}$ \\
\hline 360 & $\begin{array}{l}V \\
H \\
S\end{array}$ & $\begin{array}{l}0.9283 \\
513.20 \\
1.4971\end{array}$ & $\begin{array}{l}0.9423 \\
518.80 \\
1.5012\end{array}$ & $\begin{array}{l}0.9562 \\
524.42 \\
1.5052\end{array}$ & $\begin{array}{l}0.9702 \\
530.06 \\
1.5092\end{array}$ & $\begin{array}{l}0.9841 \\
535.72 \\
1.5131\end{array}$ & $\begin{array}{l}0.9981 \\
541.40 \\
1.5171\end{array}$ & $\begin{array}{l}1.0119 \\
547.08 \\
1.5209\end{array}$ & $\begin{array}{l}1.0258 \\
552.79 \\
1.5248\end{array}$ & $\begin{array}{l}1.0397 \\
558.52 \\
1.5285\end{array}$ \\
\hline 380 & $\begin{array}{l}V \\
H \\
S\end{array}$ & $\begin{array}{l}0.8798 \\
513.12 \\
1.4946\end{array}$ & $\begin{array}{l}0.8931 \\
518.72 \\
1.4987\end{array}$ & $\begin{array}{l}0.9064 \\
52+.35 \\
1.5027\end{array}$ & $\begin{array}{l}0.9196 \\
529.99 \\
1.5067\end{array}$ & $\begin{array}{l}0.9327 \\
535.64 \\
1.5106\end{array}$ & $\begin{array}{l}0.9460 \\
541.32 \\
1.5146\end{array}$ & $\begin{array}{l}0.9592 \\
547.02 \\
1.5184\end{array}$ & $\begin{array}{l}0.9723 \\
552.73 \\
1.5223\end{array}$ & $\begin{array}{l}0.9855 \\
558.46 \\
1.5261\end{array}$ \\
\hline 400 & $\begin{array}{l}V \\
H \\
S\end{array}$ & $\begin{array}{l}0.8362 \\
513.04 \\
1.4922\end{array}$ & $\begin{array}{l}0.8489 \\
518.65 \\
1.4963\end{array}$ & $\begin{array}{l}0.3615 \\
524.27 \\
1.5003\end{array}$ & $\begin{array}{l}0.8740 \\
529.91 \\
1.5043\end{array}$ & $\begin{array}{l}0.8866 \\
535.57 \\
1.5083\end{array}$ & $\begin{array}{l}0.8991 \\
541.26 \\
1.5122\end{array}$ & $\begin{array}{l}0.9117 \\
546.96 \\
1.5161\end{array}$ & $\begin{array}{l}0.9242 \\
552.67 \\
1.5199\end{array}$ & $\begin{array}{l}0.9368 \\
558.41 \\
1.5237\end{array}$ \\
\hline 420 & $\begin{array}{l}V \\
H \\
S\end{array}$ & $\begin{array}{l}0.7968 \\
512.96 \\
1.4899\end{array}$ & $\begin{array}{l}0.8087 \\
518.56 \\
1.4940\end{array}$ & $\begin{array}{l}0.8208 \\
524.19 \\
1.4981\end{array}$ & $\begin{array}{l}0.8328 \\
529.84 \\
1.5021\end{array}$ & $\begin{array}{l}0.8448 \\
535.51 \\
1.5050\end{array}$ & $\begin{array}{l}0.8568 \\
541.19 \\
1.5099\end{array}$ & $\begin{array}{l}0.8687 \\
546.89 \\
1.5138\end{array}$ & $\begin{array}{l}0.8807 \\
552.61 \\
1.5176\end{array}$ & $\begin{array}{l}0.8927 \\
558.35 \\
1.5214\end{array}$ \\
\hline 440 & $\begin{array}{l}V \\
H \\
S\end{array}$ & $\begin{array}{l}0.7608 \\
512.87 \\
1.4877\end{array}$ & $\begin{array}{l}0.7723 \\
518.48 \\
1.4918\end{array}$ & $\begin{array}{l}0.7838 \\
524.12 \\
1.4959\end{array}$ & $\begin{array}{l}0.7953 \\
529.77 \\
1.4999\end{array}$ & $\begin{array}{l}0.8068 \\
535.44 \\
1.5039\end{array}$ & $\begin{array}{l}0.8182 \\
541.12 \\
1.5078\end{array}$ & $\begin{array}{l}0.8297 \\
546.83 \\
1.5117\end{array}$ & $\begin{array}{l}0.8411 \\
552.55 \\
1.5155\end{array}$ & $\begin{array}{l}0.8526 \\
558.29 \\
1.5193\end{array}$ \\
\hline 460 & $\begin{array}{l}\mathrm{V} \\
\mathrm{H} \\
\mathrm{S}\end{array}$ & $\begin{array}{l}0.7281 \\
512.79 \\
1.4857\end{array}$ & $\begin{array}{l}0.7391 \\
518.40 \\
1.4898\end{array}$ & $\begin{array}{l}0.7501 \\
524.04 \\
1.4938\end{array}$ & $\begin{array}{l}0.7611 \\
529.69 \\
1.4978\end{array}$ & $\begin{array}{l}0.7721 \\
535.37 \\
1.5018\end{array}$ & $\begin{array}{l}0.7831 \\
541.06 \\
1.5057\end{array}$ & $\begin{array}{l}0.7940 \\
546.77 \\
1.5096\end{array}$ & $\begin{array}{l}0.8050 \\
552.49 \\
1.5134\end{array}$ & $\begin{array}{l}0.8159 \\
558.23 \\
1.5172\end{array}$ \\
\hline 480 & $\begin{array}{l}V \\
H \\
S\end{array}$ & $\begin{array}{l}0.6980 \\
512.71 \\
1.4837\end{array}$ & $\begin{array}{l}0.7086 \\
518.33 \\
1.4878\end{array}$ & $\begin{array}{l}0.7192 \\
523.96 \\
1.4918\end{array}$ & $\begin{array}{l}0.7297 \\
529.62 \\
1.4958\end{array}$ & $\begin{array}{l}0.7403 \\
535.30 \\
1.4998\end{array}$ & $\begin{array}{l}0.7508 \\
540.99 \\
1.5037\end{array}$ & $\begin{array}{l}0.7614 \\
546.70 \\
1.5076\end{array}$ & $\begin{array}{l}0.7719 \\
552.43 \\
1.5115\end{array}$ & $\begin{array}{l}0.7824 \\
558.17 \\
1.5153\end{array}$ \\
\hline 500 & $\begin{array}{l}V \\
H \\
S\end{array}$ & $\begin{array}{l}0.6704 \\
512.62 \\
1.4818\end{array}$ & $\begin{array}{l}0.6806 \\
518.25 \\
1.4859\end{array}$ & $\begin{array}{l}0.6907 \\
523.89 \\
1.4899\end{array}$ & $\begin{array}{l}0.7009 \\
529.55 \\
1.4939\end{array}$ & $\begin{array}{l}0.7110 \\
535.23 \\
1.4979\end{array}$ & $\begin{array}{l}0.7212 \\
540.93 \\
1.5018\end{array}$ & $\begin{array}{l}0.7313 \\
546.64 \\
1.5057\end{array}$ & $\begin{array}{l}0.7414 \\
552.37 \\
1.5096\end{array}$ & $\begin{array}{l}0.7515 \\
558.12 \\
1.5134\end{array}$ \\
\hline 520 & $\begin{array}{l}V \\
H \\
S\end{array}$ & $\begin{array}{l}0.6449 \\
512.54 \\
1.4799\end{array}$ & $\begin{array}{l}0.6547 \\
518.17 \\
1.4840\end{array}$ & $\begin{array}{l}0.6645 \\
523.81 \\
1.4881\end{array}$ & $\begin{array}{l}0.6743 \\
529.48 \\
1.4921\end{array}$ & $\begin{array}{l}0.6840 \\
535.16 \\
1.4961\end{array}$ & $\begin{array}{l}0.6938 \\
540.86 \\
1.5000\end{array}$ & $\begin{array}{l}0.7035 \\
546.58 \\
1.5039\end{array}$ & $\begin{array}{l}0.7133 \\
552.31 \\
1.5077\end{array}$ & $\begin{array}{l}0.7230 \\
558.06 \\
1.5115\end{array}$ \\
\hline 540 & $\begin{array}{l}V \\
H \\
S\end{array}$ & $\begin{array}{l}0.6213 \\
512.46 \\
1.4782\end{array}$ & $\begin{array}{l}0.6307 \\
518.09 \\
1.4823\end{array}$ & $\begin{array}{l}0.6402 \\
523.74 \\
1.4863\end{array}$ & $\begin{array}{l}0.6496 \\
529.41 \\
1.4903\end{array}$ & $\begin{array}{l}0.6590 \\
535.09 \\
1.4943\end{array}$ & $\begin{array}{l}0.6684 \\
540.79 \\
1.4982\end{array}$ & $\begin{array}{l}0.6778 \\
546.51 \\
1.5021\end{array}$ & $\begin{array}{l}0.6872 \\
552.25 \\
1.5060\end{array}$ & $\begin{array}{l}0.6966 \\
558.00 \\
1.5098\end{array}$ \\
\hline
\end{tabular}


0.5789

\subsection{7}

0.5966

1.4886

1.4926

1.4965

1. 5004

552.19

557.9

580

512.30

517.93

523.59

0.6054

0.6142

0.6229

0.6317

529.26

534.95

540.66

546.39

0.6405

552.13

1.4910

1.4949

1. 4988

1.5026

0.6492

557.89

$0.5599 \quad 0.5684$

512.22

1.4732

517.86

0.5769

0.5855

523.52

529.19

0.5940

534.88

0.6025

540.60

0.6110

0.6110
546.32

1.4972

0.6195

552.07

1.5011

0.6279
557.83

1.5049

$\begin{array}{ll}0.5420 & 0.5503 \\ 512.14 & 517.78\end{array}$

0.5586

523.44

0.5668

$529 \cdot 12$

0.5751

534.82

0.5333

1.4716

1.4758

1.4798

1.4838

1. 4878

540.53

0.5916

546.26

0.5998

552.01

0.6030 557.77

1.5033

512.05

0.5333

0.5414

0.5494

0.5574

0.5654

17.70

3.37

529.05

534.75

540.46

0.5734

1.4995

0.5893

$551.95 \quad 557.71$

1.4823

1.4863

1.4303

546.20

1.5018

$0.5096 \quad 0.5174$

0.5252

0.5330

0.5408

0.5485

0.5563

528.98

534.68

540.40

1.4888

546.14

1.4927

0.5640

0.5640

0.5713

1.4769

0.5176

1.4849

0.5327

0.5100

523.22

528.91

0.5251
534.61

I. 4835

540.33

0.5402

546.07

1.4913

1.4966

557.66

0.5477

0.5477
551.83

1.4952

0.5552

557.60

1.4990

0.4809

511.81

0.4883

517.47

0.4956

$523 \cdot 14$

0.5030

0.5103

0.5177

528.83

534.54

540.27

0.5250

546.01

0.5323

551.77

0.5396 557.54

1.4976

$0.4677 \quad 0.4749$

$511.73 \quad 517.39$

0.4821

0.4892

0.4964

0.5036

1. 4938

0.5249

$528.76 \quad 534.47$

540.20

0.510

0.5178

551.71

557.48

1.4727

1. 4768

1. 4808

1.4847

1.4886

1.4925

1.4963

$\begin{array}{lll}0.4552 & 0.4623 & 0.4693\end{array}$

0.4762

0.4832

0.4902

0.4971

0.5041

551.65

0.5110

528.69
1.4755

534.41
1.4795

540.14

545.88

1.4912

557.43

1.4714

0.4639

$0.4434 \quad 0.4503$

$0.4434 \quad 0.4503 \quad 0.4571$

511.57

517.24
1.4661

522.92

1.4702

0.4707

0.4775

1.4873

0.4911

0.4843

528.62

534.34

540.07

I. 4822

1.4861

551.59

1.4899

0.4978 557.37 1.4938 


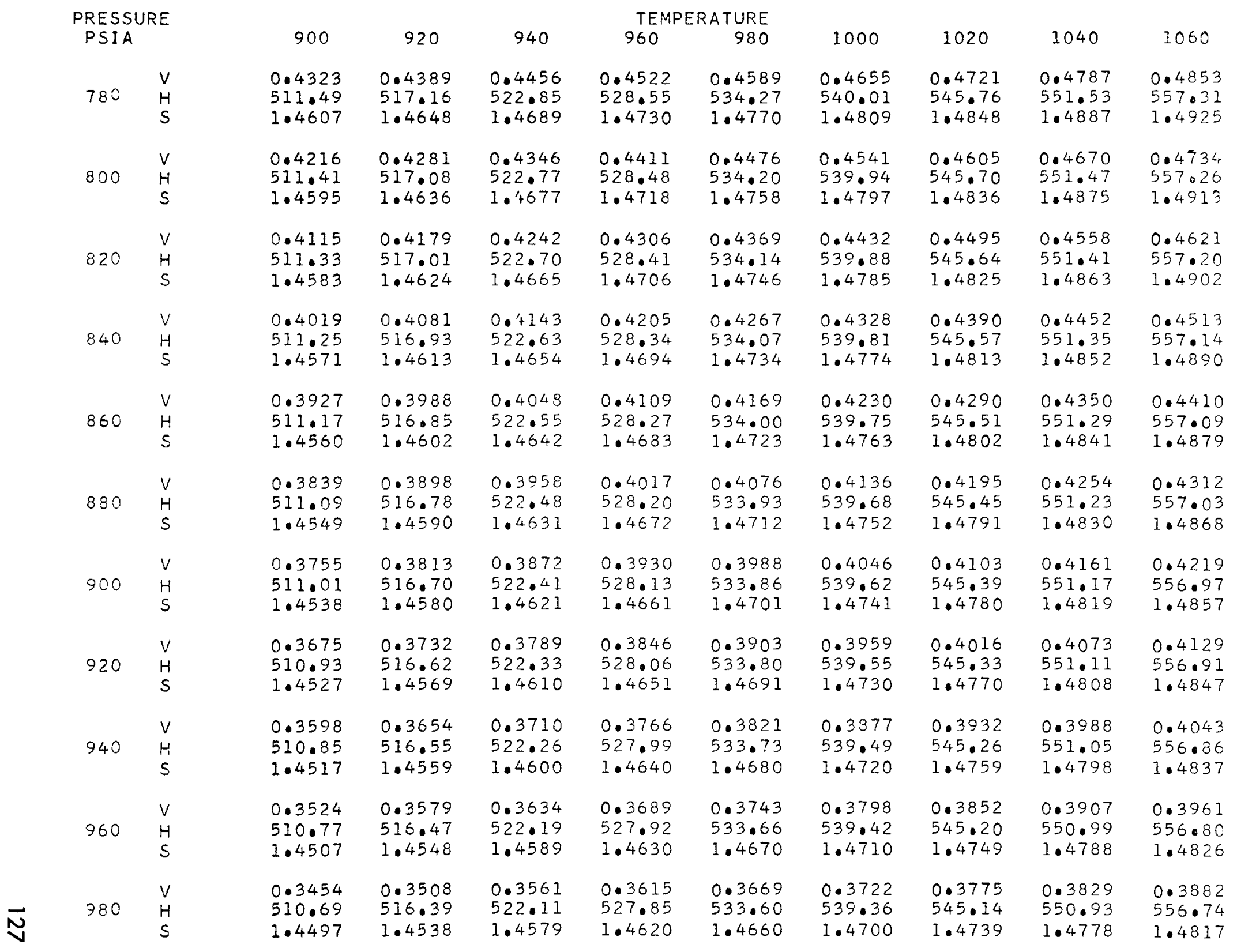




\section{$\bar{N}$ PRESSURE}

$$
\text { PSIA }
$$

1000

1020

1040

1060

1080

1100

2220

1140

1160

1180

2200
0.3386 510.61

1.4487

0.3321

510.53

1.4477

0.3258

510.46

1.4468

0.3198

510.38

1.4458

0.3140

510.30

1.4449

0.3084

510.22

1.4440

0.3030

510.14

1.4431

0.2978

1.4423

0.2928

509.98

1.4414

0.2879

509.90

1.4406

0.2832

509.83

1.4397
0.3439

516.32

1.4529

0.3373

516.24

1.4519

0.3309

516.17

1.4509

0.3248

516.09

1.4500

0.3189

516.02

1.4491

0.3132

515.94

1.4482

0.3078

515.86

1.4473

0.3025

515.79

1.4465

0.2974

515.71

1.4456

0.2924

515.64

1.4448

0.2877

515.56

1.4439
940

0.3492

522.04

1.4570

0.3425

521.97

1.4560

0.3360

521.89

1.4551

0.3298

521.82

1.4541

0.3238
521.75

1. +532

0.318

521.68

1.4523

0.3125

521.60

1.4514

0.3072

521.53

1.4506

\subsection{0}

521.46

1.4497

0.2970

521.39

1.4489

0.2921

521.31

1.4481

\section{TEMPERATURE}

$960 \quad 980$

0.3544
527.78

0.3476

527.71

1.4601

0.3411

527.64
1.4591

0.3348

527.57

1. 4582

0.3287

I. 457

0.3229

527.43

1.4564

0.3173

527.36
1.4555

0.3118

527.29

1.4547

\subsection{6}

527.22

1.4538

0.3015

527.15

1. 4530

0.2966

527.08

I. 4521
0.3597
533.53

0.3528

533.46

1.4641

0.3462

533.39

1.4632

0.3398

533.33

1.4622

0.3336

533.26

1.4613

0.3277

533.19

1.4604

0.3220

533.13

1.4596

0.3165

533.06

1.4587

0.3111

532.99

1.4578

0.3060

532.93

1.4570

0.3010

532.86

1.4562
1000

0.3649

539.30

1.4690

0.3579

539.23

1.4681

0.3512

539.17

1.4671

0.3447

539.10

1.4662

0.3385

539.04

1.4653

0.3325

538.97

1.4644

0.3267

538.91

1.4635

0.3211

538.85

1.4527

0.3157

538.78

1.4618

0.3105

538.72

1.4610

0.3054

538.65

1.4602
1020

0.3702

545.08

1.4730

0.3631

545.02

1.4720

0.3563

544.95

1.4711

0.3497

544.89

1.4701

0.3434

544.83

1.4692

0.3373

544.77

1. 4684

0.3314

544.71

1.4675

544.65

1.4666

0.3203

544.59

1.4658

0.3150

544.52

1.4649

0.3099

544.46

1.4641

1040

1060

0.3754

550.88

1.4768

0.3806

556.69

1. 4807

0.3682

550.82

1.4759

0.3733

556.63

1.4797

$0.3613 \quad 0.3663$

$550.76 \quad 556.57$

$1.4750 \quad 1.4788$

$0.3546 \quad 0.3596$

$550.70 \quad 556.52$

$1.4740 \quad 1.4779$

$0.3482 \quad 0.3531$

$550.64 \quad 556.46$

$1.4731 \quad 1.4770$

$0.3421 \quad 0.3468$

$550.58 \quad 556.41$

$1.4723 \quad 1.4761$

$\begin{array}{lll}0.3361 & 0.3408\end{array}$

$550.52 \quad 556.35$

$1.4714 \quad 1.4752$

$0.3304 \quad 0.3350$

$550.46 \quad 556.29$ 


\begin{tabular}{|c|c|c|c|c|c|c|c|c|c|c|}
\hline \multirow{2}{*}{\multicolumn{2}{|c|}{$\begin{array}{l}\text { PRESSURE } \\
\text { PSIA }\end{array}$}} & \multicolumn{9}{|c|}{$\begin{array}{l}\text { TEMPERATURE } \\
960 \quad 980\end{array}$} \\
\hline & & 900 & 920 & 940 & 960 & 980 & 1000 & 1020 & 1040 & 1060 \\
\hline 1220 & $\begin{array}{l}V \\
H \\
S\end{array}$ & $\begin{array}{l}0.2787 \\
509.75 \\
1.4389\end{array}$ & $\begin{array}{l}0.2831 \\
515.49 \\
1.4431\end{array}$ & $\begin{array}{l}0.2875 \\
521.24 \\
1.4472\end{array}$ & $\begin{array}{l}0.2918 \\
527.01 \\
1.4513\end{array}$ & $\begin{array}{l}0.2962 \\
532.79 \\
1.4554\end{array}$ & $\begin{array}{l}0.3006 \\
538.59 \\
1.4594\end{array}$ & $\begin{array}{l}0.3049 \\
544.40 \\
1.4633\end{array}$ & $\begin{array}{l}0.3093 \\
550.23 \\
1.4672\end{array}$ & $\begin{array}{l}0.3136 \\
556.07 \\
1.4711\end{array}$ \\
\hline 1240 & $\begin{array}{l}V \\
H \\
S\end{array}$ & $\begin{array}{l}0.2743 \\
509.67 \\
1.4381\end{array}$ & $\begin{array}{l}0.2786 \\
515.41 \\
1.4423\end{array}$ & $\begin{array}{l}0.2829 \\
521.17 \\
1.4464\end{array}$ & $\begin{array}{l}0.2872 \\
526.94 \\
1.4505\end{array}$ & $\begin{array}{l}0.2915 \\
532.73 \\
1.4546\end{array}$ & $\begin{array}{l}0.2958 \\
538.53 \\
1.4586\end{array}$ & $\begin{array}{l}0.3001 \\
544.34 \\
1.4625\end{array}$ & $\begin{array}{l}0.3044 \\
550.17 \\
1.4664\end{array}$ & $\begin{array}{l}0.3087 \\
556.01 \\
1.4703\end{array}$ \\
\hline 1260 & $\begin{array}{l}V \\
H \\
S\end{array}$ & $\begin{array}{l}0.2700 \\
509.59 \\
1.4373\end{array}$ & $\begin{array}{l}0.2743 \\
515.34 \\
1.4415\end{array}$ & $\begin{array}{l}0.2785 \\
521.10 \\
1.4457\end{array}$ & $\begin{array}{l}0.2828 \\
526.87 \\
1.4498\end{array}$ & $\begin{array}{l}0.2870 \\
532.66 \\
1.4538\end{array}$ & $\begin{array}{l}0.2913 \\
538.46 \\
1.4578\end{array}$ & $\begin{array}{l}0.2955 \\
544.28 \\
1.46 .17\end{array}$ & $\begin{array}{l}0.2997 \\
550.11 \\
1.4657\end{array}$ & $\begin{array}{l}0.3039 \\
555.96 \\
1.4695\end{array}$ \\
\hline 1280 & $\begin{array}{l}V \\
H \\
S\end{array}$ & $\begin{array}{l}0.2659 \\
509.51 \\
1.4365\end{array}$ & $\begin{array}{l}0.2701 \\
515.26 \\
1.4407\end{array}$ & $\begin{array}{l}0.2743 \\
521.03 \\
1.4449\end{array}$ & $\begin{array}{l}0.2785 \\
526.80 \\
1.4490\end{array}$ & $\begin{array}{l}0.2827 \\
532.59 \\
1.4530\end{array}$ & $\begin{array}{l}0.2868 \\
538.40 \\
1.4570\end{array}$ & $\begin{array}{l}0.2910 \\
544.22 \\
1.4610\end{array}$ & $\begin{array}{l}0.2952 \\
550.05 \\
1.4649\end{array}$ & $\begin{array}{l}0.2993 \\
555.90 \\
1.4688\end{array}$ \\
\hline 1300 & $\begin{array}{l}V \\
H \\
S\end{array}$ & $\begin{array}{l}0.2619 \\
509.44 \\
1.4358\end{array}$ & $\begin{array}{l}0.2660 \\
515.19 \\
1.4400\end{array}$ & $\begin{array}{l}0.2702 \\
520.95 \\
1.4441\end{array}$ & $\begin{array}{l}0.2743 \\
526.73 \\
1.4482\end{array}$ & $\begin{array}{l}0.2784 \\
532.53 \\
1.4523\end{array}$ & $\begin{array}{l}0.2825 \\
538.34 \\
1.4563\end{array}$ & $\begin{array}{l}0.2866 \\
544.16 \\
1.4602\end{array}$ & $\begin{array}{l}0.2907 \\
549.99 \\
1.4641\end{array}$ & $\begin{array}{l}0.2948 \\
555.84 \\
1.4680\end{array}$ \\
\hline 1320 & $\begin{array}{l}V \\
H \\
S\end{array}$ & $\begin{array}{l}0.2580 \\
509.36 \\
1.4350\end{array}$ & $\begin{array}{l}0.2621 \\
515.11 \\
1.4392\end{array}$ & $\begin{array}{l}0.2662 \\
520.88 \\
1.4434\end{array}$ & $\begin{array}{l}0.2703 \\
526.66 \\
1.4475\end{array}$ & $\begin{array}{l}0.2743 \\
532.46 \\
1.4515\end{array}$ & $\begin{array}{l}0.2784 \\
538.27 \\
1.4555\end{array}$ & $\begin{array}{l}0.2824 \\
544.10 \\
1.4595\end{array}$ & $\begin{array}{l}0.2865 \\
549.94 \\
1.4634\end{array}$ & $\begin{array}{l}0.2905 \\
555.79 \\
1.4673\end{array}$ \\
\hline 1340 & $\begin{array}{l}V \\
H \\
S\end{array}$ & $\begin{array}{l}0.2542 \\
509.28 \\
1.4343\end{array}$ & $\begin{array}{l}0.2583 \\
515.04 \\
1.4385\end{array}$ & $\begin{array}{l}0.2623 \\
520.81 \\
1.4426\end{array}$ & $\begin{array}{l}0.2663 \\
526.60 \\
1.4467\end{array}$ & $\begin{array}{l}0.2703 \\
532.40 \\
1.4508\end{array}$ & $\begin{array}{l}0.2743 \\
538.21 \\
1.4548\end{array}$ & $\begin{array}{l}0.2783 \\
544.04 \\
1.4587\end{array}$ & $\begin{array}{l}0.2823 \\
549.88 \\
1.4626\end{array}$ & $\begin{array}{l}0.2863 \\
555.73 \\
1.4665\end{array}$ \\
\hline 1360 & $\begin{array}{l}\mathrm{V} \\
\mathrm{H} \\
\mathrm{S}\end{array}$ & $\begin{array}{l}0.2506 \\
509.20 \\
1.4335\end{array}$ & $\begin{array}{l}0.2546 \\
514.96 \\
1.4377\end{array}$ & $\begin{array}{l}0.2586 \\
520.74 \\
1.4419\end{array}$ & $\begin{array}{l}0.2625 \\
526.53 \\
1.4460\end{array}$ & $\begin{array}{l}0.2665 \\
532.33 \\
1.4500\end{array}$ & $\begin{array}{l}0.2704 \\
538.15 \\
1.4540\end{array}$ & $\begin{array}{l}0.2744 \\
543.98 \\
1.4580\end{array}$ & $\begin{array}{l}0.2783 \\
549.82 \\
1.4619\end{array}$ & $\begin{array}{l}0.2822 \\
555.68 \\
1.4658\end{array}$ \\
\hline 1380 & $\begin{array}{l}V \\
H \\
S\end{array}$ & $\begin{array}{l}0.2470 \\
509.12 \\
1.4328\end{array}$ & $\begin{array}{l}0.2510 \\
514.89 \\
1.4370\end{array}$ & $\begin{array}{l}0.2549 \\
520.67 \\
1.4411\end{array}$ & $\begin{array}{l}0.2588 \\
526.46 \\
1.4453\end{array}$ & $\begin{array}{l}0.2627 \\
532.26 \\
1.4493\end{array}$ & $\begin{array}{l}0.2666 \\
538.08 \\
1.4533\end{array}$ & $\begin{array}{l}0.2705 \\
543.91 \\
1.4573\end{array}$ & $\begin{array}{l}0.2744 \\
549.76 \\
1.4612\end{array}$ & $\begin{array}{l}0.2782 \\
555.62 \\
1.4651\end{array}$ \\
\hline 1400 & $\begin{array}{l}V \\
H \\
S\end{array}$ & $\begin{array}{l}0.2436 \\
509.05 \\
1.4321\end{array}$ & $\begin{array}{l}0.2475 \\
514.81 \\
1.4363\end{array}$ & $\begin{array}{l}0.2513 \\
520.59 \\
1.4404\end{array}$ & $\begin{array}{l}0.2552 \\
526.39 \\
1.4445\end{array}$ & $\begin{array}{l}0.2591 \\
532.20 \\
1.4486\end{array}$ & $\begin{array}{l}0.2529 \\
538.02 \\
1.4526\end{array}$ & $\begin{array}{l}0.2667 \\
543.85 \\
1.4566\end{array}$ & $\begin{array}{l}0.2706 \\
549.70 \\
1.4605\end{array}$ & $\begin{array}{l}0.2744 \\
555.56 \\
1.4644\end{array}$ \\
\hline 1450 & $\begin{array}{l}V \\
H \\
S\end{array}$ & $\begin{array}{l}0.2354 \\
508.85 \\
1.4303\end{array}$ & $\begin{array}{l}0.2392 \\
514.63 \\
1.4345\end{array}$ & $\begin{array}{l}0.2429 \\
520.42 \\
1.4387\end{array}$ & $\begin{array}{l}0.2466 \\
526.22 \\
1.4428\end{array}$ & $\begin{array}{l}0.2504 \\
532.03 \\
1.4469\end{array}$ & $\begin{array}{l}0.2541 \\
537.86 \\
1.4509\end{array}$ & $\begin{array}{l}0.2578 \\
543.70 \\
1.4548\end{array}$ & $\begin{array}{l}0.2615 \\
549.56 \\
1.4588\end{array}$ & $\begin{array}{l}0.2652 \\
555.43 \\
1.4627\end{array}$ \\
\hline
\end{tabular}




$\begin{array}{ll}V & 0.2277\end{array}$

0.2314

$1500 \mathrm{H} \quad 508.66$

514.44

0.2350

960

980

1.4286

1.4328

520.24

0.2386

0.2423

526.05

531.87

1.4370

1.4411

1.4452

$0.2206 \quad 0.2241$

1550

508.47

0.2276

1.4312

520.06
1.4353

0.2312

0.2347

525.88

531.70

1.4395

1.4435

0.2138

508.28

0.2173

514.07

0.2207

1.4253

1.4296

519.88
1.4337

0.2241

525.71

0.2276

0.2075

508.09

0.2109

513.89

0.2142

1.4379

531.54

0.2175

525.54

0.2209

531.38

1.4322

1. 4363

1.4404

0.2016

507.89

0.2048

0.2081

0.2113

0.2146

519.53

525.37

531.21

1.4389

1.4265

1.4307

1.4348

0.2086

0.1960

0.1991

$507 \cdot 70$

513.52

0.2023

517.35

1.4292

0.2055

525.20

531.05

1.4374

0.1907

0.1938

513.34

0.1969

519.18

0.1999

525.03

0.2030

530.89

1.4360

0.1947

0.1977

0.1917

519.00

524.86

530.73

507.32

513.16

1.4264

1.4305

1.4346

0.1897

0.1927

0.1868

0.1809

0.1839

518.83

1.4165

1.4208

1.4250

524.69

530.57

1.4332

0.1822

518.65

0.1850

524.52

0.1879

530.40

1.4319

1.4194

1.4236

0.1833

0.1777
518.48

0.1805

524.36

530.24

1.4306

$\begin{array}{ll}512.61 & 518.48 \\ 1.4181 & 1.4223\end{array}$

1.4265

1000

\section{0}

0.2459

537.70

1.4492

0.2495

543.55

1.4532

0.2382

537.55

1.4476

0.2417

543.40

1.4515

$0.2310 \quad 0.2343$

537.39

1.4460

543.25

1.4500

0.2242

537.23

0.2275

543.10

1.4444

1.4484

0.2178

537.08

1.4429

0.2210

542210

1.4469

0.2118

536.92

0.2149

542.80

1.4455

0.2061

536.76

0.2091

542.65

$1.4400 \quad 1.4440$

0.2037

542.50

0.2007

536.61

1.4427

0.1956

536.45

0.1985

542.35

1.4413

0.1907

536.30

0.1936

542.20

1.4400

0.1861

536.14

0.1889

542.05

1.4387

\section{0}

1060

0.2531

549.41

1.4571

0.2566

555.29

$0.2451 \quad 0.2486$

$549.27 \quad 555.15$

1.45551 .4594

$0.2377 \quad 0.2411$

$549.12 \quad 555.01$

$1.4539 \quad 1.4578$

$0.2308 \quad 0.2340$

$548.98 \quad 554.87$

$1.4524 \quad 1.4563$

$0.2242 \quad 0.2274$

548.83

$1.4509 \quad 1.4548$

$0.2180 \quad 0.2211$

$548.69 \quad 554.59$

$1.4494 \quad 1.4533$

$0.2122 \quad 0.2152$

$548.55 \quad 554.46$

$1.4480 \quad 1.4519$

$0.2066 \quad 0.2096$

$548.40 \quad 554.32$

$1.4466 \quad 1.4505$

$0.2014 \quad 0.2043$

$548.26 \quad 554.18$

$1.4453 \quad 1.4492$

0.19640 .1992

$548.12 \quad 554.04$

$1.4440 \quad 1.4479$

$0.1917 \quad 0.1944$

$547.97 \quad 553.91$

$1.4427 \quad 1.4466$ 


\begin{tabular}{|c|c|c|c|c|c|c|c|c|c|c|}
\hline \multicolumn{2}{|c|}{$\begin{array}{l}\text { PRESSURE } \\
\text { PSIA }\end{array}$} & \multicolumn{9}{|c|}{ TEMPERATURE } \\
\hline 14.696 & $\begin{array}{l}V \\
H \\
S\end{array}$ & $\begin{array}{r}25.5427 \\
565.23 \\
1.6769\end{array}$ & $\begin{array}{r}25.8747 \\
570.95 \\
1.6806\end{array}$ & $\begin{array}{r}26.2067 \\
576.69 \\
1.6842\end{array}$ & $\begin{array}{r}26.5386 \\
582.44 \\
1.6878\end{array}$ & $\begin{array}{r}26.8706 \\
588.21 \\
1.6914\end{array}$ & $\begin{array}{r}27.2025 \\
593.99 \\
1.6950\end{array}$ & $\begin{array}{r}27.5345 \\
599.79 \\
1.6985\end{array}$ & $\begin{array}{r}27.8664 \\
605.61 \\
1.7020\end{array}$ & $\begin{array}{r}28.1983 \\
611.45 \\
1.7054\end{array}$ \\
\hline 16 & $\begin{array}{l}V \\
H \\
S\end{array}$ & $\begin{array}{r}23.4619 \\
565.23 \\
1.6731\end{array}$ & $\begin{array}{r}23.7669 \\
570.95 \\
1.6768\end{array}$ & $\begin{array}{r}24.0718 \\
575.68 \\
1.5804\end{array}$ & $\begin{array}{r}24.3768 \\
582.44 \\
1.6840\end{array}$ & $\begin{array}{r}24.6817 \\
588.20 \\
1.6876\end{array}$ & $\begin{array}{r}24.9866 \\
593.99 \\
1.6912\end{array}$ & $\begin{array}{r}25.2915 \\
599.79 \\
1.6947\end{array}$ & $\begin{array}{r}25.5964 \\
605.61 \\
1.6981\end{array}$ & $\begin{array}{r}25.90: 3 \\
611.44 \\
1.7016\end{array}$ \\
\hline 20 & $\begin{array}{l}V \\
H \\
S\end{array}$ & $\begin{array}{r}18.7719 \\
565.22 \\
1.6630\end{array}$ & $\begin{array}{r}19.0159 \\
570.94 \\
1.6667\end{array}$ & $\begin{array}{r}19.2599 \\
576.67 \\
1.6704\end{array}$ & $\begin{array}{r}19.5040 \\
582.43 \\
1.6740\end{array}$ & $\begin{array}{r}19.7480 \\
588.20 \\
1.6776\end{array}$ & $\begin{array}{r}19.9920 \\
593.98 \\
1.6811\end{array}$ & $\begin{array}{r}20.2360 \\
599.78 \\
1.6846\end{array}$ & $\begin{array}{r}20.4799 \\
605.60 \\
1.6881\end{array}$ & $\begin{array}{r}20.7239 \\
611.44 \\
1.6916\end{array}$ \\
\hline 24 & $\begin{array}{l}V \\
H \\
S\end{array}$ & $\begin{array}{r}15.6452 \\
565.21 \\
1.6549\end{array}$ & $\begin{array}{r}15.8486 \\
570.93 \\
1.6585\end{array}$ & $\begin{array}{r}16.0520 \\
576.66 \\
1.6622\end{array}$ & $\begin{array}{r}16.2554 \\
582.42 \\
1.6558\end{array}$ & $\begin{array}{r}16.4588 \\
588.19 \\
1.6694\end{array}$ & $\begin{array}{r}16.6522 \\
593.97 \\
1.6729\end{array}$ & $\begin{array}{r}16.8656 \\
599.78 \\
1.5765\end{array}$ & $\begin{array}{r}17.0690 \\
605.59 \\
1.6799\end{array}$ & $\begin{array}{r}17.2723 \\
611.43 \\
1.6834\end{array}$ \\
\hline 28 & $\begin{array}{l}V \\
H \\
S\end{array}$ & $\begin{array}{r}13.4119 \\
565.20 \\
1.6479\end{array}$ & $\begin{array}{r}13.5863 \\
570.92 \\
1.6516\end{array}$ & $\begin{array}{r}13.7607 \\
576.65 \\
1.3553\end{array}$ & $\begin{array}{r}13.9351 \\
582.41 \\
1.6589\end{array}$ & $\begin{array}{r}14.1094 \\
588.18 \\
1.6625\end{array}$ & $\begin{array}{r}14.2838 \\
593.96 \\
1.6660\end{array}$ & $\begin{array}{r}14.4582 \\
599.77 \\
1.6695\end{array}$ & $\begin{array}{r}14.6325 \\
605.59 \\
1.6730\end{array}$ & $\begin{array}{r}14.8069 \\
611.42 \\
1.6765\end{array}$ \\
\hline 32 & $\begin{array}{l}V \\
H \\
S\end{array}$ & $\begin{array}{r}11.7368 \\
565.19 \\
1.6419\end{array}$ & $\begin{array}{r}11.8895 \\
570.91 \\
1.6456\end{array}$ & $\begin{array}{r}12.042 .1 \\
576.64 \\
1.0493\end{array}$ & $\begin{array}{r}12.1948 \\
582.40 \\
1.6529\end{array}$ & $\begin{array}{r}12.3474 \\
588.17 \\
1.6565\end{array}$ & $\begin{array}{r}12.5000 \\
593.96 \\
1.6600\end{array}$ & $\begin{array}{r}12.6526 \\
599.76 \\
1.6635\end{array}$ & $\begin{array}{r}12.8052 \\
505.58 \\
1.6670\end{array}$ & $\begin{array}{r}12.9578 \\
611.41 \\
1.6705\end{array}$ \\
\hline 36 & $\begin{array}{l}V \\
H \\
S\end{array}$ & $\begin{array}{r}10.4340 \\
565.17 \\
1.6366\end{array}$ & $\begin{array}{r}10.5698 \\
570.89 \\
1.6403\end{array}$ & $\begin{array}{r}10.7055 \\
576.63 \\
1.6440\end{array}$ & $\begin{array}{r}10.8412 \\
582.39 \\
1.6476\end{array}$ & $\begin{array}{r}10.9769 \\
588.16 \\
1.6512\end{array}$ & $\begin{array}{r}11.1126 \\
593.95 \\
1.6547\end{array}$ & $\begin{array}{r}11.2483 \\
599.75 \\
1.6582\end{array}$ & $\begin{array}{r}11.3840 \\
605.57 \\
1.6617\end{array}$ & $\begin{array}{r}11.5196 \\
611.41 \\
1.6652\end{array}$ \\
\hline 40 & $\begin{array}{l}V \\
H \\
S\end{array}$ & $\begin{array}{l}9.3918 \\
565.16 \\
1.6319\end{array}$ & $\begin{array}{l}9.5140 \\
570.88 \\
1.6356\end{array}$ & $\begin{array}{l}9.6362 \\
576.62 \\
1.5392\end{array}$ & $\begin{array}{l}9.7584 \\
582.38 \\
1.6428\end{array}$ & $\begin{array}{l}9.8805 \\
588.15 \\
1.6464\end{array}$ & $\begin{array}{r}10.0027 \\
593.94 \\
1.6500\end{array}$ & $\begin{array}{r}10.1248 \\
599.74 \\
1.6535\end{array}$ & $\begin{array}{r}10.2470 \\
605.56 \\
1.6570\end{array}$ & $\begin{array}{r}10.3691 \\
611.40 \\
1.6604\end{array}$ \\
\hline 44 & $\begin{array}{l}V \\
H \\
S\end{array}$ & $\begin{array}{l}8.5391 \\
565.15 \\
1.6276\end{array}$ & $\begin{array}{l}8.6502 \\
570.87 \\
1.6313\end{array}$ & $\begin{array}{l}8.7613 \\
576.61 \\
1.6349\end{array}$ & $\begin{array}{l}8.8724 \\
582.37 \\
1.6386\end{array}$ & $\begin{array}{l}8.9835 \\
588.14 \\
1.6421\end{array}$ & $\begin{array}{l}9.0946 \\
593.93 \\
1.6457\end{array}$ & $\begin{array}{l}9.2056 \\
599.73 \\
2.6492\end{array}$ & $\begin{array}{l}9.3167 \\
605.55 \\
1.6527\end{array}$ & $\begin{array}{l}9.4278 \\
611.39 \\
1.6561\end{array}$ \\
\hline 48 & $\begin{array}{l}V \\
H \\
S\end{array}$ & $\begin{array}{l}7.8285 \\
565.14 \\
1.6237\end{array}$ & $\begin{array}{l}7.9304 \\
570.86 \\
1.6274\end{array}$ & $\begin{array}{l}8.0322 \\
576.50 \\
1.6310\end{array}$ & $\begin{array}{l}8.1341 \\
582.36 \\
1.6346\end{array}$ & $\begin{array}{l}8.2359 \\
588.13 \\
1.6382\end{array}$ & $\begin{array}{l}8.3378 \\
593.92 \\
1.6418\end{array}$ & $\begin{array}{l}8.4396 \\
599.73 \\
1.6453\end{array}$ & $\begin{array}{l}8.5 .415 \\
605.55 \\
1.6488\end{array}$ & $\begin{array}{l}8.6433 \\
611.38 \\
1.652=\end{array}$ \\
\hline 52 & $\begin{array}{l}V \\
H \\
S\end{array}$ & $\begin{array}{l}7.2272 \\
565.13 \\
1.6201\end{array}$ & $\begin{array}{l}7.3213 \\
570.85 \\
1.6238\end{array}$ & $\begin{array}{l}7.4153 \\
576.59 \\
1.6274\end{array}$ & $\begin{array}{l}7.5094 \\
582.35 \\
1.6310\end{array}$ & $\begin{array}{l}7.6034 \\
588.12 \\
1.6346\end{array}$ & $\begin{array}{l}7.6975 \\
593.91 \\
1.6382\end{array}$ & $\begin{array}{l}7.7915 \\
599.72 \\
1.6417\end{array}$ & $\begin{array}{l}7.8855 \\
605.54 \\
1.6452\end{array}$ & $\begin{array}{l}7.9795 \\
611.38 \\
1.6480\end{array}$ \\
\hline
\end{tabular}




\begin{tabular}{|c|c|c|c|c|c|c|c|c|c|c|}
\hline \multirow{2}{*}{\multicolumn{2}{|c|}{$\begin{array}{l}\text { PRESSURE } \\
\text { PSIA }\end{array}$}} & \multicolumn{8}{|c|}{ TEMPERATURE } & \multirow[b]{2}{*}{1240} \\
\hline & & 1080 & 1100 & 1120 & 1140 & 1160 & 1180 & 1200 & 1220 & \\
\hline 56 & $\begin{array}{l}V \\
H \\
S\end{array}$ & $\begin{array}{l}6.7118 \\
565.112 \\
1.6167\end{array}$ & $\begin{array}{l}6.7992 \\
570.84 \\
1.6204\end{array}$ & $\begin{array}{l}6.8865 \\
575.58 \\
1.6241\end{array}$ & $\begin{array}{l}6.9739 \\
582.34 \\
1.6277\end{array}$ & $\begin{array}{l}7.0612 \\
588.11 \\
1.6313\end{array}$ & $\begin{array}{l}7.1486 \\
593.90 \\
1.6348\end{array}$ & $\begin{array}{l}7.2359 \\
599.71 \\
1.6384\end{array}$ & $\begin{array}{l}7.3232 \\
605.53 \\
1.6418\end{array}$ & $\begin{array}{l}7.4106 \\
611.37 \\
1.6453\end{array}$ \\
\hline 60 & $\begin{array}{l}V \\
H \\
S\end{array}$ & $\begin{array}{l}6.2651 \\
565.11 \\
1.6136\end{array}$ & $\begin{array}{l}6.3467 \\
570.83 \\
1.6173\end{array}$ & $\begin{array}{l}6.4283 \\
576.57 \\
1.6210\end{array}$ & $\begin{array}{l}6.5098 \\
582.33 \\
1.6246\end{array}$ & $\begin{array}{l}6.5914 \\
588.10 \\
1.6282\end{array}$ & $\begin{array}{l}6.6729 \\
593.89 \\
1.6317\end{array}$ & $\begin{array}{l}6.7544 \\
599.70 \\
1.6353\end{array}$ & $\begin{array}{l}6.8360 \\
605.52 \\
1.6387\end{array}$ & $\begin{array}{l}6.9175 \\
611.36 \\
1.6422\end{array}$ \\
\hline $7 n$ & $\begin{array}{l}V \\
H \\
S\end{array}$ & $\begin{array}{l}5.3718 \\
565.08 \\
1.6067\end{array}$ & $\begin{array}{l}5.4417 \\
570.80 \\
1.6104\end{array}$ & $\begin{array}{l}5.5117 \\
576.55 \\
1.6140\end{array}$ & $\begin{array}{l}5.5817 \\
582.30 \\
1.6177\end{array}$ & $\begin{array}{l}5.6516 \\
588.08 \\
1.6212\end{array}$ & $\begin{array}{l}5.7215 \\
593.87 \\
1.6248\end{array}$ & $\begin{array}{l}5.7915 \\
599.68 \\
1.6283\end{array}$ & $\begin{array}{l}5.8614 \\
605.50 \\
1.6318\end{array}$ & $\begin{array}{l}5.9313 \\
611.34 \\
1.6352\end{array}$ \\
\hline 80 & $\begin{array}{l}V \\
H \\
S\end{array}$ & $\begin{array}{l}4.7018 \\
565.05 \\
1.6007\end{array}$ & $\begin{array}{l}4.7630 \\
570.78 \\
1.6044\end{array}$ & $\begin{array}{l}4.3243 \\
576.52 \\
1.6080\end{array}$ & $\begin{array}{l}4.8855 \\
582.28 \\
1.6116\end{array}$ & $\begin{array}{l}4.9468 \\
588.06 \\
1.6152\end{array}$ & $\begin{array}{l}5.0080 \\
593.85 \\
1.6188\end{array}$ & $\begin{array}{l}5.0692 \\
599.66 \\
1.6223\end{array}$ & $\begin{array}{l}5.1304 \\
505.48 \\
1.6258\end{array}$ & $\begin{array}{l}5.1917 \\
611.33 \\
1.6292\end{array}$ \\
\hline 90 & $\begin{array}{l}V \\
H \\
S\end{array}$ & $\begin{array}{l}4.1806 \\
565.02 \\
1.5954\end{array}$ & $\begin{array}{l}4.2351 \\
570.75 \\
1.5990\end{array}$ & $\begin{array}{l}4.2896 \\
576.49 \\
1.5027\end{array}$ & $\begin{array}{l}4.3441 \\
582.26 \\
1.6063\end{array}$ & $\begin{array}{l}4.3986 \\
588.03 \\
1.6099\end{array}$ & $\begin{array}{l}4.4530 \\
593.83 \\
1.6135\end{array}$ & $\begin{array}{l}4.5075 \\
599.64 \\
1.6170\end{array}$ & $\begin{array}{l}4.5619 \\
605.47 \\
1.6205\end{array}$ & $\begin{array}{l}4.6164 \\
611.31 \\
1.6239\end{array}$ \\
\hline 100 & $\begin{array}{l}V \\
H \\
S\end{array}$ & $\begin{array}{l}3.7637 \\
565.00 \\
1.5906\end{array}$ & $\begin{array}{l}3.8128 \\
570.72 \\
1.5943\end{array}$ & $\begin{array}{l}3.3619 \\
575.47 \\
1.5979\end{array}$ & $\begin{array}{l}3.9110 \\
582.23 \\
1.6016\end{array}$ & $\begin{array}{l}3.9600 \\
588.01 \\
1.6052\end{array}$ & $\begin{array}{l}4.0091 \\
593.81 \\
1.5087\end{array}$ & $\begin{array}{l}4.0581 \\
599.62 \\
1.6122\end{array}$ & $\begin{array}{l}4.1071 \\
605.45 \\
1.6157\end{array}$ & $\begin{array}{l}4.1562 \\
611.29 \\
1.6192\end{array}$ \\
\hline 110 & $\begin{array}{l}V \\
H \\
S\end{array}$ & $\begin{array}{l}3.4226 \\
564.97 \\
1.5863\end{array}$ & $\begin{array}{l}3.4673 \\
570.70 \\
1.5900\end{array}$ & $\begin{array}{l}3.5119 \\
576.44 \\
1.5936\end{array}$ & $\begin{array}{l}3.5566 \\
582.21 \\
1.5973\end{array}$ & $\begin{array}{l}3.6012 \\
587.99 \\
1.6008\end{array}$ & $\begin{array}{l}3.6458 \\
593.79 \\
1.6044\end{array}$ & $\begin{array}{l}3.6904 \\
599.60 \\
1.6079\end{array}$ & $\begin{array}{l}3.7350 \\
605.43 \\
1.6114\end{array}$ & $\begin{array}{l}3.7796 \\
611.27 \\
1.6149\end{array}$ \\
\hline 120 & $\begin{array}{l}V \\
H \\
S\end{array}$ & $\begin{array}{l}3.1384 \\
564.94 \\
1.5823\end{array}$ & $\begin{array}{l}3.1793 \\
570.67 \\
1.5860\end{array}$ & $\begin{array}{l}3.2203 \\
576.42 \\
1.5897\end{array}$ & $\begin{array}{l}3.2612 \\
582.18 \\
1.5933\end{array}$ & $\begin{array}{l}3.3022 \\
587.97 \\
1.5969\end{array}$ & $\begin{array}{l}3.3431 \\
593.76 \\
1.6005\end{array}$ & $\begin{array}{l}3.3840 \\
599.58 \\
1.6040\end{array}$ & $\begin{array}{l}3.4249 \\
605.41 \\
1.6075\end{array}$ & $\begin{array}{l}3.4658 \\
611.25 \\
1.6109\end{array}$ \\
\hline 130 & $\begin{array}{l}V \\
H \\
S\end{array}$ & $\begin{array}{l}2.8979 \\
564.91 \\
1.5787\end{array}$ & $\begin{array}{l}2.9357 \\
570.64 \\
1.5824\end{array}$ & $\begin{array}{l}2.9735 \\
576.39 \\
1.5861\end{array}$ & $\begin{array}{l}3.0113 \\
582.16 \\
1.5897\end{array}$ & $\begin{array}{l}3.0491 \\
587.94 \\
1.5933\end{array}$ & $\begin{array}{l}3.0869 \\
593.74 \\
1.5969\end{array}$ & $\begin{array}{l}3.1247 \\
599.56 \\
1.6004\end{array}$ & $\begin{array}{l}3.1625 \\
605.39 \\
1.6039\end{array}$ & $\begin{array}{l}3.2003 \\
611.23 \\
1.6073\end{array}$ \\
\hline 140 & $\begin{array}{l}V \\
H \\
S\end{array}$ & $\begin{array}{l}2.6917 \\
564.88 \\
1.5754\end{array}$ & $\begin{array}{l}2.7268 \\
570.62 \\
1.5791\end{array}$ & $\begin{array}{l}2.7620 \\
576.37 \\
1.5827\end{array}$ & $\begin{array}{l}2.7971 \\
582.14 \\
1.5864\end{array}$ & $\begin{array}{l}2.8323 \\
587.92 \\
1.5899\end{array}$ & $\begin{array}{l}2.8674 \\
593.72 \\
1.5935\end{array}$ & $\begin{array}{l}2.9025 \\
599.54 \\
1.5970\end{array}$ & $\begin{array}{l}2.9376 \\
605.37 \\
1.6005\end{array}$ & $\begin{array}{l}2.9727 \\
611.22 \\
1.6040\end{array}$ \\
\hline 150 & $\begin{array}{l}V \\
H \\
S\end{array}$ & $\begin{array}{l}2.5130 \\
564.86 \\
1.5722\end{array}$ & $\begin{array}{l}2.5458 \\
570.59 \\
1.5759\end{array}$ & $\begin{array}{l}2.5787 \\
576.34 \\
1.5796\end{array}$ & $\begin{array}{l}2.6115 \\
582.11 \\
1.5832\end{array}$ & $\begin{array}{l}2.6443 \\
587.90 \\
1.5868\end{array}$ & $\begin{array}{l}2.6771 \\
593.70 \\
1.5904\end{array}$ & $\begin{array}{l}2.7099 \\
599.52 \\
1.5939\end{array}$ & $\begin{array}{l}2.7427 \\
605.35 \\
1.5974\end{array}$ & $\begin{array}{l}2.7755 \\
611.20 \\
1.6008\end{array}$ \\
\hline
\end{tabular}




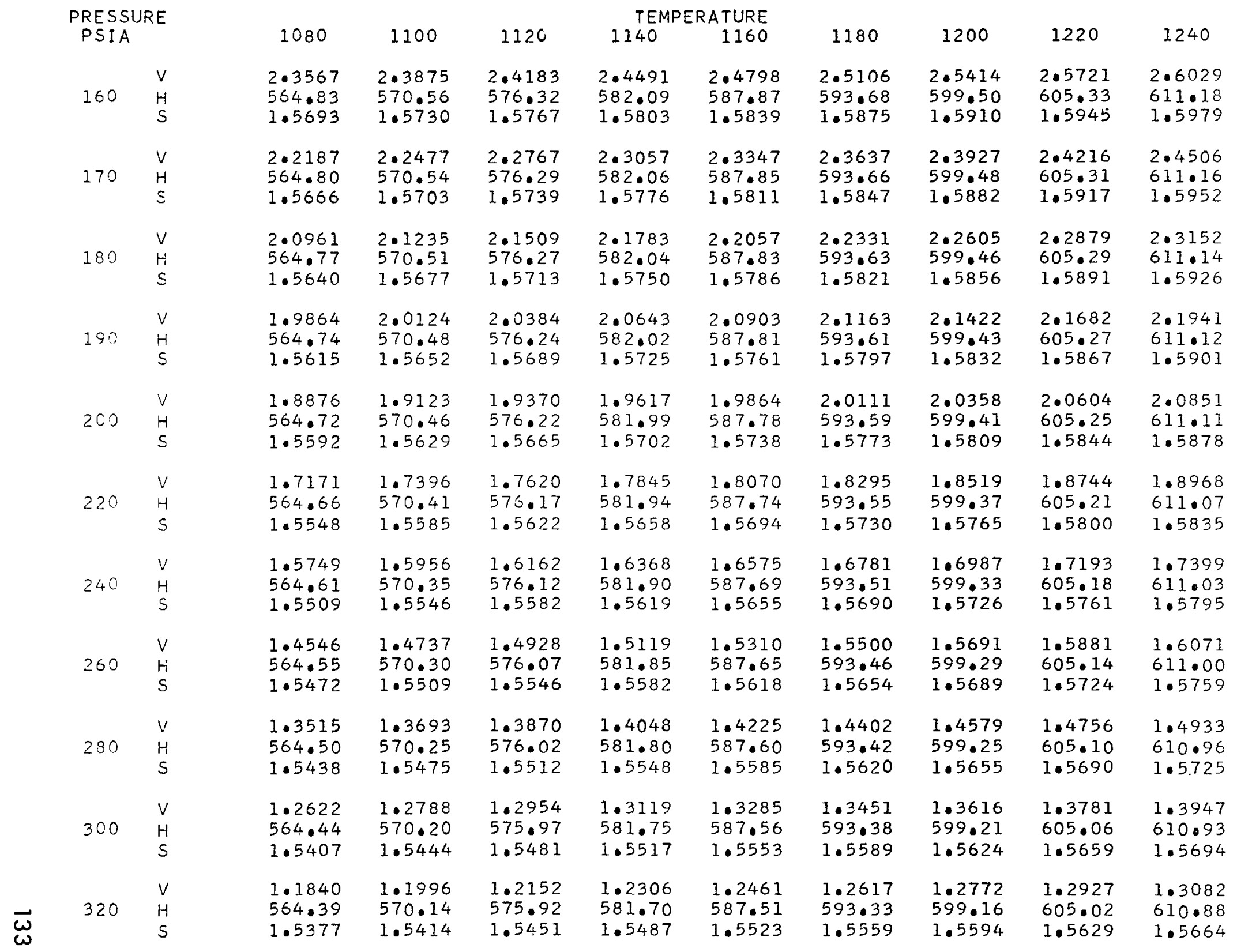


1.1149

564.32

1.1296
570.08

$340 \quad \begin{aligned} & \mathrm{H} \\ & \mathrm{S}\end{aligned}$

1.5349

1.5386

1.0536

360

1.5323

1.0675

570.03
1.5360

0.9987

1.0119

1.5298

1.5335

1120
1.1442
575.86
1.5423
1.0813
575.81
1.5397

TEMPERATURE

400

\subsection{3}

564.16

0.9618

569.92

1.5275

1.5312

0.9046

564.10

0.9166

569.87

1.5252

1. 5289

1.0250

575.76

1.5372

0.9743

575.71

1.5349

0.9285

575.66

0.8640

564.05

0.8754

569.82

2. 5326

0.9868

575.61

1.5305

0.8269

563.99

0.8378

569.77

0.8487

575.56

1.5284

0.7929

563.94

0.8034

569.71

0.8138

575.51

1.5264

0.7717

0.7817

0.7616

563.88

569.66

1.5209

575.46

1.5245

0.7424

0.7327

563.83

569.61

0.7521

575.41

1.5227

0.7060

563.77

0.7153
569.56

0.7247

575.36

1.5173

1.5210

1140

1.1589
581.65

581.65
1.5460

1. 0952

581.60

1.5433

1.0382

581.56

1.5409

0.9868

581.51

I. 5385

0.9404

581.46

1. 5362

0.8982

581.41

1. 5341

0.8596

581.36

1. 5321

0.8243

581.32

1. 5301

0.7918

581.27

1. 5282

0.7618

581.22

1. 5264

0.7340

581.17

1.5246

\section{0}

1.1735

587.46

1.5496

1.1090

587.41

1.5469

1.0513

587.37

1. 5445

0.9993

587.32

1.5421

0.9523

587.28

1. 5399

0.0096

587.23

1.5377

0.8706

587.19

1.5357

0.8348

587.14

1. 3337

0.8019

587.10

1.5318

0.7715

587.05

1.5300

0.7433

587.00

1.5282

\section{0}

1.1382

593.28

1.5531

1.1229

593.24

1. 5505

1.0644

593.20

I. 5480

1.0118

593.15

1.5457

0.9642

593.11

1.5434

0.9210

593.07

1. 5413

0.8815

593.02

1.5392

0.8452

592.98

1.5373

0.8119

592.94

1.5354

0.7811

592.89

1.5336

0.7527

592.85

1. 5318

\section{0}

1.2028

599.12

1.5567

1. 1367

599.08

1.5541

1.0776

599.04

1.5516

1. 0243

599.00

1.5492

0.9761

598.96

1. 5470

0.9323

598.92

1.5448

0.8923

598.88

1. 5428

0.8557

598.83

1.5408

0.8220

598.79

1.5389

0.7908

598.75

1.5371

0.7620

598.71

1. 5354
1220

1.2174

604.98

1.5602

1.1505

604.94

1.5576

1.0907

604.90

1.5551

1.0368

604.86

1.5527

0.9880

604.82

1.5505

0.9437

604.78

1.5483

0.9032

604.74

1.5463

0.8661

604.70

1.5443

0.8320

604.66

1.5425

0.8005

604.63

1.5406

0.7713

604.59

1.5389

1240

I. 2321 610.84

1.5636

1.1644 610.81

1.5610

1.1038

610.77

1.5586

1.0492

610.73

1. 5562

0.9999

610.70

1.5540

0.9551

610.66 


\begin{tabular}{|c|c|c|c|c|c|c|c|c|c|c|}
\hline \multirow{2}{*}{\multicolumn{2}{|c|}{$\begin{array}{l}\text { PRESSURE } \\
\text { PSIA }\end{array}$}} & \multicolumn{8}{|c|}{ TEMPERATURE } & \multirow{2}{*}{1240} \\
\hline & & 1080 & 1100 & 1120 & 1140 & 1160 & 1180 & 1200 & 1220 & \\
\hline 560 & $\begin{array}{l}V \\
H \\
S\end{array}$ & $\begin{array}{l}0.6811 \\
563.72 \\
1.5119\end{array}$ & $\begin{array}{l}0.6901 \\
569.50 \\
1.5156\end{array}$ & $\begin{array}{l}0.5992 \\
575.31 \\
1.5193\end{array}$ & $\begin{array}{l}0.7082 \\
581.12 \\
1.5229\end{array}$ & $\begin{array}{l}0.7172 \\
586.96 \\
1.5266\end{array}$ & $\begin{array}{l}0.7262 \\
592.81 \\
1.5301\end{array}$ & $\begin{array}{l}0.7352 \\
598.67 \\
1.5337\end{array}$ & $\begin{array}{l}0.7442 \\
604.55 \\
1.5372\end{array}$ & $\begin{array}{l}0.7532 \\
610.44 \\
1.5407\end{array}$ \\
\hline 580 & $\begin{array}{l}V \\
H \\
S\end{array}$ & $\begin{array}{l}0.6580 \\
563.66 \\
1.5102\end{array}$ & $\begin{array}{l}0.6667 \\
569.45 \\
1.5140\end{array}$ & $\begin{array}{l}0.6754 \\
575.26 \\
1.5176\end{array}$ & $\begin{array}{l}0.6842 \\
581.08 \\
1.5213\end{array}$ & $\begin{array}{l}0.6929 \\
586.91 \\
1.5249\end{array}$ & $\begin{array}{l}0.7016 \\
592.76 \\
1.5285\end{array}$ & $\begin{array}{l}0.7103 \\
598.63 \\
1.5321\end{array}$ & $\begin{array}{l}0.7190 \\
604.51 \\
1.5356\end{array}$ & $\begin{array}{l}0.7276 \\
610.40 \\
1.5391\end{array}$ \\
\hline 600 & $\begin{array}{l}V \\
H \\
S\end{array}$ & $\begin{array}{l}0.6364 \\
563.61 \\
1.5086\end{array}$ & $\begin{array}{l}0.6449 \\
569.40 \\
1.5124\end{array}$ & $\begin{array}{l}0.6533 \\
575.21 \\
1.5161\end{array}$ & $\begin{array}{l}0.6617 \\
581.03 \\
1.5197\end{array}$ & $\begin{array}{l}0.6702 \\
586.87 \\
1.5234\end{array}$ & $\begin{array}{l}0.6786 \\
592.72 \\
1.5269\end{array}$ & $\begin{array}{l}0.6870 \\
598.59 \\
1.5305\end{array}$ & $\begin{array}{l}0.6954 \\
604.47 \\
1.5340\end{array}$ & $\begin{array}{l}0.7038 \\
610.37 \\
1.5375\end{array}$ \\
\hline 620 & $\begin{array}{l}V \\
H \\
S\end{array}$ & $\begin{array}{l}0.6162 \\
563.55 \\
1.5071\end{array}$ & $\begin{array}{l}0.6244 \\
569.35 \\
1.5108\end{array}$ & $\begin{array}{l}0.6326 \\
575.16 \\
1.5145\end{array}$ & $\begin{array}{l}0.6408 \\
530.98 \\
1.5182\end{array}$ & $\begin{array}{l}0.6489 \\
586.82 \\
1.5218\end{array}$ & $\begin{array}{l}0.6571 \\
592.68 \\
1.5254\end{array}$ & $\begin{array}{l}0.6652 \\
598.55 \\
1.5290\end{array}$ & $\begin{array}{l}0.6734 \\
604.43 \\
1.5325\end{array}$ & $\begin{array}{l}0.6815 \\
610.33 \\
1.5360\end{array}$ \\
\hline 640 & $\begin{array}{l}V \\
H \\
S\end{array}$ & $\begin{array}{l}0.5973 \\
563.50 \\
1.5056\end{array}$ & $\begin{array}{l}0.6052 \\
559.29 \\
1.5094\end{array}$ & $\begin{array}{l}0.5132 \\
575.11 \\
1.5131\end{array}$ & $\begin{array}{l}0.6211 \\
580.93 \\
1.5167\end{array}$ & $\begin{array}{l}0.6290 \\
586.78 \\
1.5203\end{array}$ & $\begin{array}{l}0.6369 \\
592.63 \\
1.5239\end{array}$ & $\begin{array}{l}0.6448 \\
598.51 \\
1.5275\end{array}$ & $\begin{array}{l}0.6527 \\
604.39 \\
1.5310\end{array}$ & $\begin{array}{l}0.6606 \\
610.29 \\
1.5345\end{array}$ \\
\hline 660 & $\begin{array}{l}V \\
H \\
S\end{array}$ & $\begin{array}{l}0.5795 \\
563.44 \\
1.5042\end{array}$ & $\begin{array}{l}0.5872 \\
569.24 \\
1.5079\end{array}$ & $\begin{array}{l}0.5949 \\
575.06 \\
1.5116\end{array}$ & $\begin{array}{l}0.6026 \\
580.89 \\
1.5153\end{array}$ & $\begin{array}{l}0.6103 \\
586.73 \\
1.5189\end{array}$ & $\begin{array}{l}0.6180 \\
592.59 \\
1.5225\end{array}$ & $\begin{array}{l}0.6257 \\
598.47 \\
1.5261\end{array}$ & $\begin{array}{l}0.6333 \\
604.35 \\
1.5296\end{array}$ & $\begin{array}{l}0.6410 \\
610.26 \\
1.5331\end{array}$ \\
\hline 680 & $\begin{array}{l}V \\
H \\
S\end{array}$ & $\begin{array}{l}0.5627 \\
563.39 \\
1.5028\end{array}$ & $\begin{array}{l}0.5702 \\
569.19 \\
1.5065\end{array}$ & $\begin{array}{l}0.5777 \\
575.01 \\
1.5102\end{array}$ & $\begin{array}{l}0.5852 \\
580.84 \\
1.5139\end{array}$ & $\begin{array}{l}0.5927 \\
586.69 \\
1.5175\end{array}$ & $\begin{array}{l}0.6001 \\
592.55 \\
1.5211\end{array}$ & $\begin{array}{l}0.6076 \\
598.42 \\
1.5247\end{array}$ & $\begin{array}{l}0.6151 \\
604.31 \\
1.5282\end{array}$ & $\begin{array}{l}0.6225 \\
610.22 \\
1.5317\end{array}$ \\
\hline 700 & $\begin{array}{l}V \\
H \\
S\end{array}$ & $\begin{array}{l}0.5469 \\
563.33 \\
1.5014\end{array}$ & $\begin{array}{l}0.5542 \\
569.14 \\
1.5052\end{array}$ & $\begin{array}{l}0.5615 \\
574.96 \\
1.5089\end{array}$ & $\begin{array}{l}0.5688 \\
580.79 \\
1.5125\end{array}$ & $\begin{array}{l}0.5761 \\
586.64 \\
1.5162\end{array}$ & $\begin{array}{l}0.5833 \\
592.51 \\
1.5198\end{array}$ & $\begin{array}{l}0.5906 \\
598.38 \\
1.5233\end{array}$ & $\begin{array}{l}0.5978 \\
604.28 \\
1.5268\end{array}$ & $\begin{array}{l}0.6051 \\
610.18 \\
1.5303\end{array}$ \\
\hline 720 & $\begin{array}{l}V \\
H \\
S\end{array}$ & $\begin{array}{l}0.5320 \\
563.28 \\
1.5001\end{array}$ & $\begin{array}{l}0.5391 \\
569.08 \\
1.5038\end{array}$ & $\begin{array}{l}0.5462 \\
57+.91 \\
1.5075\end{array}$ & $\begin{array}{l}0.5533 \\
580.74 \\
1.5112\end{array}$ & $\begin{array}{l}0.5604 \\
586.60 \\
1.5148\end{array}$ & $\begin{array}{l}0.5674 \\
592.46 \\
1.5184\end{array}$ & $\begin{array}{l}0.5745 \\
598.34 \\
1.5220\end{array}$ & $\begin{array}{l}0.5816 \\
604.24 \\
1.5255\end{array}$ & $\begin{array}{l}0.5886 \\
610.15 \\
1.5290\end{array}$ \\
\hline 740 & $\begin{array}{l}V \\
H \\
S\end{array}$ & $\begin{array}{l}0.5179 \\
563.22 \\
1.4988\end{array}$ & $\begin{array}{l}0.5248 \\
569.03 \\
1.5025\end{array}$ & $\begin{array}{l}0.5318 \\
574.86 \\
1.5063\end{array}$ & $\begin{array}{l}0.5386 \\
580.70 \\
1.5099\end{array}$ & $\begin{array}{l}0.5455 \\
586.55 \\
1.5136\end{array}$ & $\begin{array}{l}0.5524 \\
592.42 \\
1.5172\end{array}$ & $\begin{array}{l}0.5593 \\
598.30 \\
1.5207\end{array}$ & $\begin{array}{l}0.5662 \\
604.20 \\
1.5242\end{array}$ & $\begin{array}{l}0.5730 \\
610.11 \\
1.5277\end{array}$ \\
\hline 760 & $\begin{array}{l}V \\
H \\
S\end{array}$ & $\begin{array}{l}0.5046 \\
563.17 \\
1.4975\end{array}$ & $\begin{array}{l}0.5113 \\
568.98 \\
1.5013\end{array}$ & $\begin{array}{l}0.5180 \\
574.81 \\
1.5050\end{array}$ & $\begin{array}{l}0.5248 \\
580.65 \\
1.5087\end{array}$ & $\begin{array}{l}0.5315 \\
586.51 \\
1.5123\end{array}$ & $\begin{array}{l}0.5382 \\
592.38 \\
1.5159\end{array}$ & $\begin{array}{l}0.5449 \\
598.26 \\
1.5195\end{array}$ & $\begin{array}{l}0.5516 \\
604.16 \\
1.5230\end{array}$ & $\begin{array}{l}0.5583 \\
610.07 \\
1.5265\end{array}$ \\
\hline
\end{tabular}


TEMPERATURE

780

0.4985

563.11

568.93

0.5050

1140

1160

1180

1200

1220

1240

1.4963

1.5001

574.76

0.5116

0.5181

580.60

586.46

$0.4798 \quad 0.4863$

563.06

0.4863
568.88

1.5038

1.5074

1.5111

0.5247

0.5312

0.5378

0.5443

0.4927

574.71

0.4991

580.55

0.5055

1.5147

598.22

$604 \cdot 12$
1.5218

610.03

300

1.4989

1.3026

1.5063

1.5099

0.5119

592.29

0.5182

0.5246

598.18

604.08

1.5253

\section{$0.4684 \quad 0.4746$}

$563.00 \quad 568.82$

0.4809

0.4872

0.4934

574.66

580.51

586.37

0.4996

1.4977

1.5014

1.5051

1.5087

592.25

1.517

1.5206

0.5310

610000

1.5241

$\begin{array}{ll}0.4575 & 0.4636\end{array}$

$562.95 \quad 568.77$

0.4697

0.4758

0.4819

1.5123

0.5059

0.5121

0.5183

598.14

604.04

609096

574.61

520.46

586.32

0.4880

1.5159

1.5194

$1: 5220$

1.4966

1.5003

1. 5040

1.5076

592.20

0.4941

0.5002

0.5053

1.5112

604.00

609.92

$0.4590 \quad 0.4650$

0.4710

0.4769

1.5148

1.5183

1.5218

$\begin{array}{ll}0.4470 & 0.4530\end{array}$

1.4917

1.4954

$57 \div .56$

580.41

536.28

592.16

0.4829

0.4888

603.97

0.4943

1.5101

1.5137

1.5172

609.89

$\begin{array}{lllll}0.4371 & 0.4430 & 0.4488 & 0.4547 & 0.4505\end{array}$

562.84

0.4430
568.67

0.4663

1.4944

1.4981

1.5018

1.5054

592.12

0.4722

0.4780

603.93

1.5161

0.4838

1.5126

0.4676

0.4562

592.08

0.4619

597.97

603.89

586.19

1.5079

1. 5115

1.5151

$1.5007 \quad 1.5043$

0.4466

0.4521

0.4577

0.4410

592.03

597.93

503.85

1.5105

1.5140

0.4428

0.4482

0.4373

507.89

503.81

1.5130

1.5023

1.5059

1.5095

0.4391

0.4230

586.05

0.4284

591.95

0.4338

597.85

603.77

1.5120

1.5085

0.4304

0.4146

$\begin{array}{lll}580.13 & 586.01 & 591090 \\ 1.4966 & 1.5003 & 1.5039\end{array}$

0.4199

$\begin{array}{lll}580.13 & 586.01 & 591090 \\ 1.4966 & 1.5003 & 1.5039\end{array}$

0.4252

597.81

603.73

1.5110

1.5106

0.4633

609.78

1. 5175

0.2537

509.76

1.5165

0.4445

609070

1.5155

1.5075

$0 .<357$

609.67

1.5145 


\begin{tabular}{|c|c|c|c|c|c|c|c|c|c|c|}
\hline \multirow{2}{*}{\multicolumn{2}{|c|}{$\begin{array}{l}\text { PRESSURE } \\
\text { PSIA }\end{array}$}} & \multicolumn{8}{|c|}{ TEMPERATURE } & \multirow[b]{2}{*}{1240} \\
\hline & & 1080 & 1100 & 1120 & 1140 & 1160 & 1180 & 1200 & 1220 & \\
\hline 1000 & $\begin{array}{l}V \\
H \\
S\end{array}$ & $\begin{array}{l}0.3858 \\
562.51 \\
1.4845\end{array}$ & $\begin{array}{l}0.3910 \\
568.36 \\
1.4883\end{array}$ & $\begin{array}{l}0.3962 \\
574.21 \\
1.4920\end{array}$ & $\begin{array}{l}0.4014 \\
580.08 \\
1.4957\end{array}$ & $\begin{array}{l}0.4065 \\
585.96 \\
1.4993\end{array}$ & $\begin{array}{l}0.4117 \\
591.86 \\
1.5029\end{array}$ & $\begin{array}{l}0.4169 \\
597.77 \\
1.5065\end{array}$ & $\begin{array}{l}0.4220 \\
603.69 \\
1.5101\end{array}$ & $\begin{array}{l}0.4272 \\
609.63 \\
1.5136\end{array}$ \\
\hline 1020 & $\begin{array}{l}V \\
H \\
S\end{array}$ & $\begin{array}{l}0.3784 \\
562.46 \\
1.4836\end{array}$ & $\begin{array}{l}0.3835 \\
568.30 \\
1.4873\end{array}$ & $\begin{array}{l}0.3886 \\
574.16 \\
1.4910\end{array}$ & $\begin{array}{l}0.3937 \\
580.03 \\
1.4947\end{array}$ & $\begin{array}{l}0.3988 \\
585.92 \\
1.4984\end{array}$ & $\begin{array}{l}0.4039 \\
591.82 \\
1.5020\end{array}$ & $\begin{array}{l}0.4089 \\
597.73 \\
1.5056\end{array}$ & $\begin{array}{l}0.4140 \\
503.66 \\
1.5091\end{array}$ & $\begin{array}{l}0.4190 \\
609.59 \\
1.5126\end{array}$ \\
\hline 1040 & $\begin{array}{l}V \\
H \\
S\end{array}$ & $\begin{array}{l}0.3713 \\
562.41 \\
1.4826\end{array}$ & $\begin{array}{l}0.3763 \\
568.25 \\
1.4864\end{array}$ & $\begin{array}{l}0.3813 \\
57+11 \\
1.4901\end{array}$ & $\begin{array}{l}0.3863 \\
579.99 \\
1.4938\end{array}$ & $\begin{array}{l}0.3913 \\
585.87 \\
1.4975\end{array}$ & $\begin{array}{l}0.3963 \\
591.78 \\
1.5011\end{array}$ & $\begin{array}{l}0.4013 \\
597.69 \\
1.5047\end{array}$ & $\begin{array}{l}0.4062 \\
603.62 \\
1.5082\end{array}$ & $\begin{array}{l}0.4112 \\
609.56 \\
1.5117\end{array}$ \\
\hline 1060 & $\begin{array}{l}V \\
H \\
S\end{array}$ & $\begin{array}{l}0.3645 \\
562.35 \\
1.4817\end{array}$ & $\begin{array}{l}0.3694 \\
568.20 \\
1.4855\end{array}$ & $\begin{array}{l}0.3743 \\
574.06 \\
1.4892\end{array}$ & $\begin{array}{l}0.3792 \\
579.94 \\
1.4929\end{array}$ & $\begin{array}{l}0.3841 \\
585.83 \\
1.4966\end{array}$ & $\begin{array}{l}0.3390 \\
591.73 \\
1.5002\end{array}$ & $\begin{array}{l}0.3939 \\
597.65 \\
1.5038\end{array}$ & $\begin{array}{l}0.3988 \\
603.58 \\
1.5073\end{array}$ & $\begin{array}{l}0.4037 \\
609.52 \\
1.5108\end{array}$ \\
\hline 1080 & $\begin{array}{l}V \\
H \\
S\end{array}$ & $\begin{array}{l}0.3579 \\
562.30 \\
1.4808\end{array}$ & $\begin{array}{l}0.3628 \\
568.15 \\
1.4846\end{array}$ & $\begin{array}{l}0.3676 \\
574.01 \\
1.4883\end{array}$ & $\begin{array}{l}0.3724 \\
579.89 \\
1.4920\end{array}$ & $\begin{array}{l}0.3772 \\
585.78 \\
1.4957\end{array}$ & $\begin{array}{l}0.3820 \\
591.69 \\
1.4993\end{array}$ & $\begin{array}{l}0.3868 \\
597.61 \\
1.5029\end{array}$ & $\begin{array}{l}0.3916 \\
603.54 \\
1.5064\end{array}$ & $\begin{array}{l}0.3964 \\
609.48 \\
1.5099\end{array}$ \\
\hline 1100 & $\begin{array}{l}V \\
H \\
S\end{array}$ & $\begin{array}{l}0.3516 \\
562.24 \\
1.4799\end{array}$ & $\begin{array}{l}0.3563 \\
568.10 \\
1.4837\end{array}$ & $\begin{array}{l}0.3611 \\
573.96 \\
1.4874\end{array}$ & $\begin{array}{l}0.3658 \\
579.85 \\
1.4911\end{array}$ & $\begin{array}{l}0.3705 \\
585.74 \\
1.4948\end{array}$ & $\begin{array}{l}0.3753 \\
591.65 \\
1.4984\end{array}$ & $\begin{array}{l}0.3800 \\
597.57 \\
1.5020\end{array}$ & $\begin{array}{l}0.3847 \\
603.50 \\
1.5055\end{array}$ & $\begin{array}{l}0.3894 \\
609.45 \\
1.5091\end{array}$ \\
\hline 1120 & $\begin{array}{l}V \\
H \\
S\end{array}$ & $\begin{array}{l}0.3455 \\
562.19 \\
1.4791\end{array}$ & $\begin{array}{l}0.3501 \\
568.05 \\
1.4828\end{array}$ & $\begin{array}{l}0.3548 \\
573.92 \\
1.4866\end{array}$ & $\begin{array}{l}0.3595 \\
579.80 \\
1.4903\end{array}$ & $\begin{array}{l}0.3641 \\
585.69 \\
1.4939\end{array}$ & $\begin{array}{l}0.3688 \\
591.60 \\
1.4975\end{array}$ & $\begin{array}{l}0.3734 \\
597.53 \\
1.5011\end{array}$ & $\begin{array}{l}0.3780 \\
603.46 \\
1.5047\end{array}$ & $\begin{array}{l}0.3826 \\
609.41 \\
1.5082\end{array}$ \\
\hline 1140 & $\begin{array}{l}V \\
H \\
S\end{array}$ & $\begin{array}{l}0.3396 \\
562.14 \\
1.4782\end{array}$ & $\begin{array}{l}0.3442 \\
567.99 \\
1.4820\end{array}$ & $\begin{array}{l}0.3488 \\
573.87 \\
1.4857\end{array}$ & $\begin{array}{l}0.3533 \\
579.75 \\
1.4894\end{array}$ & $\begin{array}{l}0.3579 \\
585.65 \\
1.4931\end{array}$ & $\begin{array}{l}0.3625 \\
591.56 \\
1.4967\end{array}$ & $\begin{array}{l}0.3670 \\
597.49 \\
1.5003\end{array}$ & $\begin{array}{l}0.3716 \\
603.42 \\
1.5038\end{array}$ & $\begin{array}{l}0.3761 \\
609.37 \\
1.5074\end{array}$ \\
\hline 1160 & $\begin{array}{l}V \\
H \\
S\end{array}$ & $\begin{array}{l}0.3339 \\
562.08 \\
1.4774\end{array}$ & $\begin{array}{l}0.3384 \\
567.94 \\
1.4811\end{array}$ & $\begin{array}{l}0.3429 \\
573.82 \\
1.4849\end{array}$ & $\begin{array}{l}0.3474 \\
579.70 \\
1.4886\end{array}$ & $\begin{array}{l}0.3519 \\
585.60 \\
1.4922\end{array}$ & $\begin{array}{l}0.3564 \\
591.52 \\
1.4959\end{array}$ & $\begin{array}{l}0.3609 \\
597.45 \\
1.4995\end{array}$ & $\begin{array}{l}0.3654 \\
603.38 \\
1.5030\end{array}$ & $\begin{array}{l}0.3698 \\
609.34 \\
1.5065\end{array}$ \\
\hline 1180 & $\begin{array}{l}V \\
H \\
S\end{array}$ & $\begin{array}{l}0.3284 \\
562.03 \\
1.4765\end{array}$ & $\begin{array}{l}0.3328 \\
567.89 \\
1.4803\end{array}$ & $\begin{array}{l}0.3373 \\
573.77 \\
1.4841\end{array}$ & $\begin{array}{l}0.3417 \\
579.66 \\
1.4878\end{array}$ & $\begin{array}{l}0.3461 \\
585.56 \\
1.4914\end{array}$ & $\begin{array}{l}0.3506 \\
591.48 \\
1.4950\end{array}$ & $\begin{array}{l}0.3550 \\
597.40 \\
1.4986\end{array}$ & $\begin{array}{l}0.3594 \\
603.35 \\
1.5022\end{array}$ & $\begin{array}{l}0.3638 \\
609.30 \\
1.5057\end{array}$ \\
\hline 1200 & $\begin{array}{l}V \\
H \\
S\end{array}$ & $\begin{array}{l}0.3231 \\
561.98 \\
1.4757\end{array}$ & $\begin{array}{l}0.3274 \\
567.84 \\
1.4795\end{array}$ & $\begin{array}{l}0.3318 \\
573.72 \\
1.4832\end{array}$ & $\begin{array}{l}0.3362 \\
579.61 \\
1.4869\end{array}$ & $\begin{array}{l}0.3405 \\
585.52 \\
1.4906\end{array}$ & $\begin{array}{l}0.3449 \\
591.43 \\
1.4942\end{array}$ & $\begin{array}{l}0.3492 \\
597.36 \\
1.4978\end{array}$ & $\begin{array}{l}0.3536 \\
603.31 \\
1.5014\end{array}$ & $\begin{array}{l}0.3579 \\
609.26 \\
1.5049\end{array}$ \\
\hline
\end{tabular}




\begin{tabular}{|c|c|c|c|c|c|c|c|c|c|c|}
\hline 1220 & $\begin{array}{l}V \\
H \\
S\end{array}$ & $\begin{array}{l}0.3179 \\
561.92 \\
1.4749\end{array}$ & $\begin{array}{l}0.3222 \\
567.79 \\
1.4787\end{array}$ & $\begin{array}{l}0.3265 \\
573.67 \\
1.4824\end{array}$ & $\begin{array}{l}0.3308 \\
579.56 \\
1.4862\end{array}$ & $\begin{array}{l}0.3351 \\
585.47 \\
1.4898\end{array}$ & $\begin{array}{l}0.3394 \\
591.39 \\
1.4934\end{array}$ & $\begin{array}{l}0.3437 \\
597.32 \\
1.4970\end{array}$ & $\begin{array}{l}0.3479 \\
603.27 \\
1.5006\end{array}$ & $\begin{array}{l}0.3522 \\
609.23 \\
1.5041\end{array}$ \\
\hline 1240 & $\begin{array}{l}V \\
H \\
S\end{array}$ & $\begin{array}{l}0.3129 \\
561.87 \\
1.4741\end{array}$ & $\begin{array}{l}0.3172 \\
567.74 \\
1.4779\end{array}$ & $\begin{array}{l}0.3214 \\
573.62 \\
1.4817\end{array}$ & $\begin{array}{l}0.3257 \\
579.52 \\
1.4854\end{array}$ & $\begin{array}{l}0.3299 \\
585.43 \\
1.4890\end{array}$ & $\begin{array}{l}0.3341 \\
591.35 \\
1.4927\end{array}$ & $\begin{array}{l}0.3383 \\
597.28 \\
1.4963\end{array}$ & $\begin{array}{l}0.3425 \\
603.23 \\
1.4998\end{array}$ & $\begin{array}{l}0.3467 \\
609.19 \\
1.5033\end{array}$ \\
\hline 1260 & $\begin{array}{l}V \\
H \\
S\end{array}$ & $\begin{array}{l}0.3081 \\
561.81 \\
1.4734\end{array}$ & $\begin{array}{l}0.3123 \\
567.69 \\
1.4771\end{array}$ & $\begin{array}{l}0.3165 \\
573.57 \\
1.4809\end{array}$ & $\begin{array}{l}0.3206 \\
579.47 \\
1.4846\end{array}$ & $\begin{array}{l}0.3248 \\
585.38 \\
1.4883\end{array}$ & $\begin{array}{l}0.3290 \\
591.30 \\
1.4919\end{array}$ & $\begin{array}{l}0.3331 \\
597.24 \\
1.4955\end{array}$ & $\begin{array}{l}0.3373 \\
603.19 \\
1.4990\end{array}$ & $\begin{array}{l}0.3414 \\
609.15 \\
1.5026\end{array}$ \\
\hline 1280 & $\begin{array}{l}V \\
H \\
S\end{array}$ & $\begin{array}{l}0.3034 \\
561.76 \\
1.4726\end{array}$ & $\begin{array}{l}0.3076 \\
567.63 \\
1.4764\end{array}$ & $\begin{array}{l}0.3117 \\
573.52 \\
1.4801\end{array}$ & $\begin{array}{l}0.3158 \\
579.42 \\
1.4838\end{array}$ & $\begin{array}{l}0.3199 \\
585.34 \\
1.4875\end{array}$ & $\begin{array}{l}0.3240 \\
591.26 \\
1.4911\end{array}$ & $\begin{array}{l}0.3281 \\
597.20 \\
1.4947\end{array}$ & $\begin{array}{l}0.3322 \\
603.15 \\
1.4983\end{array}$ & $\begin{array}{l}0.2362 \\
609.12 \\
1.5018\end{array}$ \\
\hline 1300 & $\begin{array}{l}V \\
H \\
S\end{array}$ & $\begin{array}{l}0.2989 \\
561.71 \\
1.4718\end{array}$ & $\begin{array}{l}0.3030 \\
567.58 \\
1.4756\end{array}$ & $\begin{array}{l}0.3070 \\
573.47 \\
1.4794\end{array}$ & $\begin{array}{l}0.3111 \\
579.38 \\
1.4831\end{array}$ & $\begin{array}{l}0.3151 \\
585.29 \\
1.4868\end{array}$ & $\begin{array}{l}0.3192 \\
591.22 \\
1.4904\end{array}$ & $\begin{array}{l}0.3232 \\
597.16 \\
1.4940\end{array}$ & $\begin{array}{l}0.3272 \\
603.11 \\
1.4975\end{array}$ & $\begin{array}{l}0.3312 \\
609.08 \\
1.5011\end{array}$ \\
\hline 1320 & $\begin{array}{l}V \\
H \\
S\end{array}$ & $\begin{array}{l}0.2945 \\
561.65 \\
1.4711\end{array}$ & $\begin{array}{l}0.2985 \\
567.53 \\
1.4749\end{array}$ & $\begin{array}{l}0.3025 \\
573.42 \\
1.4786\end{array}$ & $\begin{array}{l}0.3065 \\
579.33 \\
1.4823\end{array}$ & $\begin{array}{l}0.3105 \\
585.25 \\
1.4860\end{array}$ & $\begin{array}{l}0.3145 \\
591.18 \\
1.4996\end{array}$ & $\begin{array}{l}0.3184 \\
597.12 \\
1.4932\end{array}$ & $\begin{array}{l}0.3224 \\
603.07 \\
1.4968\end{array}$ & $\begin{array}{l}0.3264 \\
602.04 \\
1.5003\end{array}$ \\
\hline 1340 & $\begin{array}{l}V \\
H \\
S\end{array}$ & $\begin{array}{l}0.2902 \\
561.60 \\
1.4704\end{array}$ & $\begin{array}{l}0.2942 \\
567.48 \\
1.4741\end{array}$ & $\begin{array}{l}0.2981 \\
573.38 \\
1.4779\end{array}$ & $\begin{array}{l}0.3021 \\
579.28 \\
1.4816\end{array}$ & $\begin{array}{l}0.3060 \\
585.20 \\
1.4853\end{array}$ & $\begin{array}{l}0.3099 \\
591.13 \\
1.4889\end{array}$ & $\begin{array}{l}0.3139 \\
597.08 \\
1.4925\end{array}$ & $\begin{array}{l}0.3178 \\
603.04 \\
1.4961\end{array}$ & $\begin{array}{l}0.3217 \\
609.00 \\
1.4996\end{array}$ \\
\hline 1360 & $\begin{array}{l}V \\
H \\
S\end{array}$ & $\begin{array}{l}0.2861 \\
561.55 \\
1.4696\end{array}$ & $\begin{array}{l}0.2900 \\
567.43 \\
1.4734\end{array}$ & $\begin{array}{l}0.2939 \\
573.33 \\
1.4772\end{array}$ & $\begin{array}{l}0.2978 \\
579.24 \\
1.4809\end{array}$ & $\begin{array}{l}0.3017 \\
585.16 \\
1.4846\end{array}$ & $\begin{array}{l}0.3055 \\
591.09 \\
1.4382\end{array}$ & $\begin{array}{l}0.3094 \\
597.04 \\
1.4918\end{array}$ & $\begin{array}{l}0.3132 \\
603.00 \\
1.4954\end{array}$ & $\begin{array}{l}0.3171 \\
603.97 \\
1.4989\end{array}$ \\
\hline 1380 & $\begin{array}{l}V \\
H \\
S\end{array}$ & $\begin{array}{l}0.2821 \\
561.49 \\
1.4689\end{array}$ & $\begin{array}{l}0.2859 \\
567.38 \\
1.4727\end{array}$ & $\begin{array}{l}0.2898 \\
573.28 \\
1.4765\end{array}$ & $\begin{array}{l}0.2936 \\
579.19 \\
1.4802\end{array}$ & $\begin{array}{l}0.2974 \\
585.11 \\
1.4839\end{array}$ & $\begin{array}{l}0.3012 \\
591.05 \\
1.4875\end{array}$ & $\begin{array}{l}0.3051 \\
597.00 \\
1.4911\end{array}$ & $\begin{array}{l}0.3089 \\
602.96 \\
1.4947\end{array}$ & $\begin{array}{l}0.3127 \\
608.93 \\
1.4982\end{array}$ \\
\hline 1400 & $\begin{array}{l}V \\
H \\
S\end{array}$ & $\begin{array}{l}0.2782 \\
561.44 \\
1.4682\end{array}$ & $\begin{array}{l}0.2820 \\
567.33 \\
1.4720\end{array}$ & $\begin{array}{l}0.2858 \\
573.23 \\
1.4758\end{array}$ & $\begin{array}{l}0.2895 \\
579.14 \\
1.4795\end{array}$ & $\begin{array}{l}0.2933 \\
585.07 \\
1.4832\end{array}$ & $\begin{array}{l}0.2971 \\
591.01 \\
1.4868\end{array}$ & $\begin{array}{l}0.3008 \\
596.96 \\
1.4904\end{array}$ & $\begin{array}{l}0.3046 \\
602.92 \\
1.4940\end{array}$ & $\begin{array}{l}0.3083 \\
608.89 \\
1.4975\end{array}$ \\
\hline 1450 & $\begin{array}{l}V \\
H \\
S\end{array}$ & $\begin{array}{l}0.2689 \\
561.31 \\
1.4665\end{array}$ & $\begin{array}{l}0.2726 \\
567.20 \\
1.4703\end{array}$ & $\begin{array}{l}0.2762 \\
573.11 \\
1.4741\end{array}$ & $\begin{array}{l}0.2799 \\
579.02 \\
1.4778\end{array}$ & $\begin{array}{l}0.2835 \\
584.96 \\
1.4815\end{array}$ & $\begin{array}{l}0.2872 \\
590.90 \\
1.4851\end{array}$ & $\begin{array}{l}0.2908 \\
596.86 \\
1.4887\end{array}$ & $\begin{array}{l}0.2945 \\
602.82 \\
1.4923\end{array}$ & $\begin{array}{l}0.2981 \\
608.80 \\
1.4958\end{array}$ \\
\hline
\end{tabular}




\begin{tabular}{|c|c|c|c|c|c|c|c|c|c|c|}
\hline \multirow{2}{*}{\multicolumn{2}{|c|}{$\begin{array}{l}\text { PRESSURE } \\
\text { PSIA }\end{array}$}} & \multicolumn{8}{|c|}{ TEMPERATURE } & \multirow[b]{2}{*}{1240} \\
\hline & & 1080 & 1100 & 1120 & 1140 & 1160 & 1180 & 1200 & 1220 & \\
\hline 1500 & $\begin{array}{l}V \\
H \\
S\end{array}$ & $\begin{array}{l}0.2602 \\
561.17 \\
1.4648\end{array}$ & $\begin{array}{l}0.2638 \\
567.07 \\
1.4686\end{array}$ & $\begin{array}{l}0.2673 \\
572.98 \\
1.4724\end{array}$ & $\begin{array}{l}0.2709 \\
578.91 \\
1.4761\end{array}$ & $\begin{array}{l}0.2744 \\
584.84 \\
1.4798\end{array}$ & $\begin{array}{l}0.2779 \\
590.79 \\
1.4834\end{array}$ & $\begin{array}{l}0.2815 \\
596.75 \\
1.4871\end{array}$ & $\begin{array}{l}0.2850 \\
602.73 \\
1.4906\end{array}$ & $\begin{array}{l}0.2885 \\
608.71 \\
1.4942\end{array}$ \\
\hline 1550 & $\begin{array}{l}V \\
H \\
S\end{array}$ & $\begin{array}{l}0.2521 \\
561.04 \\
1.4632\end{array}$ & $\begin{array}{l}0.2555 \\
566.94 \\
1.4670\end{array}$ & $\begin{array}{l}0.2590 \\
572.86 \\
1.4708\end{array}$ & $\begin{array}{l}0.2624 \\
578.79 \\
1.4745\end{array}$ & $\begin{array}{l}0.2659 \\
584.73 \\
1.4782\end{array}$ & $\begin{array}{l}0.2693 \\
590.69 \\
1.4818\end{array}$ & $\begin{array}{l}0.2727 \\
596.65 \\
1.4855\end{array}$ & $\begin{array}{l}0.2761 \\
502.63 \\
1.4890\end{array}$ & $\begin{array}{l}0.2795 \\
608.62 \\
1.4926\end{array}$ \\
\hline 1600 & $\begin{array}{l}V \\
H \\
S\end{array}$ & $\begin{array}{l}0.2445 \\
560.91 \\
1.4616\end{array}$ & $\begin{array}{l}0.2478 \\
566.82 \\
1.4654\end{array}$ & $\begin{array}{l}0.2512 \\
572.74 \\
1.19692\end{array}$ & $\begin{array}{l}0.2545 \\
578.67 \\
1.4729\end{array}$ & $\begin{array}{l}0.2579 \\
584.62 \\
1.4766\end{array}$ & $\begin{array}{l}0.2612 \\
590.58 \\
1.4803\end{array}$ & $\begin{array}{l}0.2645 \\
596.55 \\
1.4839\end{array}$ & $\begin{array}{l}0.2678 \\
602.53 \\
1.4875\end{array}$ & $\begin{array}{l}0.2711 \\
608.53 \\
1.4910\end{array}$ \\
\hline 1650 & $\begin{array}{l}V \\
H \\
S\end{array}$ & $\begin{array}{l}0.2373 \\
560.77 \\
1.4601\end{array}$ & $\begin{array}{l}0.2406 \\
566.69 \\
1.4639\end{array}$ & $\begin{array}{l}0.2438 \\
572.62 \\
1.4677\end{array}$ & $\begin{array}{l}0.2471 \\
578.56 \\
1.4714\end{array}$ & $\begin{array}{l}0.2503 \\
584.51 \\
1.4751\end{array}$ & $\begin{array}{l}0.2536 \\
590.47 \\
1.4788\end{array}$ & $\begin{array}{l}0.2568 \\
596.45 \\
1.4824\end{array}$ & $\begin{array}{l}0.2600 \\
602.44 \\
1.4860\end{array}$ & $\begin{array}{l}0.2632 \\
608.43 \\
1.4895\end{array}$ \\
\hline 1700 & $\begin{array}{l}V \\
H \\
S\end{array}$ & $\begin{array}{l}0.2306 \\
560.64 \\
1.4586\end{array}$ & $\begin{array}{l}0.2337 \\
566.56 \\
1.4624\end{array}$ & $\begin{array}{l}0.2369 \\
572.50 \\
1.4662\end{array}$ & $\begin{array}{l}0.2401 \\
578.44 \\
1.4700\end{array}$ & $\begin{array}{l}0.2432 \\
584.40 \\
1.4737\end{array}$ & $\begin{array}{l}0.2464 \\
590.37 \\
1.4773\end{array}$ & $\begin{array}{l}0.2495 \\
596.35 \\
1.4809\end{array}$ & $\begin{array}{l}0.2527 \\
602.34 \\
1.4845\end{array}$ & $\begin{array}{l}0.2558 \\
608.34 \\
1.4881\end{array}$ \\
\hline 1750 & $\begin{array}{l}\mathrm{V} \\
\mathrm{H} \\
\mathrm{S}\end{array}$ & $\begin{array}{l}0.2242 \\
560.51 \\
1.4572\end{array}$ & $\begin{array}{l}0.2273 \\
566.44 \\
1.4610\end{array}$ & $\begin{array}{l}0.2304 \\
572.38 \\
1.4648\end{array}$ & $\begin{array}{l}0.2335 \\
578.33 \\
1.4685\end{array}$ & $\begin{array}{l}0.2365 \\
584.29 \\
1.4722\end{array}$ & $\begin{array}{l}0.2396 \\
590.26 \\
1.4759\end{array}$ & $\begin{array}{l}0.2427 \\
596.25 \\
1.4795\end{array}$ & $\begin{array}{l}0.2457 \\
602.24 \\
1.4831\end{array}$ & $\begin{array}{l}0.2438 \\
608.25 \\
1.4867\end{array}$ \\
\hline 1800 & $\begin{array}{l}\mathrm{V} \\
\mathrm{H} \\
\mathrm{S}\end{array}$ & $\begin{array}{l}0.2182 \\
560.38 \\
1.4558\end{array}$ & $\begin{array}{l}0.2212 \\
566.31 \\
1.4596\end{array}$ & $\begin{array}{l}0.2242 \\
572.25 \\
1.4634\end{array}$ & $\begin{array}{l}0.2272 \\
578.21 \\
1.4671\end{array}$ & $\begin{array}{l}0.2302 \\
584.18 \\
1.4708\end{array}$ & $\begin{array}{l}0.2332 \\
590.16 \\
1.4745\end{array}$ & $\begin{array}{l}0.2362 \\
596.15 \\
1.4781\end{array}$ & $\begin{array}{l}0.2392 \\
602.15 \\
1.4817\end{array}$ & $\begin{array}{l}0.2421 \\
608.16 \\
1.4853\end{array}$ \\
\hline 1850 & $\begin{array}{l}V \\
H \\
S\end{array}$ & $\begin{array}{l}0.2125 \\
560.24 \\
1.4544\end{array}$ & $\begin{array}{l}0.2155 \\
566.18 \\
1.4582\end{array}$ & $\begin{array}{l}0.2184 \\
572.13 \\
1.4620\end{array}$ & $\begin{array}{l}0.2213 \\
578.09 \\
1.4658\end{array}$ & $\begin{array}{l}0.2243 \\
584.07 \\
1.4695\end{array}$ & $\begin{array}{l}0.2272 \\
590.05 \\
1.4731\end{array}$ & $\begin{array}{l}0.2301 \\
596.04 \\
1.4768\end{array}$ & $\begin{array}{l}0.2330 \\
602.05 \\
1.4804\end{array}$ & $\begin{array}{l}0.2359 \\
608.07 \\
1.4839\end{array}$ \\
\hline 1900 & $\begin{array}{l}V \\
H \\
S\end{array}$ & $\begin{array}{l}0.2071 \\
560.11 \\
1.4531\end{array}$ & $\begin{array}{l}0.2100 \\
566.06 \\
1.4569\end{array}$ & $\begin{array}{l}0.2129 \\
572.01 \\
1.4607\end{array}$ & $\begin{array}{l}0.2157 \\
577.98 \\
1.4644\end{array}$ & $\begin{array}{l}0.2186 \\
583.95 \\
1.4681\end{array}$ & $\begin{array}{l}0.2214 \\
589.94 \\
1.4718\end{array}$ & $\begin{array}{l}0.2243 \\
595.94 \\
1.4755\end{array}$ & $\begin{array}{l}0.2271 \\
601.95 \\
1.4790\end{array}$ & $\begin{array}{l}0.2299 \\
607.97 \\
1.4826\end{array}$ \\
\hline 1950 & $\begin{array}{l}V \\
H \\
S\end{array}$ & $\begin{array}{l}0.2020 \\
559.98 \\
1.4518\end{array}$ & $\begin{array}{l}0.2048 \\
565.93 \\
1.4556\end{array}$ & $\begin{array}{l}0.2076 \\
571.89 \\
1.4594\end{array}$ & $\begin{array}{l}0.2104 \\
577.86 \\
1.4631\end{array}$ & $\begin{array}{l}0.2132 \\
583.84 \\
1.4669\end{array}$ & $\begin{array}{l}0.2160 \\
589.84 \\
1.4705\end{array}$ & $\begin{array}{l}0.2188 \\
595.84 \\
1.4742\end{array}$ & $\begin{array}{l}0.2215 \\
601.86 \\
1.4778\end{array}$ & $\begin{array}{l}0.2243 \\
607.88 \\
1.4813\end{array}$ \\
\hline 2000 & $\begin{array}{l}V \\
H \\
S\end{array}$ & $\begin{array}{l}0.1972 \\
559.85 \\
1.4505\end{array}$ & $\begin{array}{l}0.1999 \\
565.80 \\
1.4543\end{array}$ & $\begin{array}{l}0.2026 \\
571.77 \\
1.4581\end{array}$ & $\begin{array}{l}0.2054 \\
577.75 \\
1.4619\end{array}$ & $\begin{array}{l}0.2081 \\
583.73 \\
1.4656\end{array}$ & $\begin{array}{l}0.2108 \\
589.73 \\
1.4693\end{array}$ & $\begin{array}{l}0.2135 \\
595.74 \\
1.4729\end{array}$ & $\begin{array}{l}0.2162 \\
601.76 \\
1.4765\end{array}$ & $\begin{array}{l}0.2189 \\
607.79 \\
1.4801\end{array}$ \\
\hline
\end{tabular}




1320

29.5260

290

29.8579

634.93

640.83

26.2062

26.5111

1.7156

1.7189

1.7222

617.29

623.15

26.8160

27.1209

27.4257

634.93

1.7084

1.7118

1. 7151

640.83

1.7184

20.9679

617.28

21.2119
623.15

21.4558

629.03

21.6998

1.6984

1.7017

634.92
1.7051

21.9437

640.83

1.7084

17.4757
617.28

17.6790
623.14

17.3824

629.02

18.0857

634.01

18.2890

I. 6868

I. 6902

1.6936

1.6969

640.82
1.7002

15.5042
634.91

15.6785

640.82

617.27

623.14

629.01

1.6833

1.6866

I. 6900

1.6933

13.1104

617.26

13.2630

13.4155

12.5681

12.7207

629.01

634.90

640.81
1.6873

1.6773

1.6806

I. 6840

11.6553

617.26

11.7910

623.12

11.3266

679.000

12.0623

634.90
1.6787

12.1979

640.81
1.6820

10.4912
617.25

10.6133
623.12

10.7355

629.00

10.8576

634.89

10.9797
640.80

640.80

1.6739

1.6772

9.5388

9.6498

623.11

9.7609

9.8719

634.89

9.9829

640.80

1.6696

1.6729

8.7451

8.8469

1.6663

617.24

623.10

8.9487

9.0505

9.1523

634.88
1.6657

640.7

1.6690

1.6624

8.1675

8.0735

617.23

623.10
1.6554

8.2615

628.98

8.3555

634.87

8.4495

640.78

1.6654

1.6588

1.6621

1380

1400

1420

30.1897

646.75

30.5216

652.69

30.8535

658.64

1.7319 664.60

27.7306

28.3403 658.64

1. 7281

28.6451 664.60

1.7217

652.69

1.7249

22.6755

1.7313

22.1876

646.75

2.4316

652.68

22.6755
658.63

22.9194

1.7181

1.7213

18.492

646.74

18.6957

18.8900

19.1023

652.68

658.63

1.7009

664.59

I. 7131

15.8529

646.74

1.6965

16.201

16.3758

652.67

1.6998

658.6 ?

664.58

13.8732

14.0258

1.7030

1.7062

646.73

652.67

$14 \cdot 1793$

658.6

14.3309

1.6938

1.6970

664.58

1.7002

12.3335 646.73

12.6048

$\begin{array}{ll}658.61 & 564.058 \\ 1.6917 & 1.6949\end{array}$

11.1018 1.6885

646.72

11.2238 652.66

11.3459

658.61

11.4680

1.6805

1.6837

1.6870

1.6901

10.2050

10.3160

10.4270 652.66

658.61

664.57

1.6762

1.6795

1.6827

1.6859

$9.2541 \quad 9.3559$

9.4577

9.5594

646.71

652.65

658.60

664.57

8.5435

1.6819

646.71

8.6374

8.7314

8.8254

652.65

658.60

664.56

1.6687

1.6752

1.6783 


\begin{tabular}{|c|c|c|c|c|c|c|c|c|c|c|}
\hline \multicolumn{2}{|c|}{$\begin{array}{l}\text { PRESSURE } \\
\text { PSIA }\end{array}$} & 1260 & 1280 & 1300 & $\begin{array}{l}\text { TEMP } \\
1320\end{array}$ & $\begin{array}{r}\text { RATURE } \\
1340\end{array}$ & 1360 & 1380 & 1400 & 1420 \\
\hline 56 & $\begin{array}{l}V \\
H \\
S\end{array}$ & $\begin{array}{l}7.4979 \\
617.22 \\
1.6487\end{array}$ & $\begin{array}{l}7.5852 \\
673.09 \\
1.6521\end{array}$ & $\begin{array}{l}7.6725 \\
628.97 \\
1.6555\end{array}$ & $\begin{array}{l}7.7598 \\
634.87 \\
1.6588\end{array}$ & $\begin{array}{l}7.8471 \\
640.78 \\
1.6621\end{array}$ & $\begin{array}{l}7.9344 \\
646.70 \\
1.6654\end{array}$ & $\begin{array}{l}8.0216 \\
652.64 \\
1.6686\end{array}$ & $\begin{array}{l}8.1089 \\
658.59 \\
1.6718\end{array}$ & $\begin{array}{l}8.1962 \\
664.56 \\
1.6750\end{array}$ \\
\hline 60 & $\begin{array}{l}V \\
H \\
S\end{array}$ & $\begin{array}{l}6.9990 \\
617.22 \\
1.6456\end{array}$ & $\begin{array}{l}7.0805 \\
623.08 \\
1.6490\end{array}$ & $\begin{array}{l}7.1620 \\
628.97 \\
1.5524\end{array}$ & $\begin{array}{l}7.2435 \\
634.86 \\
1.6557\end{array}$ & $\begin{array}{l}7.3250 \\
640.77 \\
1.6590\end{array}$ & $\begin{array}{l}7.4065 \\
646.70 \\
1.6523\end{array}$ & $\begin{array}{l}7.4879 \\
652.64 \\
1.6655\end{array}$ & $\begin{array}{l}7.5694 \\
658.59 \\
1.6687\end{array}$ & $\begin{array}{l}7.6509 \\
664.55 \\
1.6719\end{array}$ \\
\hline 70 & $\begin{array}{l}V \\
H \\
S\end{array}$ & $\begin{array}{l}6.0012 \\
617.20 \\
1.6387\end{array}$ & $\begin{array}{l}6.0711 \\
623.07 \\
1.6421\end{array}$ & $\begin{array}{l}6.1410 \\
623.95 \\
1.6454\end{array}$ & $\begin{array}{l}6.2109 \\
634.85 \\
1.6488\end{array}$ & $\begin{array}{l}6.2808 \\
640.76 \\
1.6521\end{array}$ & $\begin{array}{l}6.3506 \\
646.69 \\
1.6553\end{array}$ & $\begin{array}{l}6.4205 \\
652.63 \\
1.6586\end{array}$ & $\begin{array}{l}6.4904 \\
658.58 \\
1.6618\end{array}$ & $\begin{array}{l}6.5602 \\
664.54 \\
1.6650\end{array}$ \\
\hline 80 & $\begin{array}{l}V \\
H \\
S\end{array}$ & $\begin{array}{l}5.2529 \\
617.18 \\
1.6327\end{array}$ & $\begin{array}{l}5.3141 \\
623.05 \\
1.6360\end{array}$ & $\begin{array}{l}5.3753 \\
628.94 \\
1.6394\end{array}$ & $\begin{array}{l}5.4364 \\
634.83 \\
1.6427\end{array}$ & $\begin{array}{l}5.4976 \\
640.75 \\
1.6460\end{array}$ & $\begin{array}{l}5.5588 \\
646.67 \\
1.6493\end{array}$ & $\begin{array}{l}5.6200 \\
652.61 \\
1.6526\end{array}$ & $\begin{array}{l}5.6811 \\
658.57 \\
1.5558\end{array}$ & $\begin{array}{l}5.7423 \\
664.53 \\
1.6590\end{array}$ \\
\hline 90 & $\begin{array}{l}V \\
H \\
S\end{array}$ & $\begin{array}{l}4.6708 \\
617.16 \\
1.6273\end{array}$ & $\begin{array}{l}4.7252 \\
623.03 \\
1.6307\end{array}$ & $\begin{array}{l}4.7797 \\
623.92 \\
1.5341\end{array}$ & $\begin{array}{l}4.8341 \\
634.32 \\
1.6374\end{array}$ & $\begin{array}{l}4.8885 \\
540.72 \\
1.6407\end{array}$ & $\begin{array}{l}4.9429 \\
646.66 \\
1.6440\end{array}$ & $\begin{array}{l}4.9973 \\
652.60 \\
1.6472\end{array}$ & $\begin{array}{l}5.0517 \\
658.56 \\
1.6505\end{array}$ & $\begin{array}{l}5.1061 \\
664: 53 \\
1.6537\end{array}$ \\
\hline 100 & $\begin{array}{l}V \\
H \\
S\end{array}$ & $\begin{array}{l}4.2052 \\
617.15 \\
1.6226\end{array}$ & $\begin{array}{l}4.2542 \\
623.02 \\
1.6260\end{array}$ & $\begin{array}{l}4.3032 \\
628.91 \\
1.6293\end{array}$ & $\begin{array}{l}4.3522 \\
634.81 \\
1.6327\end{array}$ & $\begin{array}{l}4.4012 \\
640.72 \\
1.6360\end{array}$ & $\begin{array}{l}4.4502 \\
646.65 \\
1.6393\end{array}$ & $\begin{array}{l}4.4992 \\
652.59 \\
1.6425\end{array}$ & $\begin{array}{l}4.5481 \\
653.55 \\
1.6457\end{array}$ & $\begin{array}{l}4.5971 \\
662.52 \\
1.6489\end{array}$ \\
\hline 110 & $\begin{array}{l}V \\
H \\
S\end{array}$ & $\begin{array}{l}3.8242 \\
617.13 \\
1.6183\end{array}$ & $\begin{array}{l}3.8688 \\
623.00 \\
1.6217\end{array}$ & $\begin{array}{l}3.7134 \\
628.89 \\
1.6250\end{array}$ & $\begin{array}{l}3.0579 \\
634.79 \\
1.6284\end{array}$ & $\begin{array}{l}4.0025 \\
640.71 \\
1.6317\end{array}$ & $\begin{array}{l}4.0471 \\
546.64 \\
1.6350\end{array}$ & $\begin{array}{l}4.0916 \\
652.58 \\
1.6382\end{array}$ & $\begin{array}{l}4.1361 \\
653054 \\
1.6414\end{array}$ & $\begin{array}{l}4.1807 \\
664051 \\
1.6446\end{array}$ \\
\hline 120 & $\begin{array}{l}V \\
H \\
S\end{array}$ & $\begin{array}{l}3.5067 \\
617.11 \\
1.6144\end{array}$ & $\begin{array}{l}3.5476 \\
622.99 \\
1.6178\end{array}$ & $\begin{array}{l}3.5885 \\
628.87 \\
1.5211\end{array}$ & $\begin{array}{l}3.6294 \\
634.78 \\
1.6245\end{array}$ & $\begin{array}{l}3.6702 \\
640.69 \\
1.6278\end{array}$ & $\begin{array}{l}3.7111 \\
646.63 \\
1.6310\end{array}$ & $\begin{array}{l}3.7520 \\
652.57 \\
1.6343\end{array}$ & $\begin{array}{l}3.7928 \\
658.53 \\
1.6375\end{array}$ & $\begin{array}{l}3.8337 \\
564550 \\
1.6407\end{array}$ \\
\hline 130 & $\begin{array}{l}V \\
H \\
S\end{array}$ & $\begin{array}{l}3.2381 \\
617.09 \\
1.6107\end{array}$ & $\begin{array}{l}3.2758 \\
622.97 \\
1.6141\end{array}$ & $\begin{array}{l}3.3136 \\
628.86 \\
1.6175\end{array}$ & $\begin{array}{l}3.3514 \\
634.76 \\
1.6208\end{array}$ & $\begin{array}{l}3.3891 \\
640.68 \\
1.6241\end{array}$ & $\begin{array}{l}3.4268 \\
646.61 \\
1.6274\end{array}$ & $\begin{array}{l}3.4646 \\
652.56 \\
1.6307\end{array}$ & $\begin{array}{l}3.5023 \\
658.52 \\
1.6339\end{array}$ & $\begin{array}{l}3.5400 \\
664049 \\
1.6371\end{array}$ \\
\hline 140 & $\begin{array}{l}V \\
H \\
S\end{array}$ & $\begin{array}{l}3.0078 \\
617.08 \\
1.6074\end{array}$ & $\begin{array}{l}3.0429 \\
622.95 \\
1.6108\end{array}$ & $\begin{array}{l}3.0780 \\
628.84 \\
1.6142\end{array}$ & $\begin{array}{l}3.1131 \\
634.75 \\
1.6175\end{array}$ & $\begin{array}{l}3.1481 \\
640.67 \\
1.6208\end{array}$ & $\begin{array}{l}3.1832 \\
646.60 \\
1.6241\end{array}$ & $\begin{array}{l}3.2182 \\
652.55 \\
1.6273\end{array}$ & $\begin{array}{l}3.2533 \\
658.51 \\
1.6305\end{array}$ & $\begin{array}{l}3.2883 \\
664.48 \\
1.6337\end{array}$ \\
\hline 150 & $\begin{array}{l}V \\
H \\
S\end{array}$ & $\begin{array}{l}2.8082 \\
617.06 \\
1.6043\end{array}$ & $\begin{array}{l}2.8410 \\
622.944 \\
1.6077\end{array}$ & $\begin{array}{l}2.8738 \\
628.83 \\
1.5110\end{array}$ & $\begin{array}{l}2.9065 \\
634.74 \\
1.6144\end{array}$ & $\begin{array}{l}2.9393 \\
540.66 \\
1.6177\end{array}$ & $\begin{array}{l}2.9720 \\
646.59 \\
1.6210\end{array}$ & $\begin{array}{l}3.0048 \\
652.54 \\
1.6242\end{array}$ & $\begin{array}{l}3.0375 \\
658.50 \\
1.6274\end{array}$ & $\begin{array}{l}3.0702 \\
66<.47 \\
1.6306\end{array}$ \\
\hline
\end{tabular}




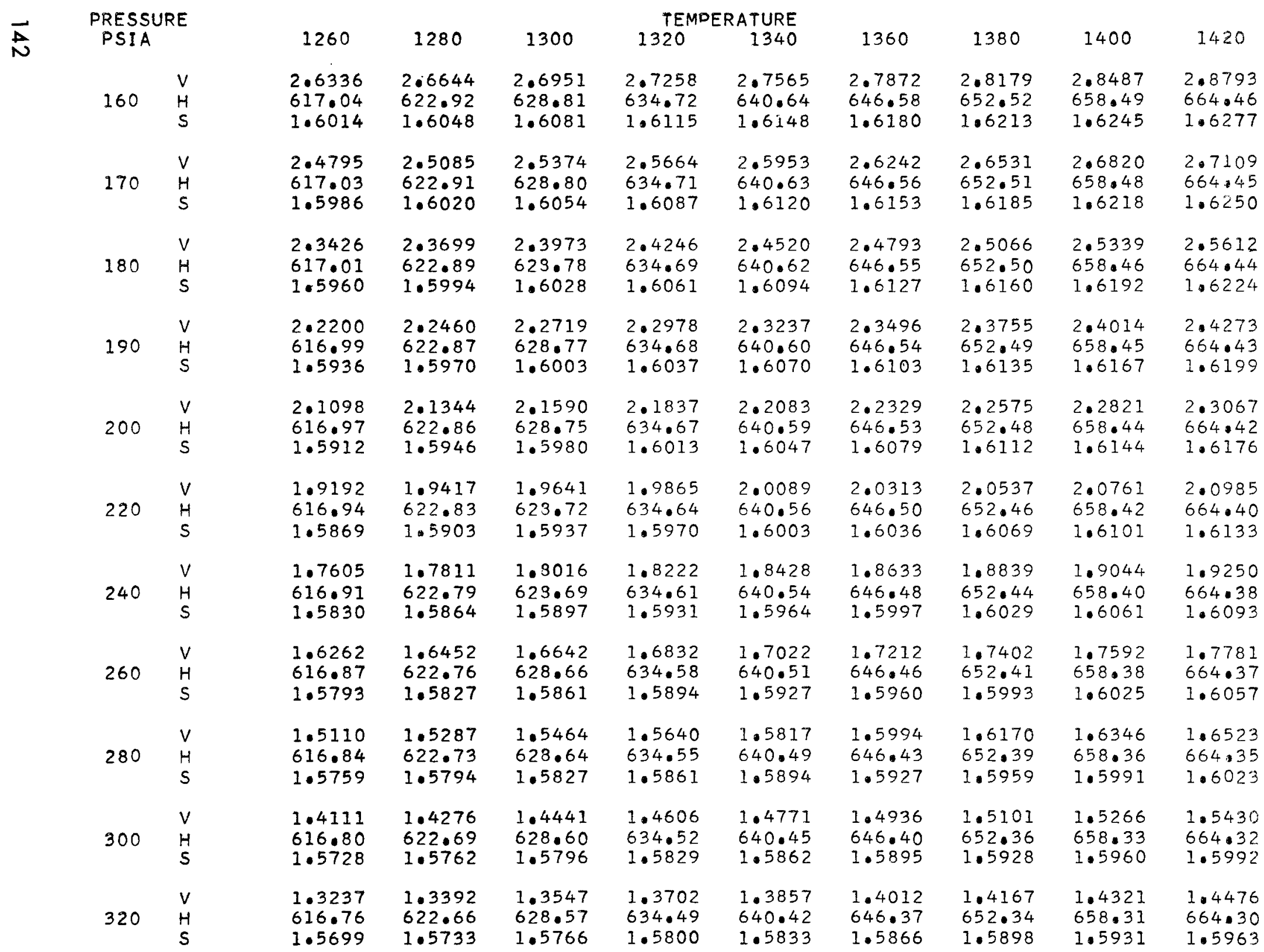


PRESSURE PSIA

$\begin{array}{ll} & V \\ 340 & H \\ & S\end{array}$

$\begin{array}{ll} & V \\ 360 & H \\ & S\end{array}$

380

400

$420 \quad \begin{array}{r}\mathrm{H} \\ \\ \end{array}$

440

460

480

$500 \quad \frac{V}{S}$

520

$\begin{array}{lll}\vec{\omega} & 540 \quad \mathrm{H} \\ \mathrm{\omega} & & \mathrm{S}\end{array}$

\begin{tabular}{|c|c|c|c|c|c|c|c|c|}
\hline \multicolumn{9}{|c|}{ TEMPERATURE } \\
\hline 1260 & 1280 & 1300 & 1320 & 1340 & 1360 & 1380 & 1400 & 1420 \\
\hline $\begin{array}{l}1.2467 \\
616.73 \\
1.5671\end{array}$ & $\begin{array}{l}1.2613 \\
622.62 \\
1.5705\end{array}$ & $\begin{array}{l}1.2759 \\
628.54 \\
1.5739\end{array}$ & $\begin{array}{l}1.2905 \\
634.46 \\
1.5772\end{array}$ & $\begin{array}{l}1.3051 \\
640.40 \\
1.5805\end{array}$ & $\begin{array}{l}1.3196 \\
646.35 \\
1.5838\end{array}$ & $\begin{array}{l}1.3342 \\
652.31 \\
1.5871\end{array}$ & $\begin{array}{l}1.3488 \\
658.29 \\
1.5903\end{array}$ & $\begin{array}{l}1.3634 \\
664 \cdot 28 \\
1.5935\end{array}$ \\
\hline $\begin{array}{l}1.1782 \\
616.69 \\
1.5645\end{array}$ & $\begin{array}{l}1.1920 \\
622.59 \\
1.5679\end{array}$ & $\begin{array}{l}1.2058 \\
628.50 \\
1.5713\end{array}$ & $\begin{array}{l}1.2196 \\
634.43 \\
1.5746\end{array}$ & $\begin{array}{l}1.2334 \\
640.37 \\
1.5779\end{array}$ & $\begin{array}{l}1.2472 \\
646.32 \\
1.5812\end{array}$ & $\begin{array}{l}1.2609 \\
652.29 \\
1.5845\end{array}$ & $\begin{array}{l}1.2747 \\
658.27 \\
1.5877\end{array}$ & $\begin{array}{l}1.2885 \\
664.26 \\
1.5909\end{array}$ \\
\hline $\begin{array}{l}1.1169 \\
616.66 \\
1.5620\end{array}$ & $\begin{array}{l}1.1300 \\
622.56 \\
1.5654\end{array}$ & $\begin{array}{l}1.1431 \\
628.47 \\
1.5688\end{array}$ & $\begin{array}{l}1.1561 \\
634.40 \\
1.5721\end{array}$ & $\begin{array}{l}1.1692 \\
640.34 \\
1.5754\end{array}$ & $\begin{array}{l}1.1823 \\
646.30 \\
1.5787\end{array}$ & $\begin{array}{l}1.1954 \\
652.27 \\
1.5820\end{array}$ & $\begin{array}{l}1.2084 \\
658.25 \\
1.5852\end{array}$ & $\begin{array}{l}1.2215 \\
664.24 \\
1.5884\end{array}$ \\
\hline $\begin{array}{l}1.0617 \\
616.62 \\
1.5596\end{array}$ & $\begin{array}{l}1.0742 \\
622.53 \\
1.5631\end{array}$ & $\begin{array}{l}1.0866 \\
628.44 \\
1.5664\end{array}$ & $\begin{array}{l}1.0991 \\
634.37 \\
1.5698\end{array}$ & $\begin{array}{l}1.1115 \\
640.32 \\
1.5731\end{array}$ & $\begin{array}{l}1.1239 \\
646.27 \\
1.5764\end{array}$ & $\begin{array}{l}1.1363 \\
652.24 \\
1.5796\end{array}$ & $\begin{array}{l}1.1488 \\
658.22 \\
1.5829\end{array}$ & $\begin{array}{l}1.1612 \\
664.22 \\
1.5861\end{array}$ \\
\hline $\begin{array}{l}1.0118 \\
616.59 \\
1.5574\end{array}$ & $\begin{array}{l}1.0237 \\
622.49 \\
1.5608\end{array}$ & $\begin{array}{l}1.0355 \\
628.41 \\
1.5642\end{array}$ & $\begin{array}{l}1.0474 \\
634.34 \\
1.5675\end{array}$ & $\begin{array}{l}1.0592 \\
640.29 \\
1.5709\end{array}$ & $\begin{array}{l}1.0711 \\
646.25 \\
1.5742\end{array}$ & $\begin{array}{l}1.0829 \\
652.22 \\
1.5774\end{array}$ & $\begin{array}{l}1.0948 \\
658.20 \\
1.5806\end{array}$ & $\begin{array}{l}1.1066 \\
664.20 \\
1.5839\end{array}$ \\
\hline $\begin{array}{l}0.9664 \\
616.55 \\
1.5553\end{array}$ & $\begin{array}{l}0.9778 \\
622.46 \\
1.5587\end{array}$ & $\begin{array}{l}0.9891 \\
623.38 \\
1.5621\end{array}$ & $\begin{array}{l}1.0004 \\
634.32 \\
1.5654\end{array}$ & $\begin{array}{l}1.0118 \\
640.26 \\
1.5687\end{array}$ & $\begin{array}{l}1.0231 \\
646.22 \\
1.5720\end{array}$ & $\begin{array}{l}1.0344 \\
652.20 \\
1.5753\end{array}$ & $\begin{array}{l}1.0457 \\
658.18 \\
1.5785\end{array}$ & $\begin{array}{l}1.0570 \\
664.18 \\
1.5817\end{array}$ \\
\hline $\begin{array}{l}0.9250 \\
616.52 \\
1.5532\end{array}$ & $\begin{array}{l}0.9358 \\
622.43 \\
1.5566\end{array}$ & $\begin{array}{l}0.9467 \\
628.35 \\
1.5600\end{array}$ & $\begin{array}{l}0.9575 \\
634.29 \\
1.5634\end{array}$ & $\begin{array}{l}0.9684 \\
640.24 \\
1.5667\end{array}$ & $\begin{array}{l}0.9792 \\
646.20 \\
1.5700\end{array}$ & $\begin{array}{l}0.9901 \\
652.17 \\
1.5732\end{array}$ & $\begin{array}{l}1.0009 \\
658.16 \\
1.5765\end{array}$ & $\begin{array}{l}1.0117 \\
664.16 \\
1.5797\end{array}$ \\
\hline $\begin{array}{l}0.8870 \\
616.48 \\
1.5513\end{array}$ & $\begin{array}{l}0.8974 \\
622.40 \\
1.5547\end{array}$ & $\begin{array}{l}0.9078 \\
628.32 \\
1.5581\end{array}$ & $\begin{array}{l}0.9182 \\
634.26 \\
1.5614\end{array}$ & $\begin{array}{l}0.9286 \\
640.21 \\
1.5647\end{array}$ & $\begin{array}{l}0.9390 \\
646.17 \\
1.5680\end{array}$ & $\begin{array}{l}0.9494 \\
652.15 \\
1.5713\end{array}$ & $\begin{array}{l}0.9598 \\
658.14 \\
1.5745\end{array}$ & $\begin{array}{l}0.9702 \\
664.14 \\
1.5777\end{array}$ \\
\hline $\begin{array}{l}0.8520 \\
616.45 \\
1.5494\end{array}$ & $\begin{array}{l}0.8621 \\
622.36 \\
1.5528\end{array}$ & $\begin{array}{l}0.8721 \\
628.29 \\
1.5562\end{array}$ & $\begin{array}{l}0.8821 \\
634.23 \\
1.5595\end{array}$ & $\begin{array}{l}0.8921 \\
640.18 \\
1.5629\end{array}$ & $\begin{array}{l}0.9021 \\
646.15 \\
1.5662\end{array}$ & $\begin{array}{l}0.9120 \\
652.13 \\
1.5694\end{array}$ & $\begin{array}{l}0.9220 \\
658.12 \\
1.5727\end{array}$ & $\begin{array}{l}0.9320 \\
664.12 \\
1.5759\end{array}$ \\
\hline $\begin{array}{l}0.8198 \\
616.41 \\
1.5476\end{array}$ & $\begin{array}{l}0.8294 \\
622.33 \\
1.5510\end{array}$ & $\begin{array}{l}0.3390 \\
628.26 \\
1.5544\end{array}$ & $\begin{array}{l}0.8487 \\
634.20 \\
1.5577\end{array}$ & $\begin{array}{l}0.8583 \\
640.15 \\
1.5611\end{array}$ & $\begin{array}{l}0.8679 \\
646.12 \\
1.5644\end{array}$ & $\begin{array}{l}0.8775 \\
652.10 \\
1.5676\end{array}$ & $\begin{array}{l}0.8871 \\
658.09 \\
1.5709\end{array}$ & $\begin{array}{l}0.8967 \\
664.10 \\
1.5741\end{array}$ \\
\hline $\begin{array}{l}0.7899 \\
616.38 \\
1.5458\end{array}$ & $\begin{array}{l}0.7992 \\
622.30 \\
1.5492\end{array}$ & $\begin{array}{l}0.8085 \\
628.23 \\
1.5526\end{array}$ & $\begin{array}{l}0.8178 \\
634.17 \\
1.5560\end{array}$ & $\begin{array}{l}0.8270 \\
640.13 \\
1.5593\end{array}$ & $\begin{array}{l}0.8363 \\
646.10 \\
1.5626\end{array}$ & $\begin{array}{l}0.8456 \\
652.08 \\
1.5659\end{array}$ & $\begin{array}{l}0.8548 \\
658.07 \\
1.5691\end{array}$ & $\begin{array}{l}0.8641 \\
664.08 \\
1.5723\end{array}$ \\
\hline
\end{tabular}




\begin{tabular}{|c|c|c|c|c|c|c|c|c|c|c|}
\hline 560 & $\begin{array}{l}V \\
H \\
S\end{array}$ & $\begin{array}{l}0.7622 \\
616.34 \\
1.5441\end{array}$ & $\begin{array}{l}0.7711 \\
622.26 \\
1.5476\end{array}$ & $\begin{array}{l}0.7801 \\
628.20 \\
1.5510\end{array}$ & $\begin{array}{l}0.7890 \\
634.14 \\
1.5543\end{array}$ & $\begin{array}{l}0.7980 \\
640.10 \\
1.5576\end{array}$ & $\begin{array}{l}0.8069 \\
646.07 \\
1.5609\end{array}$ & $\begin{array}{l}0.8159 \\
652.05 \\
1.5642\end{array}$ & $\begin{array}{l}0.8248 \\
658.05 \\
1.5674\end{array}$ & $\begin{array}{l}0.8338 \\
664.06 \\
1.5707\end{array}$ \\
\hline 580 & $\begin{array}{l}V \\
H \\
S\end{array}$ & $\begin{array}{l}0.7363 \\
616.31 \\
1.5425\end{array}$ & $\begin{array}{l}0.7450 \\
622.23 \\
1.5459\end{array}$ & $\begin{array}{l}0.7537 \\
628.17 \\
1.5493\end{array}$ & $\begin{array}{l}0.7623 \\
634.11 \\
1.5527\end{array}$ & $\begin{array}{l}0.7710 \\
640.07 \\
1.5560\end{array}$ & $\begin{array}{l}0.7796 \\
646.05 \\
1.5593\end{array}$ & $\begin{array}{l}0.7883 \\
652.03 \\
1.5626\end{array}$ & $\begin{array}{l}0.7969 \\
658.03 \\
1.5658\end{array}$ & $\begin{array}{l}0.8055 \\
664.04 \\
1.5690\end{array}$ \\
\hline 600 & $\begin{array}{l}V \\
H \\
S\end{array}$ & $\begin{array}{l}0.7122 \\
616.28 \\
1.5410\end{array}$ & $\begin{array}{l}0.7206 \\
622.20 \\
1.5444\end{array}$ & $\begin{array}{l}0.7290 \\
628.13 \\
1.5478\end{array}$ & $\begin{array}{l}0.7374 \\
634.08 \\
1.5511\end{array}$ & $\begin{array}{l}0.7457 \\
640.05 \\
1.5545\end{array}$ & $\begin{array}{l}0.7541 \\
646.02 \\
1.5578\end{array}$ & $\begin{array}{l}0.7625 \\
652.01 \\
1.5610\end{array}$ & $\begin{array}{l}0.7708 \\
658.01 \\
1.5643\end{array}$ & $\begin{array}{l}0.7792 \\
664.02 \\
1.5675\end{array}$ \\
\hline 620 & $\begin{array}{l}V \\
H \\
S\end{array}$ & $\begin{array}{l}0.6897 \\
616.24 \\
1.5394\end{array}$ & $\begin{array}{l}0.6978 \\
622.17 \\
1.5429\end{array}$ & $\begin{array}{l}0.7059 \\
628.10 \\
1.5462\end{array}$ & $\begin{array}{l}0.7140 \\
634.05 \\
1.5496\end{array}$ & $\begin{array}{l}0.7221 \\
640.02 \\
1.5529\end{array}$ & $\begin{array}{l}0.7302 \\
645.99 \\
1.5562\end{array}$ & $\begin{array}{l}0.7383 \\
651.98 \\
1.5595\end{array}$ & $\begin{array}{l}0.7464 \\
657.98 \\
1.5628\end{array}$ & $\begin{array}{l}0.7545 \\
654.00 \\
1.5660\end{array}$ \\
\hline 640 & $\begin{array}{l}V \\
H \\
S\end{array}$ & $\begin{array}{l}0.6685 \\
616.21 \\
1.5380\end{array}$ & $\begin{array}{l}0.6764 \\
622.13 \\
1.5414\end{array}$ & $\begin{array}{l}0.6843 \\
628.07 \\
1.5448\end{array}$ & $\begin{array}{l}0.6921 \\
634.03 \\
1.5481\end{array}$ & $\begin{array}{l}0.7000 \\
639.99 \\
1.5515\end{array}$ & $\begin{array}{l}0.7079 \\
645.97 \\
1.5548\end{array}$ & $\begin{array}{l}0.7157 \\
651.96 \\
1.5580\end{array}$ & $\begin{array}{l}0.7236 \\
657.96 \\
1.5613\end{array}$ & $\begin{array}{l}0.7314 \\
663.98 \\
1.5645\end{array}$ \\
\hline 660 & $\begin{array}{l}V \\
H \\
S\end{array}$ & $\begin{array}{l}0.6486 \\
616.17 \\
1.5365\end{array}$ & $\begin{array}{l}0.6563 \\
622.10 \\
1.5400\end{array}$ & $\begin{array}{l}0.6639 \\
623.04 \\
1.5433\end{array}$ & $\begin{array}{l}0.5716 \\
634.00 \\
1.5467\end{array}$ & $\begin{array}{l}0.6792 \\
639.96 \\
1.5500\end{array}$ & $\begin{array}{l}0.6868 \\
645.94 \\
1.5533\end{array}$ & $\begin{array}{l}0.6945 \\
651.94 \\
1.5566\end{array}$ & $\begin{array}{l}0.7021 \\
657.94 \\
1.5599\end{array}$ & $\begin{array}{l}0.7097 \\
663.96 \\
1.5631\end{array}$ \\
\hline 680 & $\begin{array}{l}V \\
H \\
S\end{array}$ & $\begin{array}{l}0.6299 \\
616.14 \\
1.5351\end{array}$ & $\begin{array}{l}0.6374 \\
622.07 \\
1.5386\end{array}$ & $\begin{array}{l}0.5448 \\
628.01 \\
1.5420\end{array}$ & $\begin{array}{l}0.6522 \\
633.97 \\
1.5453\end{array}$ & $\begin{array}{l}0.6596 \\
639.94 \\
1.5487\end{array}$ & $\begin{array}{l}0.6670 \\
645.92 \\
1.5520\end{array}$ & $\begin{array}{l}0.6744 \\
651.91 \\
1.5552\end{array}$ & $\begin{array}{l}0.6818 \\
657.92 \\
1.5585\end{array}$ & $\begin{array}{l}0.6892 \\
663.94 \\
1.5617\end{array}$ \\
\hline 700 & $\begin{array}{l}V \\
H \\
S\end{array}$ & $\begin{array}{l}0.6123 \\
616.10 \\
1.5338\end{array}$ & $\begin{array}{l}0.6195 \\
622.03 \\
1.5372\end{array}$ & $\begin{array}{l}0.6268 \\
627.98 \\
1.5406\end{array}$ & $\begin{array}{l}0.6340 \\
633.94 \\
1.5440\end{array}$ & $\begin{array}{l}0.6412 \\
639.91 \\
1.5473\end{array}$ & $\begin{array}{l}0.6484 \\
645.89 \\
1.5506\end{array}$ & $\begin{array}{l}0.6556 \\
651.89 \\
1.5539\end{array}$ & $\begin{array}{l}0.6628 \\
657.90 \\
1.5571\end{array}$ & $\begin{array}{l}0.6700 \\
663.92 \\
1.5604\end{array}$ \\
\hline 720 & $\begin{array}{l}V \\
H \\
S\end{array}$ & $\begin{array}{l}0.5956 \\
616.07 \\
1.5325\end{array}$ & $\begin{array}{l}0.6027 \\
622.00 \\
1.5359\end{array}$ & $\begin{array}{l}0.6097 \\
627.95 \\
1.5393\end{array}$ & $\begin{array}{l}0.6167 \\
633.91 \\
1.5427\end{array}$ & $\begin{array}{l}0.6237 \\
639.88 \\
1.5460\end{array}$ & $\begin{array}{l}0.6308 \\
645.87 \\
1.5493\end{array}$ & $\begin{array}{l}0.6378 \\
651.86 \\
1.5526\end{array}$ & $\begin{array}{l}0.6448 \\
657.87 \\
1.5558\end{array}$ & $\begin{array}{l}0.6518 \\
663.90 \\
1.5590\end{array}$ \\
\hline 740 & $\begin{array}{l}V \\
H \\
S\end{array}$ & $\begin{array}{l}0.5799 \\
616.03 \\
1.5312\end{array}$ & $\begin{array}{l}0.5867 \\
621.97 \\
1.5346\end{array}$ & $\begin{array}{l}0.5936 \\
627.92 \\
1.5380\end{array}$ & $\begin{array}{l}0.6004 \\
633.88 \\
1.5414\end{array}$ & $\begin{array}{l}0.6073 \\
639.85 \\
1.5447\end{array}$ & $\begin{array}{l}0.6141 \\
645.84 \\
1.5480\end{array}$ & $\begin{array}{l}0.6209 \\
651.84 \\
1.5513\end{array}$ & $\begin{array}{l}0.6277 \\
657.85 \\
1.5546\end{array}$ & $\begin{array}{l}0.6345 \\
663.88 \\
1.5578\end{array}$ \\
\hline 760 & $\begin{array}{l}V \\
H \\
S\end{array}$ & $\begin{array}{l}0.5650 \\
616.00 \\
1.5300\end{array}$ & $\begin{array}{l}0.5716 \\
621.94 \\
1.5334\end{array}$ & $\begin{array}{l}0.5783 \\
627.89 \\
1.5368\end{array}$ & $\begin{array}{l}0.5850 \\
633.85 \\
1.5402\end{array}$ & $\begin{array}{l}0.5916 \\
639.83 \\
1.5435\end{array}$ & $\begin{array}{l}0.5983 \\
645.82 \\
1.5468\end{array}$ & $\begin{array}{l}0.6049 \\
651.82 \\
1.5501\end{array}$ & $\begin{array}{l}0.6116 \\
657.83 \\
1.5533\end{array}$ & $\begin{array}{l}0.6182 \\
663.85 \\
1.5565\end{array}$ \\
\hline
\end{tabular}




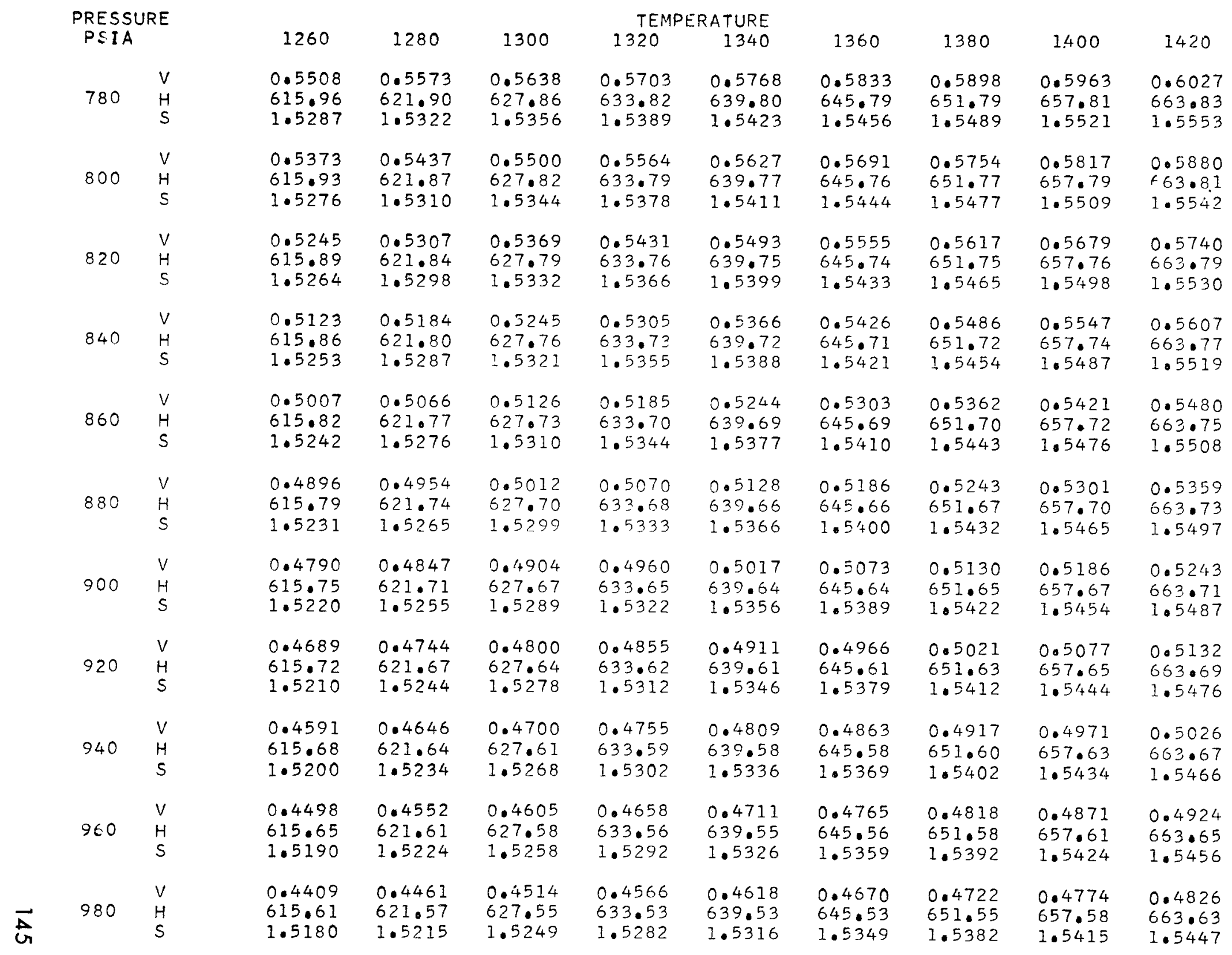


1000

$0.4323 \quad 0.4374$

1000

0.14426

1320

1340

1360

1380

1400

1420

\subsection{1}

1.5205

627.51

0.4477

0.4528

$633.50 \quad 639.50$

$1.5273 \quad 1.5306$

0.4579

0.4630

0.4681

0.4732

0.4291

0.4241

615.54

621.51

0.4341

627.48

0.4392

633.47

0.4442

1.5264

1.5297

1.5340

1.5372

657.56

663.61

1.5437

\section{$0.4161 \quad 0.4211$}

$615.51-621.47$

$1.5152 \quad 1.5186$

0.4260

627.45

0.4310

633.44

0.4359

639.44

0.4492

645.48

0.4542

651.50

0.4592

0.4642

1.5330

1.5363

1.5396

663.58

0.4408

0.4457

651.48

0.4507

1.5428

$0.4085 \quad 0.4134$

615047

621.44

0.4182

1.5254

1.5288

645.46
1.5321

1.5354

657.52

0.4556

663.56

0.42310 .4279

633.41

639.41

0.4328

0.4376

1.5387

1.5419

1.5143

627.42

1. 5245

1.5279

645.43

651.46

0.4424

0.4472

1.5312

1.5345

657.49

663.54

0.40120 .4059

0.4107

0.4155

0.4202

0.4250

0.4297

1.5378

1.5410

1.5134

621.41
1.5169

627.39
1.5203

633.28
1.5237

0.39
1.5270

645.40

651.43

0.4345

0.4392

1.5303

1.5336

657.47

$0.3941 \quad 0.3988$

0.4035

0.4081

627.36

633.35

0.4128

539.36

0.4175

645.38

0.4221

651.41

1.5369

1.5401

1.51251 .5160

]. 5228

1.5261

1.5295

1.5328

0.4268

657.45

0.4315

663.50

$\begin{array}{lll}0.3873 & 0.3919 & 0.3965 \\ 615.37 & 621.34 & 627.33\end{array}$

$0.4011 \quad 0.4057$

633.32

639.33

0.4103

0.4148

I. 5360

1.5393

$1.5117 \quad 1.5151$

1.5186

1.5219

1.5253

645.35
1.5286

651.38

0.4194

0.4240

1.5319

1.5352

663.48

$0.3943 \quad 0.3988$

0.4033

0.4078

0.4123

1.5384

$\begin{array}{lllll}615.33 & 621.31 & 627.30 & 633.29 & 639.30\end{array}$

645.33

651.36

657.40

0.4168

1.5278

1.5311

1.5343

663.46

$0.3743 \quad 0.3788$

0.3832

0.3877

0.3921

0.3966

0.4010

0.4054

1.5376

$615.30 \quad 621.28$

627.26

633.26

639.28

645.30

651.33

657.38

0.4098

$1.5203 \quad 1.5236$

1.5270

1.5303

1.5335

663.44

$\begin{array}{lllll}0.3682 & 0.3726 & 0.3769 & 0.3813 & 0.3857\end{array}$

$\begin{array}{ll}0.35 .27 & 621.24 \\ 1.5092 & 1.5127\end{array}$

0.3769
627.23
1.5161

633.24
1.5195

0.3901

639.25
1.5228

0.3901
645.27

0.3944

0.3988

1.5161

1.5261

651.31

657.36

1.5368

$0.3622 \quad 0.3665$

615.23

621.21

0.3709

0.3752

0.3795

0.3838

1.5294

1.5377

0.4031

663.41

1.5354

$633.21 \quad 639.22$

645.25

0.3881

0.3923

0.3966

$\begin{array}{lll}1.5153 & 1.5187 & 1.5220\end{array}$

1.5253

1.5286

657.33

663.39

1.5352 


\begin{tabular}{|c|c|c|c|c|c|c|c|c|c|c|}
\hline \multirow{2}{*}{\multicolumn{2}{|c|}{$\begin{array}{l}\text { PRESSURE } \\
\text { PSIA }\end{array}$}} & \multicolumn{8}{|c|}{ TEMPERATURE } & \multirow[b]{2}{*}{1420} \\
\hline & & 1260 & 1280 & 1300 & 1320 & & 1360 & 1380 & 1400 & \\
\hline 1220 & $\begin{array}{l}V \\
H \\
S\end{array}$ & $\begin{array}{l}0.3565 \\
615.20 \\
1.5076\end{array}$ & $\begin{array}{l}0.3607 \\
621.18 \\
1.5111\end{array}$ & $\begin{array}{l}0.3650 \\
627.17 \\
1.5145\end{array}$ & $\begin{array}{l}0.3692 \\
633.18 \\
1.5179\end{array}$ & $\begin{array}{l}0.3734 \\
639.19 \\
1.5212\end{array}$ & $\begin{array}{l}0.3777 \\
645.22 \\
1.5246\end{array}$ & $\begin{array}{l}0.3819 \\
651.26 \\
1.5279\end{array}$ & $\begin{array}{l}0.3861 \\
657.31 \\
1.5311\end{array}$ & $\begin{array}{l}0.3903 \\
663.37 \\
1.5344\end{array}$ \\
\hline 1240 & $\begin{array}{l}V \\
H \\
S\end{array}$ & $\begin{array}{l}0.3509 \\
615.16 \\
1.5068\end{array}$ & $\begin{array}{l}0.3551 \\
621.14 \\
1.5103\end{array}$ & $\begin{array}{l}0.3593 \\
627.14 \\
1.5137\end{array}$ & $\begin{array}{l}0.3635 \\
633.15 \\
1.5171\end{array}$ & $\begin{array}{l}0.3676 \\
639.17 \\
1.5205\end{array}$ & $\begin{array}{l}0.3718 \\
645.19 \\
1.5238\end{array}$ & $\begin{array}{l}0.3760 \\
651.24 \\
1.5271\end{array}$ & $\begin{array}{l}0.3801 \\
657.29 \\
1.5304\end{array}$ & $\begin{array}{l}0.3843 \\
663.35 \\
1.5336\end{array}$ \\
\hline 1260 & $\begin{array}{l}V \\
H \\
S\end{array}$ & $\begin{array}{l}0.3455 \\
615.13 \\
1.5061\end{array}$ & $\begin{array}{l}0.3496 \\
621.11 \\
1.5095\end{array}$ & $\begin{array}{l}0.3538 \\
627.11 \\
1.5129\end{array}$ & $\begin{array}{l}0.3579 \\
633.12 \\
1.5163\end{array}$ & $\begin{array}{l}0.3620 \\
639.14 \\
1.5197\end{array}$ & $\begin{array}{l}0.3661 \\
645.17 \\
1.5230\end{array}$ & $\begin{array}{l}0.3702 \\
651.21 \\
1.5263\end{array}$ & $\begin{array}{l}0.3743 \\
657.27 \\
1.5296\end{array}$ & $\begin{array}{l}0.3784 \\
663.33 \\
1.5328\end{array}$ \\
\hline 1280 & $\begin{array}{l}V \\
H \\
S\end{array}$ & $\begin{array}{l}0.3403 \\
615.09 \\
1.5053\end{array}$ & $\begin{array}{l}0.3444 \\
621.08 \\
1.5088\end{array}$ & $\begin{array}{l}0.3484 \\
627.08 \\
1.5122\end{array}$ & $\begin{array}{l}0.3525 \\
633.09 \\
1.5156\end{array}$ & $\begin{array}{l}0.3565 \\
639.11 \\
1.5189\end{array}$ & $\begin{array}{l}0.3606 \\
645.14 \\
1.5223\end{array}$ & $\begin{array}{l}0.3646 \\
651.19 \\
1.5256\end{array}$ & $\begin{array}{l}0.3686 \\
657.24 \\
1.5289\end{array}$ & $\begin{array}{l}0.3727 \\
663.31 \\
1.5321\end{array}$ \\
\hline 1300 & $\begin{array}{l}V \\
H \\
S\end{array}$ & $\begin{array}{l}0.3352 \\
615.06 \\
1.5046\end{array}$ & $\begin{array}{l}0.3392 \\
621.04 \\
1.5080\end{array}$ & $\begin{array}{l}0.3432 \\
627.05 \\
1.5115\end{array}$ & $\begin{array}{l}0.3472 \\
633.06 \\
1.5148\end{array}$ & $\begin{array}{l}0.3512 \\
639.08 \\
1.5182\end{array}$ & $\begin{array}{l}0.3552 \\
645.12 \\
1.5215\end{array}$ & $\begin{array}{l}0.3592 \\
651.16 \\
1.5248\end{array}$ & $\begin{array}{l}0.3632 \\
657.22 \\
1.5281\end{array}$ & $\begin{array}{l}0.3671 \\
663.29 \\
1.5314\end{array}$ \\
\hline+320 & $\begin{array}{l}V \\
H \\
S\end{array}$ & $\begin{array}{l}0.3303 \\
615.02 \\
1.5038\end{array}$ & $\begin{array}{l}0.3343 \\
621.01 \\
1.5073\end{array}$ & $\begin{array}{l}0.3382 \\
627.01 \\
1.5107\end{array}$ & $\begin{array}{l}0.3422 \\
633.03 \\
1.5141\end{array}$ & $\begin{array}{l}0.3461 \\
639.05 \\
1.5175\end{array}$ & $\begin{array}{l}0.3500 \\
645.09 \\
1.5208\end{array}$ & $\begin{array}{l}0.3539 \\
651.14 \\
1.5241\end{array}$ & $\begin{array}{l}0.3579 \\
657.20 \\
1.5274\end{array}$ & $\begin{array}{l}0.3618 \\
663.27 \\
1.5306\end{array}$ \\
\hline 1340 & $\begin{array}{l}V \\
H \\
S\end{array}$ & $\begin{array}{l}0.3256 \\
614.99 \\
1.5031\end{array}$ & $\begin{array}{l}0.3295 \\
620.98 \\
1.5066\end{array}$ & $\begin{array}{l}0.3333 \\
626.98 \\
1.5100\end{array}$ & $\begin{array}{l}0.3372 \\
633.00 \\
1.5134\end{array}$ & $\begin{array}{l}0.3411 \\
639.03 \\
1.5168\end{array}$ & $\begin{array}{l}0.3450 \\
645.06 \\
1.5201\end{array}$ & $\begin{array}{l}0.3488 \\
651.11 \\
1.5234\end{array}$ & $\begin{array}{l}0.3527 \\
657.17 \\
1.5267\end{array}$ & $\begin{array}{l}0.3566 \\
663.24 \\
1.5299\end{array}$ \\
\hline 1360 & $\begin{array}{l}V \\
H \\
S\end{array}$ & $\begin{array}{l}0.3209 \\
614.95 \\
1.5024\end{array}$ & $\begin{array}{l}0.3248 \\
620.95 \\
1.5059\end{array}$ & $\begin{array}{l}0.3286 \\
626.95 \\
1.5093\end{array}$ & $\begin{array}{r}0.3324 \\
632.97 \\
1.5127\end{array}$ & $\begin{array}{l}0.3363 \\
639.00 \\
1.5161\end{array}$ & $\begin{array}{l}0.3401 \\
645.04 \\
1.5194\end{array}$ & $\begin{array}{l}0.3439 \\
651.09 \\
1.5227\end{array}$ & $\begin{array}{l}0.3477 \\
657.15 \\
1.5260\end{array}$ & $\begin{array}{l}0.3515 \\
663.22 \\
1.5292\end{array}$ \\
\hline 1380 & $\begin{array}{l}V \\
H \\
S\end{array}$ & $\begin{array}{l}0.3165 \\
614.92 \\
1.5017\end{array}$ & $\begin{array}{l}0.3202 \\
620.91 \\
1.5052\end{array}$ & $\begin{array}{l}0.3240 \\
626.92 \\
1.5086\end{array}$ & $\begin{array}{l}0.3278 \\
632.94 \\
1.5120\end{array}$ & $\begin{array}{l}0.3316 \\
638.97 \\
1.5154\end{array}$ & $\begin{array}{l}0.3353 \\
645.01 \\
1.5187\end{array}$ & $\begin{array}{l}0.3391 \\
651.06 \\
1.5220\end{array}$ & $\begin{array}{l}0.3429 \\
657.13 \\
1.5253\end{array}$ & $\begin{array}{l}0.3466 \\
663.20 \\
1.5285\end{array}$ \\
\hline 1400 & $\begin{array}{l}V \\
H \\
S\end{array}$ & $\begin{array}{l}0.3121 \\
614.88 \\
1.5010\end{array}$ & $\begin{array}{l}0.3158 \\
620.88 \\
1.5045\end{array}$ & $\begin{array}{l}0.3196 \\
626.89 \\
1.5079\end{array}$ & $\begin{array}{l}0.3233 \\
632.91 \\
1.5113\end{array}$ & $\begin{array}{l}0.3270 \\
638.94 \\
1.5147\end{array}$ & $\begin{array}{l}0.3307 \\
644.98 \\
1.5180\end{array}$ & $\begin{array}{l}0.3344 \\
651.04 \\
1.5213\end{array}$ & $\begin{array}{l}0.3381 \\
657.10 \\
1.5246\end{array}$ & $\begin{array}{l}0.3418 \\
663.18 \\
1.5278\end{array}$ \\
\hline 1450 & $\begin{array}{l}V \\
H \\
S\end{array}$ & $\begin{array}{l}0.3017 \\
614.79 \\
1.4993\end{array}$ & $\begin{array}{l}0.3053 \\
620.80 \\
1.5028\end{array}$ & $\begin{array}{l}0.3089 \\
626.81 \\
1.5062\end{array}$ & $\begin{array}{l}0.3125 \\
632.84 \\
1.5096\end{array}$ & $\begin{array}{l}0.3161 \\
638.87 \\
1.5130\end{array}$ & $\begin{array}{l}0.3197 \\
644.92 \\
1.5163\end{array}$ & $\begin{array}{l}0.3233 \\
650.98 \\
1.5196\end{array}$ & $\begin{array}{l}0.3269 \\
657.05 \\
1.5229\end{array}$ & $\begin{array}{l}0.3305 \\
063.12 \\
1.5262\end{array}$ \\
\hline
\end{tabular}


1.4977

1.5011

626.73

0.3025

0.3060

\subsection{5}

0.3130

0.3164

0.3199 $632.76 \quad 638.80$

644.85

650.91

656.99

663.07

$0.2829 \quad 0.2863 \quad 0.2897$

1550

0.2931

1.5114

1514

1.5180

1.5213

1.5245

1.4961

1.4996

1.5030

632.69

0.2965

1.5064

1.5098

0.2999

$644 \cdot 79$

0.3033

650.85

0.3066

0.3100

1.5164

1.5197

$662 \cdot 02$

$\begin{array}{lll}0.2744 & 0.2777 & 0.2810\end{array}$

614.53

620.55

620.57

0.2843

632.61

0.2876

638.66

0.2909

644.72

0.2942

650.79

0.2974

1.5116

1.5149

656.87

0.3007

$1.5049 \quad 1.5082$

\subsection{4}

$644 \cdot 65$

0.2856

650.73

1.5182

$622.54 \quad 638.59$

1.5101

1.5134

0.2888

656.81

1.5167

0.2919

662.31

$1.4930 \quad 1.4965 \quad 1.5000$

0.2683

1.5068

0.2745

0.2775

0.2806

0.271

644.59

650.66
1.5120

656.75

1.5200

1.4916

1.4951

1. 4985

1. 5019

I. 5053

1.5087

1. 5153

0.2837

$662 \cdot 85$

$0.2518 \quad 0.2549$

614.27

0.2549
620.30

0.2579

0.3600

0.2639

0.2559

625.24

632.39

638.45

644.52

0.2700

650.60

0.2730

1.5165

0.2451

0.2481

0.2510

614.18

620.21

626.26

0.2540

632.31

0.2569

638.38

1.5073

1.5106

656.69

3.2760

0.2598

$644 \cdot 45$

0.2628

650.54

1.513

$\therefore 62.79$

1.5171

1.5059

1. 5092

0.2686 $656.63 \quad 662.74$

0.2388

0.2416

0.2445

0.2474

0.2503

0.2531

644.39

0.2560

1.512

1. 5158

1.4874

1.4909

1.4944

1.4978

1.5012

1.5045

650.4
1.5079

0.2588

656.57

0.2617

662.68

$1.5112 \quad 1.5142$

\subsection{7 \\ 0.2356 \\ 0.2384}

0.2412

0.2440

0.2468

0.2523

$0.25 \equiv 1$

1.486

1.4896

625.10
1.4931

632.16

638.24

644.32

656.51

662.63

1.49651 .4999

1.5032

1.5066

1.5090

1.512 ?

0.2270

0.2298

0.2325

0.2353

0.2380

0.2407

0.2434

0.2462

0.2489

$1.4849 \quad 1.4883$

1.4918

1. 4952

638.16
1.4986

644.25

650.35

656.45

$0.2216 \quad 0.2243$

0.2270

0.2296

0.2323

632.01

638.09

0.2350

644.18

0.2376

1.5086

1. 5119

0.2403

656.39

0.2429

650.28

1.5074 


\begin{tabular}{|c|c|c|c|c|c|c|c|c|c|c|}
\hline \multirow{2}{*}{\multicolumn{2}{|c|}{$\begin{array}{l}\text { PRESSURE } \\
\text { PSIA }\end{array}$}} & \multicolumn{9}{|c|}{ TEMPERATURE } \\
\hline & & 1440 & 1460 & 1480 & 1500 & 1520 & 1540 & 1560 & 1580 & 1600 \\
\hline 14.696 & $\begin{array}{l}V \\
H \\
S\end{array}$ & $\begin{array}{r}31.5172 \\
670.57 \\
1.7383\end{array}$ & $\begin{array}{r}31.8491 \\
676.56 \\
1.7414\end{array}$ & $\begin{array}{r}32.1810 \\
682.56 \\
1.7445\end{array}$ & $\begin{array}{r}32.5128 \\
688.57 \\
1.7476\end{array}$ & $\begin{array}{r}32.8447 \\
694.59 \\
1.7506\end{array}$ & $\begin{array}{r}33.1765 \\
700.63 \\
1.7537\end{array}$ & $\begin{array}{r}33.5084 \\
706.67 \\
1.7567\end{array}$ & $\begin{array}{r}33.8402 \\
712.73 \\
1.7597\end{array}$ & $\begin{array}{r}34.1720 \\
718.80 \\
1.7626\end{array}$ \\
\hline 16 & $\begin{array}{l}V \\
H \\
S\end{array}$ & $\begin{array}{r}28.9500 \\
670.57 \\
1.7345\end{array}$ & $\begin{array}{r}29.2548 \\
676.56 \\
1.7376\end{array}$ & $\begin{array}{r}29.5596 \\
682.56 \\
1.7407\end{array}$ & $\begin{array}{r}29.8645 \\
688.57 \\
1.7438\end{array}$ & $\begin{array}{r}30.1693 \\
694.59 \\
1.7468\end{array}$ & $\begin{array}{r}30.4741 \\
700.62 \\
1.7499\end{array}$ & $\begin{array}{r}30.7789 \\
706.67 \\
1.7529\end{array}$ & $\begin{array}{r}31.0837 \\
712.73 \\
1.7558\end{array}$ & $\begin{array}{r}31.3885 \\
718.80 \\
1.7588\end{array}$ \\
\hline 20 & $\begin{array}{l}V \\
H \\
S\end{array}$ & $\begin{array}{r}23.1633 \\
670.57 \\
1.7244\end{array}$ & $\begin{array}{r}23.4073 \\
676.55 \\
1.7276\end{array}$ & $\begin{array}{r}23.6512 \\
682.55 \\
1.7307\end{array}$ & $\begin{array}{r}23.8951 \\
688.56 \\
1.7338\end{array}$ & $\begin{array}{r}24.1390 \\
694.59 \\
1.7368\end{array}$ & $\begin{array}{r}24.3829 \\
700.62 \\
1.7398\end{array}$ & $\begin{array}{r}24.6267 \\
706.67 \\
1.7428\end{array}$ & $\begin{array}{r}24.8706 \\
712.73 \\
1.7458\end{array}$ & $\begin{array}{r}25.1145 \\
718.80 \\
1.7483\end{array}$ \\
\hline 24 & $\begin{array}{l}V \\
H \\
S\end{array}$ & $\begin{array}{r}19.3056 \\
670.56 \\
1.7162\end{array}$ & $\begin{array}{r}19.5089 \\
676.55 \\
1.7194\end{array}$ & $\begin{array}{r}19.7122 \\
682.55 \\
1.7225\end{array}$ & $\begin{array}{r}19.9155 \\
688.56 \\
1.7256\end{array}$ & $\begin{array}{r}20.1188 \\
694.59 \\
1.7286\end{array}$ & $\begin{array}{r}20.3220 \\
700.62 \\
1.7317\end{array}$ & $\begin{array}{r}20.5253 \\
706.67 \\
1.7347\end{array}$ & $\begin{array}{r}20.7286 \\
712.73 \\
1.7376\end{array}$ & $\begin{array}{r}20.9318 \\
716.30 \\
1.7406\end{array}$ \\
\hline 28 & $\begin{array}{l}V \\
H \\
S\end{array}$ & $\begin{array}{r}16.5501 \\
670.56 \\
1.7093\end{array}$ & $\begin{array}{r}16.7243 \\
676.55 \\
1.7125\end{array}$ & $\begin{array}{r}16.8986 \\
682.55 \\
1.7156\end{array}$ & $\begin{array}{r}17.0729 \\
683.56 \\
1.7186\end{array}$ & $\begin{array}{r}17.2472 \\
694.58 \\
1.7217\end{array}$ & $\begin{array}{r}17.4214 \\
700.62 \\
1.7247\end{array}$ & $\begin{array}{r}17.5957 \\
706.67 \\
1.7277\end{array}$ & $\begin{array}{r}17.7700 \\
712.73 \\
1.7307\end{array}$ & $\begin{array}{r}17.9442 \\
718.20 \\
1.7337\end{array}$ \\
\hline 32 & $\begin{array}{l}V \\
H \\
S\end{array}$ & $\begin{array}{r}14.4834 \\
670.56 \\
1.7033\end{array}$ & $\begin{array}{r}14.6359 \\
676.54 \\
1.7065\end{array}$ & $\begin{array}{r}14.7885 \\
682.54 \\
1.7096\end{array}$ & $\begin{array}{r}14.9410 \\
688.56 \\
1.7126\end{array}$ & $\begin{array}{r}15.0935 \\
694.58 \\
1.7157\end{array}$ & $\begin{array}{r}15.2460 \\
700.62 \\
1.7187\end{array}$ & $\begin{array}{r}15.3985 \\
706.67 \\
1.7217\end{array}$ & $\begin{array}{r}15.5510 \\
712.72 \\
1.7247\end{array}$ & $\begin{array}{r}15.7035 \\
718.79 \\
1.7277\end{array}$ \\
\hline 36 & $\begin{array}{l}V \\
H \\
S\end{array}$ & $\begin{array}{r}12.8760 \\
670.55 \\
1.6980\end{array}$ & $\begin{array}{r}13.0116 \\
676.54 \\
1.7012\end{array}$ & $\begin{array}{r}13.1472 \\
682.54 \\
1.7043\end{array}$ & $\begin{array}{r}13.2828 \\
688.55 \\
1.7074\end{array}$ & $\begin{array}{r}13.4184 \\
694.58 \\
1.7104\end{array}$ & $\begin{array}{r}13.5540 \\
700.62 \\
1.7134\end{array}$ & $\begin{array}{r}13.6896 \\
706.66 \\
1.7165\end{array}$ & $\begin{array}{r}13.8252 \\
712.72 \\
1.7194\end{array}$ & $\begin{array}{r}13.9607 \\
7.18 .79 \\
1.7224\end{array}$ \\
\hline 40 & $\begin{array}{l}V \\
H \\
S\end{array}$ & $\begin{array}{r}11.5901 \\
670.55 \\
1.6933\end{array}$ & $\begin{array}{r}11.7122 \\
676.54 \\
1.6964\end{array}$ & $\begin{array}{r}11.8342 \\
682.54 \\
1.5995\end{array}$ & $\begin{array}{r}11.9563 \\
688.55 \\
1.7026\end{array}$ & $\begin{array}{r}12.0783 \\
694.58 \\
1.7057\end{array}$ & $\begin{array}{r}12.2004 \\
700.61 \\
1.7087\end{array}$ & $\begin{array}{r}12.3224 \\
706.66 \\
1.7117\end{array}$ & $\begin{array}{r}12.4445 \\
712.72 \\
1.7147\end{array}$ & $\begin{array}{r}12.5665 \\
718.79 \\
1.7177\end{array}$ \\
\hline 44 & $\begin{array}{l}V \\
H \\
S\end{array}$ & $\begin{array}{r}10.5380 \\
670.55 \\
1.6890\end{array}$ & $\begin{array}{r}10.6490 \\
676.53 \\
1.6921\end{array}$ & $\begin{array}{r}10.7599 \\
682.54 \\
1.6953\end{array}$ & $\begin{array}{r}10.8709 \\
688.55 \\
1.6983\end{array}$ & $\begin{array}{r}10.9819 \\
694.57 \\
1.7014\end{array}$ & $\begin{array}{r}11.0929 \\
700.61 \\
1.7044\end{array}$ & $\begin{array}{r}11.2039 \\
706.66 \\
1.7074\end{array}$ & $\begin{array}{r}11.3148 \\
712.72 \\
1.7104\end{array}$ & $\begin{array}{r}11.4258 \\
718.79 \\
1.7134\end{array}$ \\
\hline 48 & $\begin{array}{l}V \\
H \\
S\end{array}$ & $\begin{array}{l}9.6612 \\
670.54 \\
1.6851\end{array}$ & $\begin{array}{l}9.7630 \\
676.53 \\
1.6882\end{array}$ & $\begin{array}{l}9.3647 \\
682.53 \\
1.6913\end{array}$ & $\begin{array}{l}9.9665 \\
688.55 \\
1.6944\end{array}$ & $\begin{array}{r}10.0682 \\
694.57 \\
1.5975\end{array}$ & $\begin{array}{r}10.1700 \\
700.61 \\
1.7005\end{array}$ & $\begin{array}{r}10.2717 \\
706.66 \\
1.7035\end{array}$ & $\begin{array}{r}10.3734 \\
712.72 \\
1.7065\end{array}$ & $\begin{array}{r}10.4752 \\
718.79 \\
1.7095\end{array}$ \\
\hline 52 & $\begin{array}{l}V \\
H \\
S\end{array}$ & $\begin{array}{l}8.9193 \\
670.54 \\
1.6815\end{array}$ & $\begin{array}{l}9.0133 \\
676.53 \\
1.6846\end{array}$ & $\begin{array}{l}9.1072 \\
682.53 \\
1.6877\end{array}$ & $\begin{array}{l}9.2012 \\
688.54 \\
1.6908\end{array}$ & $\begin{array}{l}9.2951 \\
694.57 \\
1.6939\end{array}$ & $\begin{array}{l}9.3890 \\
700.61 \\
1.6969\end{array}$ & $\begin{array}{l}9.4830 \\
706.66 \\
1.6999\end{array}$ & $\begin{array}{l}9.5769 \\
712.72 \\
1.7029\end{array}$ & $\begin{array}{l}9.6708 \\
718.79 \\
1.7059\end{array}$ \\
\hline
\end{tabular}


$\begin{array}{ll}V & 8.2834\end{array}$ 8.3707 670.54 676.53 1.6782 1.6813

\subsection{9}

1500

682.53

1.6844

8.5452

1520

1540

1560

1580

1600

7.7323

670.53

7.8138

676.52

7.8952

682.52

688.54
1.6875

8.6324

1.687

1.6906

8.7197

700.61

8.8069

706.66

8.8941

712.72
1.6996

8.9814

1.6966

718.79

$\begin{array}{ll}7.9767 & 8.0581\end{array}$

688.54

694.57

8.1395

700.60

8.2210

706.65

8.3024

712.72

8.3838

1.6844

1.6875

1.6905

1.6935

1.6965

1.6994

6.7698

6.8396

6.9095

6.9793

700.60

7.0491

706.65

7.1190

712.71

7.1888

1.6681

1.6713

1.6744

1.6775

1.6805

1.6835

1.6866

1.6895

118.79

$5.9257 \quad 5.9869 \quad 6.0480$

6.1091

6.1702

6.2314

712.71

6.2925 $676.51 \quad 682.51$

688.53

694.56

700.60

706.65

1.6835

718.78

1.6621

1.6653

1.5684

1.6714

5.1605

5.2148

5.2692

5.3236

5.3779

688.52

694.55

2.6805

5.5410

1.6865

1.6568

1.6600

1.6631

1.6661

1.6692

5.4323

5.4867

706.64

$5.5410 \quad 5.5954$

1.6722

1.6752

712.71

718.78

$4.6461 \quad 4.6950$

670.50

676.49

4.7440

4.7930

4.8410

688.51

694.54

4.8908

4.9398

4. 9887

1.6812

1.6583

1.6614

1.6645

700.59

706.64

5.0377

1.6705

1.6735

718.78

$4.2252 \quad 4.2698$

4.3143

$4 \cdot 3588$

4.4033

4.4478

4.4923

706.64

4.5369

1.6765

1.6478

1.6509

1.6540

1.6571

694.54

700.58

1.6662

712.70

4.5814

718.78

3.8745

3.9154

3.9562

3.9970

4.0378

1.6692

1.6722

1.6438

676.48

682.48

688.50

694.53

4.0787

$4 \cdot 1195$

4.1603

712.70

4.2011

1.6593

1.6623

1.6653

718.77

$3.6909 \quad 3.7286 \quad 3.7663$

3.8040

3.8417

1.6682

$\begin{array}{lll}3.5778 & 3.6155 & 3.6532\end{array}$

$670.47 \quad 675.47 \quad 682.48$

688.50

694.53

700.57

3.8040

3.8794

1.6465

1.6526

1.6557

1.6587

1.6617

718.77

3.4285

3.4635

694.52

3.4985

3.5335

3.5686

3.6036

682.47

688.49

1.6493

1.6523

706.62

712.69

718.77

$3.2011 \quad 3.2338$

688.48

694.52

3.2665

1.6553

1.6583

1.6613

670.45

676.45

682.46

1.6431

1.6462

700.56

3.2992

706.62

3.3319

712.69

3.3645

1.6522

1.6552

1.6582 


\begin{tabular}{|c|c|c|c|c|c|c|c|c|c|c|}
\hline \multirow{2}{*}{\multicolumn{2}{|c|}{$\begin{array}{l}\text { PRESSURE } \\
\text { PSIA }\end{array}$}} & \multicolumn{8}{|c|}{ TEMPERATURE } & \multirow{2}{*}{1600} \\
\hline & & 1440 & 1460 & 1480 & 1500 & 1520 & 1540 & 1560 & 1580 & \\
\hline 160 & $\begin{array}{l}V \\
H \\
S\end{array}$ & $\begin{array}{l}2.9100 \\
670.45 \\
1.6309\end{array}$ & $\begin{array}{l}2.9407 \\
676.44 \\
1.6340\end{array}$ & $\begin{array}{l}2.9714 \\
682.46 \\
1.6371\end{array}$ & $\begin{array}{l}3.0021 \\
688.48 \\
1.6402\end{array}$ & $\begin{array}{l}3.0328 \\
694.51 \\
1.6433\end{array}$ & $\begin{array}{l}3.0634 \\
700.56 \\
1.6463\end{array}$ & $\begin{array}{l}3.0941 \\
706.62 \\
1.6493\end{array}$ & $\begin{array}{l}3.1247 \\
712.69 \\
1.6523\end{array}$ & $\begin{array}{l}3.1554 \\
718.77 \\
1.6553\end{array}$ \\
\hline 170 & $\begin{array}{l}V \\
H \\
S\end{array}$ & $\begin{array}{l}2.7398 \\
670.44 \\
1.6281\end{array}$ & $\begin{array}{l}2.7687 \\
676.44 \\
1.6313\end{array}$ & $\begin{array}{l}2.7976 \\
682.45 \\
1.6344\end{array}$ & $\begin{array}{l}2.8265 \\
688.47 \\
1.6375\end{array}$ & $\begin{array}{l}2.8554 \\
694.51 \\
1.6405\end{array}$ & $\begin{array}{l}2.8843 \\
700.55 \\
1.6436\end{array}$ & $\begin{array}{l}2.9131 \\
706.61 \\
1.6466\end{array}$ & $\begin{array}{l}2.9420 \\
712.68 \\
1.6496\end{array}$ & $\begin{array}{l}2.9709 \\
718.76 \\
1.6525\end{array}$ \\
\hline 180 & $\begin{array}{l}V \\
H \\
S\end{array}$ & $\begin{array}{l}2.5885 \\
670.43 \\
1.6255\end{array}$ & $\begin{array}{l}2.6158 \\
676.43 \\
1.6287\end{array}$ & $\begin{array}{l}2.6431 \\
682.44 \\
1.5318\end{array}$ & $\begin{array}{l}2.6704 \\
688.47 \\
1.5349\end{array}$ & $\begin{array}{l}2.6977 \\
694.50 \\
1.6379\end{array}$ & $\begin{array}{l}2.7250 \\
700.55 \\
1.6410\end{array}$ & $\begin{array}{l}2.7523 \\
705.61 \\
1.6440\end{array}$ & $\begin{array}{l}2.7796 \\
712.68 \\
1.6470\end{array}$ & $\begin{array}{l}2.8068 \\
718.76 \\
1.6499\end{array}$ \\
\hline 190 & $\begin{array}{l}V \\
H \\
\text { S }\end{array}$ & $\begin{array}{l}2.4532 \\
670.42 \\
1.6231\end{array}$ & $\begin{array}{l}2.4790 \\
676.42 \\
1.6262\end{array}$ & $\begin{array}{l}2.5049 \\
682.42 \\
1.5292\end{array}$ & $\begin{array}{l}2.5308 \\
688.46 \\
1.6374\end{array}$ & $\begin{array}{l}2.5566 \\
694.50 \\
1.6355\end{array}$ & $\begin{array}{l}2.5825 \\
700.55 \\
1.6385\end{array}$ & $\begin{array}{l}2.6084 \\
706.61 \\
1.6415\end{array}$ & $\begin{array}{l}2.6342 \\
712.68 \\
1.6445\end{array}$ & $\begin{array}{l}2.6601 \\
718.76 \\
1.6475\end{array}$ \\
\hline 200 & $\begin{array}{l}V \\
H \\
S\end{array}$ & $\begin{array}{l}2.3313 \\
670.41 \\
1.6208\end{array}$ & $\begin{array}{l}2.3559 \\
676.41 \\
1.6239\end{array}$ & $\begin{array}{l}2.2805 \\
682.43 \\
1.6270\end{array}$ & $\begin{array}{l}2.4051 \\
688.45 \\
1.6301\end{array}$ & $\begin{array}{l}2.4297 \\
694.49 \\
1.6332\end{array}$ & $\begin{array}{l}2.4543 \\
700.54 \\
1.6362\end{array}$ & $\begin{array}{l}2.4788 \\
706.60 \\
1.6392\end{array}$ & $\begin{array}{l}2.5034 \\
712.67 \\
1.6422\end{array}$ & $\begin{array}{l}2.5280 \\
718.76 \\
1.6452\end{array}$ \\
\hline 220 & $\begin{array}{l}V \\
H \\
S\end{array}$ & $\begin{array}{l}2.1209 \\
670.39 \\
1.6165\end{array}$ & $\begin{array}{l}2.1433 \\
675.40 \\
1.6196\end{array}$ & $\begin{array}{l}2.1656 \\
682.41 \\
1.6277\end{array}$ & $\begin{array}{l}2.2830 \\
688.44 \\
1.6258\end{array}$ & $\begin{array}{l}2.2104 \\
694.48 \\
1.6289\end{array}$ & $\begin{array}{l}2.2327 \\
700.53 \\
1.6319\end{array}$ & $\begin{array}{l}2.2551 \\
706.59 \\
1.6349\end{array}$ & $\begin{array}{l}2.2774 \\
712.67 \\
1.6379\end{array}$ & $\begin{array}{l}2.2998 \\
718.75 \\
1.6409\end{array}$ \\
\hline 240 & $\begin{array}{l}V \\
H \\
S\end{array}$ & $\begin{array}{l}1.9455 \\
670.38 \\
1.6125\end{array}$ & $\begin{array}{l}1.9661 \\
675.38 \\
1.6157\end{array}$ & $\begin{array}{l}1.2856 \\
682.40 \\
1.6188\end{array}$ & $\begin{array}{l}2.0071 \\
68.8 .43 \\
1.6219\end{array}$ & $\begin{array}{l}2.0276 \\
694.47 \\
1.6249\end{array}$ & $\begin{array}{l}2.0481 \\
700.52 \\
1.6280\end{array}$ & $\begin{array}{l}2.0686 \\
706.59 \\
1.6310\end{array}$ & $\begin{array}{l}2.0891 \\
712.66 \\
1.6340\end{array}$ & $\begin{array}{l}2.1097 \\
718.75 \\
1.6369\end{array}$ \\
\hline 260 & $\begin{array}{l}V \\
H \\
S\end{array}$ & $\begin{array}{l}1.7971 \\
670.36 \\
1.6089\end{array}$ & $\begin{array}{l}1.8161 \\
676.37 \\
1.6120\end{array}$ & $\begin{array}{l}1.8351 \\
682.39 \\
1.6151\end{array}$ & $\begin{array}{l}1.8540 \\
688.42 \\
1.6182\end{array}$ & $\begin{array}{l}1.8730 \\
694.46 \\
1.6213\end{array}$ & $\begin{array}{l}1.8919 \\
700.51 \\
1.6243\end{array}$ & $\begin{array}{l}1.9109 \\
706.58 \\
1.6273\end{array}$ & $\begin{array}{l}1.9298 \\
712.66 \\
1.6303\end{array}$ & $\begin{array}{l}1.9488 \\
718.74 \\
1.6333\end{array}$ \\
\hline 280 & $\begin{array}{l}V \\
H \\
S\end{array}$ & $\begin{array}{l}1.6699 \\
670.34 \\
1.6055\end{array}$ & $\begin{array}{l}1.6876 \\
676.35 \\
1.6087\end{array}$ & $\begin{array}{l}1.7052 \\
682.37 \\
1.6118\end{array}$ & $\begin{array}{l}1.7228 \\
688.41 \\
1.6149\end{array}$ & $\begin{array}{l}1.7404 \\
694.45 \\
1.6179\end{array}$ & $\begin{array}{l}1.7580 \\
700.51 \\
1.6210\end{array}$ & $\begin{array}{l}1.7756 \\
706.57 \\
1.6240\end{array}$ & $\begin{array}{l}1.7931 \\
712.64 \\
1.6270\end{array}$ & $\begin{array}{l}1.8107 \\
718.73 \\
1.6299\end{array}$ \\
\hline 300 & $\begin{array}{l}V \\
H \\
S\end{array}$ & $\begin{array}{l}1.5595 \\
670.32 \\
1.6024\end{array}$ & $\begin{array}{l}1.5760 \\
676.33 \\
1.6055\end{array}$ & $\begin{array}{l}1.5924 \\
682.35 \\
1.6086\end{array}$ & $\begin{array}{l}1.6089 \\
688.38 \\
1.6117\end{array}$ & $\begin{array}{l}1.6254 \\
694.43 \\
1.6148\end{array}$ & $\begin{array}{l}1.6418 \\
700.49 \\
1.6178\end{array}$ & $\begin{array}{l}1.6583 \\
706.55 \\
1.6209\end{array}$ & $\begin{array}{l}1.6747 \\
712.63 \\
1.6238\end{array}$ & $\begin{array}{l}1.6911 \\
718.72 \\
1.6268\end{array}$ \\
\hline 320 & $\begin{array}{l}V \\
H \\
S\end{array}$ & $\begin{array}{l}1.4630 \\
670.30 \\
1.5994\end{array}$ & $\begin{array}{l}1.4785 \\
676.31 \\
1.6026\end{array}$ & $\begin{array}{l}1.4939 \\
682.33 \\
1.5057\end{array}$ & $\begin{array}{l}1.5094 \\
688.37 \\
1.6088\end{array}$ & $\begin{array}{l}1.5248 \\
694.42 \\
1.6119\end{array}$ & $\begin{array}{l}1.5403 \\
700.48 \\
1.6149\end{array}$ & $\begin{array}{l}1.5557 \\
706.55 \\
1.6179\end{array}$ & $\begin{array}{l}1.5711 \\
712.63 \\
1.6209\end{array}$ & $\begin{array}{l}1.5865 \\
718.72 \\
1.6239\end{array}$ \\
\hline
\end{tabular}


340

360

$38 n$

400

420

447

460

480

500

520

$540 \quad \mathrm{~V}$
$\mathrm{H}$
$\mathrm{S}$

\begin{tabular}{|c|c|}
\hline 1440 & 1460 \\
\hline $\begin{array}{l}1.3779 \\
670.28 \\
1.5967\end{array}$ & $\begin{array}{l}1.3925 \\
676.29 \\
1.5998\end{array}$ \\
\hline $\begin{array}{l}1.3022 \\
670.26 \\
1.5941\end{array}$ & $\begin{array}{l}1.3160 \\
676.28 \\
1.5972\end{array}$ \\
\hline $\begin{array}{l}1.2345 \\
670.24 \\
1.5916\end{array}$ & $\begin{array}{l}1.2476 \\
676.26 \\
1.5947\end{array}$ \\
\hline $\begin{array}{l}1.1736 \\
670.23 \\
1.5893\end{array}$ & $\begin{array}{l}1.1860 \\
676.24 \\
1.5924\end{array}$ \\
\hline $\begin{array}{l}1.1184 \\
670.21 \\
1.5870\end{array}$ & $\begin{array}{l}1.1303 \\
676.23 \\
1.5902\end{array}$ \\
\hline $\begin{array}{l}1.0683 \\
670.19 \\
1.5849\end{array}$ & $\begin{array}{l}1.0796 \\
676.21 \\
1.5881\end{array}$ \\
\hline $\begin{array}{l}1.0225 \\
670.17 \\
1.5829\end{array}$ & $\begin{array}{l}1.0334 \\
676.19 \\
1.5860\end{array}$ \\
\hline $\begin{array}{l}0.9806 \\
670.15 \\
1.5809\end{array}$ & $\begin{array}{l}0.9910 \\
676.18 \\
1.5841\end{array}$ \\
\hline $\begin{array}{l}0.9420 \\
670.13 \\
1.5790\end{array}$ & $\begin{array}{l}0.9519 \\
676.16 \\
1.5822\end{array}$ \\
\hline $\begin{array}{l}0.9063 \\
670.12 \\
1.5772\end{array}$ & $\begin{array}{l}0.9159 \\
676.114 \\
1.5804\end{array}$ \\
\hline $\begin{array}{l}0.8733 \\
670.10 \\
1.5755\end{array}$ & $\begin{array}{l}0.8826 \\
676.13 \\
1.5787\end{array}$ \\
\hline
\end{tabular}

\section{TEMPERATURE}

$1500 \quad 1520$

1480

1.4216

682070

1.6029

688.36

1.4361

694.41

1.6060

1.6091

\subsection{8}

682.30

1.3435

688.34

1.3572

694.39

1.6065

1.2606

682.29

1.5979

1.2737

1.2737
688.33

1.6020

1.2867

694.38

1.6040

1.1984
682.27

682.27
1.5955

1.2108

683.32

1. 2232

694.37

1.6017

1.1421

682.26

1.1530

688.30

1.1657

1.5933

1.5964

$\leqslant 94.36$

1.0909

682.24

1.5912

1.1022

683.29

1.5943

1. 1135

694.35

1. 5973

1.0442

682.23

1.0550

688.28

1.5922

1.0658

694.33

1.5953

1.0013

1.0117

688.26

1.0220

694.32

1.5934

1.5872

1.5903

0.9619

0.9719

688.25

0.9818

694.31

1.5853

1. 5884

1.5915

0.9255

682.18

1.5835

0.9351

688.23

I. 5866

0.9447

694.30

I. 5897

0.8918

682.17

0.9011

688.22

0.9103

694.28

I. 5849

1.5880
1540

1.4506

700.47

1.6121

1.3710

700.46

1.6095

1.2997

700.44
1.6071

1.2356

700.43

1.6047

1.1775

700.42

1.6025

1. 1248

700.41

1.6004

1.0766

700.40

I. 5984

1.0324

700.39

1.5964

0.9918

700.38

1.5945

0.9543

700.37

1.5928

0.9195

$700 \cdot 36$

1.5910
1560

1580

1.4652

706.54

1.4797

712.62

$1.6152 \quad 1.6182$

1.6182

1.3847

706.53

1.3985

712.61

$1.6126 \quad 1.6156$

$1.3127 \quad 1.3258$

$706.52 \quad 712.60$

$1.6101 \quad 1.6131$

1.2480

705.51

1.2603

712.60

1.6108

1.1893

706.50

1.2011

712.59

1.6085

1.1360

1.1473

$706.49 \quad 712.58$

$1.6034 \quad 1.6064$

1.6064

$1.0874 \quad 1.0982$

$706.48 \quad 712.57$

1.6044

1.0428

706.47

1.0531

712.57

1.6024

1.0017

706.46

1.0117

712.56

$1.5976 \quad 1.6006$

0.9734

0.9638

706.45

1.5958

712.55
1.5988

0.9287

706.45

0.9380

1.5940

712.54
1.5970

718.65

1.6000
1600

1.4942

$18 \cdot 71$

1.4122

718.71

1.6185

1.3388

$718 \cdot 70$

1.6161

1.2727

118.69

1.2129

$718 \cdot 69$

1.6115

1.1586

718.68

1.6094

1.1089

118.67

1.0634

718.67

1.0216

718.66

1.6035

0.9830

718.65

1.6018 


\begin{tabular}{|c|c|c|c|c|c|c|c|c|c|c|}
\hline \multirow{2}{*}{\multicolumn{2}{|c|}{$\begin{array}{l}\text { PRESSURE } \\
\text { PSIA }\end{array}$}} & \multicolumn{8}{|c|}{ TEMPERATURE } & \multirow[b]{2}{*}{1600} \\
\hline & & 1440 & 1460 & 1480 & 1500 & & 1540 & 2560 & 1580 & \\
\hline 560 & $\begin{array}{l}V \\
H \\
S\end{array}$ & $\begin{array}{l}0.8427 \\
670.08 \\
1.5738\end{array}$ & $\begin{array}{l}0.8516 \\
676.11 \\
1.5770\end{array}$ & $\begin{array}{l}0.3605 \\
682.15 \\
1.5801\end{array}$ & $\begin{array}{l}0.8694 \\
688.21 \\
1.5832\end{array}$ & $\begin{array}{l}0.8783 \\
694.27 \\
1.5863\end{array}$ & $\begin{array}{l}0.8873 \\
700.35 \\
1.5894\end{array}$ & $\begin{array}{l}0.8962 \\
706.44 \\
1.5924\end{array}$ & $\begin{array}{l}0.9051 \\
712.53 \\
1.5954\end{array}$ & $\begin{array}{l}0.9139 \\
718.64 \\
1.5984\end{array}$ \\
\hline 580 & $\begin{array}{l}V \\
H \\
S\end{array}$ & $\begin{array}{l}0.8141 \\
670.06 \\
1.5722\end{array}$ & $\begin{array}{l}0.8228 \\
676.09 \\
1.5754\end{array}$ & $\begin{array}{l}0.8314 \\
682.14 \\
1.5785\end{array}$ & $\begin{array}{l}0.8400 \\
688.19 \\
1.5816\end{array}$ & $\begin{array}{l}0.8486 \\
694.26 \\
1.5847\end{array}$ & $\begin{array}{l}0.8572 \\
700.34 \\
1.5377\end{array}$ & $\begin{array}{l}0.8658 \\
706.43 \\
1.5908\end{array}$ & $\begin{array}{l}0.8744 \\
712.53 \\
1.5938\end{array}$ & $\begin{array}{l}0.8830 \\
718.64 \\
1.5968\end{array}$ \\
\hline 600 & $\begin{array}{l}V \\
H \\
S\end{array}$ & $\begin{array}{l}0.7875 \\
670.04 \\
1.5707\end{array}$ & $\begin{array}{l}0.7959 \\
676.08 \\
1.5738\end{array}$ & $\begin{array}{l}0.8042 \\
682.12 \\
1.5769\end{array}$ & $\begin{array}{l}0.8125 \\
688.18 \\
1.5801\end{array}$ & $\begin{array}{l}0.8209 \\
694.25 \\
1.5831\end{array}$ & $\begin{array}{l}0.8292 \\
700.33 \\
1.5362\end{array}$ & $\begin{array}{l}0.8375 \\
706.42 \\
1.5892\end{array}$ & $\begin{array}{l}0.8458 \\
712.52 \\
1.5922\end{array}$ & $\begin{array}{l}0.8541 \\
718.63 \\
1.5952\end{array}$ \\
\hline 620 & $\begin{array}{l}V \\
H \\
S\end{array}$ & $\begin{array}{l}0.7626 \\
670.02 \\
1.5691\end{array}$ & $\begin{array}{l}0.7707 \\
676.06 \\
1.5723\end{array}$ & $\begin{array}{l}0.7788 \\
682.11 \\
1.5754\end{array}$ & $\begin{array}{l}0.7868 \\
688.17 \\
1.5735\end{array}$ & $\begin{array}{l}0.7949 \\
694.24 \\
1.5816\end{array}$ & $\begin{array}{l}0.8030 \\
700.32 \\
1.5847\end{array}$ & $\begin{array}{l}0.8110 \\
706.41 \\
1.5877\end{array}$ & $\begin{array}{l}0.8191 \\
712.51 \\
1.5907\end{array}$ & $\begin{array}{l}0.8271 \\
718.62 \\
1.5937\end{array}$ \\
\hline 640 & $\begin{array}{l}y \\
H \\
s\end{array}$ & $\begin{array}{l}0.7392 \\
670.00 \\
1.5677\end{array}$ & $\begin{array}{l}0.7471 \\
676.04 \\
1.5708\end{array}$ & $\begin{array}{l}0.7549 \\
682.09 \\
1.5740\end{array}$ & $\begin{array}{l}0.7627 \\
582.15 \\
1.5771\end{array}$ & $\begin{array}{l}0.7705 \\
594.22 \\
1.5802\end{array}$ & $\begin{array}{l}0.7784 \\
700.30 \\
1.5832\end{array}$ & $\begin{array}{l}0.7862 \\
706.40 \\
1.5862\end{array}$ & $\begin{array}{l}0.7940 \\
712.50 \\
1.5893\end{array}$ & $\begin{array}{l}0.8018 \\
718.61 \\
1.5922\end{array}$ \\
\hline 660 & $\begin{array}{l}V \\
H \\
S\end{array}$ & $\begin{array}{l}0.7173 \\
660.98 \\
1.5663\end{array}$ & $\begin{array}{l}0.7249 \\
676.02 \\
1.5694\end{array}$ & $\begin{array}{l}0.7325 \\
682.08 \\
1.5726\end{array}$ & $\begin{array}{l}0.7401 \\
682.14 \\
1.5757\end{array}$ & $\begin{array}{l}0.7477 \\
694.21 \\
3.5787\end{array}$ & $\begin{array}{l}0.7553 \\
700.29 \\
1.5818\end{array}$ & $\begin{array}{l}0.7628 \\
706.39 \\
1.5848\end{array}$ & $\begin{array}{l}0.7704 \\
712.49 \\
1.5878\end{array}$ & $\begin{array}{l}0.7780 \\
718.61 \\
1.5908\end{array}$ \\
\hline 680 & $\begin{array}{l}V \\
H \\
S\end{array}$ & $\begin{array}{l}0.6966 \\
669.97 \\
1.5649\end{array}$ & $\begin{array}{l}0.7040 \\
676.01 \\
1.5680\end{array}$ & $\begin{array}{l}0.7114 \\
682.06 \\
1.5712\end{array}$ & $\begin{array}{l}0.7188 \\
688.12 \\
1.5743\end{array}$ & $\begin{array}{l}0.7261 \\
694.20 \\
1.5774\end{array}$ & $\begin{array}{l}0.7335 \\
700.28 \\
1.5804\end{array}$ & $\begin{array}{l}0.7409 \\
706.38 \\
1.5835\end{array}$ & $\begin{array}{l}0.7482 \\
712.48 \\
1.5865\end{array}$ & $\begin{array}{l}0.7556 \\
718.60 \\
1.5894\end{array}$ \\
\hline 700 & $\begin{array}{l}V \\
H \\
S\end{array}$ & $\begin{array}{l}0.6771 \\
669.95 \\
1.5635\end{array}$ & $\begin{array}{l}0.6843 \\
675.99 \\
1.5667\end{array}$ & $\begin{array}{l}0.5915 \\
682.04 \\
1.5698\end{array}$ & $\begin{array}{l}0.6987 \\
688.11 \\
1.5729\end{array}$ & $\begin{array}{l}0.7058 \\
694.18 \\
1.5760\end{array}$ & $\begin{array}{l}0.7130 \\
700.27 \\
1.5791\end{array}$ & $\begin{array}{l}0.7202 \\
706.37 \\
1.5821\end{array}$ & $\begin{array}{l}0.7273 \\
712.48 \\
1.5851\end{array}$ & $\begin{array}{l}0.7345 \\
718.59 \\
1.5881\end{array}$ \\
\hline 720 & $\begin{array}{l}V \\
H \\
S\end{array}$ & $\begin{array}{l}0.6588 \\
669.93 \\
1.5622\end{array}$ & $\begin{array}{l}0.6657 \\
675.97 \\
1.5654\end{array}$ & $\begin{array}{l}0.5727 \\
682.03 \\
1.5685\end{array}$ & $\begin{array}{l}0.6797 \\
688.09 \\
1.5716\end{array}$ & $\begin{array}{l}0.6867 \\
694.17 \\
1.5747\end{array}$ & $\begin{array}{l}0.6936 \\
700.26 \\
1.5778\end{array}$ & $\begin{array}{l}0.7006 \\
706.36 \\
1.5808\end{array}$ & $\begin{array}{l}0.7076 \\
712.47 \\
1.5838\end{array}$ & $\begin{array}{l}0.7145 \\
718.59 \\
1.5868\end{array}$ \\
\hline 740 & $\begin{array}{l}V \\
H \\
S\end{array}$ & $\begin{array}{l}0.6413 \\
669.91 \\
1.5610\end{array}$ & $\begin{array}{l}0.6481 \\
675.96 \\
1.5641\end{array}$ & $\begin{array}{l}0.5549 \\
682.01 \\
1.5673\end{array}$ & $\begin{array}{l}0.6617 \\
688.08 \\
1.5704\end{array}$ & $\begin{array}{l}0.6685 \\
694.16 \\
1.5735\end{array}$ & $\begin{array}{l}0.6753 \\
700.25 \\
1.5765\end{array}$ & $\begin{array}{l}0.6821 \\
706.35 \\
1.5796\end{array}$ & $\begin{array}{l}0.6889 \\
712.46 \\
1.5826\end{array}$ & $\begin{array}{l}0.6957 \\
718.58 \\
1.5855\end{array}$ \\
\hline 760 & $\begin{array}{l}V \\
H \\
S\end{array}$ & $\begin{array}{l}0.6249 \\
669.89 \\
1.5597\end{array}$ & $\begin{array}{l}0.6315 \\
675.94 \\
1.5629\end{array}$ & $\begin{array}{l}0.6381 \\
682.00 \\
1.5660\end{array}$ & $\begin{array}{l}0.6447 \\
688.07 \\
1.5691\end{array}$ & $\begin{array}{l}0.6513 \\
694.15 \\
1.5722\end{array}$ & $\begin{array}{l}0.6580 \\
700.24 \\
1.5753\end{array}$ & $\begin{array}{l}0.6646 \\
706.34 \\
1.5783\end{array}$ & $\begin{array}{l}0.6712 \\
712.45 \\
1.5813\end{array}$ & $\begin{array}{l}0.6778 \\
718.57 \\
1.5843\end{array}$ \\
\hline
\end{tabular}




1460

0.6092

669.87

1.5585

0.6157

675.92

1.5617

0.5943

800

669.85

1.5574

0.6007

675.90

1.5605

0.5802

669.83

0.5864

675.89

1.5594

0.5667

669.81

.0 .5727

675.87

1.5582

0.5539

669.80

1.5540

0.5598

675.85

1.5572

0.5416

669.78

1. 5529

0.5474

675.83

1.5561

0.5299

669.76

0.5355

675.82

1.5550

$0.5187 \quad 0.5242$

$669.74 \quad 675.80$

1. 5508

1.5540

0.5080

669.72

1.5498

0.5134

675.78

1.5530

0.4977

669.70

0.5030

675.76

1.5488

1.5520

0.4878
659.68

1.5479

0.4930
675.74

1.5511
TEMPERATURE

$1480 \quad 1500 \quad 1520$

0.6221

681.98

1.5648

0.6286
688.05

688.05
1.5679

0.6070

681.96

1.5637

0.6133

688.04

1.5668

0.5925
681.95

1.5625

0.5987

688.02

1.5656

0.5788

681.93

0.5848

688.01

1.5645

0.5656

681.92

0.5715

687.99

1.5634

0.5531

681.90

1.5592

0.5589

637.98

1.5623

0.5412

681.88

0.5468

637.96

1.5613

0.5297

0.5352

687.95

1.5572

1.5603

0.5188

681.85

1.5561

0.5242

687.93

1.5593

0.5083

681.84

0.5135

687.92

1.5583

$0.4982 \quad 0.5034$

681.82

1.5542
0.6350

694.13

1.5710

0.6196

694.12

1.5699

0.6048

694.11

1.5687

0.5908

694.09

1. 5676

0.5774

694.08

1. 5665

0.5646

694.07

1.5654

0.5524

694.05

1.5644

0.5407

694.04

1.5634

0.5295

694.03

1. 5624

0.5188

694.01

1. 5614

0.5085

694.00

1.5604

\section{0}

1560

1580

1600

$0.6415 \quad 0.6479$

700.23

1.5741

706.33

1. 5771

0.6258

700.21

1.5729

0.6321

706.32

1.5759

0.6110

700.20

1. 5718

0.5968

700.19

1.5707

0.5833

700.18

1.5596

0.5704

700.17

1.5685

0.5580

700.16

1.5674

0.5462

700.14

1.5664

0.5349

700.13

1.5654

0.5241

700.12

1.5644

0.5137

700.11

I. 5635
0.6171

706.31

1.5748

0.6028

706.30

1.5737

0.5891

706.29

1.5726

0.5761

706.28

1.5715

0.5636

706.27

1.5705

0.5517

706.26

1.5695

0.5403

706.25

I. 5685

0.5294

706.24

1. 5675

0.5189

706.22

1. 5665
0.6544

712.44

1.5801

0.6608

718.56

$\begin{array}{ll}0.6384 & 0.6447\end{array}$

$712.43 \quad 718.56$

$1.5790 \quad 1.5819$

$0.6232 \quad 0.6294$

$712.42 \quad 718.55$

$1.5778 \quad 1.5808$

$0.6088 \quad 0.6148$

$712.42 \quad 7 * 8.54$

$1.5767 \quad 1.5797$

$0.5950 \quad 0.6008$

$712.41 \quad 718.53$

$1.5756 \quad 1.5786$

$0.5818 \quad 0.5875$

$712.40 \quad 718.53$

$1.5745 \quad 1.5775$

$\begin{array}{lll}0.5692 & 0.5748\end{array}$

$712.39 \quad 718.52$

$1.5735 \quad 1.5765$

$\begin{array}{lll}0.5572 & 0.5627\end{array}$

$712.38 \quad 718.51$

1.57251 .5755

$0.5457 \quad 0.5517$

$712.37 \quad 718.50$

$1.5715 \quad 1.5745$

$\begin{array}{ll}0.5346 & 0.5399\end{array}$

$712.36 \quad 718.50$

$1.5705 \quad 1.5735$

$0.5240 \quad 0.5292$

$712.35 \quad 718.49$

1.56951 .5725 


\begin{tabular}{|c|c|c|c|c|c|c|c|c|c|c|}
\hline \multirow{2}{*}{\multicolumn{2}{|c|}{$\begin{array}{l}\text { PRESSURE } \\
\text { PSIA }\end{array}$}} & \multicolumn{8}{|c|}{ TEMPERATURE } & \multirow[b]{2}{*}{1600} \\
\hline & & 1440 & 1460 & 1480 & 1500 & 1520 & 1540 & 1560 & 1580 & \\
\hline & v & 0.4783 & 0.4834 & 0.4885 & 0.4936 & 0.4987 & 0.5037 & 0.5088 & 0.5139 & 0.5189 \\
\hline 1000 & $H$ & 669.66 & 675.73 & 681.80 & 687.89 & 693.99 & $700 \cdot 10$ & 706.21 & $712 \cdot 34$ & 718.48 \\
\hline & & 1.5469 & 1.5501 & 1.5533 & 1.5564 & 1.5595 & 1.5625 & 1.5656 & 1.5686 & $1.57: 6$ \\
\hline \multirow{3}{*}{1020} & $v$ & 0.4692 & 0.4742 & 0.4792 & 0.4842 & 0.4892 & 0.4942 & 0.4991 & 0.5041 & $0.509 i$ \\
\hline & H & 669.64 & 675.71 & 681.79 & 687.88 & 593.97 & $700 \cdot 08$ & 706.20 & 712.33 & 718.47 \\
\hline & $s$ & 1.5460 & 1.5492 & 1.5523 & 1.5555 & 1.5585 & 1.5616 & 1.5647 & 1.5677 & 1.5707 \\
\hline \multirow{3}{*}{1040} & v & 0.4605 & 0.4654 & 0.4703 & 0.4752 & 0.4801 & 0.4849 & 0.4898 & 0.4947 & 0.4996 \\
\hline & $r$ & 669.62 & 675.69 & 681.77 & 587.86 & 693.96 & 700.07 & 706.19 & 712.32 & $718 \cdot 46$ \\
\hline & $s$ & 1.5451 & 1.5483 & 1.5514 & 1.5545 & 1.5575 & 1.5607 & 1.5638 & 1.5668 & $1 \cdot 5695$ \\
\hline \multirow{3}{*}{1050} & v & 0.4520 & 0.4569 & 0.4517 & 0.4665 & 0.4713 & 0.4761 & 0.4309 & 0.4856 & 0.4904 \\
\hline & $H$ & 669.50 & 675.67 & 581.75 & 687.85 & 693.95 & 700.06 & 706.18 & 712.31 & 712.46 \\
\hline & $s$ & 1.5442 & 1.5474 & 1.5505 & 1.5537 & 1.5567 & 1.5598 & 1.5629 & 1.5659 & 1.5689 \\
\hline \multirow{3}{*}{1080} & $v$ & 0.4439 & 0.4487 & 0.4534 & 0.4581 & 0.4628 & 0.4675 & 0.4722 & 0.4769 & 0.4216 \\
\hline & $H$ & $669.5 \varepsilon$ & 675.65 & 681.74 & 687.83 & 693.93 & 700.05 & 706.17 & 712.30 & $718 \cdot 45$ \\
\hline & $s$ & 1.5433 & 1.5455 & 1.3497 & 1.5528 & 1.5559 & 1.5589 & 1.5620 & 1.5650 & 1.5630 \\
\hline \multirow{3}{*}{1100} & V & 0.4361 & 0.4408 & 0.4454 & 0.4500 & 0.4547 & 0.4593 & 0.4639 & 0.4685 & 0.4732 \\
\hline & $H$ & $669.5 E$ & 675.64 & 681.72 & 687.81 & 693.92 & 700.03 & 766.16 & 712.29 & 718.44 \\
\hline & $s$ & 1.5425 & 1.5456 & 1.5488 & 1.5519 & 1.5550 & 1.5581 & 1.5611 & 1.5542 & $2=5571$ \\
\hline \multirow{3}{*}{1120} & v & 0.4286 & 0.4331 & 0.4377 & $0.44 \geq 3$ & 0.4468 & 0.4514 & 0.4559 & 0.4604 & 0.4650 \\
\hline & $H$ & 669.54 & 675.62 & 681.70 & 587.80 & 593.91 & 700.02 & 706.15 & 712.28 & 718.43 \\
\hline & $s$ & 1.5416 & 1.5448 & 1.5479 & 1.5511 & 1.5542 & 1.5572 & 1.5603 & 1.5633 & 1.5663 \\
\hline \multirow{3}{*}{1140} & $v^{\prime}$ & 0.4213 & 0.4258 & 0.4303 & 0.4347 & 0.4392 & 0.4437 & 0.4482 & 0.4526 & 0.4571 \\
\hline & $H$ & 669.52 & 675.60 & 681.69 & 687.78 & 693.89 & 700.01 & 706.14 & 712.28 & $718 \div 2$ \\
\hline & $s$ & 1.5408 & 1.5440 & 1.5471 & 1.5502 & 1.5533 & 1.5564 & 1.5595 & 1.5625 & 1.5655 \\
\hline \multirow{3}{*}{1160} & v & $c .4143$ & 0.4187 & 0.4231 & 0.4275 & 0.4319 & 0.4363 & 0.4407 & 0.4451 & 0.4495 \\
\hline & $H_{i}$ & $669.5 \mathrm{C}$ & 675.58 & 681.67 & 687.77 & 593.88 & 700.00 & 706.13 & 712.27 & 718.41 \\
\hline & $s$ & 1.5400 & 1.5431 & 1.5463 & 1.5494 & i. 5525 & 1.5556 & 1.5585 & 1.5617 & 1.5647 \\
\hline \multirow{3}{*}{1180} & v & 0.4075 & 0.4118 & 0.4162 & 0.4205 & 0.4248 & 0.4292 & 0.4335 & 0.4378 & 0.4421 \\
\hline & $H$ & 669.48 & 675.56 & 681.65 & 587.75 & 693.86 & 699.99 & 706.12 & 712.26 & $718 \cdot 41$ \\
\hline & $s$ & 1.5392 & 1.5423 & 1.5455 & 1.5486 & 1.5517 & 1.5548 & 1.5578 & 1.5609 & 1.5539 \\
\hline \multirow{2}{*}{1200} & V & 0.4009 & 0.4052 & 0.4095 & 0.4137 & $\begin{array}{l}0.4100 \\
69385\end{array}$ & 0.4222 & 0.4265 & 0.4307 & 0.4350 \\
\hline & $\begin{array}{l}H \\
S\end{array}$ & 1.5384 & 1.5415 & 1.5447 & 1.5478 & 1.5509 & $1.55<0$ & $\begin{array}{l}106.10 \\
1.5570\end{array}$ & $\begin{array}{l}712.25 \\
1.5601\end{array}$ & $\begin{array}{r}18.40 \\
1.5631\end{array}$ \\
\hline
\end{tabular}


0.4072

675.53

0.4114 693.84

0.4156

0.4197

0.4239

0.4281

$1: 5376$

0.3926

0.3967

1. 5501

1.5532

1.5563

1.5593

718.39

$0.4008 \quad 0.4050$

$687.71 \quad 693.82$

0.4091

699.95

0.4132

706.08

0.4173

712.23

0.4214

1.5368

1.5400

1.5431

1.5463

1.5494

1.5525

1.5555

1.5585

$718 \cdot 38$

$0.3947 \quad 0.3988$

0.4028

$\begin{array}{lll}0.3825 & 0.3865 & 0.3906\end{array}$

669.40
1.5361

675.49

681.59

687.69

693.81

699.93

0.4069

1.5486

1.5517

706.07
1.5548

0.4109

712.22

1.5615

0.3767
669.38

0.3807

0.3847

0.3887

1.5353

1.5385

681.57

687.68

0.3927

693.79

0.3968

699.92

0.4008

1.5479

1.5510

706.06

0.4150

718.37

1.5608

$\begin{array}{lll}0.3711 & 0.3751 & 0.3790\end{array}$

669.36

675.45

681.55

0.3830

i. 5346

1.5378

1.5409

0.3869

693.78

0.3909

699.91

0.3948

1.5440

1.5472

1.5502

706.05

712.21

0.4087
718.36

i.5570 1.5600

$\begin{array}{ll}0.3657 & 0.3696\end{array}$

0.3735

0.3774

0.3813

0.3852

699.90

1.5533

0.3987

0.4027

712.20

718035

0.3890

1.5563

1.5593

1.5338

1.5402

(1.0.65

693.77

1.5495

706.04

0.3929

0.3968

1.5464

1. 5526

712019

718.34

$0.3643 \quad 0.3681$

0.3720

0.3758

0.3796

0.3835

1.5556

1. 5586

1340

669.32

675.42

681.52

687.63

693.75

699.88

706.02

0.3873

0.3911

1.5426

1.5457

1.5519

712.18

0.3553

0.3591

0.3629

669.30

675.40

681.50

0.3667

0.3705

0.3743

687.61

693.74

699.87

0.3780

706.01

0.3818

1. 5579

\subsection{4}

0.3541

0.3578

669.28

675.38

0.3616

1.5317

1.5349

681.48

687.60

0.3653

693.72

1.5481

712.16

0.3856

7 i 8.33

1.5572

0.3455

0.3492

0.3690

699.86

0.3728

1.5542

0.3802

1. 5412

1.5443

1.5474

1.550

712.15

718.32

0.3566

0.3603

0.3640

0.3676

1. 5535

1. 5565

1.5311

675.36

681.47

687.58

693.71

699.84

705.99

0.3713

0.3750

1.5405

1.5436

1.5467

I. 5498

712014

718.31

0.3341

0.3376

0.3412

0.3448

0.3483

0.3519

0.3554

105528

1.5558

1.5294

1.5326

1.5357

687.54

693.67

699.81

705.96

0.3590

0.3625

$1.5420 \quad 1.5451$

1.5481

1.5511

718.28

1.5542 


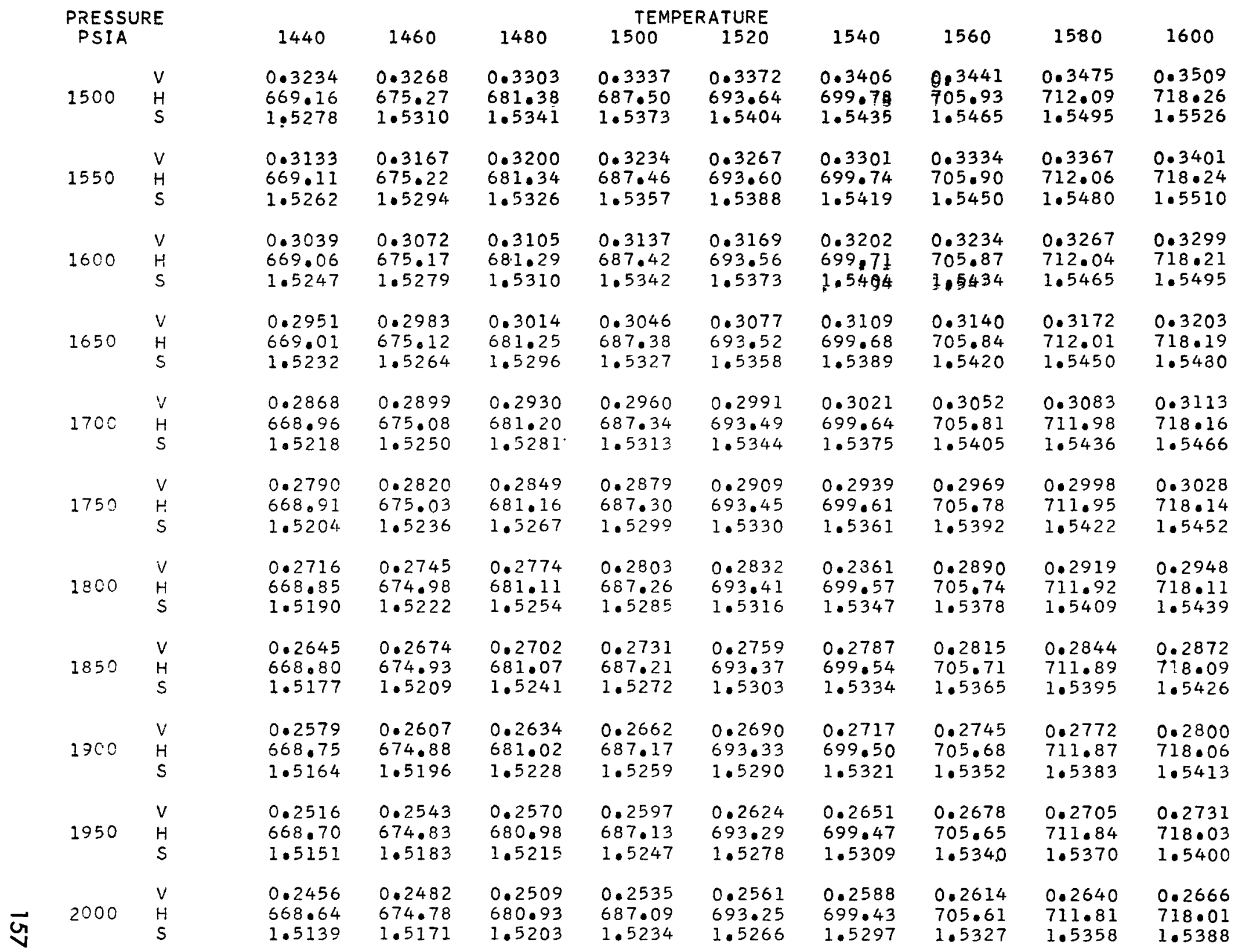

